
UNIVERSIDADE DE BRASÍLIA

FACULDADE DE TECNOLOGIA

DEPARTAMENTO DE ENGENHARIA ELÉTRICA

ANÁLISE DE VULNERABILIDADE DE REDES DE COMUNICAÇÕES POR MEIO DE MÉTRICAS ESTRUTURAIS E ESPECTRAIS DE CONECTIVIDADE

ANTÔNIO CELSO GUIMARÃES TEIXEIRA

ORIENTADOR: WILLIAM FERREIRA GIOZZA

CO-ORIENTADOR: ANDERSON CLAYTON ALVES NASCIMENTO

DISSERTAÇÃO DE MESTRADO EM ENGENHARIA ELÉTRICA ÁREA DE CONCENTRAÇÃO: TELECOMUNICAÇÕES E REDES DE COMUNICAÇÃO DE DADOS

LINHA DE PESQUISA: SEGURANÇA DA INFORMAÇÃO

PUBLICAÇÃO: PPGEE.DM-599/2015

BRASÍLIA/DF: AGOSTO - 2015 
UNIVERSIDADE DE BRASÍLIA

FACULDADE DE TECNOLOGIA

DEPARTAMENTO DE ENGENHARIA ELÉTRICA

\section{ANÁLISE DE VULNERABILIDADE DE REDES DE \\ COMUNICAÇÕES POR MEIO DE MÉTRICAS ESTRUTURAIS E ESPECTRAIS DE CONECTIVIDADE}

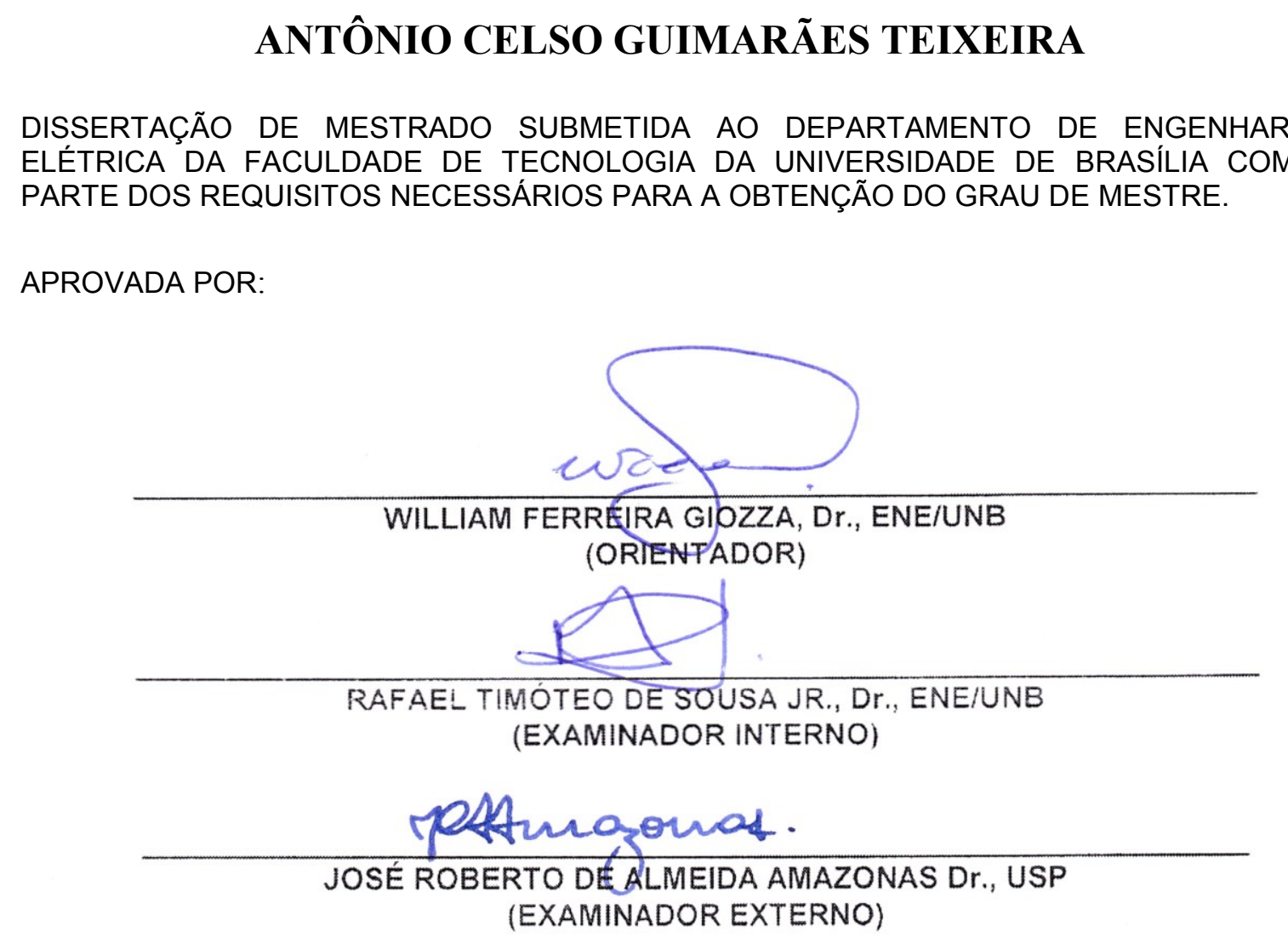




\section{FICHA CATALOGRÁFICA}

GUIMARÃES, ANTÔNIO CELSO TEIXEIRA

Análise de Vulnerabilidade de Redes de Comunicações por meio de Métricas Estruturais e Espectrais de Conectividade [Distrito Federal] 2015.

xxiv, 127p., 210 x 297 mm (ENE/FT/UnB, Mestre, Engenharia Elétrica 2015). Dissertação de Mestrado - Universidade de Brasília. Faculdade de Tecnologia.

Departamento de Engenharia Elétrica

1.Segurança de redes

3.Vulnerabilidades

I. ENE/FT/UnB
2.Infraestruturas Críticas

4.Métricas estruturais e espectrais

II. Título (série)

\section{REFERÊNCIA BIBLIOGRÁFICA}

GUIMARÃES, A. C. T. (2015). Análise de Vulnerabilidade de Redes de Comunicações por meio de Métricas Estruturais e Espectrais de Conectividade. Dissertação de Mestrado em Segurança de Redes, Publicação PPGEE.DM-599/2015, Departamento de Engenharia Elétrica, Universidade de Brasília, Brasília, DF, 127p.

\section{CESSÃO DE DIREITOS}

AUTOR: Antônio Celso Guimarães Teixeira.

TÍTULO: Análise de Vulnerabilidade de Redes de Comunicações por meio de Métricas Estruturais e Espectrais de Conectividade

GRAU: $\quad$ Mestre

ANO: 2015

É concedida à Universidade de Brasília permissão para reproduzir cópias desta dissertação de mestrado e para emprestar ou vender tais cópias somente para propósitos acadêmicos e científicos. $\mathrm{O}$ autor reserva outros direitos de publicação e nenhuma parte dessa dissertação de mestrado pode ser reproduzida sem autorização por escrito do autor.

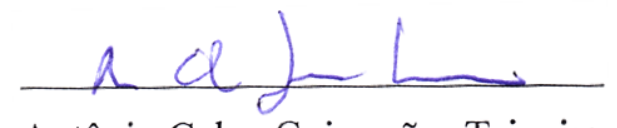

Antônio Celso Guimarães Teixeira

Universidade de Brasília - Faculdade de Tecnologia- Departamento de Engenharia Elétrica CEP 70.910-900 Brasília - DF - Brasil. 


\section{DEDICATÓRIA}

À minha esposa, Vivianne, por seu amor e apoio incondicionais, bem como à minha filha Lisa, que incentivou-me a prosseguir com os estudos. 


\section{AGRADECIMENTOS}

A Deus, acima de tudo e de todos.

Ao Prof. Dr. William Ferreira Giozza, por sua preciosa orientação nas fases intermediárias e finais, com especial destaque para suas contribuições para a completude e aperfeiçoamento deste trabalho, através de múltiplas revisões, cobrindo aspectos de conteúdo, de forma e de metodologia, sem as quais o resultado final aqui obtido nunca teria sido alcançado.

Ao Prof. Dr. Anderson Clayton Alves Nascimento, hoje na Universidade de Washington, em Tacoma, EUA, por sua valiosa orientação nas fases iniciais, desde a escolha do tema até que tomasse uma conformação definitiva, além do seu dedicado acompanhamento e frutuosa colaboração durante as demais fases de desenvolvimento até a conclusão deste trabalho.

À Anatel, que propiciou-me todo o tempo livre necessário e todo seu apoio a esta empreitada, em particular a Jeferson Fued Nacif, a Carlos Manoel Baigorri e a Nelson Mitsuo Takanayagi, que muito me incentivaram a empreender estudos avançados na área de segurança das redes de telecomunicações.

Ao meu dileto colega de trabalho e de mestrado Ricardo de Souza Cerqueira, pelas enriquecedoras trocas de ideias e inestimável colaboração em cuidadosas revisões, que em muito contribuíram à solidez e à qualidade dos resultados finais alcançados.

À União Internacional das Telecomunicações (UIT), em particular ao Prof. Dr. Heung Youl Youm e aos colegas da Comissão de Estudo 17, pelo rico aprendizado e oportunidade de acesso aos mais recentes desenvolvimentos na área de segurança da informação neste ambiente aberto de discussão, frequentado por especialistas internacionais de renome.

À Universidade de Brasília, em particular aos professores e colegas de classe do Programa de Pós Graduação em Engenharia Elétrica, pela oportunidade de cursar este mestrado acadêmico, pelos ensinamentos recebidos, pelas interessantes atividades em grupo e trocas de experiências possibilitadas. 


\title{
RESUMO
}

ANÁLISE DE VULNERABILIDADE DE REDES DE COMUNICAÇÕES POR MEIO DE MÉTRICAS ESTRUTURAIS E ESPECTRAIS DE CONECTIVIDADE

\author{
Autor: Antônio Celso Guimarães Teixeira \\ Orientador: William Ferreira Giozza, PhD \\ Co-Orientador: Anderson Clayton Alves Nascimento, PhD \\ Programa de Pós Graduação em Engenharia Elétrica \\ Brasília, agosto de 2015
}

As redes de telecomunicações são muito resistentes a falhas distribuídas aleatoriamente na topologia, mas são altamente vulneráveis a falhas correlacionadas com a geografia ou a ataques dirigidos a seus pontos críticos. $\mathrm{O}$ impacto da perda de certas estações ou enlaces pode ter um efeito em cascata, extensivo a toda essa infraestrutura crítica.

Este trabalho investiga meios de estimar a capacidade de sobrevivência das redes a tais impactos, em termos de conectividade. Para se obter medições apropriadas, as redes são representadas em forma de grafos, sobre os quais pode-se aplicar métricas estruturais e espectrais. Essas métricas são baseadas em invariantes subjacentes à topologia das redes, modelando sua operação.

Uma composição dessas métricas é aplicada a modelos de redes brasileiras, de maneira a identificar seus nós e enlaces mais críticos. Em seguida, a mesma composição é aplicada para comparar diferentes redes brasileiras e outras redes de dimensões continentais entre si, de forma a classificá-las por ordem de sua vulnerabilidade esperada. Como resultados não esperados, constata-se que, nem os pontos críticos, nem o ranking de vulnerabilidade das redes, são os mais óbvios.

A nosso conhecimento, este é o primeiro estudo que, com base em métricas estruturais e espectrais de grafos, quantifica a vulnerabilidade das redes de telecomunicações brasileiras, tratadas como backbones autônomos.

Palavras chave: segurança de redes, infraestruturas críticas, vulnerabilidade e capacidade de sobrevivência, métricas estruturais e espectrais. 


\begin{abstract}

\author{
Author: Antônio Celso Guimarães Teixeira \\ Supervisor: William Ferreira Giozza, PhD \\ Co-supervisor: Anderson Clayton Alves Nascimento, PhD \\ Electrical Engineering Post-graduate Program \\ Brasília, August 2015
}

ANALYSIS OF VULNERABILITY OF COMMUNICATION NETWORKS BY MEANS OF STRUCTURAL AND SPECTRAL CONNECTIVITY METRICS

Telecommunication networks are very resistant to faults randomly distributed in topology,, but they are highly vulnerable to geographic correlated failures or targeted attacks to their critical points. The impacts of the loss of certain stations or links may have a cascading effect extended over this entire critical infrastructure.

This work investigates ways to estimate the survivability of networks to such impacts, in terms of connectivity. In order to get appropriate measurements, the networks are represented in form of graphs, on which we can implement structural and spectral metrics. Such metrics are based on invariants underlying the topology of networks, modelling its operation.

A composition of these metrics is applied to models of Brazilian networks, in order to identify the most critical nodes and links. Thereafter, the same composition is applied to compare different Brazilian networks and other continental networks together, in order to rank them according to their expected resilience. As unexpected results, we note that, neither critical points, nor the ranking of vulnerability of networks, are the most obvious.

To our knowledge, this is the first study that, based on structural and spectral metrics using graphs, quantifies the vulnerability of Brazilian telecommunications networks, treated as autonomous backbones.

Key words: network security, critical infrastructure, vulnerability and survivability, structural and spectral metrics. 


\section{SUMÁRIO}

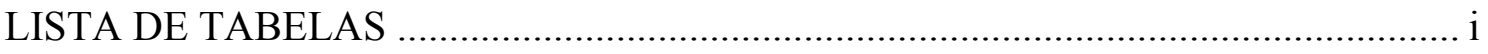

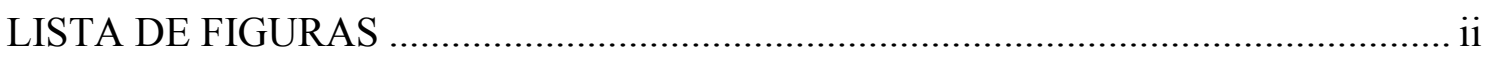

LISTA DE SÍMBOLOS E NOMENCLATURA ……………...................................... iii

LISTA DE SIGLAS E ABREVIAÇÕES …………………………………………..... vi

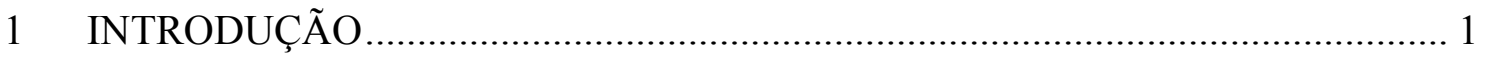

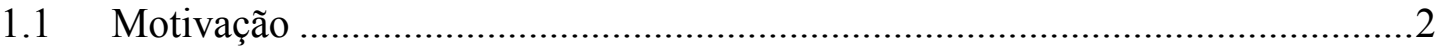

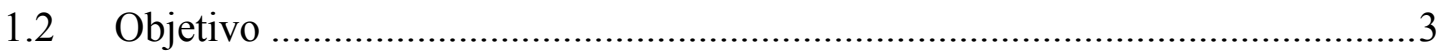

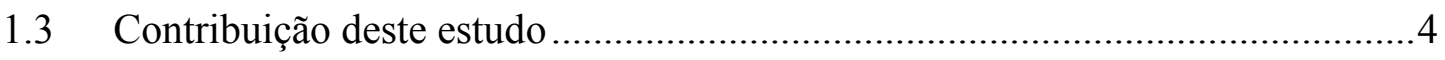

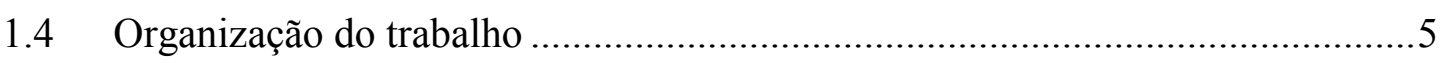

2 VULNERABILIDADE E REDES COMPLEXAS ................................................ 7

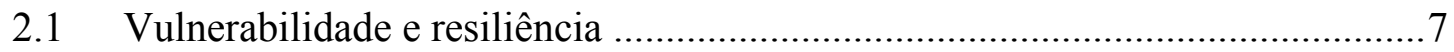

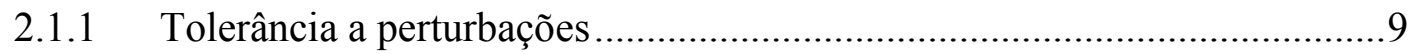

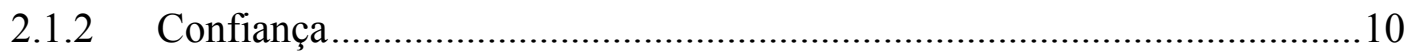

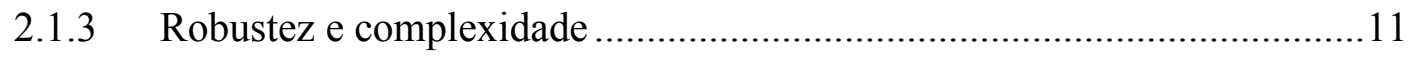

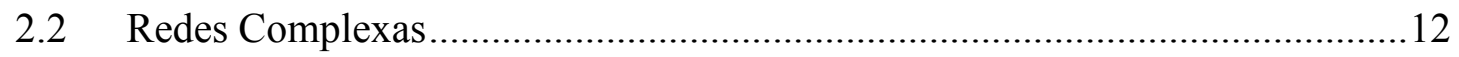

2.2.1 Matrizes de adjacência, laplaciana e normal .............................................13

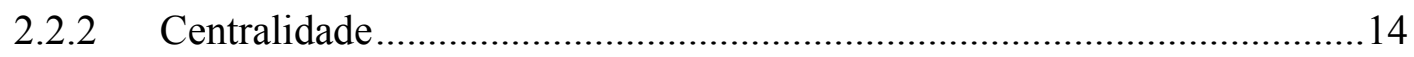

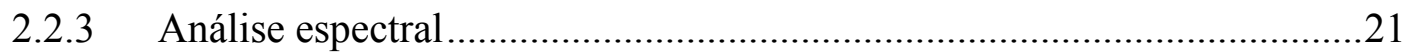

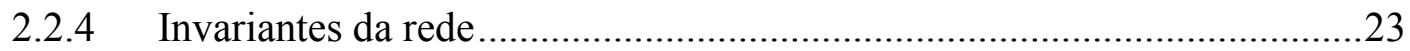

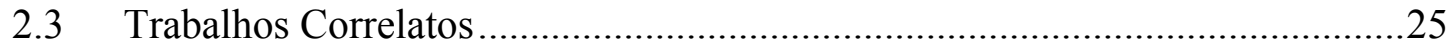

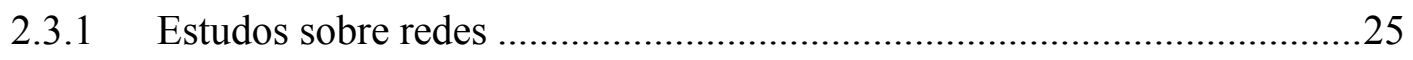

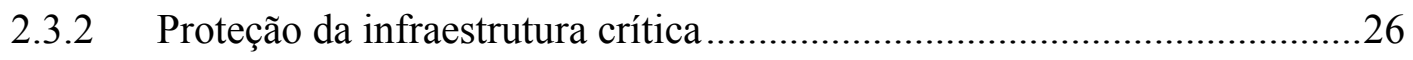

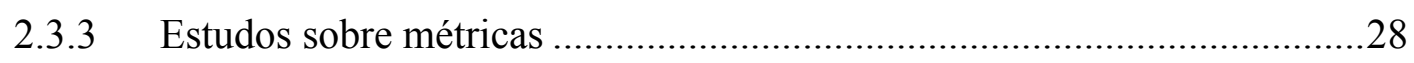

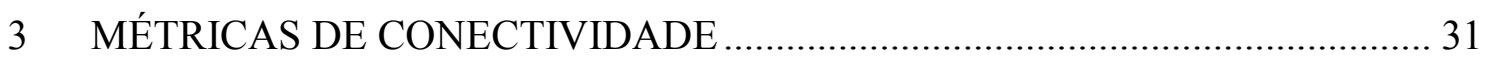

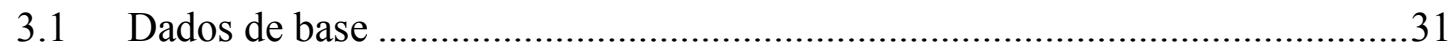

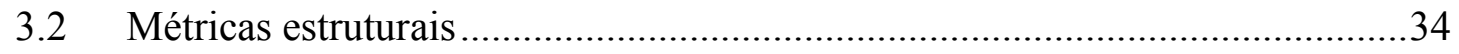

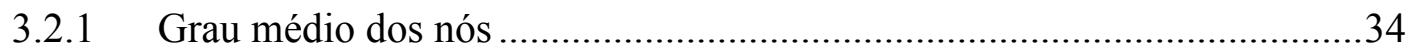

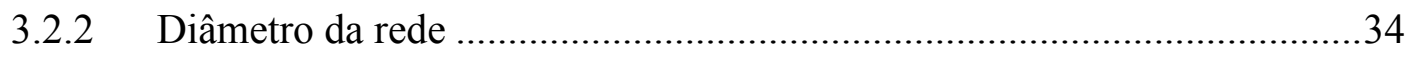

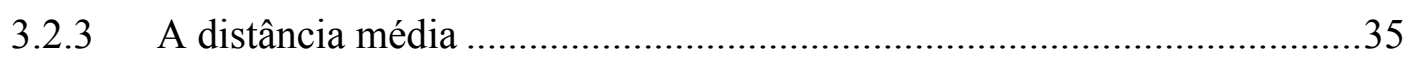

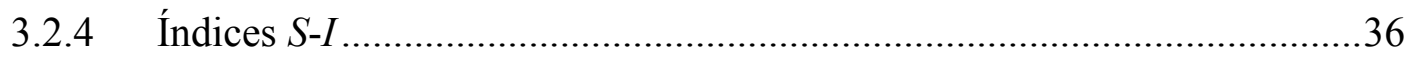

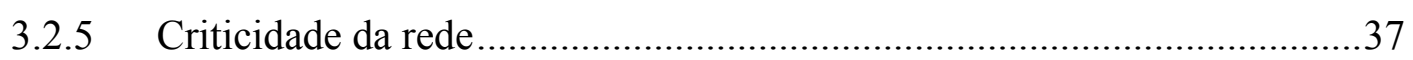

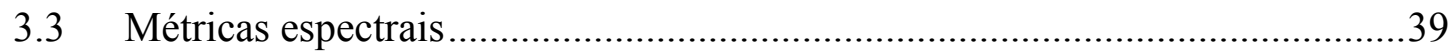

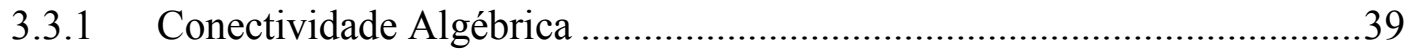

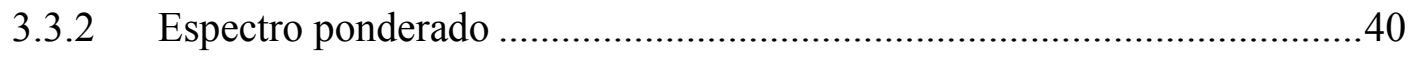

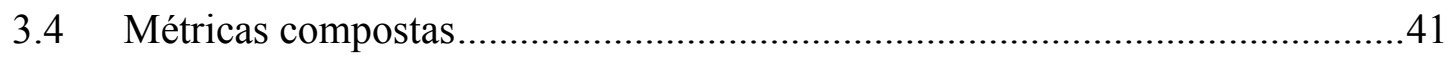




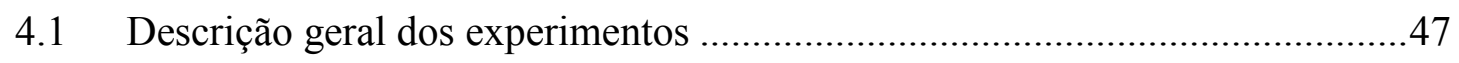

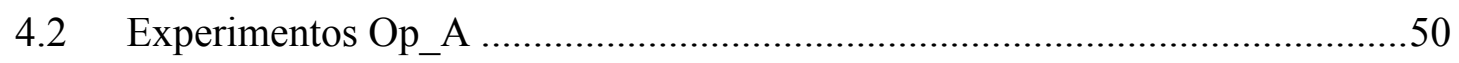

4.2.1 Dados básicos da rede Op_A.............................................................50

4.2.2 Retirada de nós e enlaces da rede Op_A .................................................51

4.2.3 Métrica composta para a rede Op_A ........................................................54

4.2.4 Nós e enlaces mais críticos da rede Op_A ................................................58

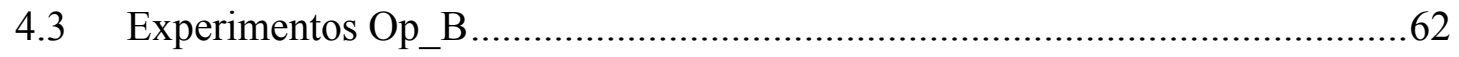

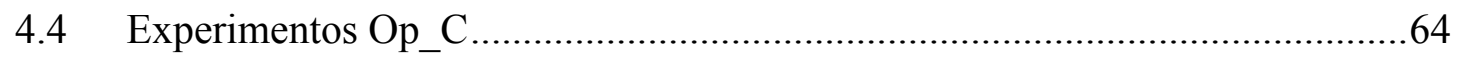

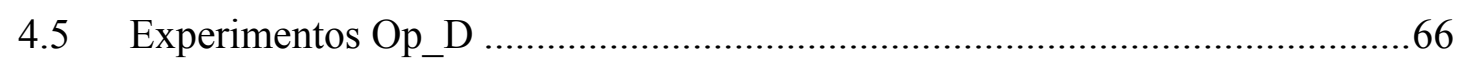

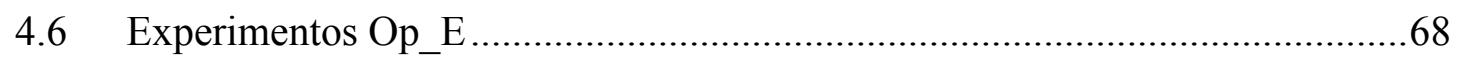

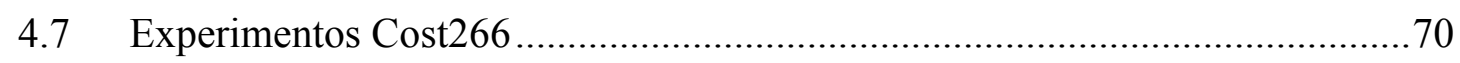

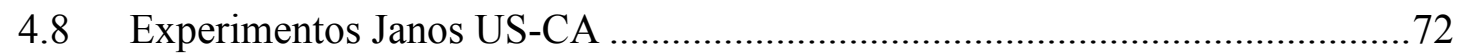

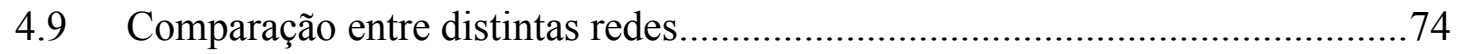

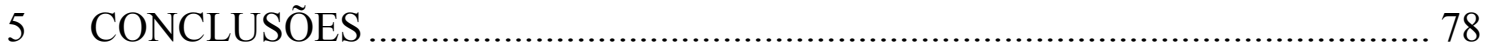

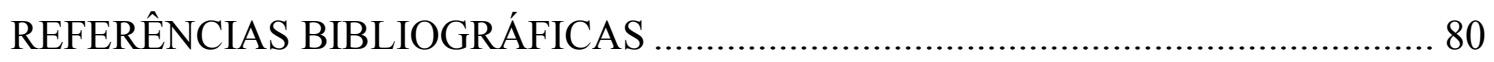

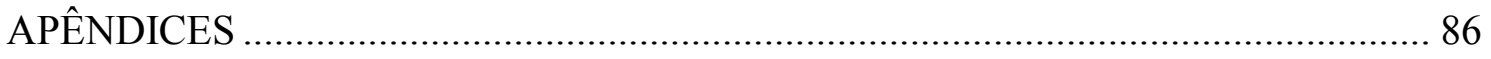

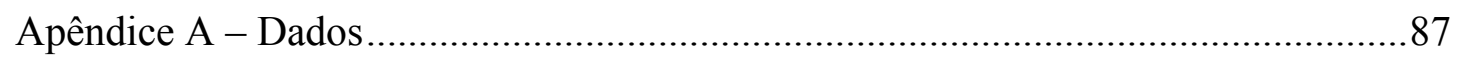

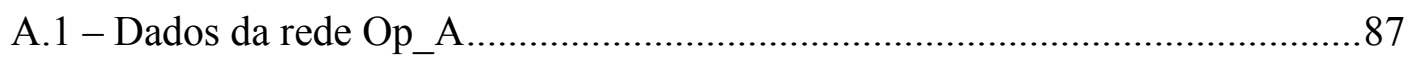

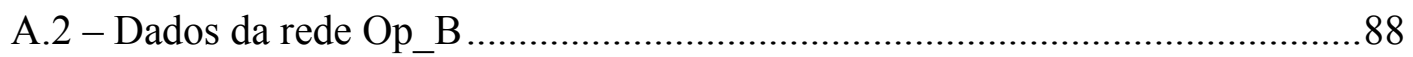

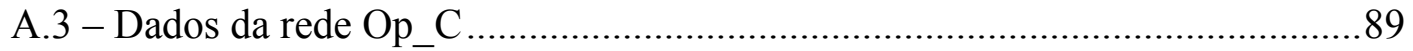

A.4 - Dados da rede Op_D......................................................................... 90

A.5 - Dados da rede Op_E .............................................................................. 91

A.6 - Dados da rede Cost266 ...............................................................................92

A. 7 - Dados da rede Janos US-CA .................................................................93

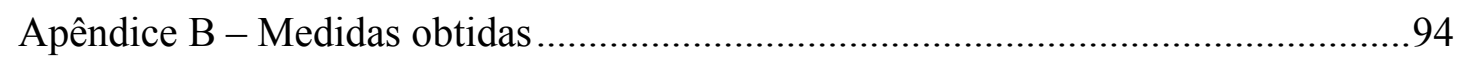

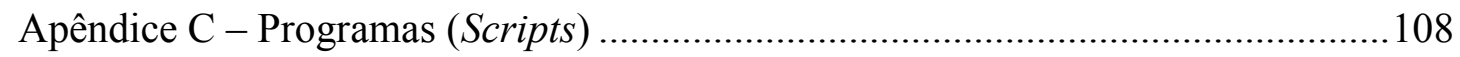

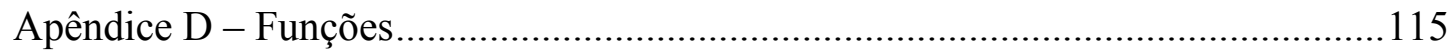

Apêndice E - Estrutura das planilhas utilizadas.....................................................127 


\section{LISTA DE TABELAS}

Tabela 3.1 - Nós do grafo da Figura 3.1, utilizado como exemplo.................................... 32

Tabela 3.2 - Enlaces no grafo da Figura 3.1, utilizado como exemplo.............................. 32

Tabela 3.3 - Representação da matriz de adjacência ........................................................ 32

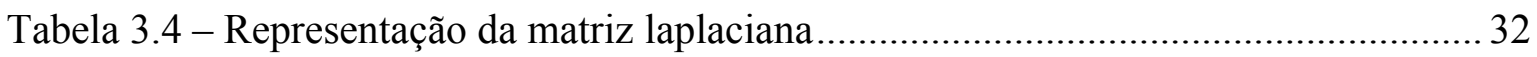

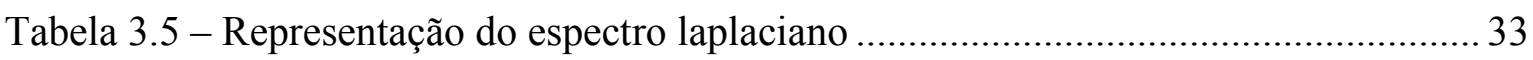

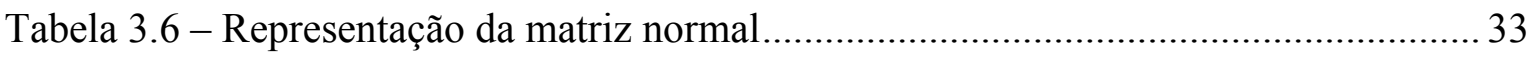

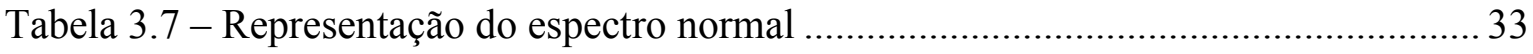

Tabela 3.8 - Representação da matriz de distâncias mínimas........................................... 35

Tabela 3.9 - Distribuição da frequência do número de saltos nos caminhos mais curtos... 37

Tabela 3.10 - Pseudo-inversa de Moore-Penrose da matriz laplaciana ............................. 38

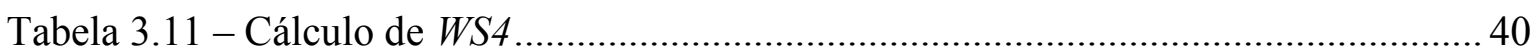

Tabela 3.12 - Valores das métricas com a retirada de enlaces......................................... 43

Tabela 3.13 - Atribuição de pesos pelo método swing weighting...................................... 44

Tabela 3.14 - Variações das medidas com a retirada de enlaces ......................................... 44

Tabela 3.15 - Variações das medidas com a retirada de enlaces $(0$ a 100\%) ..................... 45

Tabela 3.16 - Funções de utilidade individuais e obtenção da métrica composta $C$.......... 45

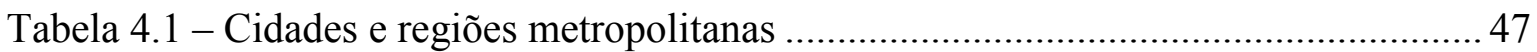

Tabela 4.2 - Dados de entrada da rede Op_A (Nós) ............................................................ 50

Tabela 4.3 - Dados de entrada da rede Op_A (Enlaces) .................................................... 50

Tabela 4.4 - Medidas obtidas para a rede Op_A............................................................. 51

Tabela 4.5 - Valores das medidas para a rede Op_A (Retirando-se nós) ........................... 51

Tabela 4.6 - Valores das medidas para a rede Op_A (Retirando-se enlaces) ...................... 53

Tabela 4.7 - Métrica composta $C$ para a retirada de nós da rede Op_A ............................. 55

Tabela 4.8 - Métrica composta $C$ para a retirada de enlaces da rede Op_A ....................... 57

Tabela 4.9 - Medidas individuais para as redes completas ............................................... 74

Tabela 4.10 - Medidas individuais para as redes com a remoção do nó mais crítico .......... 75

Tabela 4.11 - Medidas individuais para as redes com a remoção do enlace mais crítico... 75

Tabela 4.12 - Medidas individuais para as redes com a remoção do nó mais crítico ......... 75

Tabela 4.13 - Medidas individuais para as redes com a remoção do enlace mais crítico... 75

Tabela 4.14 - Medidas individuais para as redes com a remoção do nó mais crítico ......... 76 


\section{LISTA DE FIGURAS}

Figura 2.1 - Disciplinas da resiliência (adaptado de [12]) .............................................. 8

Figura 2.2 - Interpretação gráfica de um autovalor e autovetor....................................... 23

Figura 3.1 - Exemplo usado para ilustrar o cálculo das métricas ...................................... 31

Figura 3.2 - Grafo resultante da Figura 3.1 com a da retirada do enlace CD .................... 43

Figura 3.3 - Representação gráfica das métricas individuais e da métrica composta $C$..... 46

Figura 4.1 - Variações individuais e métrica $C$ na retirada de nós da rede Op_A.............. 56

Figura 4.2 - Variações individuais e métrica $C$ na retirada de enlaces da rede Op_A........58

Figura 4.3 - Nós e enlaces mais críticos da rede Op_A …..................................................59

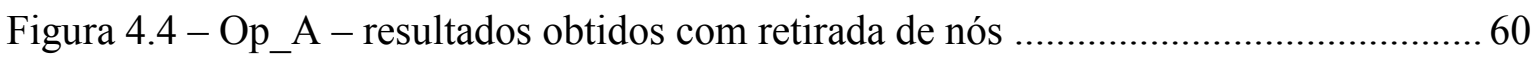

Figura 4.5 -Op_A - resultados obtidos com retirada de enlaces ..................................... 61

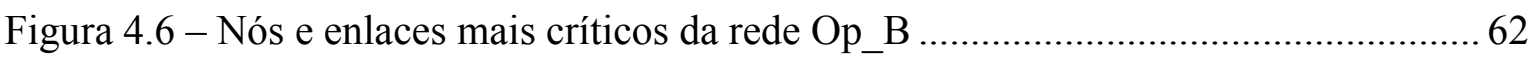

Figura 4.7 - Op_B - resultados obtidos com retirada de nós.............................................. 63

Figura 4.8 -Op_B - resultados obtidos com retirada de enlaces ....................................... 63

Figura 4.9 - Nós e enlaces mais críticos da rede Op_C ........................................................ 64

Figura 4.10 -Op_C - Resultados obtidos com retirada de nós ......................................... 65

Figura $4.11-$ Op_C - Resultados obtidos com retirada de enlaces .................................. 65

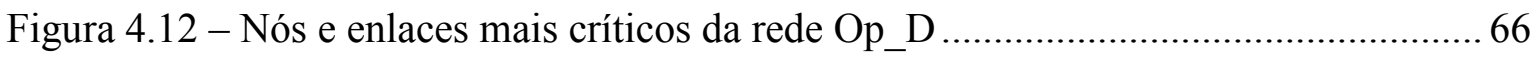

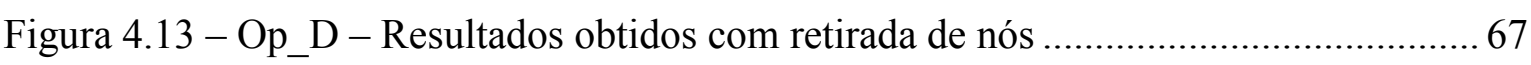

Figura 4.14 - Op_D - Resultados obtidos com retirada de enlaces ................................... 67

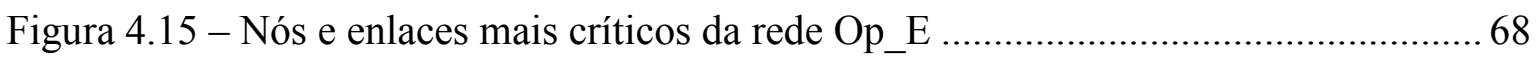

Figura 4.16 - Op_E - Resultados obtidos com retirada de nós........................................... 69

Figura 4.17 -Op_E - Resultados obtidos com retirada de enlaces ................................... 69

Figura 4.18 - Nós e enlaces mais críticos da rede Cost266 ............................................. 70

Figura 4.19 - Cost266 - Resultados obtidos com retirada de nós...................................... 71

Figura 4.20 - Cost266 - Resultados obtidos com retirada de enlaces ............................... 71

Figura 4.21 - Nós e enlaces mais críticos da rede Janos US-CA....................................... 72

Figura 4.22 - Janos US-CA - Resultados obtidos com retirada de nós .............................. 73

Figura 4.23 - Janos US-CA - Resultados obtidos com retirada de enlaces ........................ 73

Figura 4.24 - Impactos da perda do nó ou do enlace mais crítico ...................................... 76

Figura 4.25 - Comparação visual entre todas as redes ..................................................... 77 


\section{LISTA DE SÍMBOLOS E NOMENCLATURA}

$\overrightarrow{1}_{n-1}-$ vetor coluna, com dimensão $(n-1) \times 1$, contendo apenas $1 \mathrm{~s}$

$\boldsymbol{A}$ ou $\boldsymbol{A}(G)$ - matriz de adjacência de um grafo

$A C$ - métrica "conectividade algébrica” (Algebraic Connectivity)

$A D$ - métrica "grau médio dos nós" (Average Degree)

$A S P$ - métrica "distância média" (Average Shortest Path)

$\boldsymbol{B}_{\boldsymbol{d}}$ - matriz de intermediação de percursos aleatórios até o nó $d$ passando por $k$

$\boldsymbol{B}_{\boldsymbol{d}}(d \mid d)$ - matriz $\boldsymbol{B}_{\boldsymbol{d}}$ truncada, removendo a linha $d$ e a coluna $d$

$b_{k}$ - intermediação de percurso aleatório total para o nó $k$

$b_{s k d}$ - intermediação de um percurso aleatório entre $s$ e $d$ para o nó $k$

$\mathrm{c}_{\mathrm{i}}-$ coeficientes do polinômio característico de uma matriz genérica $\boldsymbol{M}$

$d$-designa o nó de destino, em percurso aleatório entre $s$ (origem) e $d$ (destino)

$\boldsymbol{D}$ ou $\boldsymbol{D}(G)$ - matriz diagonal com os graus (ou pesos) dos nós de $G$

Det $(\boldsymbol{M})$ - determinante da matriz $\boldsymbol{M}$ genérica

$d_{i}$ ou $d_{i i}$ - grau do nó $i$ de um grafo, que corresponde ao elemento ii da matriz $\boldsymbol{D}(G)$

$D I A$ - métrica "diâmetro da rede", igual a $h_{\max }$ valor máximo de $h_{i j}$

$\boldsymbol{E}$ - conjunto de enlaces ou arestas de um grafo

$\boldsymbol{f}$ - vetor linha com a distribuição de frequência dos caminhos mais curtos de $G$

$G$ ou $G(\boldsymbol{V}, \boldsymbol{E})$ - grafo binário, constituído de nós $(\boldsymbol{V})$ e enlaces $(\boldsymbol{E})$

$G(\boldsymbol{V}, \boldsymbol{E}, \boldsymbol{W})$ - grafo valorado, com vértices $(\boldsymbol{V})$, enlaces $(\boldsymbol{E})$ e pesos $(\boldsymbol{W})$

$\boldsymbol{H}(G)$ - matriz com o número de saltos (hops) dos caminhos mais curtos entre nós de $G$

$h_{i j}$ - elemento de $\boldsymbol{H}(G)$, número de saltos (hops) no caminho mais curto entre os nós $i$ e $j$

$h_{\max }$ - valor máximo de $h_{i j}$ na matriz de distâncias $\boldsymbol{H}(G)$, igual ao diâmetro de $G$

$\boldsymbol{I}$ - matriz identidade

$I$ - métrica $I$, índice de dispersão da distribuição de frequências de caminhos mais curtos

$k$ - nó intermediário específico em um percurso aleatório entre $s$ e $d$

$\boldsymbol{L}$ ou $\boldsymbol{L}(G)$ - matriz laplaciana do grafo $G$

\section{$l^{+}$- elemento da matriz $\boldsymbol{L}^{+}$}

$m$ ou $|\boldsymbol{E}|$ - número de enlaces (ou arestas) no grafo

$\boldsymbol{M}(i \mid j)$ - matriz truncada, resultante da remoção da $i$-ésima linha e da $j$-ésima coluna

$\boldsymbol{M}^{*}$ - conjugado transposto ou transposto Hermitiano da matriz $\boldsymbol{M}$

$\boldsymbol{M}^{+}$- matriz pseudo-inversa de Moore-Penrose (para qualquer matriz $\boldsymbol{M}$ genérica)

$\boldsymbol{M}^{-1}-$ matriz inversa (para qualquer matriz $\boldsymbol{M}$ genérica) 
$\boldsymbol{M}^{\mathrm{T}}$ - matriz transposta (para qualquer matriz $\boldsymbol{M}$ genérica)

$n$ ou $|\boldsymbol{V}|$ - número de nós (ou vértices) no grafo

$N$ ou $N(G)$ - matriz normal ou laplaciana normalizada do grafo $G$

$N C$ - métrica "criticidade da rede" normalizada

$\boldsymbol{P}_{\boldsymbol{d}}$ - matriz de todas as probabilidades $p_{\text {skd }}$ para o destino $d$

$\boldsymbol{P}_{\boldsymbol{d}}(d \mid d)$ - matriz $\boldsymbol{P}_{\boldsymbol{d}}$ truncada, removendo-se a linha $d$ e a coluna $d$

$\boldsymbol{P}_{d q}$ - matriz de probabilidades de se entrar no nó $k$ no $q$-ésimo salto, entre $s$ e $d$

$p_{i}-$ peso associado à função de utilidade individual $i$

$p_{\text {skd }}$ - probabilidade de um percurso aleatório entre $s$ e $d$ passar pelo nó $k$

$\boldsymbol{Q}$ - matriz auxiliar, utilizada na formula recursiva para obter $\boldsymbol{M}^{+}$a partir de $\boldsymbol{M}$

$q_{i j}$ - elemento da matriz auxiliar $\boldsymbol{Q}$, linha $i$, coluna $j$

$S$ - métrica $S$, índice de simetria da distribuição de frequências de caminhos mais curtos

$s$ - nó de origem genérico (source)

$T(\boldsymbol{u})$ - transformação linear que relaciona autovetores e autovalores $(\mathrm{T}(\boldsymbol{u})=\lambda \boldsymbol{u})$

$\operatorname{Tr}(\boldsymbol{M})$ - traço da matriz $\boldsymbol{M}$ (soma dos elementos de sua diagonal principal)

u ou $u_{i}$-autovetor (se $\boldsymbol{u} \geq 0$ ) de uma matriz

$U_{i}\left(x_{i}\right)$ - função de utilidade individual $i$ para o atributo $x_{i}$

$\boldsymbol{V}$ - conjunto de vértices ou nós de um grafo

$\boldsymbol{W}$ ou $\boldsymbol{W}(G)$ - matriz de valores (ou pesos) associada ao grafo valorado $G(\boldsymbol{V}, \boldsymbol{E}, \boldsymbol{W})$

$w_{i j}$ - valor (ou peso) associado ao enlace situado entre o nó $i$ e o nó $j$

$W_{k}$ - peso do nó $k$, igual à soma dos pesos de todos enlaces nele incidentes

WS - métrica "espectro ponderado"

WS4 - métrica "espectro ponderado", considerando ciclos de 4 no grafo $G$

$\boldsymbol{z}_{n}$ - vetor linha auxiliar, com dimensão $1 \times n$

$\mathrm{Z}_{n-1}-$ vetor coluna auxiliar, com dimensão $(n-1) \times 1$

$\alpha$ ou $\alpha_{i}$ - autovalor da matriz de adjacência $\boldsymbol{A}$

$\gamma$-obliquidade (skewness)

$\Delta$ - variação no valor de uma medida, com relação a um valor de referência

$\varepsilon$ - expoente utilizado na métrica $W S$

$\zeta_{p}$ - vetor linha com dimensão $1 \times p$ (auxiliar em fórmula recursiva)

$\boldsymbol{\eta}_{\boldsymbol{p}}$ - vetor coluna com dimensão $p \times 1$ (auxiliar em fórmula recursiva)

$\lambda$ ou $\lambda_{i}$ - autovalor da matriz $\boldsymbol{L}$ (também usado para autovalor de matriz $\boldsymbol{M}$ genérica)

$\mu-$ média

$v$ ou $v_{i}$-autovalor da matriz normal $N$ 
$\rho$ - métrica "densidade de enlaces"

$\sigma-$ desvio padrão

$\tau$ - métrica "criticidade da rede" não normalizada

$\Phi(s)$ - conjunto de nós adjacentes a um nó $s$ 


\section{LISTA DE SIGLAS E ABREVIAÇÕES}

AAA - Autenticação, Autorização e Auditoria

Anatel - Agência Nacional de Telecomunicações

Backbone - "espinha dorsal" das redes de transporte de telecomunicações

BGP - Border Gateway Protocol

Cost266 - modelo de rede europeia, disponível na SNDlib

CPqD - Centro de Pesquisa e Desenvolvimento em Telecomunicações

CPM - Critical Path Method

DDoS - Ataque de negação de serviço distribuído (Distributed Denial of Service)

Janos US-CA - modelo de rede norte-americana, disponível na SNDlib

MAUT - Teoria da Utilidade Multicritério (Multiple Attribute Utility Theory)

MTTF - tempo médio até falhar (Mean Time to Failure)

MTTR - tempo médio para reparo (Mean Time to Repair)

OPGW - cabo para-raios com fibra ótica (Optical Ground Wire)

PERT - Program Evaluation and Review Technique

PICT - projeto "Proteção da Infraestrutura Crítica em Telecomunicações" da Anatel

QoS - Qualidade de Serviço (Quality of Service)

QoT - Qualidade de Transmissão (Quality of Transmission)

QoE - Qualidade Experimentada (Quality of Experience)

QoI - Qualidade da Informação (Quality of Information)

QoK - Qualidade do Conhecimento (Quality of Knowledge)

SBrT - Simpósio Brasileiro de Telecomunicações

SCADA - Supervisory Control And Data Acquisition

SIEC - projeto "Segurança de Infraestruturas Críticas de Telecomunicações"da Anatel

SNDlib - biblioteca com redes de referência (Standard Network Design Library)

TICs - Tecnologias de Informação e Comunicação

UIT - União Internacional das Telecomunicações

UIT-T - Bureau de Normalização da União Internacional das Telecomunicações 


\section{INTRODUÇÃO}

A Resolução 199 [1], aprovada na 58 a sessão da Assembleia Geral das Nações Unidas, convida seus Estados Membros a desenvolver estratégias para a redução de riscos às infraestruturas críticas de informação e comunicação, bem como a colaborar na criação de uma cultura global de segurança cibernética. A Resolução 199 tem como pano de fundo a crescente dependência de governos, negócios e indivíduos com relação às Tecnologias de Informação e Comunicação (TICs) na provisão de bens e serviços cada vez mais essenciais à vida moderna. As redes de telecomunicações são uma parte vital destas infraestruturas.

Infraestrutura crítica é o termo utilizado por governos para referir-se a instalações ou organizações vitais para o interesse público, cuja falta ou deficiência pode levar à escassez de oferta, perturbação da ordem pública ou a impactos similares. A expressão "crítica" não se refere apenas à importância da infraestrutura em si, mas sim ao fato de que vulnerabilidades com impactos globais para a sociedade estão concentradas em certos "pontos críticos" específicos. A natureza conectada desses pontos críticos leva à possibilidade de se "agir a distância" e de se gerar "efeitos em cascata" ou "efeitos multiplicativos". A proteção de uma infraestrutura crítica e a busca da redução de suas vulnerabilidades deve estar focada nesses pontos críticos [2].

Uma rede representada por um grafo que apresenta uma estrutura topográfica não trivial costuma ser referida como uma rede complexa. Estudos mostraram que as redes de telecomunicações são redes complexas, que tendem a se auto organizar como redes não planas, formando topologias dos tipos "pequeno mundo" e "livres-de-escala" [3]. Os atributos gerais dessas auto-organizações são a conexão preferencial com os nós mais próximos e a formação de aglomerados locais (clusters) que se interconectam uns aos outros através de conexões globais. Isso conduz a uma estrutura eficiente, mas esparsamente conectada. É uma estrutura resistente a falhas aleatórias, mas muito susceptível a ataques dirigidos a seus nós e enlaces críticos. Estudos feitos no início deste século, por exemplo, indicaram que o corte de $1 \%$ dos roteadores mais críticos da Internet reduziria seu desempenho à metade e que a rede chegaria ao colapso com a perda de apenas $4 \%$ desses roteadores [3].

Esses pontos críticos costumam ser chamados os conectores globais da rede. O que leva às falhas mais catastróficas costuma ser a perda de tais elementos. Assim, os estudos 
de vulnerabilidade costumam focar na identificação de conectores globais, bem como na análise do que ocorre com a rede como um todo, em casos de falhas ou ataques dirigidos a tais pontos críticos. Para isso é necessário dispor de métricas adequadas e aplicá-las a modelos realistas das redes de telecomunicações de interesse.

\subsection{Motivação}

O presente estudo se interessa pela proteção das infraestruturas críticas de telecomunicações, que são compostas de dispositivos centrais, intermediários ou terminais, suportados por uma ou várias redes que os interconectam. Para protegêlas é necessário identificar seus pontos críticos. Ocorre que, devido à sua topologia particular, as redes de telecomunicações são bastante resistentes a falhas distribuídas aleatoriamente na topologia, mas são altamente vulneráveis a falhas relacionadas com a geografia ou a ataques dirigidos a seus pontos críticos. Assim, perturbações de vários tipos envolvendo pontos críticos, tais como desastres naturais, ataques físicos ou cibernéticos, podem ter um impacto considerável [3].

O mapeamento dos pontos críticos de uma rede de telecomunicações costuma ser feito medindo-se o impacto da retirada de cada nó ou enlace da rede. Para tal fim, pode-se utilizar métricas de conectividade existentes, que têm um grande espectro de aplicação [4]. Tais métricas de conectividade de redes costumam ser baseadas na teoria dos grafos, uma parte importante da matemática discreta, que tem um campo de aplicação muito amplo, em engenharia, biologia, sociologia, entre outros [5]. Uma grande variedade de métricas pode ser adequada a avaliar a vulnerabilidade das redes, cada uma delas permitindo capturar diferentes características dos grafos representativos das redes. Essas métricas são aplicáveis em domínios estruturais ou espectrais [6]. Nesse último caso, recorre-se à teoria espectral dos grafos, que tem por objetivo identificar os principais invariantes de uma rede. Pode-se assim medir propriedades relacionadas com a estrutura valendose do espectro do grafo, que são os autovalores de certas matrizes a ele associadas [7]. 


\subsection{Objetivo}

O objetivo do presente estudo é identificar os principais pontos críticos de redes de telecomunicações através da quantificação de seu impacto sobre a rede como um todo. Além disso, também busca comparar distintas redes entre si, confrontando suas vulnerabilidades relativas, expressas em termos de métricas de conectividade.

O termo vulnerabilidade costuma ser conceituado como uma deficiência potencial de um sistema, tal que permite que perturbações externas possam comprometer seu funcionamento normal. No presente estudo busca-se identificar e quantificar a vulnerabilidade das redes em termos de sua capacidade de sobrevivência, expressa através de métricas de conectividade existentes.

Assim, o objetivo deste estudo consiste em responder às seguintes questões, relativas a redes de telecomunicações:

- Quais métricas utilizar para quantificar a vulnerabilidade das redes?

- Como identificar os pontos vulneráveis (conectores globais) de uma rede?

- Como comparar as vulnerabilidades de distintas redes entre si?

A hipótese que se pretende demonstrar no presente trabalho é que a vulnerabilidade das redes de telecomunicações a falhas de caráter geográfico ou a ataques localizados dirigidos a seus nós críticos pode ser estimada através de uma composição adequada de métricas de conectividade de grafos.

A metodologia utilizada para demonstrar esta hipótese consiste em analisar as métricas de conectividade existentes e, a partir delas trabalhar com uma métrica composta, tal que permita capturar adequadamente a influência de cada métrica individual, levando à identificação dos nós e dos enlaces mais críticos de uma rede. Para validar a metodologia, a métrica composta é aplicada a modelos realistas de redes de telecomunicações baseados em grafos, representando redes de operadoras brasileiras mais algumas redes internacionais disponíveis na SNDlib [8], utilizadas como referência.

Os resultados observados nessa avaliação podem ser úteis para elaborar recomendações de política pública ou de planejamento, com vistas a mitigar os efeitos adversos de eventuais ataques dirigidos ou catástrofes naturais. 


\subsection{Contribuição deste estudo}

Faz-se aqui um estudo de caso sobre a vulnerabilidade dos backbones de telecomunicações do Brasil, baseado em métricas de conectividade existentes. Além disso, trabalha-se na combinação de distintas métricas, buscando obter uma métrica multicritérios, tal que permita não apenas identificar nós e enlaces mais críticos, mas que sirva igualmente para comparar as redes brasileiras entre si e com redes de referência internacionais similares.

As redes de telecomunicações do Brasil, em sua maioria pertencem a empresas privadas, que têm atuação nacional ou apenas regional. Essas redes costumam ser operadas de forma integrada, quando pertencem a um mesmo grupo econômico ou de forma autônoma, em caso contrário. Forma-se assim um certo número de backbones independentes, com relativamente poucos pontos de interconexão entre eles. Até onde se tem conhecimento, há uma escassez de estudos sobre as vulnerabilidades das redes brasileiras, em particular as que têm alcance nacional.

Desta forma, este seria o primeiro estudo que busca identificar e quantificar vulnerabilidade das redes brasileiras de cobertura nacional, tratadas como distintos backbones autônomos. O estudo contribui em permitir saber como modelos representativos dessas redes se comportam diante da perda de nós e enlaces. Esses modelos permitem também comparar as distintas redes entre si e com referências internacionais muito utilizadas em estudos similares.

Além disso, o presente estudo também contribui ao propor uma combinação de quatro métricas estruturais e espectrais de conectividade distintas para avaliar a vulnerabilidade das redes. Permite assim fazer uma análise com múltiplos critérios, com recurso a uma única métrica composta, aplicável a redes de telecomunicações de porte e topologias razoavelmente similares aos das redes brasileiras. Para verificar sua validade e aplicabilidade, a métrica composta foi testada em contextos próximos à realidade, em particular em modelos redes de telecomunicações de dimensões continentais bem conhecidas dos Estados Unidos e da Europa [8].

Como resultado deste estudo, um artigo foi submetido e aceito para o XXXIII Simpósio Brasileiro de Telecomunicações, SBrT2015, realizado em Juiz de Fora, MG, de 1 a 4 de setembro de 2015. Este estudo também produziu uma 
contribuição brasileira apresentada à Comissão de Estudos 17 (Segurança) da UIT-T.

\subsection{Organização do trabalho}

Além deste capítulo introdutório, o presente trabalho foi estruturado em quatro outros capítulos, complementados por cinco apêndices que contêm as ferramentas e dados utilizados.

O Capítulo 2 abordada os aspectos teóricos relevantes ao presente trabalho, em particular os relativos às disciplinas da Resiliência e à Teoria Espectral dos Grafos, além de revisitar os principais trabalhos correlatos existentes.

No Capítulo 3 discute-se em detalhes as métricas de conectividade aqui adotadas e apresenta-se uma métrica composta proposta com vistas a identificar conectores globais, quantificar seus impactos e permitir a comparação entre distintas redes de telecomunicações em termos de vulnerabilidade.

O Capítulo 4 apresenta a aplicação dessas métricas de conectividade a modelos de redes baseados em grafos, brasileiros ou internacionais. Os modelos experimentados, as medidas obtidas nas simulações realizadas e uma discussão sobre os resultados individuais obtidos são apresentados nesse capítulo. O Capítulo 4 termina com uma análise global dos resultados, incluindo uma comparação direta entre a vulnerabilidade das redes ensaiadas.

As conclusões deste trabalho e considerações sobre possíveis trabalhos futuros são apresentadas no Capítulo 5.

As tabelas de dados utilizadas como entradas para todas as simulações realizadas estão apresentadas no Apêndice A.

No Apêndice B estão tabuladas todas as medidas individuais obtidas no curso dos experimentos realizados, acompanhadas das respectivas representações gráficas.

No Apêndice C estão as listagens dos programas principais (scripts) desenvolvidos em MATLAB para efetuar as simulações necessárias à obtenção das métricas individuais nos experimentos realizados com cada uma das redes.

No Apêndice D são apresentadas as funções desenvolvidas em MATLAB para efetuar os cálculos requeridos para as simulações aqui realizadas. 
Finalmente, um exemplo que mostra as partes essenciais da planilha MS-Excel utilizada para as entradas, resultados intermediários e saídas das simulações figura no Apêndice E. 


\section{VULNERABILIDADE E REDES COMPLEXAS}

Este capítulo trata de bases teóricos relevantes ao presente trabalho. De início, são passadas em revista as disciplinas da Resiliência e suas interrelações, com ênfase nos conceitos de vulnerabilidade e conectividade. Em seguida, são abordadas as redes complexas com algum aprofundamento na teoria dos Grafos e na Teoria Espectral dos Grafos. Finalmente, são revisitados os principais trabalhos correlatos que versam sobre redes complexas, proteção da infraestrutura crítica de telecomunicações e métricas de conectividade de interesse para este trabalho.

\subsection{Vulnerabilidade e resiliência}

O termo vulnerabilidade costuma ser conceituado como uma deficiência potencial de um sistema tal que permite que perturbações externas possam comprometer seu funcionamento normal. Por outro lado, no caso de redes, a resiliência é entendida como a habilidade de prover e manter um nível aceitável de serviço diante de falhas ou ataques [9].

No presente estudo, o tema de interesse é identificar e quantificar a vulnerabilidade das redes, em termos de sua capacidade de sobrevivência expressa através de métricas de conectividade, que fazem parte das disciplinas da resiliência. Nesta seção, busca-se melhor entender os principais conceitos envolvidos e suas inter-relações.

O estudo da resiliência de redes envolve uma ampla variedade de disciplinas. Como estas disciplinas se desenvolveram de maneira independente, por distintas organizações e ao longo de vários anos, ainda não possuem uma classificação bem estabelecida. Tampouco há terminologias consistentes e universalmente aceitas. Uma vulnerabilidade provém de uma deficiência potencial do sistema, que tanto pode vir de uma falha de projeto acidental (como um bug de software) quanto de uma deficiência intencional. Uma vulnerabilidade pode permitir que um ataque externo resulte em uma interrupção ou qualquer degradação do funcionamento normal caso a concepção do sistema não seja suficientemente robusta para evitá-la, devido a restrições de custo, por exemplo.

Tradicionalmente, tanto os mecanismos de resiliência quanto suas medidas, têm desafios e domínios específicos. A capacidade de sobrevivência costuma ser quantificada em termos de disponibilidade ou de modelos de desempenho da rede. A resiliência pode ser quantificada com uma medida de desempenho transitório ou de disponibilidade da rede quando sujeita a solicitações que ultrapassam seus parâmetros de projeto. Métricas 
orientadas a serviço incluem medidas de perda de capacidade de tráfego durate uma falha e medidas relativas à interrupção total do serviço [10]. Com base na distinção usual entre fabricantes de equipamentos, provedores de serviços e usuários finais, métricas específicas foram desenvolvidas para aplicação em cada domínio. No campo da segurança de redes, uma abordagem usual é a análise de vulnerabilidades, com o fito de determinar como a rede responde a riscos de segurança [11].

Avaliar a resiliência é mais difícil que avaliar redes em termos de métricas tradicionais de segurança. Trata-se de avaliar a capacidade da rede de continuar a prover serviços em nível aceitável, enquanto enfrenta diversos tipos de perturbações. A avaliação da resiliência quantifica a degradação do serviço em presença de perturbações no estado operacional da rede.

Sterbenz et al. [12] propõem organizar as disciplinas relacionadas com a resiliência em duas categorias de base, como se mostra na Figura 2.1.

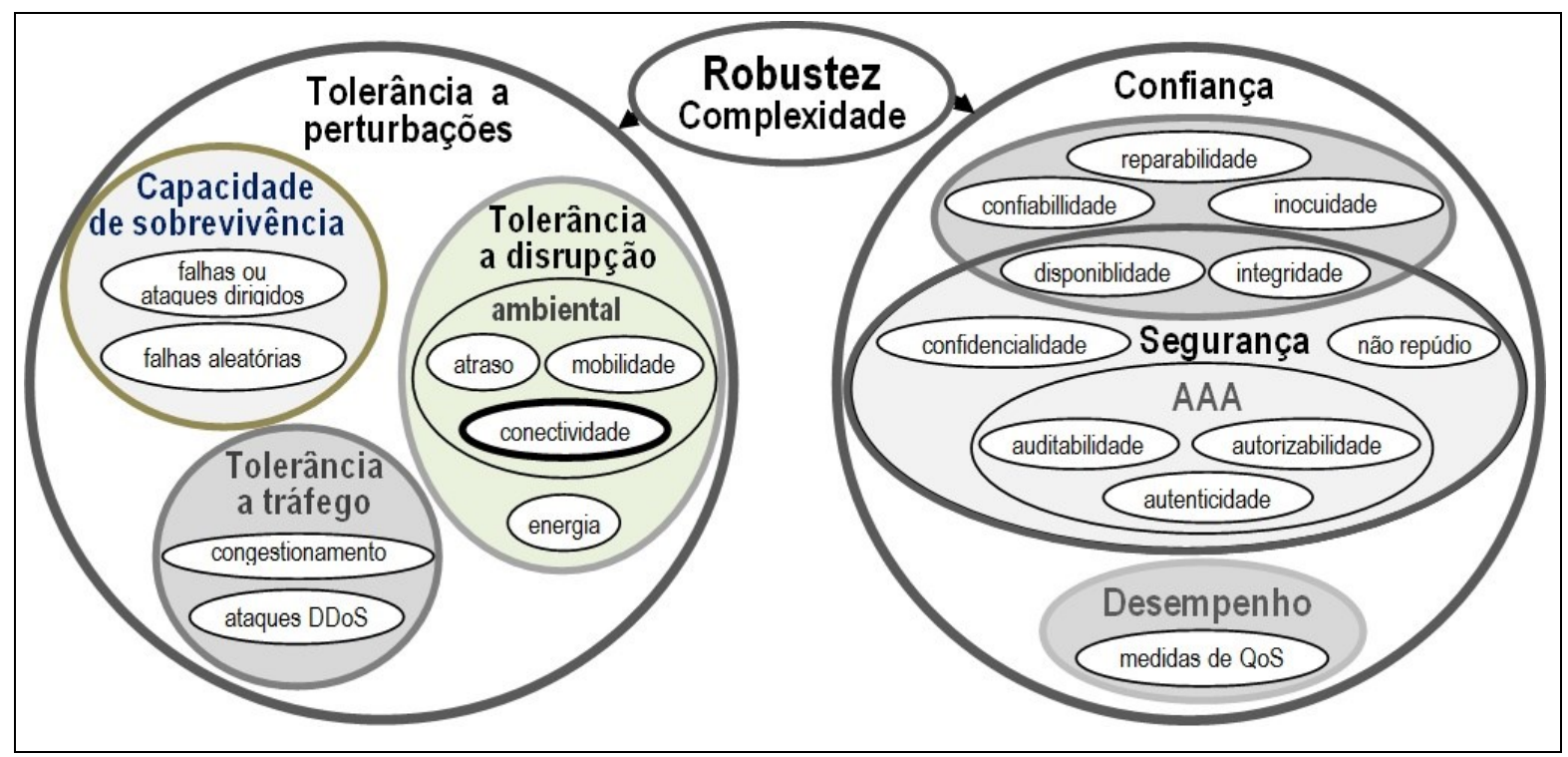

Figura 2.1 - Disciplinas da resiliência (adaptado de [12])

Em nível bem alto, divide-se as disciplinas da resiliência em duas categorias. No lado esquerdo da Figura 2.1 estão as disciplinas relacionadas com a tolerância a falhas, que lidam com o projeto e a engenharia de sistemas. São as que asseguram a continuidade do serviço em casos de falhas. No lado direito estão as disciplinas relacionadas com a confiança, que descrevem propriedades mensuráveis dos sistemas resilientes. A relação entre essas duas classes corresponde aos conceitos de robustez e de complexidade. 


\subsubsection{Tolerância a perturbações}

O primeiro grande subconjunto de disciplinas de resiliência lida com o problema de como projetar sistemas de maneira que sejam tolerantes a perturbações que impeçam ou degradem a prestação de serviço desejada.

Os problemas enfrentados podem ser subdivididos em:

- capacidade de sobrevivência, que trata da tolerância a falhas de componentes ou de subsistemas, aleatórias, naturais ou provocadas pelo homem;

- tolerância à disrupção, que considera faltas de energia elétrica ou perturbações de vias de comunicação por fatores ambientais que causam atrasos, perdas de mobilidade ou de conectividade nas redes; e

- resistência ao tráfego, que inclui congestionamentos, variações drásticas de demanda ou injeções indevidas de tráfego na rede provocadas por ataques distribuídos de negação de serviço (DDoS).

A tolerância a falhas é uma das disciplinas da resiliência estudadas há mais tempo. É definida como a habilidade de um sistema a resistir a perturbações de maneira tal que não resultem em falhas de serviço do sistema. A tolerância a falhas faz uso da redundância como técnica para compensar falhas aleatórias e não correlacionadas de componentes. No entanto, a tolerância a falhas não é suficiente para proteger contra falhas correlacionadas, não sendo assim suficiente para prover resiliência. A tolerância a falhas é considerada como um subconjunto da capacidade de sobrevivência, que inclui falhas múltiplas e correlacionadas. O aparecimento da Internet levou ao desenvolvimento de novas técnicas, aplicáveis a redes não limitadas (unbounded networks), que podem ser afetadas por falhas correlacionadas, de tal forma que as técnicas de tolerância falhas tradicionais não são suficientes.

A capacidade de sobrevivência é definida como a habilidade do sistema de cumprir com sua missão, de maneira tempestiva, na presença de ameaças, tais como ataques em larga escala ou grandes desastres naturais. Além da redundância requerida pela tolerância a falhas, a capacidade de sobrevivência requer diversidade, de maneira que uma mesma ocorrência seja pouco provável de ser comum a distintas partes de um mesmo sistema afetadas por falhas correlacionadas. A capacidade de sobrevivência costuma ser quantificada usando cadeias de Markov multidimensionais [12], nas quais uma dimensão 
captura os componentes das falhas enquanto a outra dimensão mede o desempenho do sistema diante de falhas simultâneas.

Outro importante tipo de desafio, específico às redes de telecomunicações, diz respeito a problemas ambientais de comunicação que tornam difícil manter conexões fima-fim estáveis entre os usuários. A tolerância à disrupção é definida como a capacidade de um sistema de tolerar interrupções na conectividade entre os seus componentes, devidas a causas ambientais, tais como interrupções em um canal, problemas de mobilidade, longos atrasos de forma imprevisível, bem como a tolerância à falta de energia elétrica.

A última grande categoria de desafios é causada pela injeção de tráfego extra na rede. A tolerância a tráfego é entendida como a capacidade de um sistema para absorver uma solicitação de carga imprevisível sem uma redução significativa de desempenho ou um colapso por congestionamento. O tráfego extra aqui referido é aquele que está além dos parâmetros de projeto da rede, previstos para sua operação normal. A injeção de tráfego extra inesperada pode ser legítima, como em casos de demanda pontual (flash crowd) ou maliciosa, como em casos de ataque distribuído de negação de serviço (Distributed Denial of Service - DDoS). É importante notar que os recursos de rede sofrem impacto independentemente de que o tráfego extra seja malicioso ou não. Um ataque DDoS suficientemente sofisticado pode ser indistinguível de tráfego normal. Portanto, os mecanismos de tolerância ao tráfego são importantes, independentemente de que possam haver mecanismos de detecção de ataques de negação de serviço [12].

\subsubsection{Confiança}

A confiança (trustworthness) é definida como a garantia de que um sistema irá se comportar conforme o esperado, no que diz respeito a propriedades mensuráveis. Essas disciplinas consistem em:

- confiança no funcionamento (dependability);

- segurança; e

- desempenho.

Confiança no funcionamento (dependability) é a disciplina que quantifica a certeza que se pode ter de que o serviço esperado será efetivamente entregue pelo sistema. Consiste em dois aspectos principais: disponibilidade e confiabilidade. Importantes para ambos esses aspectos são os valores esperados das funções de densidade de falhas e de reparos. Uma medida básica de funcionamento é o tempo médio até falhar (MTTF), que é 
o valor esperado da função densidade de falhas. Outra medida básica é o tempo médio para reparo MTTR, que é o valor esperado da função densidade de reparos. Adicionalmente, há ainda outros aspectos relacionados à confiança no funcionamento. A reparabilidade é a aptidão do sistema para passar por reparos e evoluções. A inocuidade (safety) é a capacidade do sistema em não provocar problemas ou falhas catastróficos, um aspecto particularmente importante para certas infraestruturas críticas, como a rede elétrica ou plantas nucleares que dependem da Internet e de sistemas SCADA (Supervisory Control And Data Acquisition). A integridade é um aspecto da confiança de funcionamento também associado à segurança, como se descreve a seguir.

A segurança é uma disciplina que trata da proteção de atividades humanas e de medidas tomadas de forma a impedir acessos ou alterações não autorizados. As propriedades de segurança incluem as chamadas AAA (autenticação, autorização, auditoria), confidencialidade e não repúdio. Além disso, a segurança compartilha com a confiabilidade as propriedades de disponibilidade e integridade. No contexto da confiabilidade considera-se as propriedades mensuráveis de aspectos de segurança.

O desempenho é a propriedade de um sistema que representa o nível de qualidade exigido pela especificação do serviço ou perfil de desempenho (QoS, QoT, QoE, QoI ou QoK), tais como medidas de atraso, velocidade ou taxa de entrega de pacotes, etc. As medidas de desempenho costumam quantificar a degradação de um sistema de forma progressiva. São assim uma forma de se estender para o caso contínuo os conceitos binários tratados na disciplina de confiança.

\subsubsection{Robustez e complexidade}

No diagrama da Figura 2.1 aparecem mais duas disciplinas de ligação, que estão fora do que foi tratado nas Seções 2.1.1 e 2.1.2, mas que descrevem a relação entre essas disciplinas e certas características globais de um sistema. São as disciplinas de robustez e de complexidade.

A noção de robustez vem da teoria de controle e corresponde a uma propriedade do sistema de manter seu desempenho, mesmo na presença de perturbações em suas entradas. No contexto da resiliência, robustez corresponde a um atributo similar para um sistema, que quantifica o efeito de perturbações que alteram seu comportamento. 
Complexidade, por outro lado, se refere ao estudo de fenômenos que aparecem quando um grande número de sistemas interagem entre si, resultando em comportamentos novos. A disciplina da complexidade tem uma importante relação com a resiliência, posto que mecanismos destinados a prover resiliência podem levar ao aumento da complexidade e esse aumento de complexidade, por sua vez, pode resultar em maior vulnerabilidade.

\subsection{Redes Complexas}

O estudo de redes complexas remete à teoria dos grafos, que é parte importante da matemática discreta. $\mathrm{O}$ termo redes complexas refere-se a um grafo que apresenta uma estrutura topográfica não trivial.

Um grafo é um diagrama constituído por um conjunto finito de nós (ou vértices) com enlaces (ou arestas) que os interligam. Os grafos têm ampla aplicação no estudo de redes em geral, tais como malhas rodoviárias, rotas aéreas ou redes de telecomunicações. Além disso, são muito utilizados em diversos outros domínios, como em pesquisa operacional, PERT/CPM, análise de circuitos elétricos, estudo de ecossistemas biológicos, química orgânica, sociologia e economia, entre outros [13].

Uma rede de telecomunicações é um conjunto de nós $\boldsymbol{V}$ (ou vértices) conectados por um conjunto de enlaces $\boldsymbol{E}$ (ou arestas) que pode ser representada por um grafo $G(\boldsymbol{V}, \boldsymbol{E})$. No que se segue, considera-se apenas grafos que não possuem enlaces ligando um nó a ele mesmo (isto é, sem loops ou laços), sem enlaces múltiplos (isto é, não há mais de um enlace incidindo no mesmo par de vértices) e sem orientação. Estes grafos são chamados grafos simples, mas serão aqui referidos simplesmente como grafos. Os enlaces podem ter valores (ou pesos) associados. Esses valores podem representar um atributo associado ao enlace, como capacidade, disponibilidade, custo ou algum outro qualquer outro parâmetro que se queira representar. Nesse caso, os grafos são ditos grafos valorados ou grafos ponderados. Se esses valores podem ser apenas 0 ou 1, o grafo é dito binário.

Definindo mais formalmente, um grafo binário é uma estrutura $G=G(\boldsymbol{V}, \boldsymbol{E})$, constituída por um conjunto finito e não vazio $\boldsymbol{V}$, cujos elementos são denominados nós (ou vértices), e um conjunto $\boldsymbol{E}$, constituído de subconjuntos a dois elementos de $\boldsymbol{V}$, denominados enlaces (ou arestas).

Um grafo valorado é uma estrutura $G=G(\boldsymbol{V}, \boldsymbol{E}, \boldsymbol{W})$, constituída por um conjunto finito e não vazio $\boldsymbol{V}$, com elementos denominados nós (ou vértices), um conjunto $\boldsymbol{E}$, 
constituído de subconjuntos a dois elementos de $\boldsymbol{V}$, denominados enlaces (ou arestas) e um conjunto de valores (ou pesos) $\boldsymbol{W}$ associados a seus enlaces $\boldsymbol{V}$.

Indica-se por $n=|\boldsymbol{V}|$ e $m=|\boldsymbol{E}|$, respectivamente, o número de vértices e o número de arestas de $G$. Se $i, j \in \boldsymbol{V}$ e $\{i, j\} \in \boldsymbol{E}$, diz-se que o enlace $\{i, j\}$ incide nos nós $i$ e $j$. Dois nós ligados por um enlace são ditos nós adjacentes.

\subsubsection{Matrizes de adjacência, laplaciana e normal}

Uma representação natural de um grafo $G(\boldsymbol{V}, \boldsymbol{E})$ é sua matriz de adjacência $\boldsymbol{A}(G)$ :

- 1 na linha $i$, coluna $j$, se o nó $i$ está conectado com o nó $j$;

- 0 em caso contrário.

Para as redes de telecomunicações e muitas outras redes do mundo real, a matriz $\boldsymbol{A}$ é esparsa, ou seja, é constituída de muitos 0 s e poucos 1 s.

De forma mais geral, a matriz de adjacência tanto pode ser usada para grafos binários onde seus elementos não nulos têm o valor 1, quanto para grafos valorados $G(\boldsymbol{V}, \boldsymbol{E}, \boldsymbol{W})$. Nesse caso, denominando $w_{i j}$ os elementos da matriz $\boldsymbol{W}$, a matriz de adjacência $\boldsymbol{A}=\boldsymbol{A}(G)$, é definida como:

$$
A(i, j)= \begin{cases}0 & \text { se } i=j \\ w_{i j} & \text { se } i \text { e } j \text { são nós adjacentes } \\ 0 & \text { nos demais casos }\end{cases}
$$

Para um grafo binário $G(\boldsymbol{V}, \boldsymbol{E})$ o grau de um nó é o número nós adjacentes, isto é, nós vizinhos com os quais tem conexão direta. Assim, o grau de um nó $i$, denotado por $d_{i}$, é o numero de arestas que incidem no nó $i$.

Para um grafo valorado $G(\boldsymbol{V}, \boldsymbol{E}, \boldsymbol{W})$, um conceito equivalente é o peso do nó $i$, denotado por $W_{i}$, que é dado pela soma dos pesos dos enlaces incidentes nesse nó. Em qualquer caso, o grau do nó pode ser determinado somando as linhas (ou colunas) da matriz de adjacência $\boldsymbol{A}$ associada ao grafo.

Seja $\boldsymbol{D}(G)$ uma matriz diagonal $n \times n$, com seus elementos iguais aos graus dos nós $i$ (ou aos pesos do nós $i$ ) do respectivo grafo:

$$
\boldsymbol{D}(i, j)=\left\{\begin{array}{cc}
\sum_{i} w_{i j} & \text { se } i=j \\
0 & \text { se } i \neq j
\end{array}\right.
$$


A chamada matriz laplaciana $\boldsymbol{L}(G)$ para um grafo $G$ é definida como:

$$
\boldsymbol{L}(G)=\boldsymbol{D}(G)-\boldsymbol{A}(G)
$$

A Equação 2.3 pode ser expressa de outra forma como:

$$
\boldsymbol{L}(i, j)= \begin{cases}W_{i} & \text { se } i=j \\ -w_{i j} & \text { se } i \text { e } j \text { são nós adjacentes } \\ 0 & \text { nos demais casos }\end{cases}
$$

No caso de um grafo binário, que é um caso particular de grafo valorado onde todos os pesos $w_{i j}$ assumem apenas os valores 0 ou 1 , os elementos da diagonal da matriz $\boldsymbol{L}$ correspondem aos graus dos nós $d_{i}$.

A chamada matriz normal $N(G)$, que também é conhecida como matriz laplaciana normalizada, laplaciana combinacional ou generalizada ou ainda de $Q$-spectrum, é definida a partir das matrizes $\boldsymbol{A}(G)$ e $\boldsymbol{D}(G)$ através da seguinte expressão:

$$
\boldsymbol{N}(G)=\boldsymbol{I}-\boldsymbol{D}(G)^{-1 / 2} \boldsymbol{A}(G) \boldsymbol{D}(G)^{-1 / 2}
$$

onde $I$ é a matriz identidade de ordem $n \times n$.

A Equação 2.4 pode ser expressa de outra forma, resultando em:

$$
\boldsymbol{N}(i, j)= \begin{cases}1 & \text { se } i=j \\ -w_{i j} W_{i}^{-1 / 2} W_{j}^{-1 / 2} & \text { se } i \text { e } j \text { são nós adjacentes } \\ 0 & \text { nos demais casos }\end{cases}
$$

No caso de um grafo binário, caso particular de um grafo valorado onde todos os pesos $w_{i j}$ assumem apenas os valores 0 ou 1 , os elementos correspondentes a nós adjacentes $i$ e $j$ na matriz $N$ correspondem ao inverso da raiz quadrada do produto dos graus dos nós $d_{i}$ e $d_{j}$.

\subsubsection{Centralidade}

A noção de centralidade provém das ciências sociais. Expressa a importância relativa dos vértices ou arestas dentro de um grafo. No caso de uma rede social, por exemplo, indica o grau de influência dos seus membros [14]. Há diversas classes de centralidade, sendo as mais utilizadas a centralidade de grau, a centralidade de intermediação, a centralidade de proximidade e a centralidade de autovetores, cada uma delas comportando subclasses. 
A centralidade de intermediação pode ser vista como uma medida da influência que um nó tem sobre a propagação do fluxo de informação (ou de qualquer conteúdo) através da rede. É muito usual a medida de centralidade de intermediação de menor caminho (shortest-path betweenness). Para um dado nó $k$, é definida como a probabilidade de que um nó $s$ (origem) tenha de passar pelo nó $k$ para poder chegar ao nó $d$ (destino), utilizando o caminho mais curto possível. A intermediação de menor caminho do nó $k$ para um par de nós $(s, d)$ é dada pela razão entre o número de caminhos entre $s$ e $d$ contendo o nó $k$ e o número total de caminhos entre $s$ e $d$. A intermediação de menor caminho para um enlace $(i, j)$ para um par de nós $(s, d)$ pode ser definida de forma análoga. A intermediação global de um nó (ou enlace) é definida como a soma de todas as intermediações para o nó (ou enlace) para todos os pares de nós $(s, d)$ existentes.

Newman [15] propôs uma medida mais geral, chamada de centralidade de intermediação de percursos aleatórios (random walks), que inclui as contribuições de todos os percursos possíveis na rede e não apenas os caminhos mais curtos. Esta medida considera o fato de que, na realidade, a informação não flui apenas através dos caminhos mais curtos. A medida é baseada em percursos aleatórios, computando de forma probabilística com que frequência um nó é atravessado por percursos aleatórios entre dois outros nós da rede.

Segundo Newman, a intermediação de percursos aleatórios de um nó $k$ para um par origem-destino $(s, d)$ é o valor esperado do número de vezes em que se passa pelo nó $k$ quando se efetua um trajeto aleatório entre a origem $s$ e o destino $d$. A intermediação global do nó $k$ é a soma dessas quantidades, considerando todos os pares $(s, d)$ possíveis.

Em [16], Tizghadam, Li e Leon-Garcia apresentam uma forma de se calcular a intermediação de percursos aleatórios, considerando uma rede definida pelo grafo valorado $G=(\boldsymbol{V}, \boldsymbol{E}, \boldsymbol{W})$. Cada nó tem certa probabilidade de enviar dados aos seus nós adjacentes quando se deseja ir do nó de origem $s$ ao nó de destino final $d$ através de um percurso aleatório. Para um percurso aleatório que parte do nó $s$ a probabilidade de se passar pelo nó $k$ na próxima etapa é representada por $p_{s k d}$, que pode ser expressa por:

$$
p_{s k d}= \begin{cases}0 & \text { se } s=d \\ \frac{w_{s k}}{\sum_{q \in \Phi(s)} w_{s q}} & \text { nos demais casos }\end{cases}
$$


onde $\Phi(s)$ é o conjunto de nós adjacentes a $s$ e $w_{s k}$ é o peso do enlace $(s, k)$, se houver. A primeira condição da Equação 2.5 é devida ao fato de que o nó de destino $d$ é considerado como um nó sumidouro, isto é, qualquer percurso aleatório que chega a esse nó é absorvido, o que leva à condição de $p_{d k d}=0$.

A Equação 2.5 define um sistema markoviano. A intermediação do nó $k$ para um par de nós origem-destino $(s, d)$, representada por $b_{s k d}$, é definida como o valor esperado do número de vezes em que um percurso aleatório entre $s$ e $d$ atravessa o nó $k$. O percurso entre $i$ e $k$ pode ter qualquer comprimento, entre 0 e infinito.

Para fins de cálculos, trata-se o destino $d$ como se fosse um ponto fixo e escreve-se as operações com matrizes baseadas nessa hipótese. Ao final, chega-se de toda forma a um resultado geral, obtendo-se a métrica através da adição dos resultados para todos os destinos possíveis.

Se tabularmos os valores das probabilidades $p_{s k d}$ para o destino $d$ sobre uma matriz $\boldsymbol{P}_{\boldsymbol{d}}$, então, para todo $k \neq d$, a probabilidade de se passar pelo nó $k$ no $q$-ésimo salto, para diferentes valores de $s$ e $k$, pode ser obtida dos elementos correspondentes da matriz. No caso de $k=d$ esse valor seria 0 . Desta forma, a matriz $\boldsymbol{P}_{\boldsymbol{d}}$ pode ser vista como uma matriz de roteamento para o destino $d$ quando um percurso aleatório começa no nó $s$. Utilizandose a relação $\sum_{q} \boldsymbol{P}_{\boldsymbol{d}}=\left(\boldsymbol{I}-\boldsymbol{P}_{\boldsymbol{d}}\right)^{-1}$, pode-se chegar à matriz de intermediação $\boldsymbol{B}_{\boldsymbol{d}}$ para o destino $d$ conforme:

$$
\boldsymbol{B}_{\boldsymbol{d}}=\left[b_{s k \mathrm{~d}}\right]= \begin{cases}\left(\boldsymbol{I}-\boldsymbol{P}_{\boldsymbol{d}}\right)^{-1} & \text { se } k \neq d \\ 0 & \text { nos demais casos }\end{cases}
$$

Examinando a Equação 2.6, pode-se ver que a remoção da linha $d$ e da coluna $d$ tanto na matriz de intermediação $\boldsymbol{B}_{\boldsymbol{d}}$ quanto na matriz de probabilidade $\boldsymbol{P}_{\boldsymbol{d}}$ não afeta os demais elementos. Assim procedendo, a equação 2.6 pode ser reescrita como:

$$
\boldsymbol{B}_{\boldsymbol{d}}(d \mid d)=\left(\boldsymbol{I}-\boldsymbol{P}_{\boldsymbol{d}}(d \mid d)\right)^{-1}
$$

onde $B_{d}(d \mid d)$ e $P_{d}(d \mid d)$ são as matrizes $B_{d}$ e $P_{d}$ truncadas respectivas, resultantes da remoção em ambas da linha $d$ e da coluna $d$.

Da Equação 2.5, considerando que $\sum_{q} w_{s q}$ equivale a $\boldsymbol{D}(d \mid d)$ e que $\left[w_{s k}\right]$ equivale à matriz de adjacência $\boldsymbol{A}(d \mid d)$, pode-se escrever: 


$$
\boldsymbol{P}_{\boldsymbol{d}}(d \mid d)=\left[p_{s k \mathrm{~d}}\right]=\left[w_{s k}\right] \times \boldsymbol{D}^{-1}(d \mid d)=\boldsymbol{A}(d \mid d) \times \boldsymbol{D}^{-1}(d \mid d)
$$

Introduzindo esse resultado na Equação 2.7 chega-se a:

$$
\begin{aligned}
\boldsymbol{I}-\boldsymbol{P}_{\boldsymbol{d}}(\mathrm{d} \mid \mathrm{d}) & =\boldsymbol{I}-\boldsymbol{D}^{-1}(d \mid d) \times \boldsymbol{A}(d \mid d) \\
& =\boldsymbol{D}^{-1}(d \mid d) \times(\boldsymbol{D}(d \mid d)-\boldsymbol{A}(d \mid d))
\end{aligned}
$$

Lembrando que a matriz laplaciana é definida como $\boldsymbol{L}=\boldsymbol{D}-\mathbf{A}$ tem-se:

$$
\boldsymbol{I}-\boldsymbol{P}_{\boldsymbol{d}}(d \mid d)=\boldsymbol{D}^{-1}(d \mid d) \times \boldsymbol{L}(d \mid d)
$$

Substituindo a Equação 2.8 na Equação 2.7 resulta:

$$
\boldsymbol{B}_{d}(d \mid d)=\boldsymbol{L}^{-1}(d \mid d) \times \boldsymbol{D}(d \mid d)
$$

Supõe-se aqui que o grafo $G(\boldsymbol{N}, \boldsymbol{E}, \boldsymbol{W})$ seja conectado. Assim, o inverso da matriz laplaciana reduzida $\boldsymbol{L}(d \mid d)$ existe. Desta forma, a Equação 2.9 tem solução única.

Para se reescrever a Equação 2.8 em termos da matriz laplaciana $\boldsymbol{L}$ do grafo original, sem perda de generalidade, renomea-se os nós de maneira que o nó removido se torne o último nó do grafo $G$ (nó $n$ ). A matriz $\boldsymbol{L}^{-1}(n \mid n)$ pode ser representada em termos de $\boldsymbol{L}$ por meio da matriz pseudo-inversa de Moore-Penrose $\boldsymbol{L}^{+}$, também conhecida como a inversa generalizada da matriz $\boldsymbol{L}$. No presente caso, a pseudo-inversa de Moore-Penrose da matriz $\boldsymbol{L}(n \mid n)$ e sua matriz inversa $\boldsymbol{L}^{-1}(n \mid n)$ são iguais. Isso ocorre porque $\boldsymbol{L}(n \mid n)$ é uma matriz de posto completo (full-rank), pois é uma matriz $(n-1) \times(n-1)$, com posto $(n-$ 1). O posto (rank) de uma matriz é o numero de linhas (ou colunas) linearmente independentes e uma matriz tem posto completo quando seu posto tem o maior valor possível.

Pode-se obter a pseudo-inversa de Moore-Penrose de uma matriz, quando uma coluna é adicionada à matriz original, utilizando a seguinte fórmula recursiva:

$$
\left(M \eta_{p}\right)^{+}=\left(\begin{array}{c}
M^{+}\left(I-\eta_{p} \zeta_{p}\right) \\
\zeta_{p}
\end{array}\right)
$$

onde $\boldsymbol{M}$ é uma matriz com dimensões $p \times q, \boldsymbol{\eta}_{p}$ é um vetor coluna $p \times 1$ e $\zeta_{p}$ é um vetor linha $1 \times p$ tais que:

$$
\zeta_{p}=\left\{\begin{array}{cc}
\left(\boldsymbol{\eta}_{p}-\boldsymbol{M M}^{+} \boldsymbol{\eta}_{p}\right)^{+} & \text {se } \boldsymbol{\eta}_{p} \neq \boldsymbol{M} \boldsymbol{M}^{+} \\
\frac{\left(\boldsymbol{\eta}_{p}{ }^{*}\left(\boldsymbol{M} \boldsymbol{M}^{*}\right)^{+}\right.}{1+\left(\boldsymbol{\eta}_{p}{ }^{*}\left(M M^{*}\right)^{+} \eta_{p}\right.} & \text { se } \boldsymbol{\eta}_{p}=M M^{+}
\end{array}\right.
$$


onde * significa o conjugado transposto ou transposto Hermitiano da matriz.

Para obter $\boldsymbol{L}$ de $\boldsymbol{L}(n \mid n)$, começa-se por adicionar uma coluna a $\boldsymbol{L}(n \mid n)$ para obter:

$$
\boldsymbol{Q}=\left[\boldsymbol{L}(n \mid n) \boldsymbol{z}_{n-1}\right]
$$

O vetor coluna $\boldsymbol{z}_{n-1}$ é escolhido de maneira a fazer com que as somas de todas as linhas da matriz $\boldsymbol{Q}$ sejam iguais a zero. Para satisfazer aos requisitos da matriz laplaciana $\boldsymbol{L}$ deve-se ter:

$$
\left[\boldsymbol{L}(n \mid n) \boldsymbol{z}_{n-1}\right] \overrightarrow{1}_{\mathrm{n}-1}=0
$$

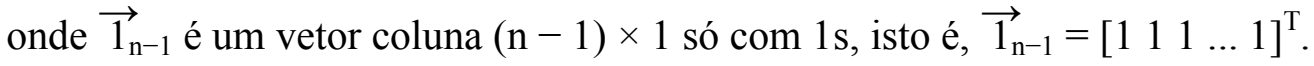

Da Equação 2.13, pode-se ver que:

$$
\begin{aligned}
& \boldsymbol{L}(n \mid n) \overrightarrow{1}_{\mathrm{n}-1}+\mathbf{z}_{\mathrm{n}-1}=0 \\
& \mathbf{z}_{\mathrm{n}-1}=-\boldsymbol{L}(n \mid n) \overrightarrow{1}_{\mathrm{n}-1}
\end{aligned}
$$

Da Equação 2.10 substituindo $\boldsymbol{M}$ por $\boldsymbol{L}(n \mid n)$ e utilizando a Equação 2.14, pode-se ver que:

$$
\begin{aligned}
& \boldsymbol{Q}^{+}=\left(\begin{array}{cc}
\boldsymbol{L}(n \mid n) & \boldsymbol{z}_{n-1}
\end{array}\right)^{+} \\
& =\left(\begin{array}{c}
\boldsymbol{L}^{+}(n \mid n)-\boldsymbol{L}^{+}(n \mid n) \boldsymbol{z}_{n-1} \zeta_{n-1} \\
\zeta_{n-1}
\end{array}\right) \\
& =\left(\begin{array}{c}
\boldsymbol{L}^{+}(n \mid n)+\quad \boldsymbol{L}^{+}(n \mid n) \boldsymbol{L}(n \mid n) \overrightarrow{1}_{\mathrm{n}-1} \zeta_{n-1} \\
\zeta_{n-1}
\end{array}\right) \\
& =\left(\begin{array}{ccc}
\boldsymbol{L}^{+}(n \mid n)^{+} & +\overrightarrow{1}_{\mathrm{n}-1} \zeta_{n-1} \\
& \zeta_{n-1}
\end{array}\right) \\
& =\left(\begin{array}{c}
\boldsymbol{L}^{+}(n \mid n)^{+} \\
0
\end{array}\right)+\overrightarrow{1_{n-1}} \zeta_{n-1}
\end{aligned}
$$

Esta última expressão para $\boldsymbol{Q}^{+}$pode ser expandida na forma:

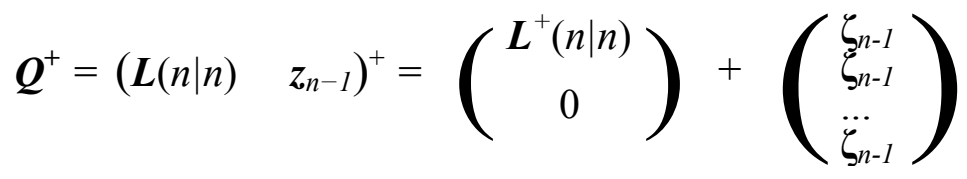



forma:

Os elementos da matriz $\boldsymbol{Q}^{+}$da Equação 2.15 podem ser representados da seguinte

$$
\begin{aligned}
q_{s k}^{+} & =\left(\boldsymbol{L}^{\top}(n \mid n)\right)_{s k}+\left(\boldsymbol{\zeta}_{n-1}\right)_{k} & & \text { se } s \neq n \\
q_{n k}^{+} & =0+\left(\boldsymbol{\zeta}_{n-1}\right)_{k} & & \text { se } s=n
\end{aligned}
$$

Subtraindo a segunda equação da primeira, chega-se a:

$$
\left(\boldsymbol{L}^{+}(n \mid n)\right)_{s k}=q_{s k}^{+}-q_{n k}^{+}
$$

Dando continuidade ao processo para obter $\boldsymbol{L}$ de $\boldsymbol{L}(n \mid n)$, iniciado na Equação 2.12, adiciona-se agora um vetor linha $\boldsymbol{z}_{n}$ ao final da matriz $\boldsymbol{Q}$ para obter-se finalmente a matriz laplaciana $\boldsymbol{L}$, de dimensão $n \times n$ :

$$
\boldsymbol{L}=\left[\begin{array}{l}
\boldsymbol{Q} \\
\boldsymbol{z}_{n}
\end{array}\right]
$$

Utiliza-se mais uma vez a fórmula recursiva da Equação 2.10 aplicada ao transposto dessa matriz, que é igual à própria matriz $\boldsymbol{L}$, considerando que a matriz laplaciana é simétrica. Aplicando um raciocínio similar ao utilizado para se desenvolver as Equações de 2.13 a 2.16, obtém-se:

$$
q^{+}{ }_{s k}=l_{s k}^{+}-l_{s n}^{+}
$$

Utilizando as equações 2.16 e 2.17 pode-se chegar a:

$$
\left(\boldsymbol{L}^{+}(n \mid n)\right)_{\mathrm{sk}}=l_{s k}^{+}-l_{s n}^{+}-l_{n k}^{+}+l_{n n}^{+}
$$

Considerando que $\boldsymbol{L}^{+}(n \mid n)=\boldsymbol{L}^{-1}(n \mid n)$ e trocando o nome do nó de $n$ por $d$ (destino) na Equação 2.18 para deixá-la mais geral, obtém-se assim:

$$
\left(\boldsymbol{L}^{-1}(d \mid d)\right)_{s k}=l_{s k}^{+}-l_{s d}^{+}-l_{d k}^{+}+l_{d d}^{+}
$$

Partindo da Equação 2.9 para a intermediação de percurso aleatório do nó $k$ para o par origem destino $(s, d)$, tem-se:

$$
\begin{aligned}
& B_{d}(d \mid d)=L^{-1}(d \mid d) \times D(d \mid d) \\
& (B \mathrm{~d}(d \mid d))_{s k}=\left(l_{s k}^{+}-l_{s d}^{+}-l_{d k}^{+}+l_{d d}^{+}\right) \times W_{k}
\end{aligned}
$$

Para se obter $b_{k}$, a intermediação de percurso aleatório total do nó $k$, basta somar os resultados para todos os pares origem-destino possíveis: 


$$
\begin{aligned}
& b_{k}=\sum_{s} \sum_{d}\left(B_{d}(d \mid d)\right) \\
& =\sum_{s} \sum_{d} \quad \underline{b_{s k d}} \frac{+b_{d k s}}{2} \\
& \left.=W_{k} \quad \sum_{s} \sum_{d} \quad{\underline{\left(1^{+}\right.}}_{s k}-l^{+} s d-l^{+} d k+l^{+} d d\right)+\left(l^{+} d k-l^{+}{ }_{d s}-l^{+} s k+l^{+} s s\right) \\
& =W_{k} \quad \sum_{s} \sum_{d} \quad \underline{l}^{+} \underline{d d}-l^{+} \underline{s d}-l^{+} \underline{d s}+l^{+} \underline{s} \underline{s}
\end{aligned}
$$

Considerando que tanto a matriz laplaciana quanto sua pseudo-inversa de MoorePenrose são matrizes simétricas, pode-se escrever:

$$
\begin{aligned}
& b_{k}=\frac{W_{k}}{2} \sum_{s} \sum_{d}\left(l_{d d}^{+}+l_{s s}^{+}-2 l_{s d}^{+}\right) \\
& =\frac{W_{k}}{2}\left(\sum_{l_{s d}^{+}} \sum_{d} l_{s s}^{+}+\sum_{s} \sum_{d} l_{d d}^{+}-2 \sum s \sum_{d}\right) \\
& \left.=\frac{W_{k}}{2} \underset{0}{(} \underset{0}{(n s} l_{s s}^{+}+n \sum_{d} l_{d d}^{+}-2 \mathrm{x}\right) \\
& =\frac{W_{k}}{2}\left(2 n \sum_{i} l_{i i}^{+}\right)
\end{aligned}
$$

A soma dos elementos da diagonal principal é o traço da matriz $\boldsymbol{L}^{+}$pseudo-inversa de Moore-Penrose da matriz laplaciana:

$$
b_{k}=W_{k_{-}} n \operatorname{Tr}\left(\boldsymbol{L}^{+}\right)
$$

Um resultado similar pode ser derivado para a intermediação de percurso aleatório para um enlace $(i, j)$ do grafo, com base na soma da intermediação dos dois nós $i$ e $j$ :

$$
\begin{aligned}
b_{i j} & =\frac{1}{2}\left(\frac{w_{i j}}{\sum_{k} w_{i k}} b_{i}+\frac{w_{j i}}{\sum_{k} w_{k j}} b_{j}\right) \\
& =\frac{1}{2}\left(\frac{w_{i j}}{W_{i}} \quad W_{i_{-}} n \operatorname{Tr}\left(\boldsymbol{L}^{+}\right)+\frac{w_{i j}}{W_{j}} \quad W_{j_{-}} n \operatorname{Tr}\left(\boldsymbol{L}^{+}\right)\right. \\
& =\frac{1}{2}\left(w_{i j}+w_{j i}\right) n \operatorname{Tr}\left(\boldsymbol{L}^{+}\right)=w_{i j} \tau \\
& =w_{i j} n \operatorname{Tr}\left(\boldsymbol{L}^{+}\right)
\end{aligned}
$$


Há uma interpretação das Equações 2.20 e 2.21 em termos de sua analogia com circuitos elétricos. Considerando o grafo valorado como um circuito elétrico resistivo, no qual os pesos dos enlaces $w_{i j}$ representam o valor das condutâncias entre os nós nós $i$ e $j$, os fluxos de comunicação nos enlaces fluirão de forma semelhante ao de correntes elétricas nesse circuito resistivo. A intermediação de percurso aleatório total seria o análogo da condutância média (ou o inverso da resistência média) desta rede elétrica.

\subsubsection{Análise espectral}

Um método usual para a análise estatística de dados com múltiplas variáveis é buscar as direções de máxima variabilidade de matrizes, por exemplo, de variançacovariança ou de correlação. Essas direções são chamadas de coordenadas principais ou autovetores, enquanto a grandeza relativa de cada direção é representada por números chamados de autovalores [17]. Esse sistema de coordenadas pode ser encontrado mediante uma serie de rotações, até que o primeiro eixo esteja apontado para a direção de maior variabilidade, o segundo eixo para a direção de maior variabilidade seguinte, ortogonal à primeira, e assim por diante. Como resultado a matriz de dados é reduzida a uma matriz diagonal, com elementos que representam a importância (autovalores ou valores) de cada direção (autovetor ou vetor próprio da matriz).

A coleção de todos os autovalores é chamada de espectro. Um objetivo é reduzir o problema de forma que as dimensões mais importantes, as que têm os maiores autovalores, contenham a maior parte da variabilidade. Implícito nesse método está que uma espécie de sinal esperado ou sinal de fundo foi subtraído da matriz. Por exemplo, no caso de variâncias, isso corresponde à media de cada variável na matriz de dados original.

A análise espectral (decomposição em autovalores e autovetores) faz parte da teoria dos grafos há mais de um século. Sua aplicação ao estudo de redes avançou muito a partir do final dos anos 1960, com o uso de computadores. Os autovalores de uma rede estão associados a propriedades importantes de sua estrutura, tais como seu diâmetro (máximo número de saltos na rede), presença de agrupamentos (clusters), caminhos longos ou à existência de gargalos [18].

Os autovetores, associados aos autovalores, podem ser usados como um sistema de coordenadas para permitir a visualização do grafo. Quando combinados com outros dados do grafo podem ser usados para descrever propriedades importantes de uma rede. 
Definidos formalmente, autovetores e autovalores são uma transformação especial:

$$
\mathrm{T}(\boldsymbol{u})=\lambda \boldsymbol{u}
$$

onde, $\lambda$ é um autovalor (escalar) e $\boldsymbol{u}$ é um autovetor (se $\boldsymbol{u} \geq 0$ ).

Como toda transformação linear pode ser escrita na forma de uma multiplicação de uma matriz por um vetor, pode-se escrever:

$$
\mathrm{T}(\boldsymbol{u})=\boldsymbol{M u}
$$

Das Equações 2.22 e 2.23, tem-se:

$$
\boldsymbol{M u}=\lambda \boldsymbol{u}
$$

que resulta no sistema homogêneo:

$$
(\boldsymbol{M}-\lambda \boldsymbol{I}) \boldsymbol{u}=0
$$

onde se $\boldsymbol{M}$ é uma matriz $n \times \mathrm{x}, \boldsymbol{u}=0$ é sempre uma solução (trivial).

Os vetores $\boldsymbol{u} \geq 0$ para os quais existe um $\lambda$ que resolve a equação 2.24 são chamados de autovetores da matriz $\boldsymbol{M}$ e os valores de $\lambda$, que conjuntamente com $\boldsymbol{u}$ resolvem a equação são chamados de autovalores da matriz $\boldsymbol{M}$ associados aos respectivos autovetores.

Para que a Equação 2.24 tenha solução além da trivial é necessário que o determinante da matriz dos coeficientes seja zero, ou seja,

$$
\operatorname{Det}(\boldsymbol{M}-\lambda \boldsymbol{I})=0
$$

o que resulta em um polinômio de grau $\boldsymbol{n}$ em $\lambda$, conhecido como seu polinômio característico. As raízes do polinômio característico são os autovalores da matriz $\boldsymbol{M}$.

Para se encontrar os autovetores basta substituir o valor do autovalor na equação original e encontrar o autovetor. $\mathrm{O}$ autovalor será, então, associado ao autovetor encontrado.

A Figura 2.2 ilustra em um autovetor $\boldsymbol{u}$ e o autovalor $\lambda$ correspondente. 


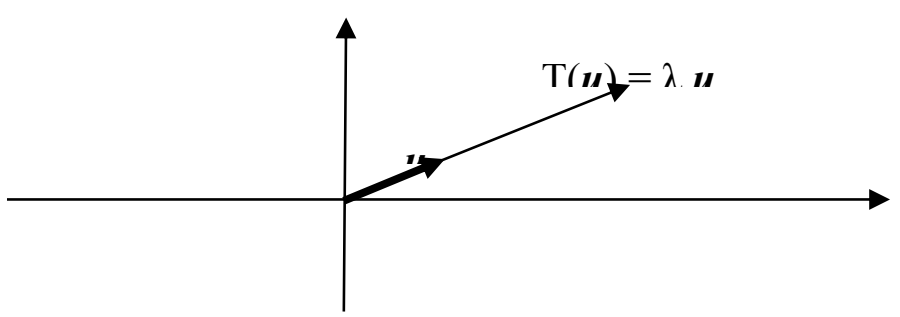

Figura 2.2 - Interpretação gráfica de um autovalor e autovetor

\subsubsection{Invariantes da rede}

Algumas propriedades da matriz de adjacência $\boldsymbol{A}$ não se alteram (são invariantes) sob a série de rotações ortogonais que diagonalizam $\boldsymbol{A}$, isto é, que fazem sua decomposição em autovalores e autovetores. Pode-se relacioná-las com alguns invariantes da rede $\boldsymbol{G}$.

Os autovalores de qualquer matriz simétrica $\boldsymbol{M}$ são as raízes do seu polinômio característico:

$$
X_{n}+c_{1} x^{n-1}+c_{2} x^{n-2}+c_{3} x^{n-3} \ldots+c_{n-1}
$$

Logo, denotando por $\alpha_{\mathrm{i}}$ os autovalores da matriz de adjacência $\boldsymbol{A}$, tem-se:

- $\mathrm{c}_{1}=\alpha_{0}+\alpha_{1}+\ldots+\alpha_{n-1} \quad$ (soma de todos os autovalores de $\mathbf{A}$ );

- $\mathrm{c}_{2}=\alpha_{0} \alpha_{1}+\alpha_{0} \alpha_{2} \ldots+\alpha_{0} \alpha_{n-1} \ldots+\alpha_{n-3} \alpha_{m-1}+\alpha_{n-2} \alpha_{n-1}$ (soma de todos os pares);

- $\mathrm{c}_{3}=\alpha_{0} \alpha_{1} \alpha_{2}+\alpha_{0} \alpha_{1} \alpha_{3}+\ldots+\alpha_{n-3} \alpha_{n-2} \alpha_{n-1}$ (soma de todos os grupos de 3).

O traço de uma matriz é a soma de todos os elementos da sua diagonal e isto é invariante sob rotações ortogonais. Como $\boldsymbol{A}$ tem traço igual a 0 (supondo que não tem laços), tem-se que $\mathrm{c}_{1}=0$.

A soma do produto de pares de autovalores é igual ao número de enlaces, com o sinal invertido, isto é $c_{2}=-|\boldsymbol{E}|$. Uma indicação importante é dada por $\mathrm{c}_{3}$, que corresponde a duas vezes o número de ciclos de 3 . Isso corresponde ao número de triângulos existentes na rede $G$. Coeficientes mais altos estão relacionados com o número de ciclos de 4,5 e assim por diante, ainda que possam incluir também a contribuição de ciclos menores.

Seary e Richards em [19] observam que os autovetores mais importantes de $\boldsymbol{L}$ são os que estão associados aos menores autovalores. Para se chegar aos menores autovalores pode-se subtrair um valor constante da diagonal de $\boldsymbol{L}$ (o que corresponde a subtrair essa constante dos autovalores, sem alterar os autovetores). 
Alguns dos coeficientes do polinômio característico de $\boldsymbol{L}$ são fáceis de se interpretar:

- $\quad \mathrm{c}_{1}=\operatorname{Tr}(\boldsymbol{L})=2|\boldsymbol{E}|$ (isto é, corresponde a duas vezes o número de enlaces)

- $\quad \mathrm{c}_{\mathrm{m}-1}=0$ (considerando que 0 é um autovalor)

- $\left|n \mathrm{c}_{\mathrm{m}-2}\right|=\lambda_{0} \lambda_{1} \ldots \lambda_{\mathrm{n}-1}+\lambda_{0} \lambda_{2} \ldots \lambda_{\mathrm{n}-1}+\ldots+\lambda_{1} \lambda_{2} \ldots \lambda_{\mathrm{n}-1}$

$$
=\lambda_{1} \lambda_{2} \ldots \lambda_{\mathrm{n}-1}
$$

Este último resultado corresponde ao número de árvores geradoras (spanning tree) de $G$, o mesmo que é dado pelo conhecido Teorema Matriz-Árvore (matrix-tree) de Kirchoff, de 1847.

De uma maneira geral, os autovalores de $\boldsymbol{L}$ codificam informações sobre a estrutura de árvores de $G$. O espectro de $\boldsymbol{L}$ contém um 0 para cada componente conectado. Se $\lambda_{1}$ está próximo de 0 , isso indica que o grafo está quase desconectado. Se $\lambda_{1}>>\lambda_{0}$, isto é, se há um grande intervalo (gap) entre os dois primeiros autovalores, isso indica que o diâmetro da rede é pequeno.

Em [20] Seary e Richards analisam o espectro da matriz normal $\boldsymbol{N}$, partindo de sua definição da Equação 2.4, observando igualmente um certo número de invariantes importantes.

Seary e Richards observam ainda que, na tentativa de se encontrar as características globais mais importantes de uma rede, o maior problema para se interpretar o espectro da matriz de adjacência $\boldsymbol{A}$ é a falta de um autovetor "esperado". Esse autovalor esperado existe apenas para grafos $k$-regulares, isto é, grafos onde todos os vértices têm o mesmo grau (o que não corresponde em geral ao caso encontrado em redes reais).

Por outro lado, tanto $\boldsymbol{L}$ quanto $\boldsymbol{N}$ têm sempre um autovetor "esperado", que é o autovetor com apenas 1s, para o qual a interpretação do espectro é clara (ainda que diferente em cada caso). Em outras palavras, tanto $\boldsymbol{L}$ quanto $\boldsymbol{N}$ são funções mais cômodas para representar os grafos que sua matriz de adjacência. 


\subsection{Trabalhos Correlatos}

\subsubsection{Estudos sobre redes}

A origem da teoria dos grafos remonta a 1735, quando Leonhard Euler propôs uma solução para o problema das pontes de Königsberg. O problema consistia em encontrar um caminho que percorresse as sete pontes existentes sobre o rio Pregel passando uma só vez por cada uma delas. Em 1847, Gustav Kirchhoff utilizou a teoria dos grafos para análise de redes elétricas, publicando suas leis sobre circuitos, o que é considerado como a primeira aplicação da teoria dos grafos a um problema de engenharia. Em 1852 Francis Guthrie enunciou o problema que afirma ser possível, utilizando somente quatro cores, colorir qualquer mapa de tal forma que dois países vizinhos nunca tenham a mesma cor. Esse problema, só foi resolvido em 1976. Ao tratar de resolvê-lo, os matemáticos definiram os conceitos teóricos fundamentais para os grafos.

Em 1959, os primeiros trabalhos de Erdös e Renyi modelaram as redes como grafos aleatórios [21], nos quais todos os nós são tratados como estatisticamente equivalentes. Desta forma, os graus dos nós tendem a seguir uma distribuição de Poisson. Este modelo é matematicamente interessante, mas não corresponde bem à maioria das redes do mundo real. Em redes reais, comprimentos de enlaces são geralmente bem mais curtos que os gerados aleatoriamente.

Em 1998 Watts e Strogatz produziram o modelo alfa [22]. Para valores críticos de uma variável $\alpha$ o modelo produz uma rede de tipo "pequeno-mundo", modela adequadamente muitas redes sociais, nas quais há formação de aglomerados (clusters) e o diâmetro da rede tende a ser menor que no modelo de Erdös e Renyi. O modelo alfa era bastante complexo de se analisar, de modo que se introduziu a seguir o modelo beta, que partiu de uma rede constituída por nós com arranjo em anel. Em seguida, substituiu-se as ligações existentes por ligações aleatórias, de acordo com um parâmetro $\beta$. O efeito foi o de produzir uma rede que possui enlaces locais e de longa distância, que modela bem os fenômenos observados em redes sociais e em algumas outras redes.

Em 1999, Barabási e Albert [23] mostraram que, quando os novos nós de uma rede preferem se unir aos nós que já têm muitos enlaces, isso leva a uma distribuição de tipo lei de potência do grau de vértice. Isto é bem distinto da distribuição de graus nas redes aleatórias, que segue uma distribuição de Poisson. O modelo criado por Barabási e Albert ficou conhecido como modelo BA. Muitas redes reais possuem uma distribuição de graus 
que segue o modelo BA. Isso dá origem a redes heterogêneas, com uma distribuição de probabilidade dos graus dos nós variável, sem momentos estatísticos definidos. São assim chamadas de redes "livre-de-escala".

Ainda em 1999, os estudos dos irmãos Faloutsos [24] mostraram que a topologia lógica das redes de telecomunicações que dão suporte a Internet seguem o modelo BA. Segundo esse modelo, a rede se auto organiza ao longo de sua evolução normal e seus nós tendem a se agrupar em torno daqueles que já estão mais conectados. Em [25], Zhao, Park e Lai modelaram as circunstâncias em que uma rede livre-de-escala pode sofrer um colapso, a partir de um certo tipo de ataque que pode produzir falhas em cascata em nós de alta conectividade.

Mais recentemente muitos autores têm trabalhado com modelos de redes complexas em múltiplas camadas, no qual distintas topologias de rede coexistem e interagem. Por exemplo, Halappanavar et al. [26] apresentam um modelo com três camadas, no qual a camada superior é a rede social, a do meio é a rede lógica e a inferior é a infraestrutura física. A análise por camadas permite tratar de forma organizada diferentes aspectos de um mesmo sistema, que são interdependentes.

\subsubsection{Proteção da infraestrutura crítica}

Em julho de 2000, Albert, Jeong e Barabási [27] deixaram evidente a vulnerabilidade das redes de telecomunicações. Eles observaram que a conectividade de redes livres-de-escala, que depende dos nós altamente conectados, tem um preço: a destruição desses nós irá desconectar a rede. Se um atacante remove os nós mais conectados, um após o outro, passado algum ponto limite, o tamanho do maior componente do grafo colapsa, isto é, a rede se quebra em subredes, desconectadas entre si.

Trabalhos de Holme, Kim, Yoon e Han [28], em 2002, estenderam o princípio dos ataques a nós para ataques contra enlaces. Nesse caso, o atacante remove enlaces que conectam os nós de alto grau. De novo, passado algum ponto crítico, a rede se desliga. Eles também sugeriram o uso da centralidade de intermediação como uma alternativa ao grau do vértice na escolha do alvo do ataque. Centralidade de intermediação de um nó é, a grosso modo, a proporção de caminhos em que ele se encontra. Computar a centralidade é um trabalho mais difícil para o atacante do que observar o grau vértice, mas permite-lhe atacar redes onde há pouca ou nenhuma variabilidade no grau dos vértices. 
Depois dos atentados terroristas de 11 de setembro de 2001, muitos estudos sobre vulnerabilidades de redes tem sido feitos [29]. Além das abordagens tradicionais baseadas na conectividade dos nós e na capacidade dos enlaces, metodologias mais complexas, que levam em conta a relação entre elementos físicos e lógicos, têm sido desenvolvidas. Gorman et al. [7] estudaram as vulnerabilidades de redes norte americanas a ataques dirigidos. Propuseram um método que combina suas topologias espaciais física e lógica.

No Brasil, o Centro de Pesquisa e Desenvolvimento - CPqD elaborou várias metodologias para Identificação e Análise da Infraestrutura Crítica (MI2C e MidA2) no âmbito do projeto "Proteção da Infraestrutura Crítica em Telecomunicações PICT" desenvolvido para a Anatel [30]. Estas metodologias se baseiam em questionários sobre as estações respondidos pelas operadoras e na atribuição de pesos (arbitrários) a cada elemento de risco identificado.

A Anatel desenvolveu o projeto "Segurança de Infraestruturas Críticas de Telecomunicações (SIEC)", que deve receber "on-line" informações fornecidas pelas operadoras sobre as estações e rotas utilizadas para a prestação de seus serviços [31]. O SIEC classifica e atribui pesos (arbitrários) aos elementos de risco identificados.

Motta [32] fez um estudo abrangente sobre os backbones de telecomunicações que dão suporte à Internet no Brasil. O conjunto de todas essas redes foi modelado em forma de um grafo. A partir desse grafo, a rede foi analisada, utilizando algumas métricas estruturais, como índices de centralidade e graus de nós, com ênfase particular em seus aspectos geográficos. Em 2004 Alves et al. [33] fizeram uma modelagem lógica da Internet no Brasil, a partir de tabelas extraídas de roteadores de fronteira (BGP). A partir desse modelo fazem uma análise de sua topologia, mostrando que segue o modelo BA (redes livre-de escala) e fazem comparações estatísticas da rede no Brasil com a rede mundial.

Neumayer et al. [34] estudaram o impacto dos desastres geográficas na robustez das redes e sua capacidade de sobrevivência. Eles se concentraram no problema da inibição geográfica da rede, ou seja, como encontrar o corte geográfico mais vulnerável na rede utilizando um segmento de linha ou círculo. As medidas estudadas incluem o grau de nó, a capacidade total esperada e a média de confiabilidade para dois terminais. Trajanovski, Kuipers e Van-Mieghem [35] se aprofundam no problema da inibição geográfica, buscando identificar as regiões críticas de uma topologia de rede. A região crítica é definida como a parte de uma topologia que pode ser fechada por uma figura elementar 
(por exemplo, círculo, retângulo e triângulo equilátero) cuja remoção resultaria na maior interrupção da rede. Eles definiram o par de caminhos disjuntos entre regiões como sendo dois caminhos com pesos mínimos, entre um par origem-destino, tais que não podem falhar ao mesmo tempo por um corte circular.

Agarwal et al. [36] analisaram efeitos de ataques probabilísticos geograficamente correlacionados, nos quais a probabilidade de uma falha dos componentes da rede varia com sua localização geográfica. Eles elaboraram algoritmos para avaliar o efeito das falhas probabilísticas em redes utilizando caminhos de proteção dedicados, com base no número médio de componentes afetados, na confiabilidade média para dois terminais e no fluxo médio depois da falha.

\subsubsection{Estudos sobre métricas}

Em [37] Beguin e Thomas discutem a aplicação a redes de comunicação dos índices $S$ e $I$, originalmente propostos por Ord, em 1967, para caracterizar e classificar redes de transporte. Esses índices são baseados na análise da distribuição de frequências da matriz de caminhos mais curtos associada ao grafo. A comparação entre redes se faz por um gráfico bidimensional com os valores de $S$ e de $I$ nos seus eixos. Conforme sua posição no plano $S-I$, pode-se tirar uma conclusão quanto à distribuição teórica das frequências das distâncias mais curtas de cada rede estudada. Os grafos planares correspondem, em geral, a uma distribuição beta-binomial, enquanto os grafos não-planares levam a uma distribuição hipergeométrica. Os valores de $S$ e $I$ podem ser calculados para uma variedade de distribuições teóricas, baseadas em séries hipergeométricas, e podem ser mapeados no plano $S$ - $I$. O plano $S$ - $I$ resultante forma um arco com uma extremidade indicando que a rede totalmente interconectada (fully meshed) e a outra correspondendo a uma árvore de interconexão mínima (minimally spanning tree). Esses índices, no entanto, não são suficientes para discernir entre duas redes, posto que dois pares de valores de $S$ - $I$ idênticos podem corresponder a redes diferentes.

Em 1973, Fiedler [38] definiu a métrica conhecida como conectividade algébrica. Esta métrica, também chamada de valor de Fiedler de um grafo. A elaboração da métrica parte do teorema Perron-Frobenius aplicado aos autovalores da matriz laplaciana de um grafo finito. O autor observa que o segundo menor entre os autovalores da matriz laplaciana vale zero se e somente se o grafo não está conectado. Além disso, observa que este mesmo autovalor exprime outras propriedades interessantes da conectividade do grafo. 
Jamakovic e Uhlig estudaram a utilização da conectividade algébrica para avaliar a robustez de uma rede a falhas em nós ou enlaces [39]. Efetuaram simulações extensivas em grafos construídos segundo os modelos de Erdös e Renyi (aleatório), de Watts-Strogatz (pequeno mundo) e de Barabási e Albert (livre-de-escala). Chegaram à conclusão de que não há uma relação trivial entre a conectividade algébrica e a robustez da rede.

Tizghadam e Leon-Garcia [40] propuseram uma métrica denominada criticidade da rede para analisar a robustez de uma rede frente a alterações inesperadas na topologia e exigências imprevistas de tráfego. Essa métrica, baseada na intermediação de percursos aleatórios na rede, pode ser calculada para nós e enlaces. A criticidade de rede modela o risco de se tomar um caminho específico, que parte de um nó de origem até um nó de destino. Em [41] os mesmos autores preconizam a utilização dessa métrica em um sistema de gerenciamento de redes que permite oferecer maior robustez a alterações no ambiente operacional, tais como mudanças na topologia ou nos perfis de tráfego.

Em [42] Fay et al. propuseram nova métrica, chamada de espectro ponderado ou distribuição espectral ponderada, que permite a comparação estrutural entre redes. Essa métrica foi aplicada no estudo de sistemas autônomos da Internet como o objetivo de derivar parametrizações ótimas para geradores de topologia em relação a redes reais. Long, Tipper e Gomes, em [43], propuseram utilizar a variação do espectro ponderado como uma métrica para avaliar a capacidade de sobrevivência de redes para falhas geograficamente correlacionadas. Além disso, fazem uma análise comparativa do espectro ponderado com outras métricas, em várias topologias de rede. Chegam à conclusão de que para grafos valorados ou não valorados o espectro ponderado proposto é mais versátil que as outras métricas analisadas.

Em [44], Liu, Pawlikowski e Sirisena estudaram o uso da conectividade algébrica para determinar a distribuição ótima da capacidade de reserva disponível em caso de falha de elementos de rede. Fizeram um estudo muito abrangente, utilizando como métricas a distância média (valor médio entre os caminhos mais curtos) e a conectividade algébrica, bem como uma composição entre ambas. A métrica composta foi utilizada para comparar diversas redes, de distintos tamanhos e topologias.

Um trabalho de Bigdeli, Tizghadam e Leon-Garcia [45] trata da comparação entre múltiplas métricas. Nesse estudo foram analisados os comportamento de diferentes métricas estruturais e espectrais aplicadas em algumas topologias de grafos bem 
conhecidas. Examinaram em particular o efeito do tamanho da rede. Foram comparadas a criticidade de rede, a conectividade algébrica, o grau médio e a centralidade média de nó. Chegam à conclusão de que não há uma métrica única e universal que possa satisfazer tanto os objetivos de conectividade quanto de robustez, mantendo uma complexidade razoável. Os autores observam que cada métrica capta apenas alguns atributos do grafo.

Como visto acima, há várias modelagens possíveis para as redes. Uma profusão de métricas de conectividade, em domínios estruturais e espectrais, têm sido propostas. Cada métrica captura diferentes características dos grafos e possui seus domínios de aplicação específicos. Para capturar diferentes características dos grafos, recorre-se a uma seleção de métricas detalhadas no capítulo seguinte. A idéia é explorar métricas de distintas classes entre as mais citadas em trabalhos recentes envolvendo redes de telecomunicações. 


\section{MÉTRICAS DE CONECTIVIDADE}

Neste capítulo, analisa-se as principais métricas que poderiam ser usadas como indicadores para avaliar a vulnerabilidade das redes, sob distintos aspectos. Assim, seleciona-se uma boa diversidade de métricas que quantificam o impacto na capacidade de sobrevivência em termos de conectividade, em caso de perda de nós ou enlaces de uma rede.

Apesar de todos os trabalhos feitos, ainda não foi encontrada uma métrica abrangente e universal, aplicável às redes reais de telecomunicações, que seja adequada a quaisquer topologias e circunstâncias de uso dessa rede. Considerando que cada métrica capta apenas alguns aspectos do grafo, o tratamento dos múltiplos atributos, tais como os envolvidos na avaliação da vulnerabilidade das redes, continua sendo um problema em aberto. Desta forma parece judicioso testar um grande número de métricas nos modelos de rede de interesse, antes de se escolher uma delas, adotar várias ou de se fazer uma combinação adequada entre elas.

\subsection{Dados de base}

Para a obtenção das medidas é necessário contar com um conjunto de dados básicos da rede de interesse, conforme se explica a seguir. Para melhor ilustrar os procedimentos relativos às métricas, o grafo mostrado na Figura 3.1 será utilizado como exemplo.

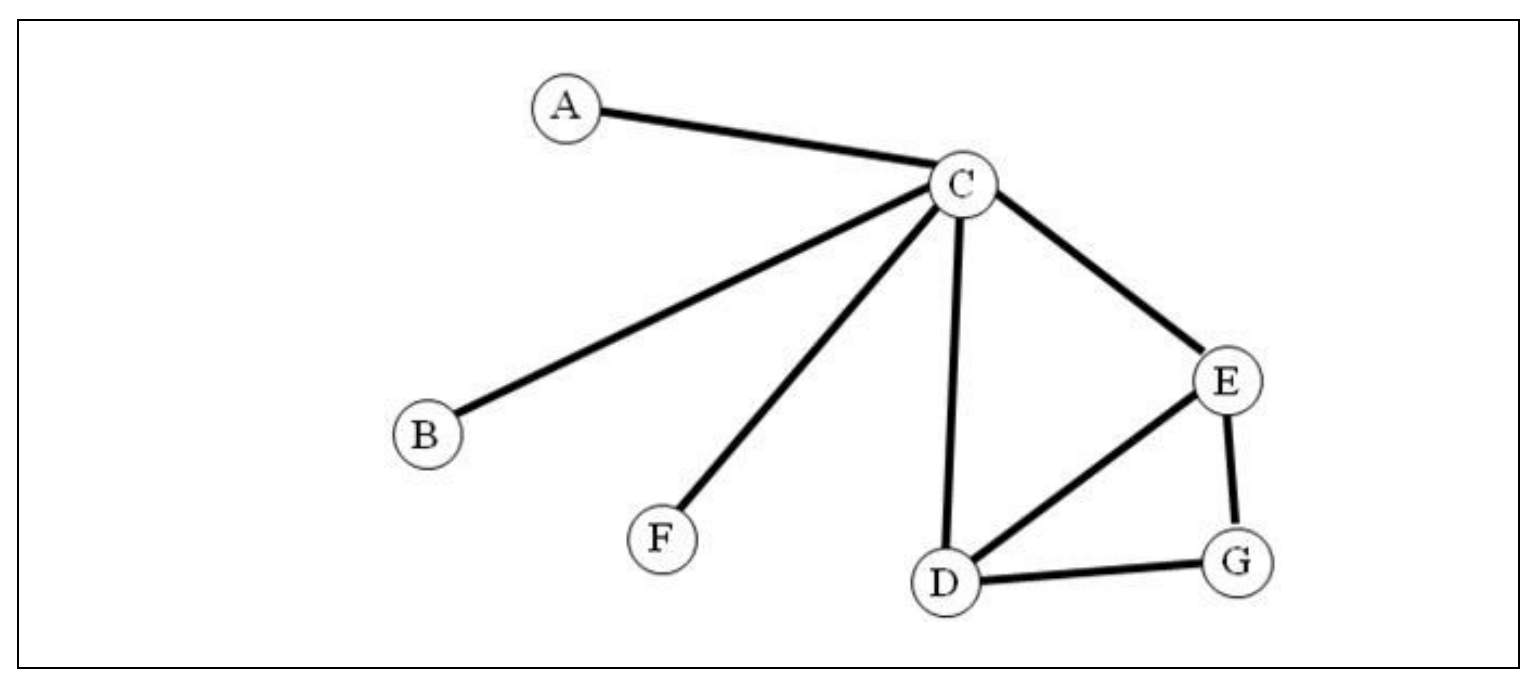

Figura 3.1 - Exemplo usado para ilustrar o cálculo das métricas

Essa rede possui 7 nós e 8 enlaces, que podem ser completamente representados pelas Tabelas 3.1 e 3.2 . 
Tabela 3.1 - Nós do grafo da Figura 3.1, utilizado como exemplo

\begin{tabular}{|c|c|c|}
\hline Nó & Descrição & Sigla \\
\hline 1 & Nó_A & A \\
2 & Nó_B & B \\
3 & Nó_C & C \\
4 & Nó_D & D \\
5 & Nó_E & E \\
6 & Nó_F & F \\
7 & Nó_G & G \\
\hline
\end{tabular}

Tabela 3.2 - Enlaces no grafo da Figura 3.1, utilizado como exemplo

\begin{tabular}{|cl|c|}
\hline Enlace & Sigla \\
\hline 1 & 3 & A-C \\
2 & 3 & B-C \\
3 & 4 & C-D \\
3 & 5 & C-E \\
3 & 6 & C-F \\
4 & 5 & D-E \\
4 & 7 & D-G \\
5 & 7 & E-G \\
6 & 7 & E-G \\
\hline
\end{tabular}

A partir dessas duas tabelas, constrói-se facilmente a matriz de adjacência $\boldsymbol{A}$ da rede $G$ conforme definida na Seção 2.1. Utilizando-se a Equação 2.1, chega-se ao resultado mostrado na Tabela 3.3:

Tabela 3.3 - Representação da matriz de adjacência

\begin{tabular}{|c|ccccccc|}
\hline Nó & A & B & C & D & E & F & G \\
\hline A & 0 & 0 & 1 & 0 & 0 & 0 & 0 \\
B & 0 & 0 & 1 & 0 & 0 & 0 & 0 \\
C & 1 & 1 & 0 & 1 & 1 & 1 & 0 \\
D & 0 & 0 & 1 & 0 & 1 & 0 & 1 \\
$\mathbf{E}$ & 0 & 0 & 1 & 1 & 0 & 0 & 1 \\
F & 0 & 0 & 1 & 0 & 0 & 0 & 0 \\
$\mathbf{G}$ & 0 & 0 & 0 & 1 & 1 & 0 & 0 \\
\hline
\end{tabular}

A partir da matriz de adjacência constrói-se a matriz laplaciana $\boldsymbol{L}$ por meio da Equação 2.3. Está ilustrada na Tabela 3.4, para o exemplo da Figura 3.1.

Tabela 3.4 - Representação da matriz laplaciana

\begin{tabular}{|c|rrrrrrr|}
\hline Nó & A & B & C & D & E & F & G \\
\hline A & 1 & 0 & -1 & 0 & 0 & 0 & 0 \\
B & 0 & 1 & -1 & 0 & 0 & 0 & 0 \\
C & -1 & -1 & 5 & -1 & -1 & -1 & 0 \\
\hline
\end{tabular}




\begin{tabular}{|c|ccrrrrr|}
\hline Nó & A & B & C & D & E & F & G \\
\hline D & 0 & 0 & -1 & 3 & -1 & 0 & -1 \\
E & 0 & 0 & -1 & -1 & 3 & 0 & -1 \\
F & 0 & 0 & -1 & 0 & 0 & 1 & 0 \\
G & 0 & 0 & 0 & -1 & -1 & 0 & 2 \\
\hline
\end{tabular}

A partir desta matriz obtém-se o espectro laplaciano, constituído de todos os autovalores $\lambda_{i}$ da matriz laplaciana $\boldsymbol{L}$. Os 7 autovalores (calculados utilizando MATLAB) associados à matriz da Tabela 3.4 são apresentados na Tabela 3.5.

Tabela 3.5 - Representação do espectro laplaciano

\begin{tabular}{|c|ccccccc|}
\hline Autovalor & $\lambda_{0}$ & $\lambda_{1}$ & $\lambda_{2}$ & $\lambda_{3}$ & $\lambda_{4}$ & $\lambda_{5}$ & $\lambda_{6}$ \\
\hline valor calculado & 0 & 0,73 & 1 & 1 & 3,14 & 4 & 6,13 \\
\hline
\end{tabular}

Da mesma forma, a partir da matriz de adjacência $\boldsymbol{A}$ da Tabela 3.3 é construída a matriz normal $\boldsymbol{N}$ por meio da Equação 2.4. Para o caso do exemplo da Figura 3.1 esta matriz está mostrada na Tabela 3.6.

Tabela 3.6 - Representação da matriz normal

\begin{tabular}{|c|ccccccc|}
\hline Nó & $\mathbf{A}$ & $\mathbf{B}$ & $\mathbf{C}$ & $\mathbf{D}$ & $\mathbf{E}$ & $\mathbf{F}$ & $\mathbf{G}$ \\
\hline $\mathbf{A}$ & 1 & 0 & $-0,45$ & 0 & 0 & 0 & 0 \\
$\mathbf{B}$ & 0 & 1 & $-0,45$ & 0 & 0 & 0 & 0 \\
$\mathbf{C}$ & $-0,45$ & $-0,45$ & 1 & $-0,26$ & $-0,26$ & $-0,45$ & 0 \\
$\mathbf{D}$ & 0 & 0 & $-0,26$ & 1 & $-0,33$ & 0 & $-0,41$ \\
$\mathbf{E}$ & 0 & 0 & $-0,26$ & $-0,33$ & 1 & 0 & $-0,41$ \\
$\mathbf{F}$ & 0 & 0 & $-0,45$ & 0 & 0 & 1 & 0 \\
$\mathbf{G}$ & 0 & 0 & 0 & $-0,41$ & $-0,41$ & 0 & 1 \\
\hline
\end{tabular}

A partir desta matriz obtém-se o espectro normal, constituído de todos os autovalores $v_{i}$ da matriz normal $N$. De maneira similar, os 7 autovalores associados à matriz da Tabela 3.6 (calculados utilizando MATLAB) são apresentados na Tabela 3.7.

Tabela 3.7 - Representação do espectro normal

\begin{tabular}{|c|ccccccc|}
\hline Autovalor & $v_{0}$ & $v_{1}$ & $v_{2}$ & $v_{3}$ & $v_{4}$ & $v_{5}$ & $v_{6}$ \\
\hline valor calculado & 0 & 0,41 & 1 & 1 & 1,33 & 1,39 & 1,86 \\
\hline
\end{tabular}




\subsection{Métricas estruturais}

\subsubsection{Grau médio dos nós}

O número de nós e o número de enlaces são os parâmetros mais importantes de qualquer rede $G(\boldsymbol{V}, \boldsymbol{E})$. A métrica grau médio dos nós $A D$ (average degree) depende apenas da quantidade de nós $|\boldsymbol{V}|$ e da quantidade de enlaces $|\boldsymbol{E}|$. Partindo-se da matriz $\boldsymbol{D}(G)$ definida pela Equação 2.2, pode-se mostrar que a média entre os graus dos nós que estão em sua diagonal principal, para uma rede com $|\boldsymbol{V}|$ nós e $|\boldsymbol{E}|$ enlaces, é dado por:

$$
A D=\frac{1}{\boldsymbol{n}} \sum_{i} d_{i}=\frac{2|\boldsymbol{E}|}{|\boldsymbol{V}|}
$$

Uma variante dessa métrica é a densidade de enlaces, indicada como $\rho$, é definida como a razão entre o número de enlaces $|\boldsymbol{E}|$ e o número máximo de par de nós que poderiam ser conectados diretamente em uma dada rede:

$$
\rho=\frac{2|\boldsymbol{E}|}{n(n-1)}
$$

Um maior valor de $A D$ indica que, em média, há um maior número de enlaces incidentes em cada nó. Assim, a conectividade da rede é mais forte. Logo, menor será sua vulnerabilidade em caso de falhas ou ataques.

Para o caso do exemplo da Figura 3.1, pode-se ver na Tabela 3.1 que $|\boldsymbol{V}|=7$ (são 7 nós) e na Tabela 3.2 que $|\boldsymbol{E}|=8$ (são 8 enlaces). Assim, pode-se obter:

$$
A D=2|\mathrm{E}| /|\mathrm{V}|=2 \times 8 / 7=2,286
$$

Essa métrica é amplamente utilizada. Entretanto, é uma métrica bastante grosseira, posto que não reflete diferenças evidentes. No caso do grafo da Figura 3.1, por exemplo, se incluímos um novo enlace entre os nós A e B, ao mesmo tempo em que se retira o enlace existente entre os nós $\mathrm{C}$ e $\mathrm{D}$, a topologia da rede muda bastante, mas o grau médio dos nós não se altera.

\subsubsection{Diâmetro da rede}

Sendo $\boldsymbol{H}(G)$ a matriz com o número mínimo de saltos (hops) entre todos os pares de nós $(i, j)$ da rede $G$, a métrica diâmetro da rede $D I A$ é definida como o valor máximo de $h_{i j}$. O número de saltos $h_{i j}$ é sempre contado sobre o caminho mais curto entre cada par de nós. O caminho mais curto entre um par de nós $(i, j)$ e o $h_{i j}$ respectivo podem ser calculados por 
algoritmos de mínima distância, como o algoritmo de Djiskstra [46], por exemplo. Daí, pode-se construir a matriz $\boldsymbol{H}(G)$, também conhecida como a matriz de distâncias mínimas de $G$, a partir da matriz de adjacência $A$. Assim, tem-se:

$$
D I A=h_{\max }=\max \left\{h_{i j}: h_{i j} \in \boldsymbol{H}(G)\right\}
$$

No caso do exemplo da Figura 3.1, a partir da matriz de adjacência $\boldsymbol{A}$ da Tabela 3.3 pode-se obter a matriz das distâncias mínimas. O resultado (calculado com o algoritmo de Dijkstra, utilizando MATLAB) é mostrado na Tabela 3.8.

Tabela 3.8 - Representação da matriz de distâncias mínimas

\begin{tabular}{|c|ccccccc|}
\hline Nó & A & B & C & D & E & F & G \\
\hline A & 0 & 2 & 1 & 2 & 2 & 2 & 3 \\
B & 2 & 0 & 1 & 2 & 2 & 2 & 3 \\
C & 1 & 1 & 0 & 1 & 1 & 1 & 2 \\
D & 2 & 2 & 1 & 0 & 1 & 2 & 1 \\
$\mathbf{E}$ & 2 & 2 & 1 & 1 & 0 & 2 & 1 \\
F & 2 & 2 & 1 & 2 & 2 & 0 & 3 \\
G & 3 & 3 & 2 & 1 & 1 & 3 & 0 \\
\hline
\end{tabular}

O diâmetro da rede é o valor máximo entre os elementos da matriz de distâncias mínimas. Na Tabela 3.8, vê-se que o valor máximo é 3. Assim, o diâmetro da rede no caso do exemplo da Figura 3.1 é:

$$
D I A=3
$$

Para redes com o mesmo número de nós e enlaces, quanto menor o valor de $D I A$, mais forte será a conectividade da rede e menor será sua vulnerabilidade esperada.

\subsubsection{A distância média}

A distância média $A S P$ (average shortest path) é definida como a média entre o número mínimo de saltos $h_{i j}$ entre todos os pares de nós da rede $G$, que são os elementos da matriz de distâncias mínimas $H(G)$. Para o cálculo dessa média considera-se apenas os elementos situados acima da diagonal principal, já que aqui se trabalha apenas com grafos não orientados. Isso resulta na fórmula:

$$
A S P=\sum_{k=1}^{D I A} \quad k \cdot \operatorname{Pr}\left[h_{i j}=k\right] \frac{1}{2 n(n-1)} \quad \sum_{i, j} h_{i j}
$$

A Equação 3.3 diz que a distância média pode ser calculada pela soma de todos os elementos da matriz de distâncias mínimas, dividida por $2 n(n-1)$, que corresponde ao total 
de pares de nós distintos possíveis nessa matriz (número de elementos fora da diagonal principal). No caso do exemplo da Tabela 3.8, soma de todos os elementos da matriz resulta em 74. Duas vezes o número total de pares de nós distintos corresponde a $2 \times 7 \times 6=84$. A distância média é o quociente entre esses dois valores:

$$
A S P=73 / 84=0,88
$$

A distância média é uma métrica aparentada ao diâmetro da rede, mas um tanto mais sensível. De maneira similar ao diâmetro, para redes com o mesmo número de nós e enlaces, quanto menor for o valor de $A S P$, mais forte será a conectividade. Logo, menor será a vulnerabilidade esperada para essa rede.

\subsection{4 Índices $S$-I}

Como visto na Seção 2, os índices S e I foram originalmente propostos para caracterizar e classificar redes de transporte. Esses índices são baseados na distribuição de frequências $\boldsymbol{f}$ construída a partir da matriz de distâncias mínimas $\boldsymbol{H}(G)$ associada ao grafo. Utilizando-se um gráfico bidimensional no qual são representados os valores de $S$ e de $I$ nos seus eixos pode-se obter uma indicação quantitativa de quão desconectada esta rede se torna na medida em que seus nós ou enlaces são removidos.

$S$ e $I$ dependem dos três primeiros momentos da distribuição dos caminhos mais curtos em uma dada rede $G$ : a média, o desvio padrão e a obliquidade (skewness).

$\mu=$ média da distribuição de frequências $\boldsymbol{f}$ de distâncias mínimas

$\sigma=$ desvio padrão da distribuição de frequências $f$

$\gamma=$ obliquidade (skewness) da distribuição de frequências $\boldsymbol{f}$

Os índices são assim definidos:

$$
\begin{aligned}
& S=\gamma / \mu \\
& I=\sigma / \mu
\end{aligned}
$$

Vê-se que o índice $\mathrm{S}$ está relacionado com o grau de simetria da distribuição de frequências de distâncias mínimas $\boldsymbol{f}$, enquanto o índice I corresponde ao coeficiente de variação de Pearson, que é uma medida de dispersão relativa obtida pela razão entre o desvio-padrão e a média. 
A partir dessa matriz de distâncias mínimas $\boldsymbol{H}(G)$ de dimensão $n \times n$, um vetor linha $\boldsymbol{f}$ que representa a distribuição de frequência do número de saltos nos caminhos mais curtos, que varia de 0 até o diâmetro da rede, pode ser construído. No caso do exemplo da Tabele 3.8, o resultado está mostrado na Tabela 3.9:

Tabela 3.9 - Distribuição da frequência do número de saltos nos caminhos mais curtos

\begin{tabular}{|c|cccc|}
\hline Número de saltos & $\mathbf{0}$ & $\mathbf{1}$ & $\mathbf{2}$ & $\mathbf{3}$ \\
\hline Frequência $\boldsymbol{f}$ & 14 & 20 & 16 & 6 \\
\hline
\end{tabular}

A partir da distribuição de frequências do número mínimo de saltos da Tabela 3.9, pode-se calcular (utilizando MS-Excel ou MATLAB):

$$
\begin{aligned}
& \mu=14 \text { (média) } \\
& \sigma=5,89 \text { (desvio padrão) } \\
& \gamma=-0,94 \text { (obliquidade) }
\end{aligned}
$$

E também calcula-se os índices:

$$
\begin{array}{ll}
S=\gamma / \mu & =-0,16 \\
I=\sigma / \mu & =0,42
\end{array}
$$

A comparação entre redes se faz por um gráfico bidimensional com os valores de $\mathrm{S}$ e de I nos seus eixos. O plano S-I resultante forma um arco, com uma extremidade indicando rede totalmente interligada (fully meshed) e a outra uma árvore de interconexão minima (minimally spanning tree). Conforme sua posição no plano S-I, pode-se tirar uma conclusão quanto à distribuição teórica das frequências das distâncias mais curtas de cada rede. O exemplo em pauta está próximo da interconexão mínima.

\subsubsection{Criticidade da rede}

A criticidade de rede é definida como a razão entre a intermediação de percurso aleatório (vista na Seção 2.2.2) e a soma dos pesos envolvidos (no caso de grafos não valorados todos os pesos são iguais a 1). Pode ser calculada a partir da centralidade de um nó ou, alternativamente, a partir da centralidade de um enlace, chegando-se ao mesmo resultado. Assim, criticidade de rede $\tau$ pode ser calculada a partir de um nó $k$ qualquer, através da expressão:

$$
\tau=\underline{b}_{\underline{k}}
$$


onde $b_{k}$ é a a intermediação de percurso aleatório do nó e $W_{k}$ é peso do nó, definido como a soma dos pesos de todos os enlaces nele incidentes.

Utilizando a Equação 2.20, que expressa a intermediação de percurso aleatório para nós $b_{k}$ em termos de $W_{k}$ e da matriz laplaciana $\boldsymbol{L}$, a criticidade de rede $\tau$ corresponde a:

$$
\tau=\frac{b_{k}}{W_{k}}=2 n \operatorname{Tr}\left(\boldsymbol{L}^{+}\right)
$$

onde $\boldsymbol{L}^{+}$é o inverso de Moore-Penrose da matriz laplaciana $\boldsymbol{L}$ e $n$ é o número de nós do grafo $G$. Observa-se na expressão acima que a criticidade de rede $\tau$ é uma quantidade global da rede, independente da posição de quaisquer nós ou enlaces específicos utilizados para o seu cálculo.

A métrica aqui usada será a criticidade de rede normalizada, definida como:

$$
N C=\tau / \mathrm{n}(\mathrm{n}-1)=2 \operatorname{Tr}\left(\boldsymbol{L}^{+}\right) /(n-1)
$$

Há uma interpretação interessante de $N C$, em termos de circuitos elétricos. A criticidade de rede corresponderia à média não ponderada das resistências equivalentes em uma rede onde os enlaces fossem resistores. Logo, otimizar a criticidade de rede equivale a minimizar a resistência média, ou seja, maximizar a condutância média da rede.

Calculando a pseudo-inversa de Moore-Penrose $\boldsymbol{L}^{+}$para o exemplo da Figura 3.1, utilizando sua matriz laplaciana $\boldsymbol{L}$ da Tabela 3.4, chega-se à Tabela 3.10 abaixo:

Tabela 3.10 - Pseudo-inversa de Moore-Penrose da matriz laplaciana

\begin{tabular}{|l|rrrrrrr|}
\hline Nó & \multicolumn{1}{|c}{ A } & \multicolumn{1}{c}{ B } & \multicolumn{1}{c}{ C } & \multicolumn{1}{c}{ D } & \multicolumn{1}{c}{ E } & \multicolumn{1}{c|}{ F } & \multicolumn{1}{c|}{ G } \\
\hline A & 0,88 & $-0,12$ & 0,02 & $-0,19$ & $-0,19$ & $-0,12$ & $-0,27$ \\
$\mathbf{B}$ & $-0,12$ & 0,88 & 0,02 & $-0,19$ & $-0,19$ & $-0,12$ & $-0,27$ \\
$\mathbf{C}$ & 0,02 & 0,02 & 0,16 & $-0,05$ & $-0,05$ & 0,02 & $-0,12$ \\
$\mathbf{D}$ & $-0,19$ & $-0,19$ & $-0,05$ & 0,36 & 0,11 & $-0,19$ & 0,16 \\
$\mathbf{E}$ & $-0,19$ & $-0,19$ & $-0,05$ & 0,11 & 0,36 & $-0,19$ & 0,16 \\
$\mathbf{F}$ & $-0,12$ & $-0,12$ & 0,02 & $-0,19$ & $-0,19$ & 0,88 & $-0,27$ \\
$\mathbf{G}$ & $-0,27$ & $-0,27$ & $-0,12$ & 0,16 & 0,16 & $-0,27$ & 0,59 \\
\hline
\end{tabular}

$\mathrm{Na}$ Tabela 3.10, nota-se que o traço (soma dos elementos da diagonal principal) da matriz $\boldsymbol{L}+$ corresponde a 4,11. Para se obter a criticidade de rede, basta multiplicar o traço por 2 e dividir por ( $n$-1), que neste caso é igual a 6 , obtendo:

$$
N C=2 \times 4,11 / 6=1,37
$$


Quanto menor for $N C$, menos sensível a mudanças de topologia e a variações de tráfego será a rede.

\subsection{Métricas espectrais}

\subsubsection{Conectividade Algébrica}

A primeira métrica espectral aqui usada é a conectividade algébrica $A C$. Quanto maior $A C$, mais difícil será quebrar a rede em subredes desconectadas. Logo, sua vulnerabilidade a falhas e ataques será menor. Como visto na Seção 2, a conectividade algébrica $A C$ deriva do espectro Laplaciano, i.e., dos autovalores $\lambda_{0}, \lambda_{1}, \ldots \lambda_{N}$ da matriz $\boldsymbol{L}(G)$. O menor autovalor $\lambda_{0}$ é sempre zero. Os demais serão sempre positivos, salvo se a rede for desconectada. $A C$ é o segundo menor autovalor $\lambda_{1}(G)$, também conhecido como valor de Fiedler:

$$
A C=\lambda_{1}(G)
$$

Para o grafo da Figura 3.1 utilizado como exemplo, a conectividade algébrica pode ser extraída diretamente da Tabela 3.3 dos autovalores da matriz laplaciana:

$$
\mathrm{AC}=0,73
$$

Conforme visto no Capítulo 2, a conectividade algébrica de um grafo pode ser utilizada na análise de vulnerabilidade de redes. O objetivo da análise de vulnerabilidade é reduzir o risco em relação aos incidentes de segurança, antecipando possíveis falhas que possam vir a comprometer a rede. Face a este conceito, pode-se medir a importância de um nó em relação ao impacto que causa na rede, caso venha a ser dela retirado.

Em [39] mostra-se que a conectividade algébrica $\lambda_{I}(G)$ da rede $G$ pode ser utilizada para caracterizar a robustez dessa rede com respeito à sua conectividade topológica. Foi demonstrado que a conectividade algébrica é igual a 0 , apenas se a rede $G$ está desconectada. Se mais valores próprios da matriz Laplaciana $\boldsymbol{L}(G)$ são iguais a zero, então o número de tais autovalores indica o número de subredes desconexas de $G$. Foi também mostrado que quanto maior for a conectividade algébrica de uma rede, mais difícil será quebrar a rede em componentes desconexos. Assim, a conectividade algébrica é uma métrica comumente aceita para estudar propriedades, tais como a conectividade e resultados de cortes em redes. 
Assim, através da utilização da conectividade algébrica de redes, pode-se quantificar a importância de um nó ou enlace específico em uma determinada topologia, pela comparação da conectividade algébrica antes e após a falha do nó ou do enlace específico. Os nós ou enlaces mais importantes, sob a perspectiva de sobrevivência da rede, são aqueles que causam a redução maior na conectividade algébrica da rede ao falharem. Assim, tais nós ou enlaces precisam de mais proteção, para garantir que a conectividade da rede continue a ser tão grande quanto possível.

\subsubsection{Espectro ponderado}

Como visto na Seção 2, a métrica denominada de espectro ponderado WS foi originalmente proposta para analisar a topologia da Internet. No entanto, em [43] Long et al. sugeriram usar a máxima variação de $W S$ para quantificar a capacidade de sobrevivência de uma rede. WS é baseada no espectro normal, isto é, nos autovalores $v_{0}, v_{1}$, $\ldots v_{n-1}$ da matriz laplaciana normalizada $N(G)$. Essa métrica é dada pela expressão:

$$
W S=\sum_{i}\left(1-v_{\mathrm{i}}\right)^{\varepsilon}
$$

A propriedade da rede medida depende do expoente $\varepsilon$ escolhido. Com $\varepsilon=3$ tem-se ciclos de três, que medem o número de triângulos no grafo. Assim, $\varepsilon=3$ está relacionado com o coeficiente de aglomeração (clustering). Com ciclos de quatro, isto é, $\varepsilon=4$, se mede o número de caminhos disjuntos na rede. Quanto mais caminhos disjuntos entre nós houver na rede, menor será sua vulnerabilidade à remoção de nós ou enlaces. Assim, no presente trabalho, utiliza-se este valor para $\varepsilon$, o que resulta em:

$$
W S 4=\sum_{i}\left(1-v_{\mathrm{i}}\right)^{4}
$$

Para o grafo da Figura 3.1 utilizado como exemplo, utilizando os autovalores da Tabela 3.7 chega-se aos elementos mostrados na Tabela 3.11:

Tabela 3.11 - Cálculo de WS4

\begin{tabular}{|c|ccccccc|}
\hline termo & $\left(1-v_{0}\right)^{4}$ & $\left(1-v_{1}\right)^{4}$ & $\left(1-v_{2}\right)^{4}$ & $\left(1-v_{3}\right)^{4}$ & $\left(1-v_{4}\right)^{4}$ & $\left(1-v_{5}\right)^{4}$ & $\left(1-v_{6}\right)^{4}$ \\
\hline valor & 1 & 0,12 & 0 & 0 & 0,012 & 0,02 & 0,55 \\
\hline
\end{tabular}

A soma de todos os elementos resulta em:

$$
W S 4=1,71
$$

O último autovalor $\left(v_{6}\right)$ é o mais crítico para o cáculo do espectro ponderado. 


\subsection{Métricas compostas}

Liu, Pawlikowski e Sirisena propuseram em [44] uma métrica composta para comparar redes de diferentes topologias, tanto em termos do seu tamanho absoluto quanto de sua forma. A métrica por eles proposta seria capaz de conviver com diferentes topologias de rede, quaisquer que sejam suas dimensões e formas. Para isso combinaram uma métrica estrutural, a distância média $A S P$, com uma métrica espectral, a conectividade algébrica $A C$. A métrica composta por eles concebida para quantificar topologias arbitrárias e dada pelo quociente entre a distância média $A S P$ e a conectividade algébrica $A C$. Para testar esta métrica composta foram utilizadas 20 topologias distintas.

No presente estudo busca-se utilizar uma métrica composta mais abrangente para combinar os efeitos de múltiplos atributos. Além das métricas $A C$ (dificuldade de se quebrar a rede em subredes desconectadas) e $A S P$ (distância média entre nós), a ideia é também incorporar outros aspectos das redes, tais como os efeitos da capacidade de acomodar variações no perfil de tráfego (métrica $N C$ ) e a influência do número de caminhos disjuntos (métrica WS4). Para combinar múltiplos atributos, recorre-se à Teoria de Utilidade Multicritério (Multiple Attribute Utility Theory - MAUT). Esta teoria oferece métodos para a construção de uma função de utilidade e um critério de ponderação de pesos para as várias métricas aqui consideradas.

De uma forma mais geral, a Teoria da Utilidade, estudada em Pesquisa Operacional, permite a representação das preferências relativas entre os elementos de um conjunto, usando-se números reais para representá-los. A Teoria de Utilidade Multicritério (MAUT), deriva da Teoria da Utilidade, permitindo tratar de problemas com múltiplos objetivos [47]. Esta teoria supõe que todos os atributos são comparáveis e que existe transitividade nas relações de preferência ou de indiferença entre atributos. Oferece um método adequado a tratar de variáveis discretas, empregado para determinar a importância atribuída a um critério em relação a outro. Permite analisar e classificar múltiplas alternativas utilizando uma função matemática. Se um critério for mais importante diante de outros, terá um peso atribuído maior, em comparação aos demais critérios. A importância relativa de cada critério corresponde ao conceito de compromisso (trade-off).

Segundo o método MAUT, as preferências devem ser modeladas, a fim de se obter uma função utilidade multiatributo. Esta função agrega múltiplas funções de utilidades unidimensionais. Portanto, uma função analítica é obtida para combinar diversos atributos 
(ou critérios). As funções utilidade multiatributo mais usuais são a aditiva e a multilinear. A função utilidade aditiva foi concebida para casos em que há independência aditiva entre os atributos nas preferências, mas pode ser também ser aplicada para casos onde há dependência entre atributos [48].

Considerando as funções de utilidade individuais $U_{l}\left(x_{1}\right), U_{2}\left(x_{2}\right), \ldots, U_{m}\left(x_{m}\right)$ para $m$ diferentes atributos e sendo $p_{1}, p_{2}, \ldots, p_{m}$ os pesos de cada um desses atributos, a função de utilidade aditiva é definida como:

$$
U\left(x_{1}, x_{2}, \ldots x_{m}\right)=p_{1} U_{1}\left(x_{1}\right)+p_{2} U_{2}\left(x_{2}\right)+\ldots+p_{m} U_{m}\left(x_{m}\right)=\sum_{\mathrm{i}} p_{i} U_{i}\left(x_{i}\right)
$$

A função de utilidade aditiva deve ter valores variando de 0 a 1 para cada critério e a soma dos pesos $p_{i}$ deve ser igual a 1 . A nota final de todas as alternativas fica entre 0 e 1 . A escala adotada não precisa ser linear.

Cada forma analítica de função utilidade multiatributo deve ter suas condições de independência avaliadas, a fim de garantir que as preferências estejam em conformidade com os princípios básicos do método MAUT. Assim, no caso do presente estudo, serão utilizadas apenas as métricas $A S P, N C, W S 4$ e $1 / A C$ (esta última métrica será tomada na forma invertida $A C^{-1}$ posto que é a única entre as quatro que decresce com o aumento da vulnerabilidade). Os índices $S$ e $I$, bem como as métricas $A D$ e $D I A$ e $A D$ não serão considerados nas formas analíticas. As duas primeiras, representadas em um plano $S-I$ bidimensional, são difíceis de se adaptar ao método MAUT, que é unidimensional. A métrica $A D$ serviria para medir a perda de nós mas não serve para medir o efeito da retirada de enlaces, já que nesse caso ela não se altera. Já a métrica $D I A$ será preservada para ser utilizada como um indicador de desempenho da rede, muito útil na análise dos resultados obtidos.

Tomando o exemplo da Figura 3.1, retira-se, um por um, cada um dos enlaces calcular os valores de $A S P, N C$, WS4 e 1/A. Por exemplo, retirando o enlace C-D obtém-se o grafo mostrado na Figura 3.2. 


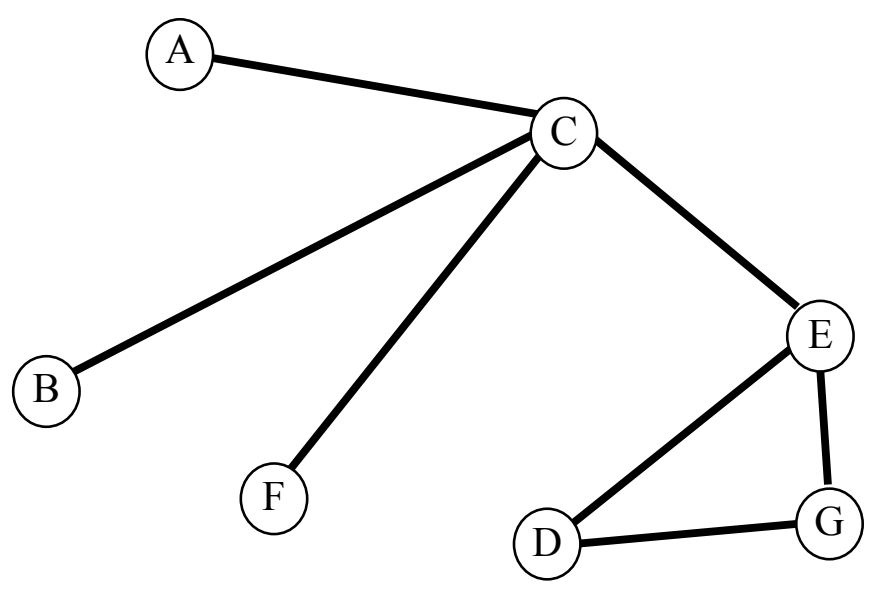

Figura 3.2 - Grafo resultante da Figura 3.1 com a da retirada do enlace CD

Como o grafo da Figura 3.1 é muito simples, é fácil ver o ranking dos enlaces em termos de vulnerabilidade. Os enlaces C-D e C-E são os mais críticos, posto que são os dois enlaces mais centrais, através dos quais passam mais caminhos entre pares de nós. A supressão de qualquer um deles tem igual efeito, pois são simétricos. Na sequência vêm os enlaces E-G e D-G, também simétricos, e finalmente o enlace D-E, que serve apenas para encurtar a distância entre os nós D e E. Não se considera os enlaces A-C, B-C e F-C, tendo em vista que são enlaces que quebram a conectividade da rede quando retirados.

Os valores das métricas obtidos para cada grafo resultante da retirada de um enlace, seguidos dos valores para a rede completa (calculados usando MATLAB) estão mostrados na Tabela 3.12 .

Tabela 3.12 - Valores das métricas com a retirada de enlaces

\begin{tabular}{|ccccccc|}
\hline Métrica & $\begin{array}{c}\text { Retirando } \\
\text { C-D }\end{array}$ & $\begin{array}{c}\text { Retirando } \\
\text { C-E }\end{array}$ & $\begin{array}{c}\text { Retirando } \\
\text { D-E }\end{array}$ & $\begin{array}{c}\text { Retirando } \\
\text { D-G }\end{array}$ & $\begin{array}{c}\text { Retirando } \\
\text { E-G }\end{array}$ & $\begin{array}{c}\text { Rede } \\
\text { completa }\end{array}$ \\
\hline$A S P$ & 0,95 & 0,95 & 0,90 & 0,90 & 0,90 & 0,88 \\
$N C$ & 1,47 & 1,47 & 1,39 & 1,50 & 1,50 & 1,37 \\
$W S 4$ & 2,18 & 2,18 & 2,18 & 1,88 & 1,88 & 1,71 \\
$1 / A C$ & 2,51 & 2,51 & 1,38 & 1,68 & 1,68 & 1,38 \\
\hline
\end{tabular}

Para atribuir os pesos $p_{1}, p_{2}, p_{3}$ e $p_{4}$, para as métricas $A S P, N C, W S 4$ e 1/AC utilizase o método swing weighting [47]. Este método é desenvolvido em quatro passos, conforme explicado a seguir: 
- constrói-se uma tabela com uma alternativa de referência (benchmark, com os valores mínimos dos atributos) e alternativas fictícias nas linhas seguintes, nas quais um atributo por vez assume seu valor máximo;

- define-se um ranking entre as alternativas, arbitrando um fator de preferência entre os atributos;

- atribui-se pontos arbitrariamente para cada atributo, conforme seu ranking, de maneira que o benchmark fique com 0 pontos e a alternativa de ranking 1 fique com 100 pontos;

- calcula-se o peso de cada atributo (número de pontos / total de pontos).

Partindo dos dados da Tabela 3.12 construímos a Tabela 3.13, utilizando o ranking já mencionado, e procede-se à atribuição de pontos conforme o método swing weighting. Como o exemplo da Figura 3.1 aqui adotado é muito simples, a atribuição de pontos pelo decisor não é crítica. Pode ser feita aqui dentro de certo grau de liberdade.

Tabela 3.13 - Atribuição de pesos pelo método swing weighting

\begin{tabular}{|l|cccc|ccc|}
\hline Alternativa fictícia & $\boldsymbol{A S P}$ & $\boldsymbol{N C}$ & $\boldsymbol{W S 4}$ & $\mathbf{1} / \boldsymbol{A C}$ & ranking & pontos & pesos \\
\hline 1. benchmark & 0,88 & 1,37 & 1,71 & 1,38 & 5 & 0 & - \\
2. $A S P$ & $\mathbf{0 , 9 5}$ & 1,37 & 1,71 & 1,38 & 2 & 90 & $31 \%$ \\
3. $N C$ & 0,88 & $\mathbf{1 , 5 0}$ & 1,71 & 1,38 & 3 & 60 & $21 \%$ \\
4. $W S 4$ & 0,88 & 1,37 & $\mathbf{2 , 1 8}$ & 1,38 & 4 & 40 & $14 \%$ \\
5. $1 / A C$ & 0,88 & 1,37 & 1,71 & $\mathbf{2 , 5 1}$ & 1 & 100 & $34 \%$ \\
\hline
\end{tabular}

Como o que aqui importa é analisar o impacto relativo na perda de nós ou enlaces, define-se as funções de utilidade individuais $U_{l}(A S P), U_{2}(N C), U_{3}(W S 4)$ e $U_{4}(1 / A C)$ como sendo a variação de cada métrica em relação ao seu valor de referência (rede com todos os nós e enlaces). Com os valores da Tabela 3.12, omitindo-se as colunas repetidas, chega-se à Tabela 3.14:

Tabela 3.14 - Variações das medidas com a retirada de enlaces

\begin{tabular}{|ccccc|}
\hline Alternativa & $\boldsymbol{\Delta} \boldsymbol{A} \boldsymbol{S P}$ & $\boldsymbol{\Delta} \boldsymbol{N} \boldsymbol{C}$ & $\boldsymbol{\Delta} \boldsymbol{W} \boldsymbol{S} 4$ & $\boldsymbol{\Delta}(\mathbf{1} / \boldsymbol{A C})$ \\
\hline Rede completa & 0,00 & 0,00 & 0,00 & 0,00 \\
Retirando D-E & 0,02 & 0,02 & 0,47 & 0,00 \\
Retirando E-G & 0,02 & 0,14 & 0,17 & 0,30 \\
Retirando C-D & 0,07 & 0,10 & 0,47 & 1,13 \\
\hline
\end{tabular}


Esses valores relativos serão normalizados, assumindo valores entre 0 e 1 (ou de 0 a 100\%), conforme requerido para funções de utilidade. Assim, chega-se à Tabela 3.15:

Tabela 3.15 - Variações das medidas com a retirada de enlaces (0 a 100\%)

\begin{tabular}{|ccccc|}
\hline Alternativa & $\boldsymbol{\Delta} \boldsymbol{A S P}$ & $\boldsymbol{\Delta} \boldsymbol{N C}$ & $\boldsymbol{\Delta} \boldsymbol{W S} 4$ & $\boldsymbol{\Delta}(\mathbf{1} / \boldsymbol{A C})$ \\
\hline Rede completa & $0 \%$ & $0 \%$ & $0 \%$ & $0 \%$ \\
Retirando D-E & $33 \%$ & $18 \%$ & $100 \%$ & $0 \%$ \\
Retirando E-G & $33 \%$ & $100 \%$ & $36 \%$ & $27 \%$ \\
Retirando C-D & $100 \%$ & $75 \%$ & $100 \%$ & $100 \%$ \\
\hline
\end{tabular}

A métrica composta $C$, definida como sendo a função de utilidade aditiva das métricas $A S P, N C, W S 4$ e $1 / A C$, pode ser calculada por meio da Equação 3.12, a partir das funções de utilidade individuais respectivas:

$$
C=U(A S P, N C, W S 4,(1 / A C))=\sum_{\mathrm{i}} p_{i} U_{i}\left(x_{i}\right)
$$

Com os valores funções de utilidade individuais $U i$ da Tabela 3.15 e respectivos pesos individuais $p_{i}$ listados na última coluna da Tabela 3.13, chega-se à Tabela 3.16.

Tabela 3.16 - Funções de utilidade individuais e obtenção da métrica composta $C$

\begin{tabular}{|cccccc|}
\hline Alternativa & $\boldsymbol{p}_{\boldsymbol{l}} \boldsymbol{U}_{\boldsymbol{I}}(\boldsymbol{A S P})$ & $\boldsymbol{p}_{2} \boldsymbol{U}_{2}(\boldsymbol{N C})$ & $\boldsymbol{p}_{3} \boldsymbol{U}_{3}(\boldsymbol{W S} \mathbf{S})$ & $\boldsymbol{p}_{4} \boldsymbol{U}_{4}(\mathbf{1} / \boldsymbol{A C})$ & $\boldsymbol{C}$ \\
\hline Rede completa & $0 \%$ & $0 \%$ & $0 \%$ & $0 \%$ & $0 \%$ \\
Retirando D-E & $9 \%$ & $5 \%$ & $11 \%$ & $0 \%$ & $25 \%$ \\
Retirando E-G & $9 \%$ & $27 \%$ & $4 \%$ & $9 \%$ & $49 \%$ \\
Retirando C-D & $27 \%$ & $20 \%$ & $11 \%$ & $35 \%$ & $93 \%$ \\
\hline
\end{tabular}

O gráfico da Figura 3.3 permite melhor visualizar essas funções de utilidade individuais e a função de utilidade aditiva resultante. Esta última correponde à métrica composta $\boldsymbol{C}$ aqui definida. 


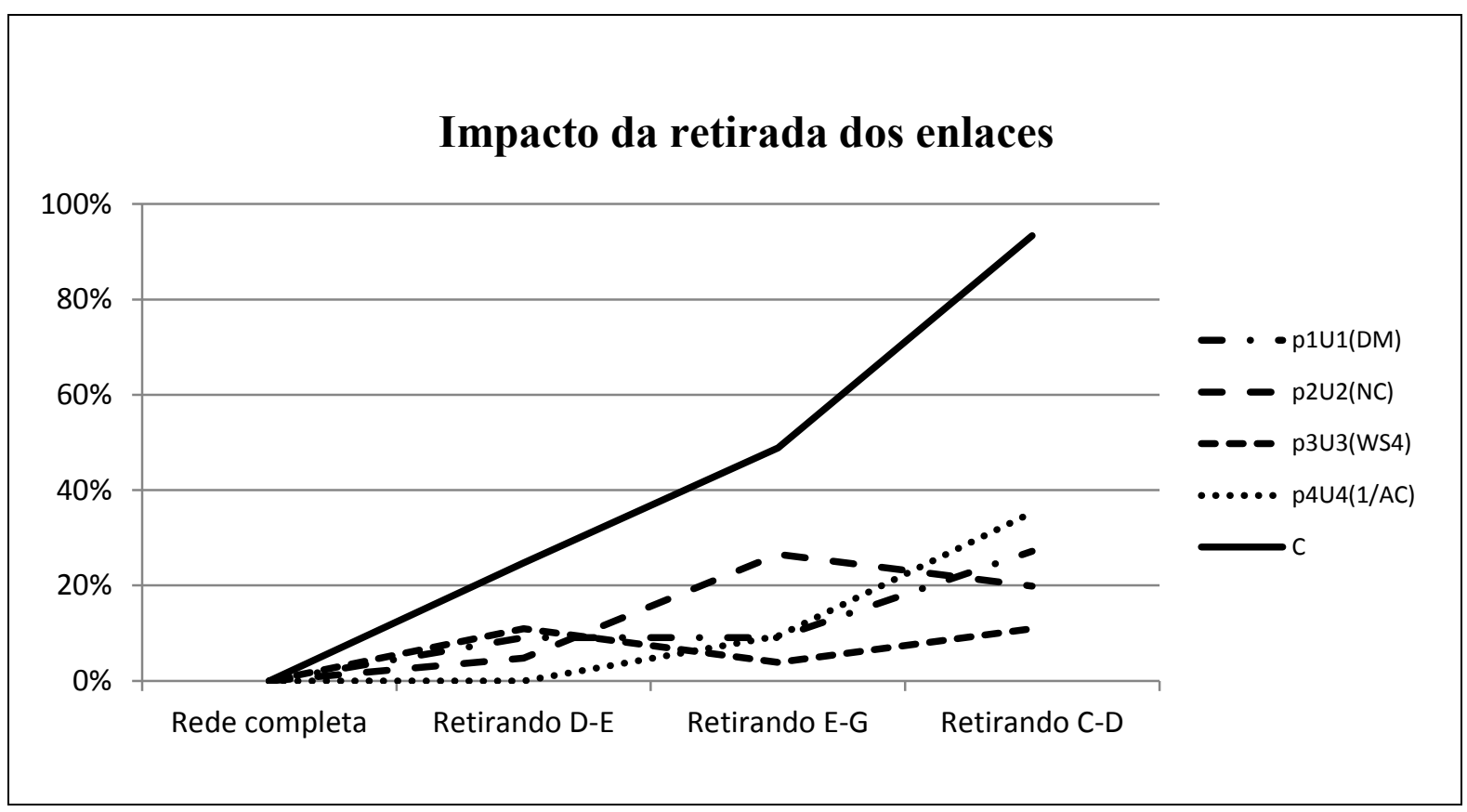

Figura 3.3 - Representação gráfica das métricas individuais e da métrica composta $C$

O gráfico da Figura 3.3 representa o impacto da retirada de enlaces do grafo da Figura 3.1. É possível ver que o impacto indicado pela métrica composta concorda com o ranking que já foi visto. O impacto da retirada do enlace C-D é maior que o de E-G, que por sua vez é maior que o de D-E. Se tomadas individualmente, as métricas $A S P, N C$ e WS4 não concordariam com o ranking, o que só acontece quando são combinadas para formar a métrica composta $C$ aqui proposta

Naturalmente, muitas outras composições das métricas são possíveis. Por exemplo, como o método MAUT não requer que as funções de utilidade individuais sejam lineares, seria possível definí-las em forma logarítmica. Nesse caso, tomados os logaritmos das variações das mesmas métricas $A S P, N C, W S 4$ e $1 / A C$ com pesos iguais, se chegaria a uma métrica composta na forma do logaritmo do produto entre elas.

No presente capítulo foram apresentadas as métricas consideradas neste estudo, bem como sua aplicação a um grafo muito simples (Figura 3.1), visnado apenas explicar como foram calculadas. No próximo capítulo, essas métricas serão aplicadas a grafos de maior complexidade, que representam redes de telecomunicações reais. No entanto, conforme já explicado, apenas quatro entre estas métricas serão utilizadas como funções de utilidade individuais para compor a métrica composta $C$. Para permitir comparações com as mesmas bases, o conjunto de pesos individuais utilizado será o mesmo para todos os experimentos realizados, ajustado com base em simulações exaustivas. 


\section{DESCOBERTA E QUANTIFICAÇÃO DE VULNERABILIDADES}

Simulações e análises foram efetuadas em sete redes distintas, utilizando as oito métricas individuais descritas acima, nomeadamente: o grau médio dos nós $A D$, a distância média $A S P$, os índices $\mathrm{S}$ e I, a criticidade da rede $N C$, a conectividade algébrica $A C$ e o espectro ponderado WS4 (i.e. WS com $\varepsilon=4$ ). A partir das medidas individuais foi calculada a métrica composta $C$ conforme descrita na Seção 3.4.

\subsection{Descrição geral dos experimentos}

Foram realizados experimentos com modelos representando cinco redes brasileiras e duas redes internacionais de porte e topologia comparáveis. Estas últimas são redes bem estudadas em experimentos similares, de maneira que fornecem um boa referência.

No caso das cinco redes que representam operadoras brasileiras, cada nó foi associado a uma cidade ou região metropolitana do Brasil, que estão listadas na Tabela 4.1 em ordem decrescente da população, conforme dados do IBGE [49]. Cada cidade ou região foi associada a um acrônimo ou sigla de duas letras.

Tabela 4.1 - Cidades e regiões metropolitanas

\begin{tabular}{|c|l|r|}
\hline Acrônimo & \multicolumn{1}{|c|}{ Cidade ou Região Metropolitana } & População \\
\hline SP & São Paulo & 20.820 .093 \\
RJ & Rio de Janeiro & 12.064 .657 \\
BH & Belo Horizonte & 4.882 .977 \\
PA & Porto Alegre & 3.979 .561 \\
BS & Brasília & 3.716 .996 \\
RE & Recife & 3.688 .428 \\
FO & Fortaleza & 3.610 .379 \\
SA & Salvador & 3.574 .804 \\
CR & Curitiba & 3.168 .980 \\
CA & Campinas & 3.004 .083 \\
BL & Belém & 2.325 .535 \\
SJ & Vale do Paraíba & 2.258 .956 \\
GO & Goiânia & 2.206 .134 \\
MN & Manaus & 2.175 .860 \\
SO & Sorocaba & 1.726 .785 \\
VI & Vitória & 1.685 .384 \\
SN & Baixada Santista & 1.678 .513 \\
NT & Natal & 1.350 .840 \\
SL & Sao Luís & 1.327 .881 \\
JP & João Pessoa & 1.198 .675 \\
MO & Maceió & 1.156 .278 \\
TE & Teresina & 1.142 .912 \\
JO & Joinville & 1.094 .570 \\
LO & Londrina & 1.043 .518
\end{tabular}




\begin{tabular}{|c|l|r|}
\hline Acrônimo & \multicolumn{1}{|c|}{ Cidade ou Região Metropolitana } & População \\
\hline FL & Florianópolis & 1.012 .831 \\
AJ & Aracaju & 835.654 \\
CB & Cuiabá & 834.060 \\
JF & Juiz de Fora & 786.797 \\
CG & Campo Grande & 786.797 \\
PE & Petrolina & 757.776 \\
RB & Ribeirão Preto & 604.682 \\
UI & Uberlândia & 604.013 \\
CP & Campina Grande & 580.492 \\
FS & Feira de Santana & 556.642 \\
PL & Palmas & 437.996 \\
CX & Caxias do Sul & 435.564 \\
PV & Porto Velho & 428.527 \\
RP & São José do Rio Preto & 408.258 \\
CH & Chapecó & 403.548 \\
JU & Jundiaí & 370.126 \\
MC & Montes Claros & 361.915 \\
IM & Imperatriz & 345.878 \\
BU & Bauru & 343.937 \\
PE & Pelotas & 328.275 \\
PG & Ponta Grossa & 311.611 \\
VC & Vitória da Conquista & 306.866 \\
GV & Governador Valadares & 263.689 \\
SM & Santa Maria & 261.031 \\
FI & Foz do Iguaçu & 256.088 \\
PP & Presidente Prudente & 207.610 \\
ME & Macaé & 206.728 \\
DO & Dourados & 196.035 \\
CS & Criciúma & 192.308 \\
PF & Passo Fundo & 184.826 \\
LA & Lages & 156.727 \\
UR & Uruguaiana & 125.435 \\
VA & Varginha & 123.081 \\
UM & Umuarama & 100.676 \\
IT & Itumbiara & 92.883 \\
PB & Pato Branco & 72.370 \\
\hline & & \\
\hline
\end{tabular}

As redes aqui analisadas são aproximações das redes reais de cinco grandes operadoras brasileiras. São redes que têm backbones de cobertura nacional, pertencentes a uma operadora ou a um conjunto de operadoras de um mesmo grupo econômico. Foram aqui chamadas de Op_A, Op_B, Op_C e Op_D. As redes foram consideradas como independentes e as interconexões entre elas não foram consideradas.

Os dados, apresentados em detalhe no Apêndice A, foram extraídos do Atlas Brasileiro de Telecomunicações de 2014 [50], complementados com informações disponíveis nos sites das próprias operadoras. Alguns detalhes de suas redes não são divulgados de propósito pelas operadoras, por razões comerciais ou de segurança. No 
entanto, os dados publicamente disponíveis são suficientes para o presente estudo. Não foi verificado com as operadoras a exatidão dos dados utilizados, posto que, para fins do presente estudo, basta contar com uma aproximação razoável das redes.

Os grafos que modelam estas redes foram construídos de forma tal que resistem a falhas simples, isto é, a retirada de um único nó ou enlace não quebra a rede em subredes desconectadas. Além de corresponder ao caso usual em backbones, que sempre contam com alguma forma de redundância, esta configuração dos grafos evita incorrer na análise de casos triviais, tais como os que aparecem no exemplo tratado na Seção 3.4.

Conforme explicado em [51], foi disponibilizada uma biblioteca com dados de referência sobre modelos realistas de redes, organizada na forma de uma plataforma para compartilhar dados de planejamento, bem como outras informações exigidas pela comunidade acadêmica. Para contar com referências internacionais entre os experimentos aqui realizados, foram incluídas nas simulações duas redes transcontinentais bem conhecidas. São redes com frequência encontradas em experimentos similares, descritas na biblioteca SNDlib [8], que é uma biblioteca aberta, orientada a projetos de redes fixas de telecomunicações com capacidade de sobrevivência, cuja finalidade é:

- oferecer instâncias de teste para projetos de rede realistas, disponíveis para a comunidade científica;

- servir como um ponto de referência padronizado para testar, avaliar e comparar modelos de projetos de rede e algoritmos;

- $\quad$ ser uma fonte de informações e recursos para projetos de redes fixa; e

- fornecer uma plataforma de contato para pesquisadores e profissionais que trabalham nesta área.

A primeira rede de referência utilizada é a Cost266, a rede de fibra ótica paneuropeia definida no projeto Lion \& Cost action 266, considerando sua topologia ampla (large topology), tal como definida em [52]. Essa rede possui 37 nós e 57 enlaces.

A segunda rede de referência é a Janos US-CA, baseada na rede US NSF incluindo algumas extensões, tal como foi definida na tese de doutorado de János Tapolcai [53]. Essa rede possui 39 nós e 61 enlaces.

Para o caso da rede Op_A, os experimentos realizados serão explicados com maiores detalhes na Seção 4.1. Os experimentos para as demais redes seguem 
procedimentos muito similares, de maneira que seria fastidioso descrevê-los aqui com esse mesmo nível de detalhes. Desta forma, apenas os resultados finais serão apresentados e discutidos nas Seções 4.2 a 4.7. Na Seção 4.8 será apresentada a comparação entre as sete redes e discutidos os resultados globais.

No Apêndice A estão compilados os dados de entrada utilizados em todos os experimentos. Os resultados dos experimentos, com valores detalhados para cada uma das 8 métricas individuais e da métrica composta $C$, são apresentados no Apêndice B.

\subsection{Experimentos Op_A}

\subsubsection{Dados básicos da rede Op_A}

As Tabelas 4.2 e 4.3 contêm todos os dados de entrada da rede Op_A. Estas mesmas tabelas figuram também no Apêndice A.1.

Tabela 4.2 - Dados de entrada da rede Op_A (Nós)

\begin{tabular}{|ccc|}
\hline Nó & $\begin{array}{c}\text { Cidade ou } \\
\text { RM }\end{array}$ & Sigla \\
\hline 1 & São Paulo & SP \\
2 & Rio de Janeiro & RJ \\
3 & Belo Horizonte & BH \\
4 & Porto Alegre & PA \\
5 & Brasília & BS \\
6 & Recife & RE \\
7 & Fortaleza & FO \\
8 & Salvador & SA \\
9 & Curitiba & CR \\
10 & Campinas & CA \\
11 & Belém & BL \\
12 & Goiânia & GO \\
13 & Manaus & MN \\
\hline
\end{tabular}

\begin{tabular}{|ccc|}
\hline Nó & $\begin{array}{c}\text { Cidade ou } \\
\text { RM }\end{array}$ & Sigla \\
\hline 14 & Natal & NT \\
15 & São Luís & SL \\
16 & João Pessoa & JP \\
17 & Maceió & MO \\
18 & Teresina & TE \\
19 & Joinville & JO \\
20 & Florianópolis & FL \\
21 & Aracaju & AJ \\
22 & Cuiabá & CB \\
23 & Juiz de Fora & JF \\
24 & Campo Grande & CG \\
25 & Ribeirão Preto & RB \\
26 & Uberlândia & UI \\
\hline
\end{tabular}

\begin{tabular}{|ccc|}
\hline Nó & $\begin{array}{c}\text { Cidade ou } \\
\text { RM }\end{array}$ & $\begin{array}{c}\text { Sigl } \\
\text { a }\end{array}$ \\
\hline 27 & Palmas & PL \\
28 & Montes Claros & MC \\
29 & Umuarama & UM \\
30 & Ponta Grossa & PG \\
31 & Jundiaí & JU \\
32 & S.J.Rio Preto & RP \\
33 & Chapecó & CH \\
34 & Imperatriz & IM \\
35 & Bauru & BU \\
36 & Santa Maria & SM \\
37 & Pres. Prudente & PP \\
38 & Lages & LA \\
39 & Pato Branco & PB \\
\hline
\end{tabular}

Tabela 4.3 - Dados de entrada da rede Op_A (Enlaces)

\begin{tabular}{|c|c|c|c|c|c|c|c|c|c|}
\hline Enlace & \multirow{2}{*}{$\begin{array}{c}\text { Sigla } \\
\text { SP-RJ }\end{array}$} & Enlace & \multirow{2}{*}{$\frac{\text { Sigla }}{\text { PA-CH }}$} & \multicolumn{2}{|c|}{ Enlace } & \multirow{2}{*}{$\frac{\text { Sigla }}{\text { CR-PG }}$} & \multicolumn{2}{|c|}{ Enlace } & \multirow{2}{*}{$\begin{array}{c}\text { Sigla } \\
\text { JO-FL }\end{array}$} \\
\hline 1 & & 33 & & & 30 & & 19 & & \\
\hline 1 & SP-BH & 36 & PA-SM & 10 & 25 & CA-RB & 22 & 24 & CB-CG \\
\hline 9 & SP-CR & 38 & PA-LA & 10 & 31 & CA-JU & 24 & 29 & CG-UM \\
\hline 30 & SP-PG & 12 & BS-GO & 11 & 13 & BL-MN & 25 & 26 & RB-UI \\
\hline 31 & SP-JU & 27 & BS-PL & 11 & 15 & BL-SL & 25 & 32 & RB-RP \\
\hline 35 & SP-BU & 6 & RE-FO & 11 & 18 & BL-TE & 27 & 34 & PL-IM \\
\hline 2 & RJ-SA & 16 & RE-JP & 11 & 34 & BL-IM & 29 & 30 & UM-PG \\
\hline 223 & RJ-JF & $\begin{array}{ll}6 & 17\end{array}$ & RE-MO & 12 & 22 & GO-CB & 29 & 39 & UM-PB \\
\hline
\end{tabular}




\begin{tabular}{|c|c|c|c|c|c|c|c|c|c|c|c|}
\hline \multicolumn{2}{|c|}{ Enlace } & \multirow{2}{*}{$\begin{array}{c}\text { Sigla } \\
\text { BH-BS }\end{array}$} & \multicolumn{2}{|c|}{ Enlace } & \multirow{2}{*}{$\begin{array}{r}\text { Sigla } \\
\text { FO-SA }\end{array}$} & \multicolumn{2}{|c|}{ Enlace } & \multirow{2}{*}{$\frac{\text { Sigla }}{\text { GO-CG }}$} & \multicolumn{2}{|c|}{ Enlace } & \multirow{2}{*}{$\begin{array}{c}\text { Sigla } \\
\text { PG-LA }\end{array}$} \\
\hline 3 & 5 & & 7 & 8 & & 12 & 24 & & 30 & 38 & \\
\hline 3 & 8 & BH-SA & 7 & 14 & FO-NT & 12 & 26 & GO-UI & 30 & 39 & PG-PB \\
\hline 3 & 26 & BH-UI & 7 & 18 & FO-TE & 13 & 34 & MN-IM & 32 & 37 & RP-PP \\
\hline 3 & 23 & BH-JF & 8 & 21 & SA-AJ & 14 & 16 & NT-JP & 33 & 36 & CH-SM \\
\hline 3 & 28 & BH-MC & 8 & 28 & SA-MC & 15 & 18 & SL-TE & 33 & 38 & CH-LA \\
\hline 4 & 20 & PA-FL & 9 & 19 & CR-JO & 17 & 21 & MO-AJ & 35 & 37 & BU-PP \\
\hline
\end{tabular}

A partir dessas tabelas pode-se facilmente construir as matrizes de adjacência, laplaciana e normal. A partir dessas duas últimas matrizes pode-se obter o espectro laplaciano e o espectro normal, respectivamente. Os procedimentos envolvidos foram explicados no Capítulo 3.

A partir desses dados, os valores para as métricas $A D, D I A, A S P, S, I, A C, N C e$ WS4 foram calculados para a rede Op_A, seguindo os procedimentos explicados em detalhe na Seção 3. Os valores das oito métricas (obtidos com o programa Op_A e as funções MATLAB dos Apêndices C e D) estão na Tabela 4.4.

Tabela 4.4 - Medidas obtidas para a rede Op_A

\begin{tabular}{|c|cccccccc|}
\hline Métrica & $\boldsymbol{A D}$ & $\boldsymbol{D I A}$ & $\boldsymbol{A S P}$ & $\boldsymbol{S}(\mathbf{x 1 0 0 )}$ & $\boldsymbol{I}$ & $\boldsymbol{A C}$ & $\boldsymbol{N C}$ & $\boldsymbol{W S} 4$ \\
\hline Valor & 2,87 & 9 & 2,03 & 0,22 & 0,61 & 0,10 & 1,96 & 8,08 \\
\hline
\end{tabular}

O valor do índice $S$ na Tabela 4.3 foi multiplicado por 100 para deixá-lo visível.

\subsubsection{Retirada de nós e enlaces da rede Op_A}

A partir deste ponto, foram executados dois experimentos sobre a rede Op_A (utilizando o mesmo programa Op_A e funções MATLAB dos Apêndices C e D).

O primeiro experimento consistiu em retirar apenas um nó de cada vez e recalcular as métricas. Assim, essas métricas foram calculadas para o grafo resultante da rede Op_A destituída de seu primeiro nó, em seguida da rede Op_A sem o segundo nó, e assim sucessivamente. Os resultados estão na Tabela 4.5. Os nós retirados em cada rodada são os listados na primeira coluna da Tabela 4.5, seguidos dos valores das medidas obtidas.

Tabela 4.5 - Valores das medidas para a rede Op_A (Retirando-se nós)

\begin{tabular}{|ccccccccc|}
\hline Métrica & $\boldsymbol{A D}$ & $\boldsymbol{D I} \boldsymbol{A}$ & $\boldsymbol{A S P}$ & $\boldsymbol{S}(\mathbf{x 1 0 0})$ & $\boldsymbol{I}$ & $\boldsymbol{A C}$ & $\boldsymbol{N C}$ & $\boldsymbol{W S} 4$ \\
\hline SP & 2,63 & 12 & 2,64 & 1,66 & 0,47 & 0,04 & 3,40 & 9,45 \\
RJ & 2,79 & 9 & 2,06 & 0,13 & 0,60 & 0,09 & 2,08 & 8,03 \\
\hline
\end{tabular}




\begin{tabular}{|c|c|c|c|c|c|c|c|c|}
\hline Métrica & $A D$ & $D I A$ & $A S P$ & $S(\times 100)$ & $I$ & $A C$ & $N C$ & WS4 \\
\hline $\mathrm{BH}$ & 2,63 & 10 & 2,27 & 0,45 & 0,58 & 0,07 & 2,42 & 8,75 \\
\hline PA & 2,74 & 9 & 2,02 & 0,30 & 0,63 & 0,11 & 2,11 & 8,89 \\
\hline $\mathrm{BS}$ & 2,79 & 11 & 2,20 & 0,30 & 0,66 & 0,06 & 2,47 & 8,26 \\
\hline $\mathrm{RE}$ & 2,79 & 9 & 2,03 & 0,32 & 0,62 & 0,10 & 2,08 & 8,36 \\
\hline FO & 2,74 & 11 & 2,28 & 0,30 & 0,60 & 0,06 & 2,85 & 8,80 \\
\hline SA & 2,68 & 14 & 2,61 & 1,44 & 0,64 & 0,03 & 3,38 & 8,83 \\
\hline $\mathrm{CR}$ & 2,79 & 10 & 2,13 & 0,33 & 0,63 & 0,07 & 2,27 & 8,39 \\
\hline CA & 2,84 & 9 & 2,03 & 0,24 & 0,61 & 0,10 & 1,95 & 7,90 \\
\hline $\mathrm{BL}$ & 2,74 & 9 & 2,05 & 0,06 & 0,60 & 0,10 & 2,26 & 9,03 \\
\hline GO & 2,74 & 9 & 2,12 & 0,00 & 0,54 & 0,08 & 2,29 & 8,63 \\
\hline $\mathrm{MN}$ & 2,84 & 9 & 2,00 & 0,28 & 0,63 & 0,11 & 1,89 & 8,11 \\
\hline NT & 2,84 & 9 & 2,02 & 0,29 & 0,61 & 0,11 & 1,93 & 7,87 \\
\hline SL & 2,84 & 9 & 2,01 & 0,25 & 0,63 & 0,11 & 1,90 & 8,06 \\
\hline $\mathrm{JP}$ & 2,84 & 9 & 2,00 & 0,28 & 0,64 & 0,11 & 1,91 & 7,78 \\
\hline MO & 2,84 & 9 & 2,02 & 0,33 & 0,62 & 0,10 & 1,97 & 7,99 \\
\hline $\mathrm{TE}$ & 2,79 & 10 & 2,10 & 0,15 & 0,65 & 0,08 & 2,33 & 8,42 \\
\hline $\mathrm{JO}$ & 2,84 & 9 & 2,05 & 0,25 & 0,59 & 0,09 & 2,06 & 7,87 \\
\hline FL & 2,84 & 9 & 2,01 & 0,31 & 0,63 & 0,10 & 2,00 & 7,98 \\
\hline AJ & 2,84 & 9 & 2,06 & 0,20 & 0,58 & 0,09 & 2,02 & 8,03 \\
\hline $\mathrm{CB}$ & 2,84 & 9 & 2,03 & 0,23 & 0,60 & 0,10 & 1,92 & 8,04 \\
\hline $\mathrm{JF}$ & 2,84 & 9 & 2,05 & 0,07 & 0,59 & 0,10 & 1,93 & 7,93 \\
\hline $\mathrm{CG}$ & 2,79 & 9 & 2,06 & 0,06 & 0,59 & 0,09 & 2,10 & 8,24 \\
\hline $\mathrm{RB}$ & 2,79 & 9 & 2,07 & 0,20 & 0,59 & 0,10 & 2,18 & 8,52 \\
\hline UI & 2,79 & 9 & 2,11 & 0,04 & 0,56 & 0,09 & 2,13 & 8,40 \\
\hline PL & 2,84 & 10 & 2,11 & 0,26 & 0,63 & 0,07 & 2,26 & 8,00 \\
\hline $\mathrm{MC}$ & 2,84 & 9 & 2,05 & 0,10 & 0,60 & 0,10 & 1,94 & 8,11 \\
\hline UM & 2,79 & 9 & 2,08 & 0,01 & 0,58 & 0,09 & 2,11 & 8,16 \\
\hline $\mathrm{PG}$ & 2,68 & 11 & 2,34 & 0,08 & 0,57 & 0,05 & 2,86 & 9,03 \\
\hline JU & 2,84 & 9 & 2,06 & 0,12 & 0,59 & 0,10 & 1,99 & 8,08 \\
\hline $\mathrm{RP}$ & 2,84 & 9 & 2,02 & 0,28 & 0,61 & 0,10 & 1,98 & 8,06 \\
\hline $\mathrm{CH}$ & 2,79 & 9 & 2,01 & 0,25 & 0,63 & 0,11 & 1,93 & 8,25 \\
\hline IM & 2,79 & 10 & 2,07 & 0,37 & 0,68 & 0,08 & 2,22 & 8,27 \\
\hline $\mathrm{BU}$ & 2,84 & 9 & 2,09 & 0,05 & 0,56 & 0,10 & 2,05 & 8,18 \\
\hline SM & 2,84 & 8 & 1,99 & 0,19 & 0,53 & 0,11 & 1,87 & 7,95 \\
\hline PP & 2,84 & 9 & 2,03 & 0,19 & 0,60 & 0,10 & 1,96 & 7,81 \\
\hline LA & 2,79 & 10 & 2,14 & 0,44 & 0,62 & 0,07 & 2,31 & 8,26 \\
\hline PB & 2,84 & 9 & 2,03 & 0,21 & 0,61 & 0,10 & 1,92 & 8,02 \\
\hline
\end{tabular}

O segundo experimento consistiu em retirar apenas um enlace de cada vez e recalcular as mesmas métricas. Assim, de maneira análoga ao primeiro experimento, as métricas foram calculadas para o grafo resultante da rede Op_A destituída de seu primeiro enlace, em seguida da rede Op_A sem o segundo enlace, e assim sucessivamente. 
Os resultados estão na Tabela 4.6. Os enlaces retirados em cada rodada estão listados na primeira coluna, seguido dos valores obtidos para as métricas indicadas.

Tabela 4.6 - Valores das medidas para a rede Op_A (Retirando-se enlaces)

\begin{tabular}{|c|c|c|c|c|c|c|c|c|}
\hline Métrica & $A D$ & $D I A$ & $A S P$ & $S(\mathrm{x100})$ & $I$ & $A C$ & $N C$ & WS4 \\
\hline SP-RJ & 2,85 & 9 & 2,03 & 0,23 & 0,62 & 0,09 & 1,97 & 8,26 \\
\hline SP-BH & 2,85 & 9 & 2,06 & 0,25 & 0,59 & 0,09 & 1,96 & 8,25 \\
\hline SP-CR & 2,85 & 9 & 2,06 & 0,33 & 0,60 & 0,09 & 2,05 & 8,35 \\
\hline SP-PG & 2,85 & 10 & 2,12 & 0,34 & 0,65 & 0,09 & 2,03 & 8,31 \\
\hline SP-JU & 2,85 & 9 & 2,08 & 0,16 & 0,59 & 0,10 & 2,07 & 8,56 \\
\hline SP-BU & 2,85 & 9 & 2,14 & 0,17 & 0,53 & 0,10 & 2,10 & 8,64 \\
\hline RJ-SA & 2,85 & 9 & 2,05 & 0,19 & 0,61 & 0,09 & 1,99 & 8,23 \\
\hline RJ-JF & 2,85 & 9 & 2,03 & 0,23 & 0,62 & 0,10 & 1,98 & 8,05 \\
\hline BH-BS & 2,85 & 9 & 2,07 & 0,15 & 0,59 & 0,10 & 2,00 & 8,31 \\
\hline BH-SA & 2,85 & 9 & 2,05 & 0,21 & 0,61 & 0,09 & 1,99 & 8,25 \\
\hline BH-UI & 2,85 & 9 & 2,08 & 0,07 & 0,58 & 0,10 & 2,00 & 8,28 \\
\hline BH-JF & 2,85 & 9 & 2,04 & 0,22 & 0,61 & 0,10 & 2,00 & 8,20 \\
\hline $\mathrm{BH}-\mathrm{MC}$ & 2,85 & 9 & 2,04 & 0,24 & 0,62 & 0,10 & 2,00 & 8,24 \\
\hline PA-FL & 2,85 & 9 & 2,04 & 0,25 & 0,62 & 0,10 & 1,94 & 8,57 \\
\hline $\mathrm{PA}-\mathrm{CH}$ & 2,85 & 9 & 2,04 & 0,24 & 0,62 & 0,10 & 1,98 & 8,52 \\
\hline PA-SM & 2,85 & 9 & 2,04 & 0,24 & 0,62 & 0,10 & 2,06 & 8,25 \\
\hline PA-LA & 2,85 & 9 & 2,05 & 0,27 & 0,60 & 0,10 & 1,90 & 8,23 \\
\hline BS-GO & 2,85 & 9 & 2,05 & 0,24 & 0,61 & 0,09 & 1,98 & 8,28 \\
\hline BS-PL & 2,85 & 10 & 2,12 & 0,22 & 0,64 & 0,06 & 2,09 & 8,30 \\
\hline RE-FO & 2,85 & 9 & 2,06 & 0,19 & 0,60 & 0,10 & 1,92 & 8,34 \\
\hline RE-JP & 2,85 & 9 & 2,03 & 0,23 & 0,62 & 0,10 & 2,05 & 8,31 \\
\hline RE-MO & 2,85 & 9 & 2,04 & 0,26 & 0,62 & 0,10 & 1,96 & 8,53 \\
\hline FO-SA & 2,85 & 11 & 2,17 & 0,62 & 0,68 & 0,07 & 1,90 & 8,42 \\
\hline FO-NT & 2,85 & 9 & 2,05 & 0,20 & 0,60 & 0,10 & 2,14 & 8,35 \\
\hline FO-TE & 2,85 & 9 & 2,10 & 0,07 & 0,57 & 0,07 & 2,10 & 8,41 \\
\hline SA-AJ & 2,85 & 9 & 2,07 & 0,26 & 0,60 & 0,08 & 2,12 & 8,53 \\
\hline SA-MC & 2,85 & 9 & 2,04 & 0,25 & 0,62 & 0,10 & 1,97 & 8,21 \\
\hline CR-JO & 2,85 & 10 & 2,08 & 0,49 & 0,68 & 0,08 & 2,15 & 8,38 \\
\hline CR-PG & 2,85 & 9 & 2,04 & 0,27 & 0,62 & 0,10 & 1,96 & 8,32 \\
\hline CA-RB & 2,85 & 9 & 2,04 & 0,24 & 0,62 & 0,10 & 1,96 & 8,44 \\
\hline CA-JU & 2,85 & 9 & 2,05 & 0,17 & 0,61 & 0,10 & 1,94 & 8,18 \\
\hline BL-MN & 2,85 & 9 & 2,04 & 0,19 & 0,61 & 0,10 & 2,00 & 8,40 \\
\hline BL-SL & 2,85 & 9 & 2,04 & 0,21 & 0,61 & 0,10 & 2,00 & 8,34 \\
\hline BL-TE & 2,85 & 9 & 2,04 & 0,15 & 0,61 & 0,10 & 1,94 & 8,39 \\
\hline BL-IM & 2,85 & 9 & 2,04 & 0,22 & 0,61 & 0,10 & 1,93 & 8,49 \\
\hline GO-CB & 2,85 & 9 & 2,04 & 0,21 & 0,60 & 0,10 & 2,02 & 8,34 \\
\hline GO-CG & 2,85 & 9 & 2,05 & 0,20 & 0,60 & 0,10 & 1,99 & 8,39 \\
\hline GO-UI & 2,85 & 9 & 2,05 & 0,22 & 0,61 & 0,10 & 1,97 & 8,27 \\
\hline MN-IM & 2,85 & 10 & 2,05 & 0,42 & 0,70 & 0,10 & 1,92 & 8,30 \\
\hline NT-JP & 2,85 & 9 & 2,03 & 0,23 & 0,62 & 0,10 & 2,00 & 8,03 \\
\hline
\end{tabular}




\begin{tabular}{|ccccccccc|}
\hline Métrica & $\boldsymbol{A D}$ & $\boldsymbol{D I} \boldsymbol{A}$ & $\boldsymbol{A S P}$ & $\boldsymbol{S} \mathbf{( x 1 0 0 )}$ & $\boldsymbol{I}$ & $\boldsymbol{A C}$ & $\boldsymbol{N C}$ & $\boldsymbol{W S} \boldsymbol{C}$ \\
\hline SL-TE & 2,85 & 10 & 2,05 & 0,38 & 0,70 & 0,10 & 1,92 & 8,26 \\
MO-AJ & 2,85 & 9 & 2,05 & 0,27 & 0,60 & 0,09 & 1,91 & 8,18 \\
JO-FL & 2,85 & 9 & 2,05 & 0,29 & 0,61 & 0,09 & 2,09 & 8,15 \\
CB-CG & 2,85 & 9 & 2,04 & 0,23 & 0,61 & 0,10 & 1,95 & 8,25 \\
CG-UM & 2,85 & 9 & 2,07 & 0,14 & 0,60 & 0,09 & 2,00 & 8,29 \\
RB-UI & 2,85 & 9 & 2,05 & 0,17 & 0,61 & 0,10 & 1,93 & 8,44 \\
RB-RP & 2,85 & 9 & 2,06 & 0,20 & 0,60 & 0,10 & 2,06 & 8,60 \\
PL-IM & 2,85 & 9 & 2,08 & 0,15 & 0,57 & 0,07 & 2,12 & 8,26 \\
UM-PG & 2,85 & 9 & 2,05 & 0,18 & 0,61 & 0,10 & 1,97 & 8,35 \\
UM-PB & 2,85 & 9 & 2,04 & 0,25 & 0,62 & 0,10 & 2,00 & 8,18 \\
PG-LA & 2,85 & 10 & 2,17 & 0,47 & 0,60 & 0,06 & 2,19 & 8,35 \\
PG-PB & 2,85 & 9 & 2,05 & 0,24 & 0,61 & 0,10 & 2,01 & 8,31 \\
RP-PP & 2,85 & 9 & 2,05 & 0,24 & 0,61 & 0,10 & 1,98 & 8,35 \\
CH-SM & 2,85 & 9 & 2,03 & 0,22 & 0,61 & 0,10 & 2,02 & 8,14 \\
CH-LA & 2,85 & 9 & 2,04 & 0,21 & 0,61 & 0,10 & 1,89 & 8,17 \\
BU-PP & 2,85 & 9 & 2,07 & 0,15 & 0,58 & 0,10 & 2,05 & 8,29 \\
\hline
\end{tabular}

\subsubsection{Métrica composta para a rede Op_A}

Seguindo os procedimentos já explicados na Seção 3.4, pode-se calcular os valores da métrica composta $C$ para os casos dos dois experimentos efetuados com a rede Op_A (retirada de nós e retirada de enlaces).

Para manter a mesma base de comparação, deve-se utilizar os mesmos $p_{i}$ não apenas para a rede Op_A, mas igualmente para as demais redes aqui ensaiadas. Tal como explicado na Seção 3.4, esses pesos podem ser obtidos pelo método swing weighting, que toma por base uma atribuição de pontos, que deve ser escolhida pelo decisor. Na presente seção, o critério para a escolha dos pontos levou em conta adicionalmente o objetivo de se equilibrar os impactos das quatro métricas selecionadas, com base nos resultados obtidos em todos os experimentos feitos para as sete redes, que produziram mais de 5000 medidas individuais.

O procedimento adotado foi partir das medidas individuais, que estão tabuladas no Apêndice B. A partir dessas medidas foram construídas tabelas similares à Tabela 3.15, contendo as variações ponderadas das quatro métricas $\left(p_{1} \Delta A S P, p_{2} \Delta N C, p_{3} \Delta W S 4 \mathrm{e}\right.$ $p_{4} \Delta(1 / A C)$, deixando $p_{1}, p_{2}, p_{3}$ e $p_{4}$ como variáveis. Iniciando-se com pesos iguais, $\left(p_{1}=p_{2}\right.$ $=p_{3}=p_{4}=25 \%$ ), os pesos foram variados incrementalmente, para mais ou para menos, e calculados os desvios padrões para cada coluna de cada tabela (similares à Tabela 3.15). A 
variação incremental dos pesos foi reiterada até que a soma dos desvios padrões médios de cada tabela chegasse ao seu valor mínimo (simulações feitas com MS-Excel).

Os pesos assim obtidos foram aplicados na Equação 3.13, o que resultou na seguinte expressão, que será utilizada para o cálculo da métrica composta em todos os experimentos de todas as redes, tanto para retirada de nós quanto para enlaces:

$$
C=\sum_{\mathrm{i}} p_{i} U_{i}\left(x_{i}\right)=0,27 \Delta A S P+0,27 \Delta N C+0,11 \Delta W S 4+0,35 \Delta(1 / A C)
$$

Deve-se lembrar que os valores das funções de utilidade individuais $U i$ foram definidos como sendo a variação de cada medida com relação ao seu valor de referência e que os valores de $U i$ são normalizados, isto é, são expressos em uma escala 0 a $100 \%$.

Calculando os termos da Equação 4.1 a partir dos valores de referência da Tabela 4.4 (valores para a rede Op_A completa) e das medidas da Tabela 4.5 (retirada de nós da rede Op_A), pode-se obter os valores correspondentes para a métrica $C$. Para facilitar a leitura e sua representação em forma de gráfico, os nós foram reordenados em ordem decrescente dos valores de $C$. Os resultados estão na Tabela 4.7.

Tabela 4.7 - Métrica composta $C$ para a retirada de nós da rede Op_A

\begin{tabular}{|cccccc|}
\hline Nó retirado & $\mathbf{0 , 2 7} \boldsymbol{\Delta A S P}$ & $\mathbf{0 , 2 7} \boldsymbol{\Delta N C}$ & $\mathbf{0 , 1 1} \boldsymbol{\Delta} \boldsymbol{W S} \mathbf{4}$ & $\mathbf{0 , 3 5} \boldsymbol{\Delta}(\mathbf{1} / \boldsymbol{A C})$ & $\boldsymbol{C}$ \\
\hline SA & $26 \%$ & $27 \%$ & $7 \%$ & $35 \%$ & $95 \%$ \\
SP & $27 \%$ & $27 \%$ & $11 \%$ & $23 \%$ & $88 \%$ \\
PG & $15 \%$ & $18 \%$ & $8 \%$ & $16 \%$ & $56 \%$ \\
FO & $12 \%$ & $17 \%$ & $7 \%$ & $10 \%$ & $46 \%$ \\
BH & $12 \%$ & $10 \%$ & $6 \%$ & $7 \%$ & $35 \%$ \\
BS & $9 \%$ & $11 \%$ & $3 \%$ & $12 \%$ & $34 \%$ \\
LA & $6 \%$ & $8 \%$ & $3 \%$ & $8 \%$ & $25 \%$ \\
GO & $5 \%$ & $7 \%$ & $6 \%$ & $6 \%$ & $24 \%$ \\
CR & $6 \%$ & $7 \%$ & $4 \%$ & $7 \%$ & $24 \%$ \\
TE & $5 \%$ & $8 \%$ & $4 \%$ & $5 \%$ & $22 \%$ \\
PL & $5 \%$ & $7 \%$ & $1 \%$ & $8 \%$ & $22 \%$ \\
BL & $3 \%$ & $7 \%$ & $8 \%$ & $2 \%$ & $19 \%$ \\
IM & $3 \%$ & $6 \%$ & $3 \%$ & $6 \%$ & $18 \%$ \\
UI & $5 \%$ & $5 \%$ & $4 \%$ & $2 \%$ & $16 \%$ \\
RB & $3 \%$ & $5 \%$ & $5 \%$ & $2 \%$ & $15 \%$ \\
CG & $3 \%$ & $4 \%$ & $3 \%$ & $3 \%$ & $14 \%$ \\
PA & $1 \%$ & $4 \%$ & $7 \%$ & $0 \%$ & $13 \%$ \\
UM & $4 \%$ & $4 \%$ & $2 \%$ & $3 \%$ & $13 \%$ \\
RJ & $3 \%$ & $4 \%$ & $2 \%$ & $4 \%$ & $12 \%$ \\
BU & $4 \%$ & $3 \%$ & $3 \%$ & $1 \%$ & $11 \%$ \\
\hline
\end{tabular}




\begin{tabular}{|cccccc|}
\hline Nó retirado & $\mathbf{0 , 2 7} \mathbf{\Delta} \boldsymbol{A S P}$ & $\mathbf{0 , 2 7} \boldsymbol{\Delta} \boldsymbol{N C}$ & $\mathbf{0 , 1 1} \boldsymbol{\Delta} \boldsymbol{W S} \mathbf{4}$ & $\mathbf{0 , 3 5} \boldsymbol{\Delta ( \mathbf { 1 } / \boldsymbol { A C } )}$ & $\boldsymbol{C}$ \\
\hline RE & $2 \%$ & $4 \%$ & $4 \%$ & $1 \%$ & $10 \%$ \\
AJ & $3 \%$ & $3 \%$ & $2 \%$ & $2 \%$ & $10 \%$ \\
JO & $3 \%$ & $3 \%$ & $1 \%$ & $3 \%$ & $9 \%$ \\
JU & $3 \%$ & $2 \%$ & $2 \%$ & $1 \%$ & $8 \%$ \\
MC & $3 \%$ & $1 \%$ & $2 \%$ & $1 \%$ & $7 \%$ \\
RP & $2 \%$ & $2 \%$ & $2 \%$ & $1 \%$ & $6 \%$ \\
MO & $1 \%$ & $2 \%$ & $1 \%$ & $1 \%$ & $6 \%$ \\
FL & $1 \%$ & $2 \%$ & $1 \%$ & $1 \%$ & $6 \%$ \\
JF & $2 \%$ & $1 \%$ & $1 \%$ & $1 \%$ & $5 \%$ \\
CB & $2 \%$ & $1 \%$ & $2 \%$ & $1 \%$ & $5 \%$ \\
CH & $1 \%$ & $1 \%$ & $3 \%$ & $0 \%$ & $5 \%$ \\
PB & $2 \%$ & $1 \%$ & $2 \%$ & $1 \%$ & $5 \%$ \\
CA & $2 \%$ & $1 \%$ & $1 \%$ & $1 \%$ & $5 \%$ \\
PP & $2 \%$ & $2 \%$ & $0 \%$ & $1 \%$ & $5 \%$ \\
MN & $1 \%$ & $0 \%$ & $2 \%$ & $0 \%$ & $4 \%$ \\
NT & $1 \%$ & $1 \%$ & $1 \%$ & $0 \%$ & $3 \%$ \\
SL & $1 \%$ & $0 \%$ & $2 \%$ & $0 \%$ & $3 \%$ \\
JP & $0 \%$ & $1 \%$ & $0 \%$ & $0 \%$ & $1 \%$ \\
SM & $0 \%$ & $0 \%$ & $1 \%$ & $0 \%$ & $1 \%$ \\
Rede completa & $0 \%$ & $0 \%$ & $0 \%$ & $0 \%$ & $0 \%$ \\
\hline
\end{tabular}

O gráfico da Figura 4.1 permite melhor visualizar o cálculo da métrica composta para o caso de retirada de nós da rede Op_A.

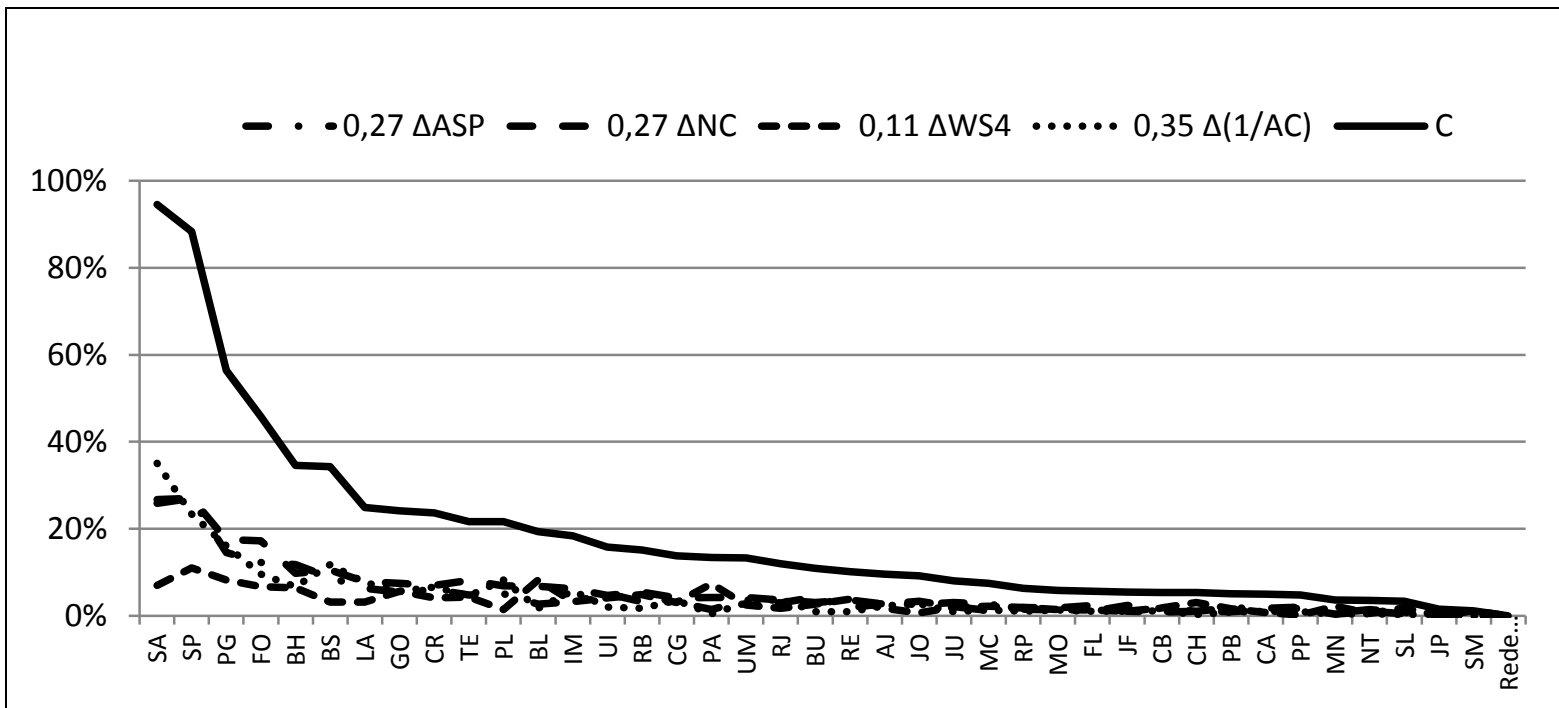

Figura 4.1 - Variações individuais e métrica $C$ na retirada de nós da rede Op_A

De maneira similar, calculando os termos da Equação 4.1 a partir dos valores de referência da Tabela 4.4 (valores para a rede completa) e das medidas da Tabela 4.6 (retirada de enlaces), pode-se obter os valores correspondentes para a métrica $C$ para o 
experimento de retirada de enlaces da rede Op_A. Para maior clareza, os enlaces foram reordenados em ordem decrescente dos valores de $C$. Os resultados estão na Tabela 4.8.

Tabela 4.8 - Métrica composta $C$ para a retirada de enlaces da rede Op_A

\begin{tabular}{|c|c|c|c|c|c|}
\hline Enlace retirado & $0,27 \triangle A S P$ & $0,27 \Delta N C$ & $0,11 \Delta W S 4$ & $0,35 \Delta(1 / A C)$ & C \\
\hline PG-LA & $7 \%$ & $6 \%$ & $4 \%$ & $12 \%$ & $29 \%$ \\
\hline BS-PL & $6 \%$ & $4 \%$ & $3 \%$ & $11 \%$ & $24 \%$ \\
\hline FO-TE & $5 \%$ & $4 \%$ & $4 \%$ & $8 \%$ & $21 \%$ \\
\hline PL-IM & $4 \%$ & $4 \%$ & $3 \%$ & $8 \%$ & $20 \%$ \\
\hline FO-SA & $8 \%$ & $0 \%$ & $4 \%$ & $7 \%$ & $19 \%$ \\
\hline CR-JO & $4 \%$ & $5 \%$ & $4 \%$ & $6 \%$ & $18 \%$ \\
\hline SP-BU & $6 \%$ & $4 \%$ & $6 \%$ & $1 \%$ & $17 \%$ \\
\hline SA-AJ & $3 \%$ & $4 \%$ & $5 \%$ & $4 \%$ & $17 \%$ \\
\hline SP-PG & $6 \%$ & $3 \%$ & $4 \%$ & $3 \%$ & $15 \%$ \\
\hline SP-JU & $4 \%$ & $4 \%$ & $5 \%$ & $1 \%$ & $14 \%$ \\
\hline SP-CR & $3 \%$ & $3 \%$ & $4 \%$ & $4 \%$ & $13 \%$ \\
\hline RB-RP & $3 \%$ & $3 \%$ & $5 \%$ & $1 \%$ & $13 \%$ \\
\hline CG-UM & $3 \%$ & $2 \%$ & $3 \%$ & $4 \%$ & $12 \%$ \\
\hline FO-NT & $3 \%$ & $5 \%$ & $4 \%$ & $2 \%$ & $12 \%$ \\
\hline JO-FL & $3 \%$ & $4 \%$ & $2 \%$ & $3 \%$ & $12 \%$ \\
\hline SP-BH & $3 \%$ & $2 \%$ & $3 \%$ & $3 \%$ & $11 \%$ \\
\hline BU-PP & $4 \%$ & $3 \%$ & $3 \%$ & $1 \%$ & $11 \%$ \\
\hline BS-GO & $2 \%$ & $2 \%$ & $3 \%$ & $3 \%$ & $11 \%$ \\
\hline BH-UI & $4 \%$ & $2 \%$ & $3 \%$ & $2 \%$ & $11 \%$ \\
\hline RJ-SA & $2 \%$ & $2 \%$ & $3 \%$ & $3 \%$ & $11 \%$ \\
\hline GO-CG & $3 \%$ & $2 \%$ & $4 \%$ & $2 \%$ & $10 \%$ \\
\hline BH-BS & $3 \%$ & $2 \%$ & $3 \%$ & $1 \%$ & $10 \%$ \\
\hline SP-RJ & $2 \%$ & $2 \%$ & $3 \%$ & $3 \%$ & $10 \%$ \\
\hline RE-MO & $2 \%$ & $2 \%$ & $5 \%$ & $1 \%$ & $10 \%$ \\
\hline GO-CB & $2 \%$ & $3 \%$ & $4 \%$ & $1 \%$ & $10 \%$ \\
\hline BH-SA & $2 \%$ & $2 \%$ & $3 \%$ & $2 \%$ & $10 \%$ \\
\hline RE-JP & $2 \%$ & $3 \%$ & $3 \%$ & $1 \%$ & $10 \%$ \\
\hline $\mathrm{PA}-\mathrm{CH}$ & $2 \%$ & $2 \%$ & $5 \%$ & $1 \%$ & $10 \%$ \\
\hline RB-UI & $2 \%$ & $1 \%$ & $4 \%$ & $2 \%$ & $10 \%$ \\
\hline PA-FL & $2 \%$ & $1 \%$ & $5 \%$ & $1 \%$ & $10 \%$ \\
\hline PG-PB & $3 \%$ & $2 \%$ & $4 \%$ & $1 \%$ & $10 \%$ \\
\hline PA-SM & $2 \%$ & $3 \%$ & $3 \%$ & $1 \%$ & $9 \%$ \\
\hline BL-MN & $2 \%$ & $2 \%$ & $4 \%$ & $1 \%$ & $9 \%$ \\
\hline BL-IM & $2 \%$ & $1 \%$ & $5 \%$ & $1 \%$ & $9 \%$ \\
\hline CA-RB & $2 \%$ & $2 \%$ & $4 \%$ & $1 \%$ & $9 \%$ \\
\hline UM-PG & $2 \%$ & $2 \%$ & $4 \%$ & $1 \%$ & $9 \%$ \\
\hline BH-MC & $2 \%$ & $2 \%$ & $3 \%$ & $1 \%$ & $9 \%$ \\
\hline RP-PP & $2 \%$ & $2 \%$ & $4 \%$ & $1 \%$ & $9 \%$ \\
\hline BL-SL & $2 \%$ & $2 \%$ & $4 \%$ & $1 \%$ & $9 \%$ \\
\hline RE-FO & $3 \%$ & $1 \%$ & $4 \%$ & $1 \%$ & $9 \%$ \\
\hline
\end{tabular}




\begin{tabular}{|cccccc|}
\hline Enlace retirado & $\mathbf{0 , 2 7} \boldsymbol{\Delta} \boldsymbol{A S P}$ & $\mathbf{0 , 2 7} \boldsymbol{\Delta N C}$ & $\mathbf{0 , 1 1} \boldsymbol{\Delta} \boldsymbol{W S} \mathbf{4}$ & $\mathbf{0 , 3 5} \boldsymbol{\Delta ( 1 / A C )}$ & $\boldsymbol{C}$ \\
\hline BL-TE & $2 \%$ & $1 \%$ & $4 \%$ & $1 \%$ & $8 \%$ \\
CR-PG & $2 \%$ & $2 \%$ & $4 \%$ & $1 \%$ & $8 \%$ \\
GO-UI & $2 \%$ & $2 \%$ & $3 \%$ & $1 \%$ & $8 \%$ \\
MO-AJ & $3 \%$ & $1 \%$ & $3 \%$ & $2 \%$ & $8 \%$ \\
BH-JF & $2 \%$ & $2 \%$ & $3 \%$ & $1 \%$ & $8 \%$ \\
SA-MC & $2 \%$ & $2 \%$ & $3 \%$ & $1 \%$ & $8 \%$ \\
CH-SM & $2 \%$ & $3 \%$ & $2 \%$ & $1 \%$ & $8 \%$ \\
UM-PB & $2 \%$ & $2 \%$ & $3 \%$ & $1 \%$ & $8 \%$ \\
MN-IM & $2 \%$ & $1 \%$ & $3 \%$ & $1 \%$ & $8 \%$ \\
CB-CG & $2 \%$ & $1 \%$ & $3 \%$ & $1 \%$ & $8 \%$ \\
PA-LA & $3 \%$ & $0 \%$ & $3 \%$ & $1 \%$ & $8 \%$ \\
SL-TE & $2 \%$ & $1 \%$ & $3 \%$ & $1 \%$ & $8 \%$ \\
CA-JU & $2 \%$ & $1 \%$ & $3 \%$ & $1 \%$ & $7 \%$ \\
NT-JP & $2 \%$ & $2 \%$ & $2 \%$ & $1 \%$ & $7 \%$ \\
RJ-JF & $2 \%$ & $2 \%$ & $2 \%$ & $1 \%$ & $7 \%$ \\
CH-LA & $2 \%$ & $0 \%$ & $3 \%$ & $1 \%$ & $6 \%$ \\
Rede completa & $0 \%$ & $0 \%$ & $0 \%$ & $0 \%$ & $0 \%$ \\
\hline
\end{tabular}

O gráfico da Figura 4.2 permite melhor visualizar o cálculo da métrica composta $C$ para o caso de retirada de enlaces da rede Op_A.

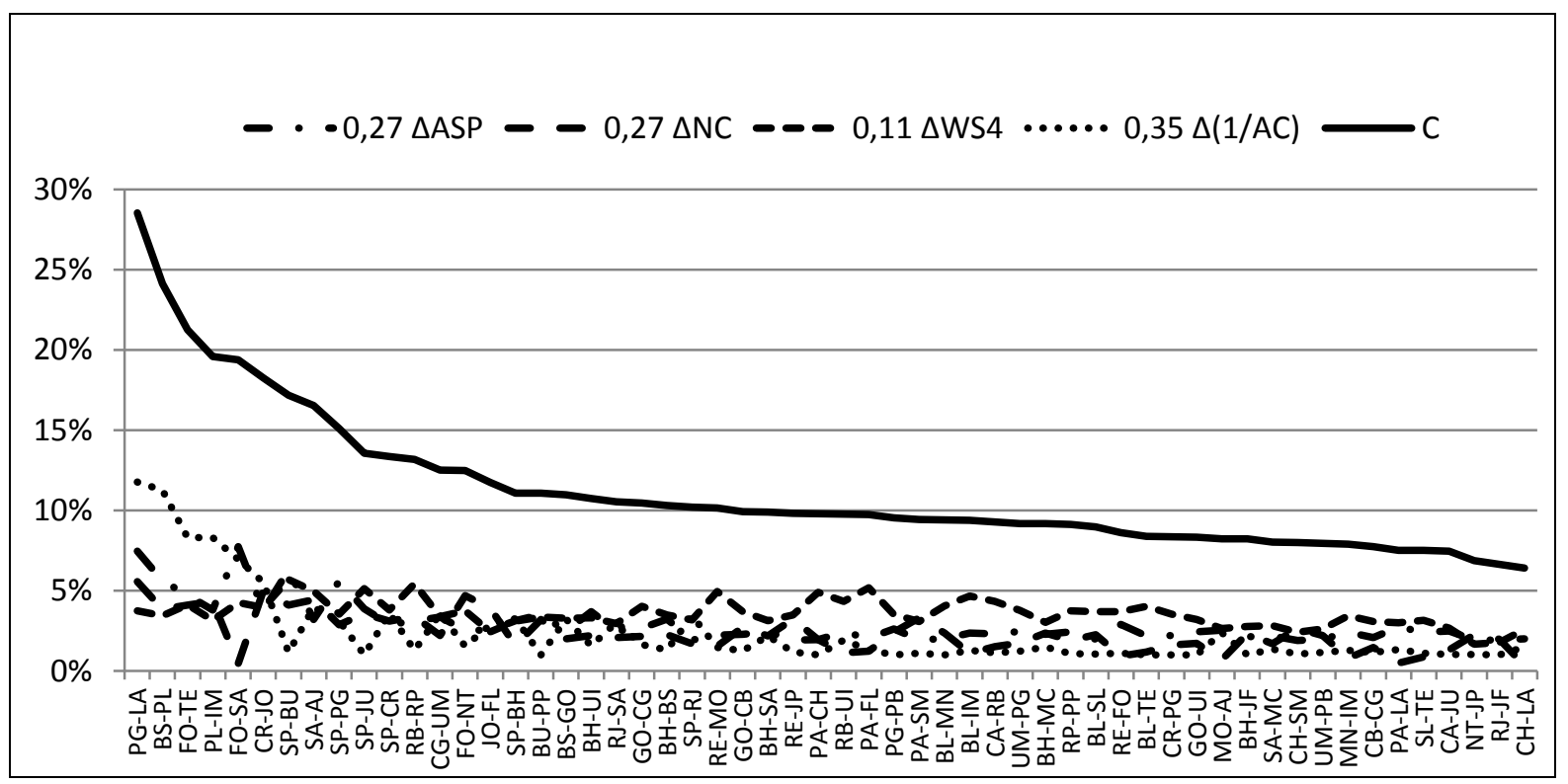

Figura 4.2 - Variações individuais e métrica $C$ na retirada de enlaces da rede Op_A

\subsubsection{Nós e enlaces mais críticos da rede Op_A}

Os nós mais críticos para a rede Op_A, tomando como base a métrica composta $C$, estão destacados na Tabela 4.7 e mostrados no gráfico da Figura 4.1. De maneira similar, os enlaces mais críticos para a rede Op_A podem ser observados na Tabela 4.8 e no gráfico 
da Figura 4.2. Os dois nós e os dois enlaces mais críticos para a rede Op_A estão representados no mapa da Figura 4.3.

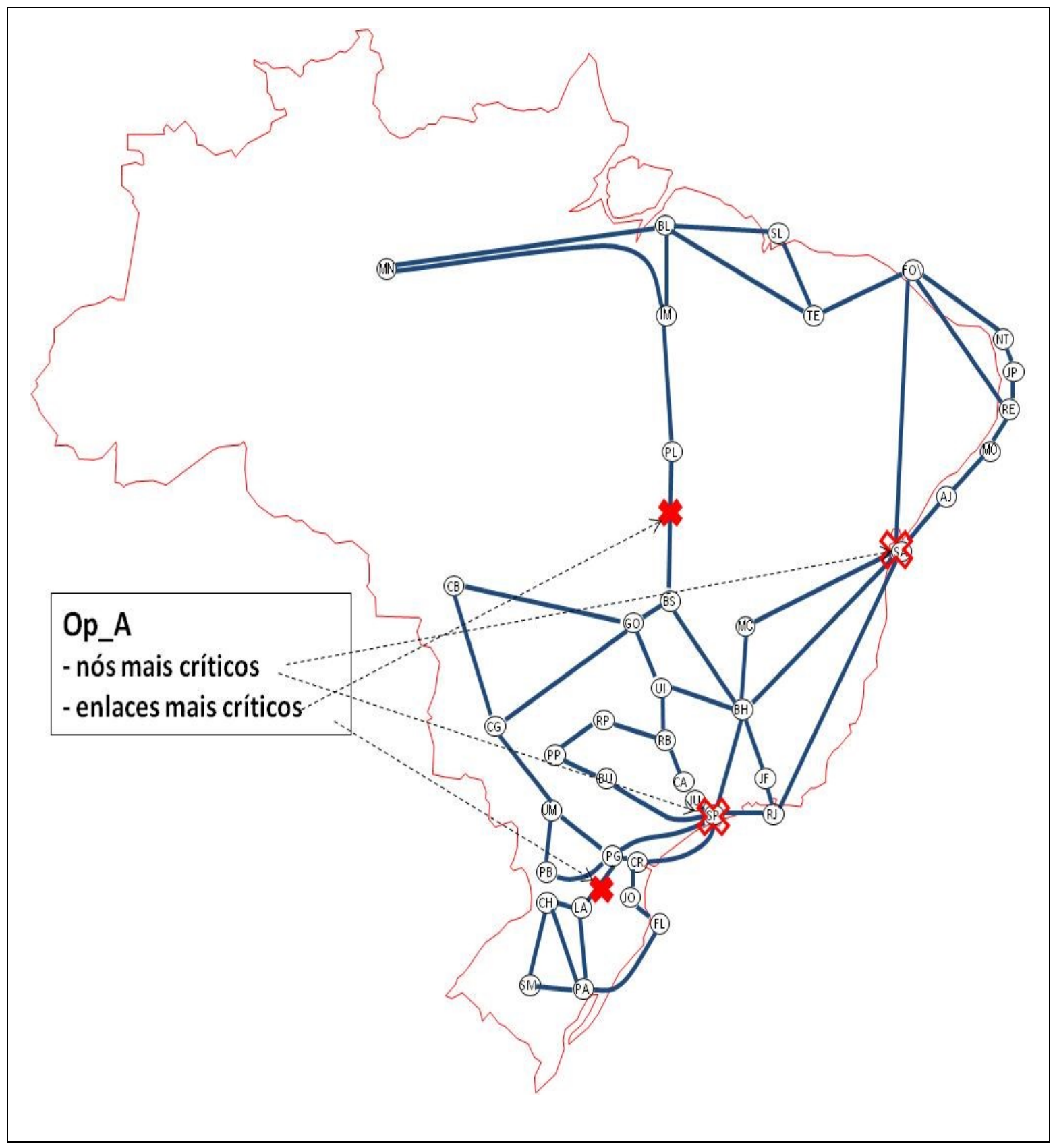

Figura 4.3 - Nós e enlaces mais críticos da rede Op_A

Os nós mais críticos são as regiões metropolitanas de SA (Salvador-BA) e SP (São Paulo-SP). Não é difícil visualizar no mapa da Figura 4.3 que a perda do nó SA tem forte impacto na conectividade de toda a região Nordeste do Brasil. Por exemplo, comparando o valor da métrica DIA da Tabela 4.4 observando o valor que figura na linha correspondente à retirada do nó SA na Tabela 4.5, é possível ver que o diâmetro da rede subiria de 9 para 14 nesse caso. A supressão do nó SP, de forma análoga, teria forte impacto na 
conectividade da região Sul do Brasil. Nesse último caso, pode-se ver na Tabela 4.5 que o diâmetro da rede passaria de 9 para 12 .

Vê-se assim que a métrica composta $C$ é consistente com a observação visual do mapa e com um indicador de desempenho da rede, como nos casos de variação de seu diâmetro. Analisando os dados da Tabela 4.7 pode-se ainda observar que há boa concordância entre métricas individuais e a métrica $C$. Pelas contribuições das métricas $A S P$ e $N C$ os nós SA e SP estão virtualmente empatados. A variação da métrica WS4 favorece ligeiramente ao nó SP, enquanto a variação de $1 / C A$ favorece ao nó $\mathrm{SA}$, mas a variação de WS4 para esse nó ainda é bastante expressiva.

De forma similar, os enlaces mais críticos da rede Op_A são PG-LA (Ponta Grossa-PR a Lages-SC) e BS-PL (Brasília-DF a Palmas-TO), conforme mostrado na Figura 4.3. No mapa é fácil visualizar que a perda de conectividade resultante da retirada desses enlaces é forte, é não é fácil dizer qual dos dois é o mais crítico. O valor da métrica $C$ na Tabela 4.8 indica que o enlace PG-LA é o mais crítico e que do segundo lugar em adiante há vários outros enlaces quase tão críticos quanto BSPL.

Uma forma mais cômoda para se identificar os nós e os enlaces mais críticos é redesenhar os gráficos das Figuras 4.1 e 4.2 de maneira a ressaltar vales e picos. Com esta finalidade os mesmos gráficos são apresentados com os nós e enlaces na sua ordem original, tal qual foram listados nas Tabelas 4.1 e 4.2 .

Assim, as informações sobre os nós mais críticos da rede Op_A aparecem destacadas em picos, que ficam bem visíveis gráfico da Figura 4.4.

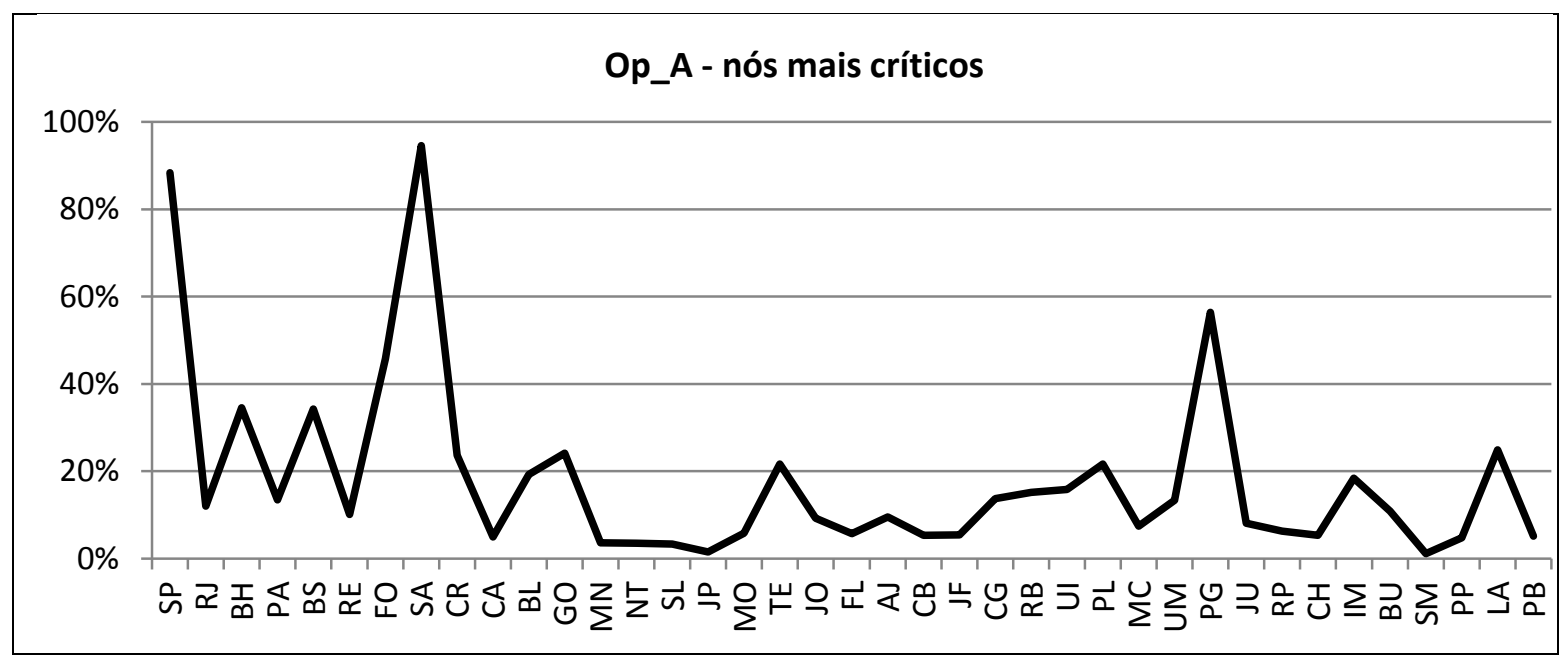

Figura 4.4 - Op_A - resultados obtidos com retirada de nós 
Os picos no gráfico da Figura 4.4 deixam evidente que os dois nós mais críticos são SA e SP, praticamente empatados, e o nó PG (Ponta Grossa-PA) bem abaixo, em terceiro lugar. A partir do quarto lugar os picos são bem modestos, indicando que os demais nós da rede Op_A são relativamente pouco críticos.

De maneira similar, as informações sobre os enlaces mais críticos da rede Op_A são fáceis de se observar nos picos que aparecem no gráfico da Figura 4.5.

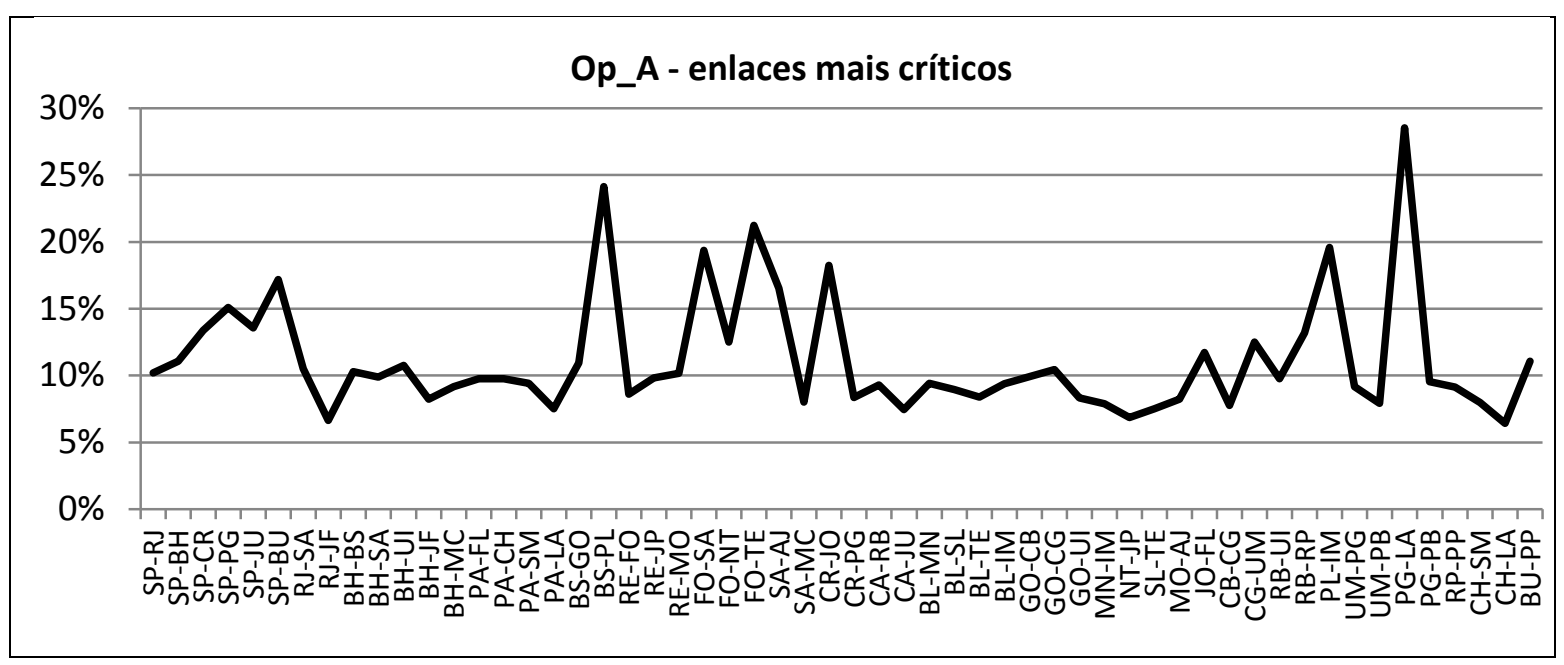

Figura 4.5 - Op_A - resultados obtidos com retirada de enlaces

Observa-se que o gráfico tem uma escala vertical distinta, pois obviamente, dentro de uma mesma rede, a retirada do enlace mais crítico sempre terá um impacto inferior ao da perda do nó mais crítico. A supressão de um leva à perda de todos os seus enlaces. Assim, identificado o enlace mais crítico, a perda de qualquer nó a ele conectado terá sempre um impacto igual ou superior ao da retirada desse enlace. Naturalmente, a perda do enlace mais crítico pode ter impacto superior ao da perda de outros nós da mesma rede, como ocorre aqui com a maioria dos nós considerados não críticos da rede Op_A.

No gráfico da Figura 4.5 é fácil observar não apenas que o enlace PG-LA é o mais crítico, mas também que há pelo menos outros seis enlaces que podem ser considerados como relativamente críticos na rede Op_A. São eles: BS-PL (Brasília-DF a Palmas-TO), FO-TE (Fortaleza-CE a Teresina-PI), FO-SA (Fortaleza-CE a Salvador-BA), SP-BU (São Paulo-SP a Bauru-SP) e PL-IM (Palmas-TO a Imperatriz-MA).

Em resumo, a rede Op_A possui dois nós muito vulneráveis, SA e SP, que podem ser apontados como seus "pontos críticos" ou seus "conectores globais". Os demais nós e todos os enlaces dessa rede são bastante menos vulneráveis. Além disso, não se observa nenhuma concentração geográfica preocupante desses pontos. 


\subsection{Experimentos Op_B}

O modelo utilizado para a rede Op_B está representado na Figura 4.6, na qual estão indicados os dois nós e o enlace mais críticos, conforme se explica mais abaixo.

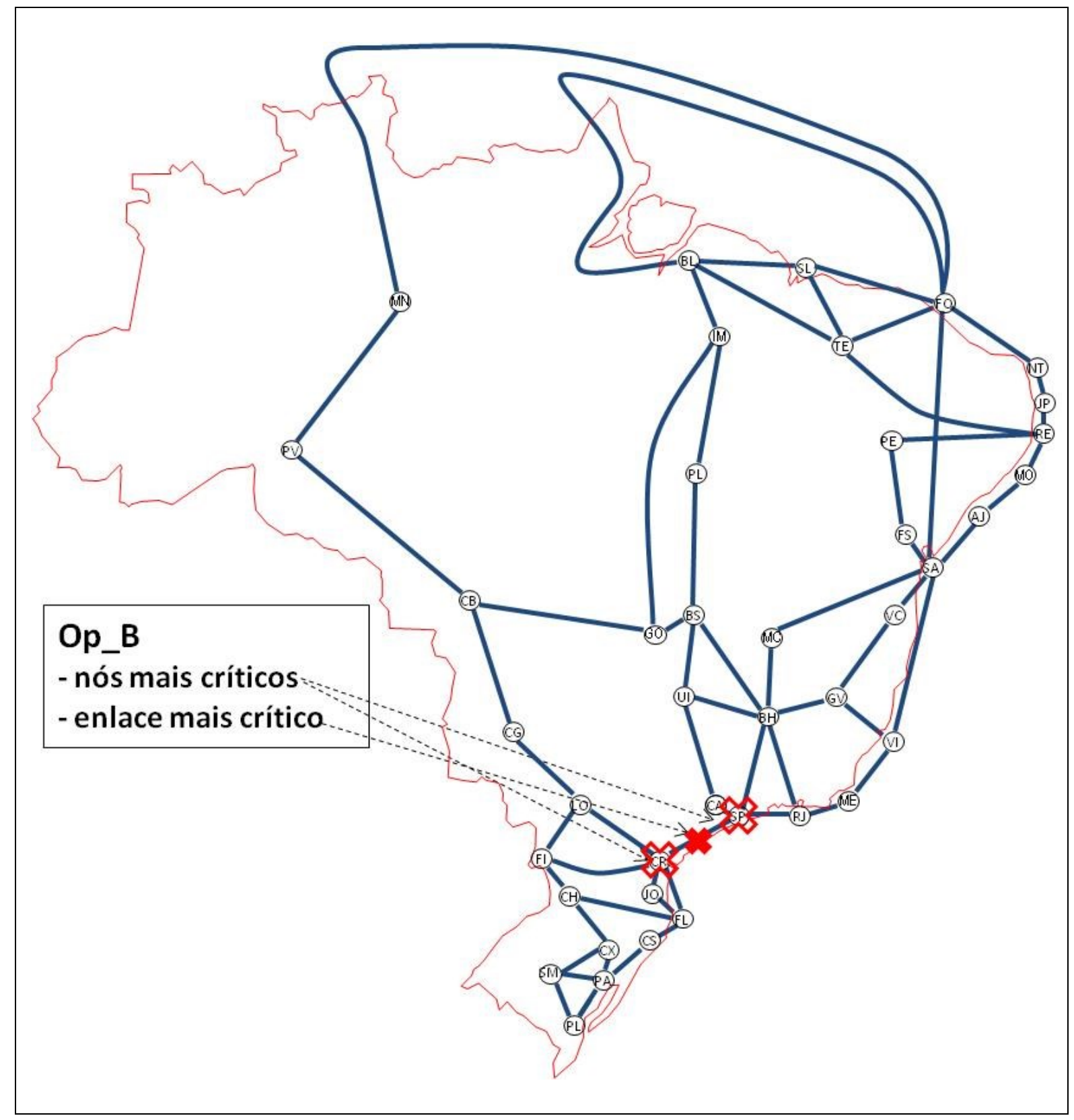

Figura 4.6 - Nós e enlaces mais críticos da rede Op_B

Nesse caso, os nós mais críticos são CR (Curitiba-PR) e SP (São Paulo-SP), que correspondem aos dois maiores picos gráficos da Figura 4.7. É fácil ver no mapa que a degradação na conectividade da rede com a retirada de qualquer desses nós é enorme. Em ambos os casos, o diâmetro da rede passa de 10 para 13 e todas as métricas individuais refletem essa vulnerabilidade. No caso de WS4 a variação também é expressiva, embora essa métrica aponte dois outros nós, FO (Fortaleza-CE) e SA (Salvador-BA), como mais 
críticos. Isso se explica porque WS4 reflete apenas a variação no número de caminhos disjuntos, que seria ligeiramente maior para esses dois nós.

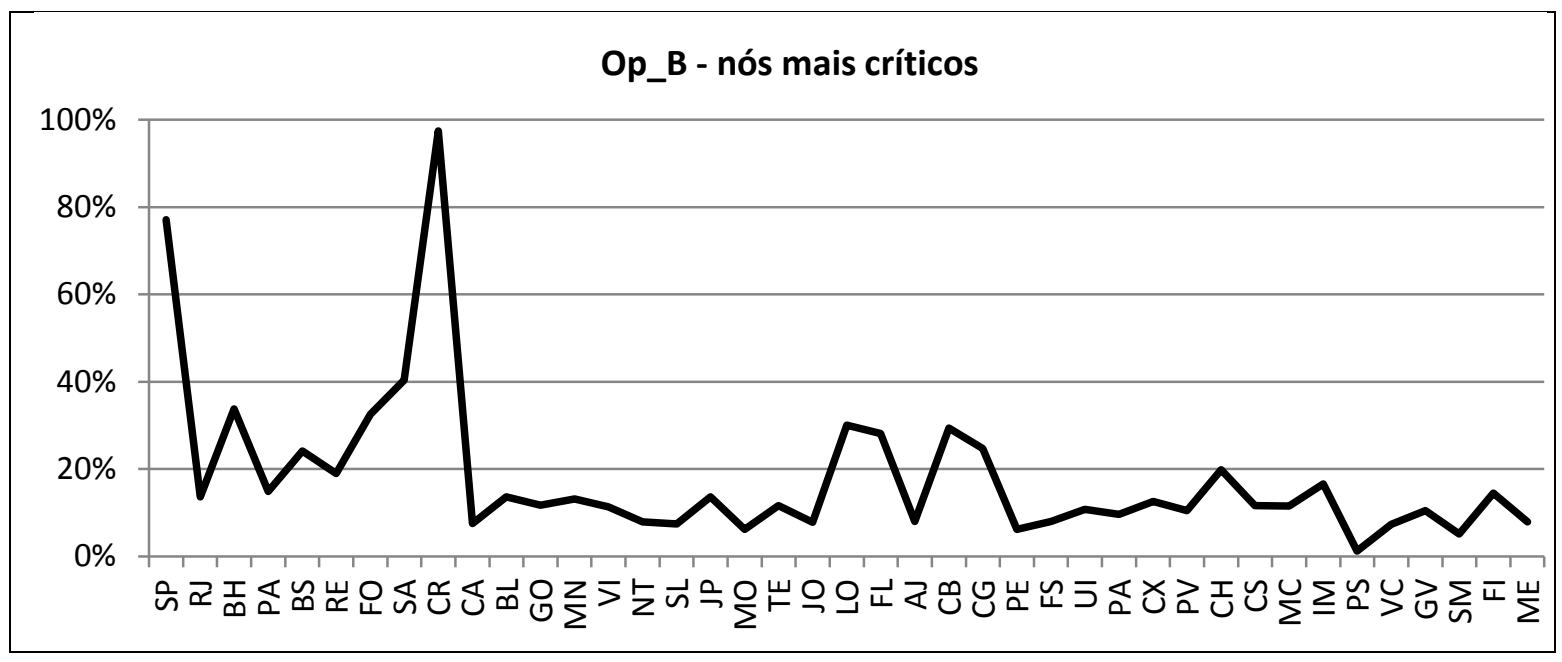

Figura 4.7 - Op_B - resultados obtidos com retirada de nós

Complementando a análise, é evidente no mapa da Figura 4.6 que o enlace SP-CR (São Paulo-SP a Curitiba-PR) é crítico para a conectividade. O gráfico da Figura 4.8 quantifica o impacto da perda desse enlace, que é superior ao da perda e quaisquer outros nós da rede Op_B, com exceção apenas dos dois nós mais críticos, SP e CR.

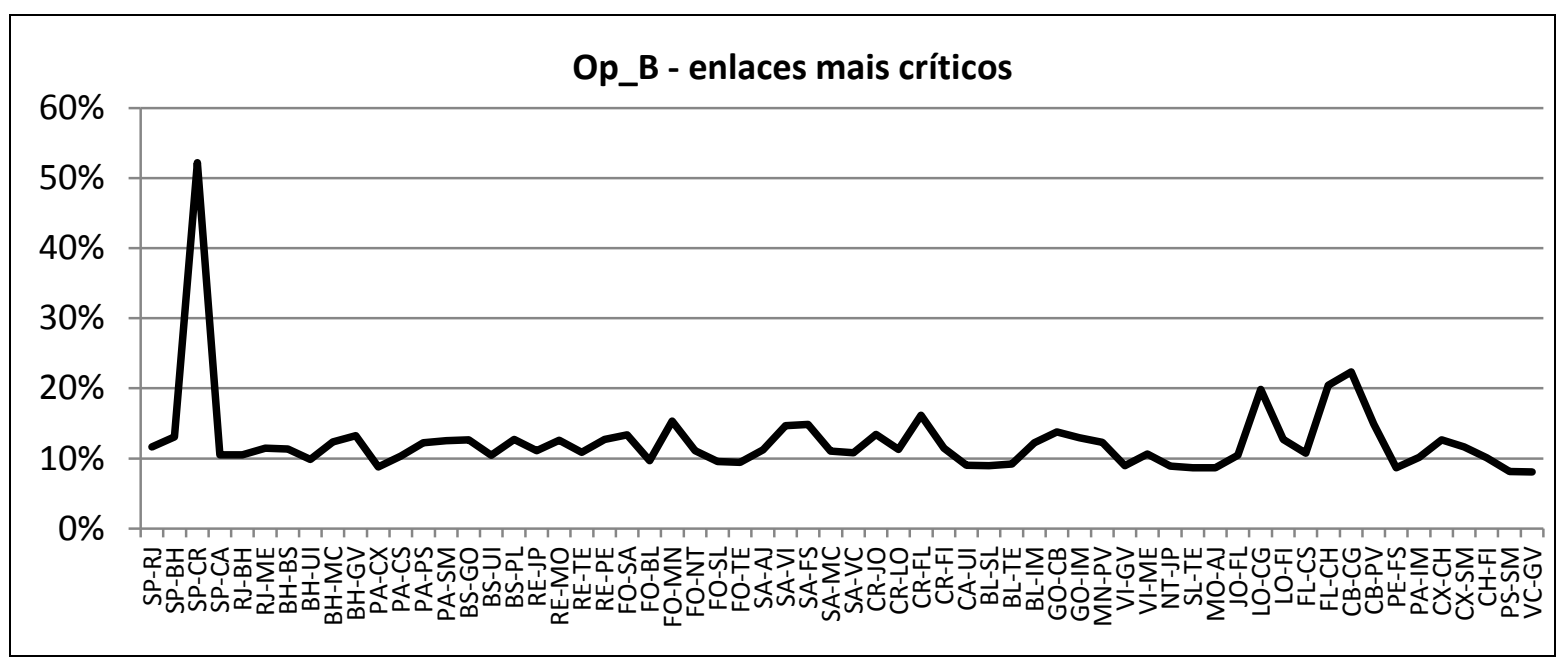

Figura 4.8 - Op_B - resultados obtidos com retirada de enlaces

Assim, fica claro que os nós SP e CR, bem como o enlace SP-CR são "pontos críticos” da rede Op_B. Nesse caso, a concentração geográfica desses pontos é um agravante para a vulnerabilidade desses "conectores globais" da rede.

Os demais nós e enlaces da rede são bastante menos vulneráveis. Além disso, não se observa para eles nenhuma concentração geográfica inquietante. 


\subsection{Experimentos Op_C}

O modelo utilizado para a rede Op_C está representado na Figura 4.9, na qual estão indicados os dois nós e o enlace mais críticos, conforme se explica mais abaixo.

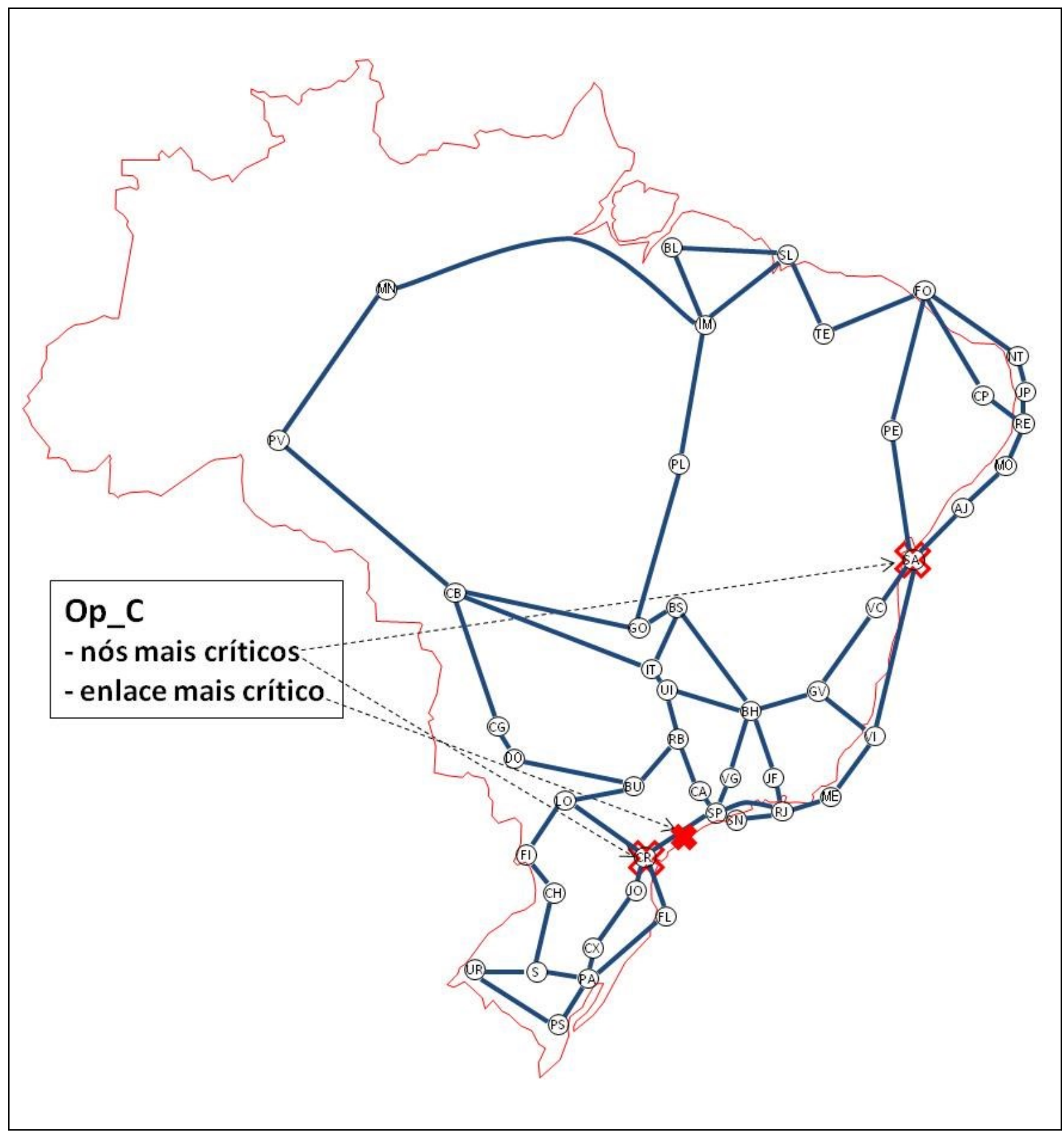

Figura 4.9 - Nós e enlaces mais críticos da rede Op_C

A métrica indica que os dois nós mais críticos são SA (Salvador-BA) e CR (Curitiba-PA). Por simples observação do mapa isso não é evidente, mas parece razoável. Além disso, coincide com a indicação de todas as métricas individuais, com a exceção de WS4 (que considera que há outros nós ligeiramente mais críticos, com relação a caminhos disjuntos). O nó SP (São Paulo-SP) vem em terceiro lugar, um pouco abaixo. 


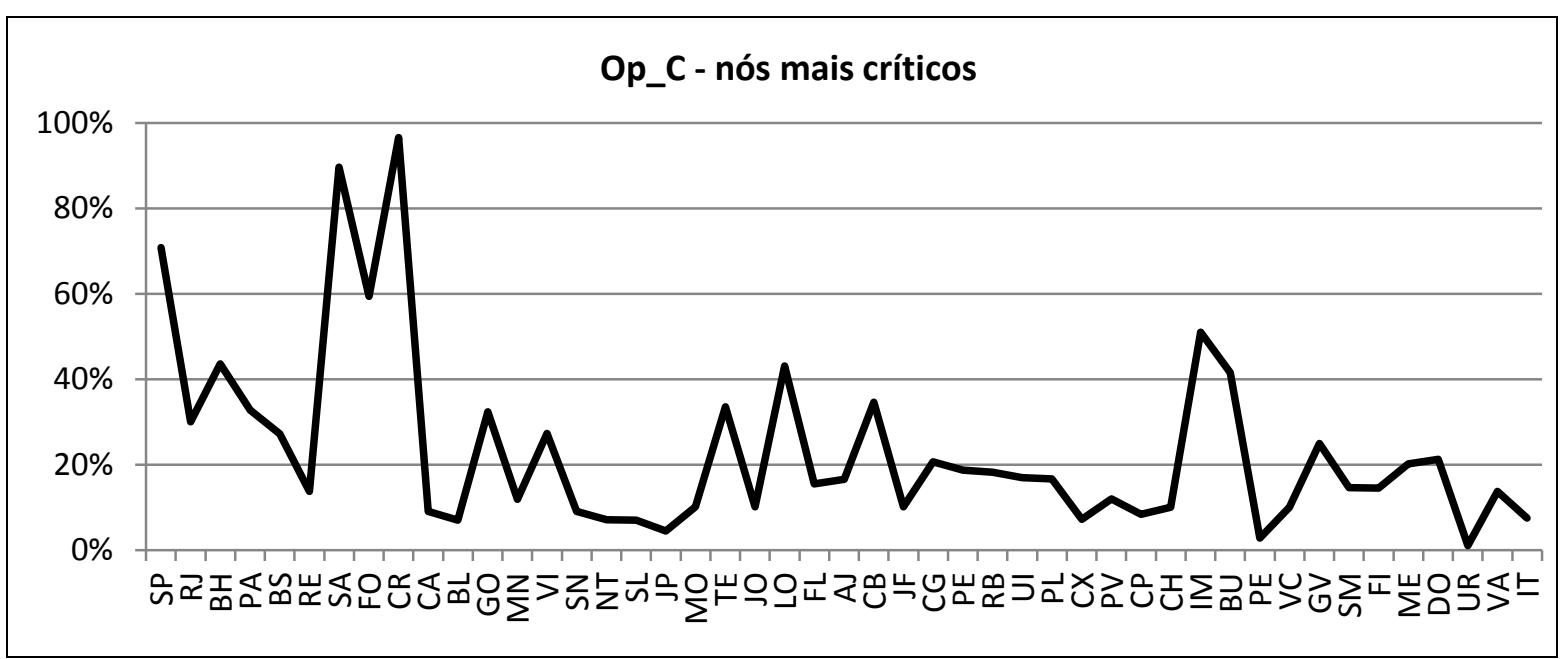

Figura 4.10 - Op_C - Resultados obtidos com retirada de nós

Já o enlace mais crítico para a conectividade da rede é mais fácil de ser identificado visualmente no mapa. O maior pico visível no gráfico da Figura 4.11 indica precisamente é o enlace SP-CR (São Paulo-SP a Curitiba-PR), seguido de vários picos bem menores..

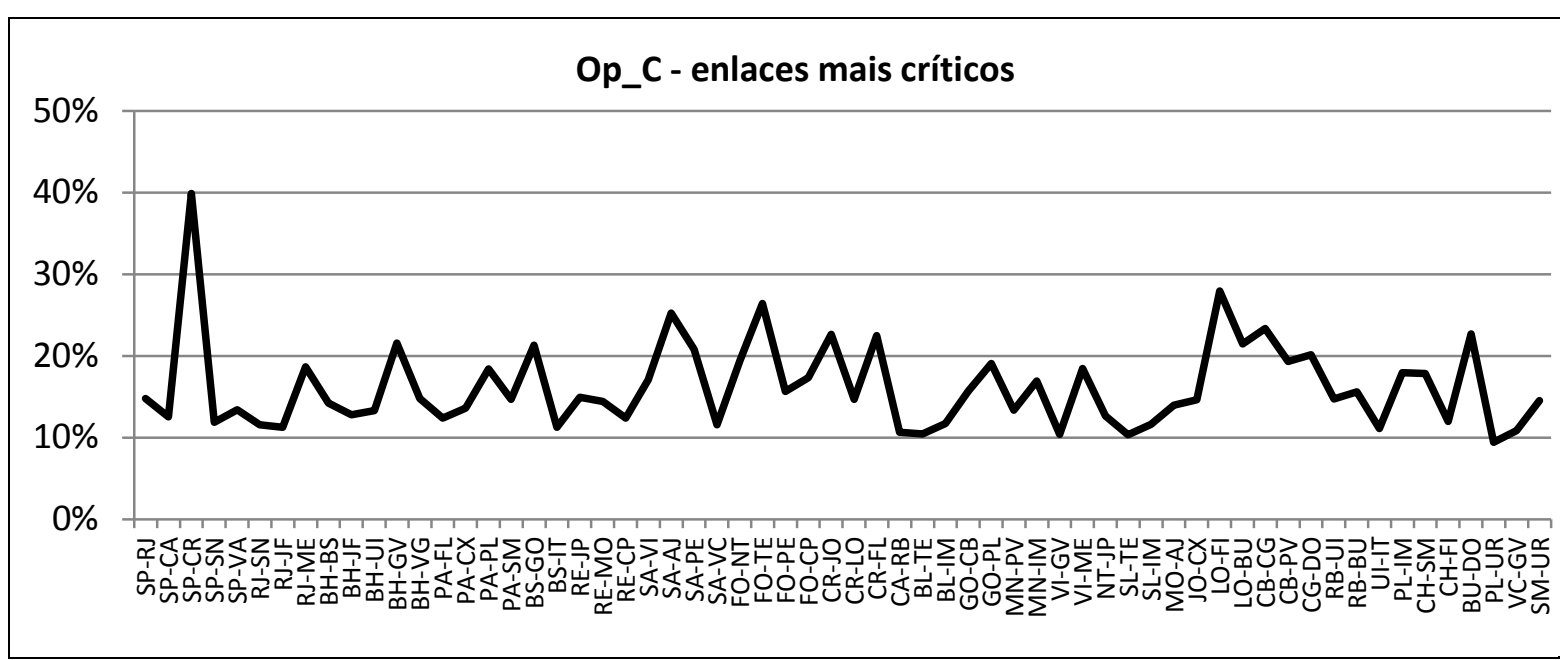

Figura 4.11 - Op_C - Resultados obtidos com retirada de enlaces

Desta forma, pode-se considerar que os "conectores globais" da rede Op_C são os nós SA e CR, juntamente com o enlace SP-CR. Quanto aos demais nós e enlaces, os picos são mais modestos e não se observam concentrações geográficas relevantes quando esses picos secundários das Figuras 4.10 e 4.11 são referidos ao mapa da Figura 4.9.

Comparada com as outras redes até aqui analisadas, a rede Op_C mostra-se mais homogênea, com diferenças menos pronunciadas entre seus nós ou enlaces mais críticos e os demais. Dito de outra forma, os picos principais e secundários observáveis nos gráficos das Figuras 4.10 e 4.11 estão mais parecidos e distribuídos de forma mais regular. 


\subsection{Experimentos Op_D}

O modelo utilizado para a rede Op_D está representado na Figura 4.12, na qual estão indicados os dois nós e os dois enlaces mais críticos, conforme discutido mais abaixo.

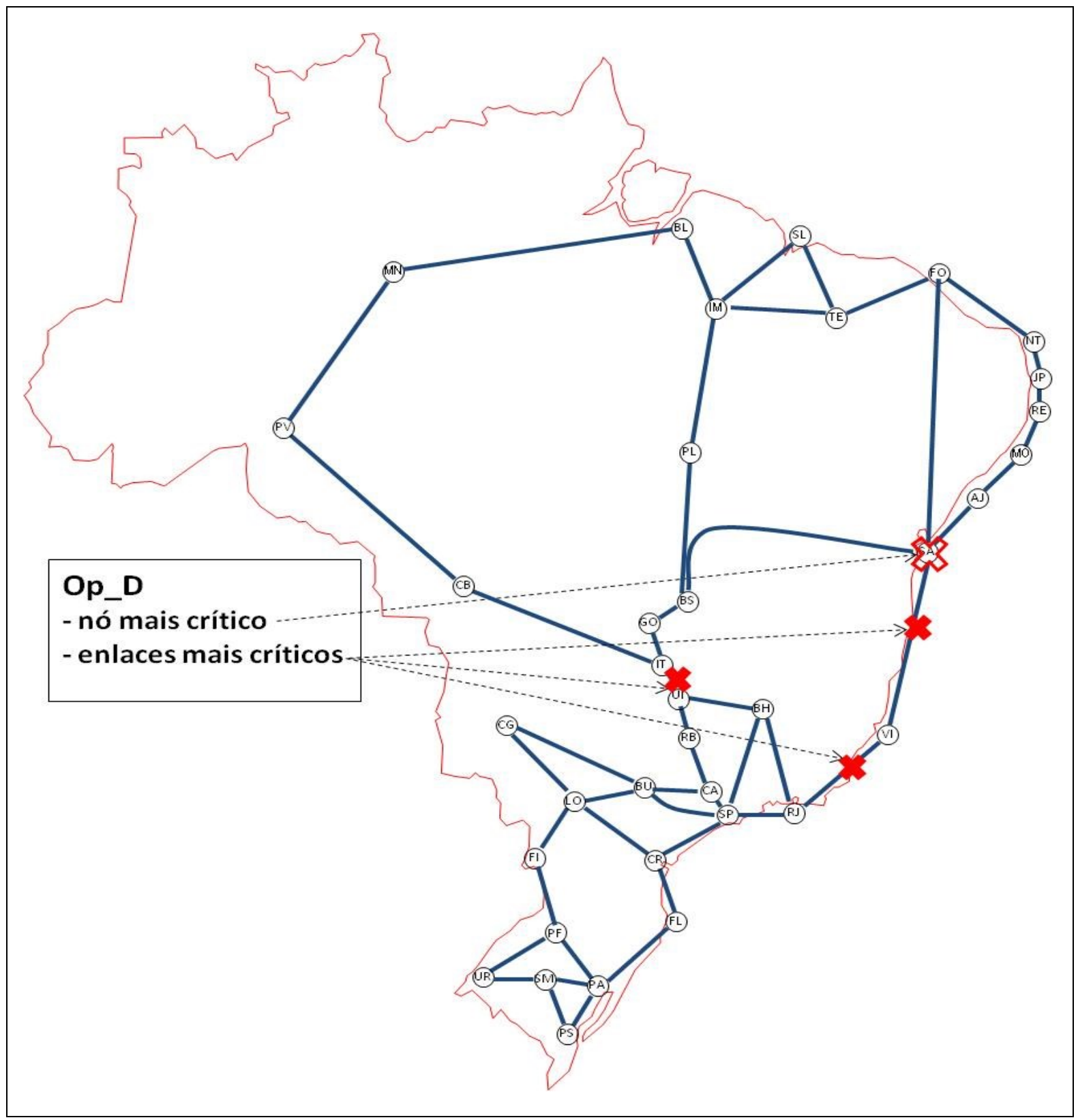

Figura 4.12 - Nós e enlaces mais críticos da rede Op_D

No caso da rede Op_D, o nó mais crítico é SA (Salvador-BA), o que é bem intuitivo ao se observar no mapa da Figura 4.12. Em segundo lugar está o nó SP (São Paulo-SP), conforme indica o segundo maior pico do gráfico da Figura 4.13. Isso não é tão evidente por simples observação visual, mas todas as métricas individuais concordam em que esse nó ocupa o segundo lugar, em termos de seu impacto na vulnerabilidade da rede. 


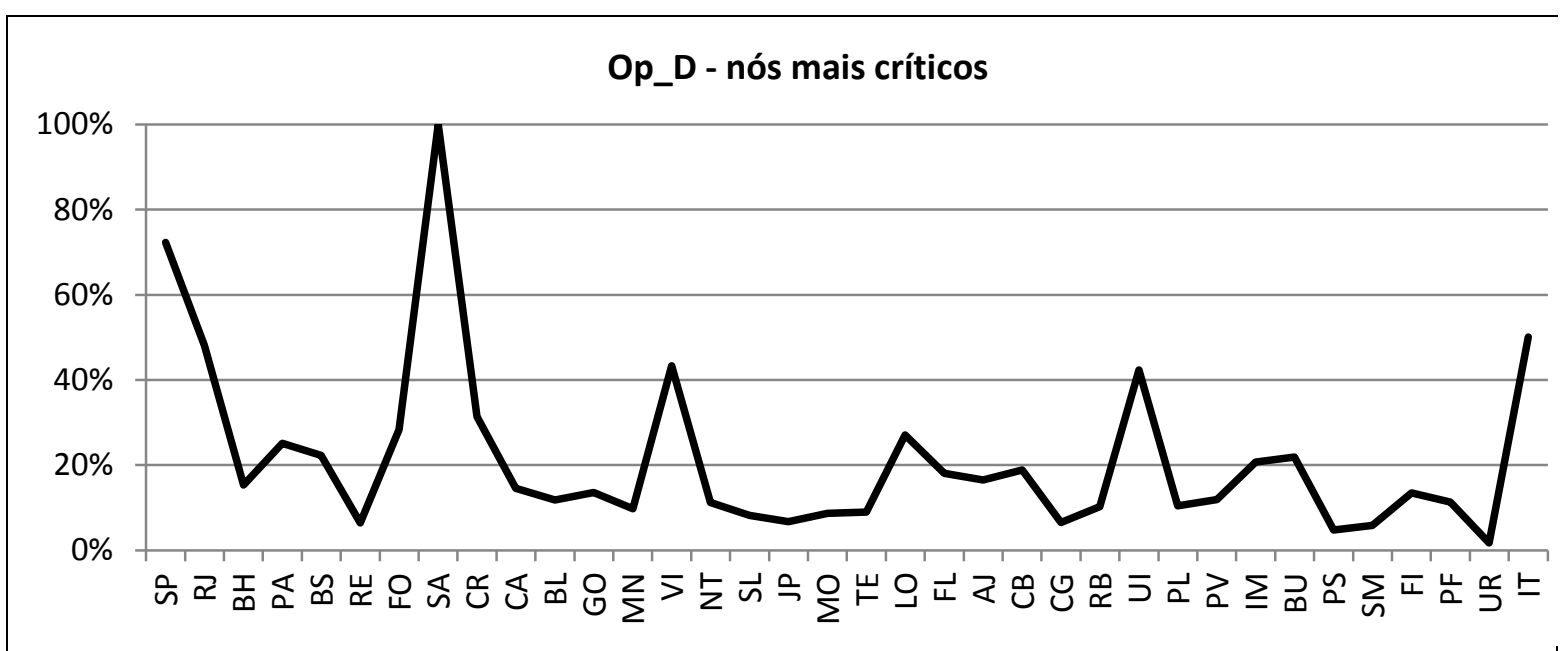

Figura 4.13 - Op_D - Resultados obtidos com retirada de nós

Com relação aos enlaces críticos, é fácil observar na Figura 4.12 que a rede Op_D possui três enlaces visivelmente críticos, que são UI-IT (Uberlândia-MG a Itumbiara-GO), SA-VI (Salvador-BA a Vitória-ES) e RJ-VI (Rio de Janeiro-RJ a Vitória-ES). O gráfico da Figura 4.14 indica que há pouca diferença entre os impactos na vulnerabilidade da rede provocados por cada um deles. Observa-se além disso que a intensidade de tais impactos não é tão expressiva, três vezes inferior à do nó mais crítico (a métrica composta $C$ utiliza a mesma escala normalizada de 0 a 100\% para os nós e enlaces de uma mesma rede).

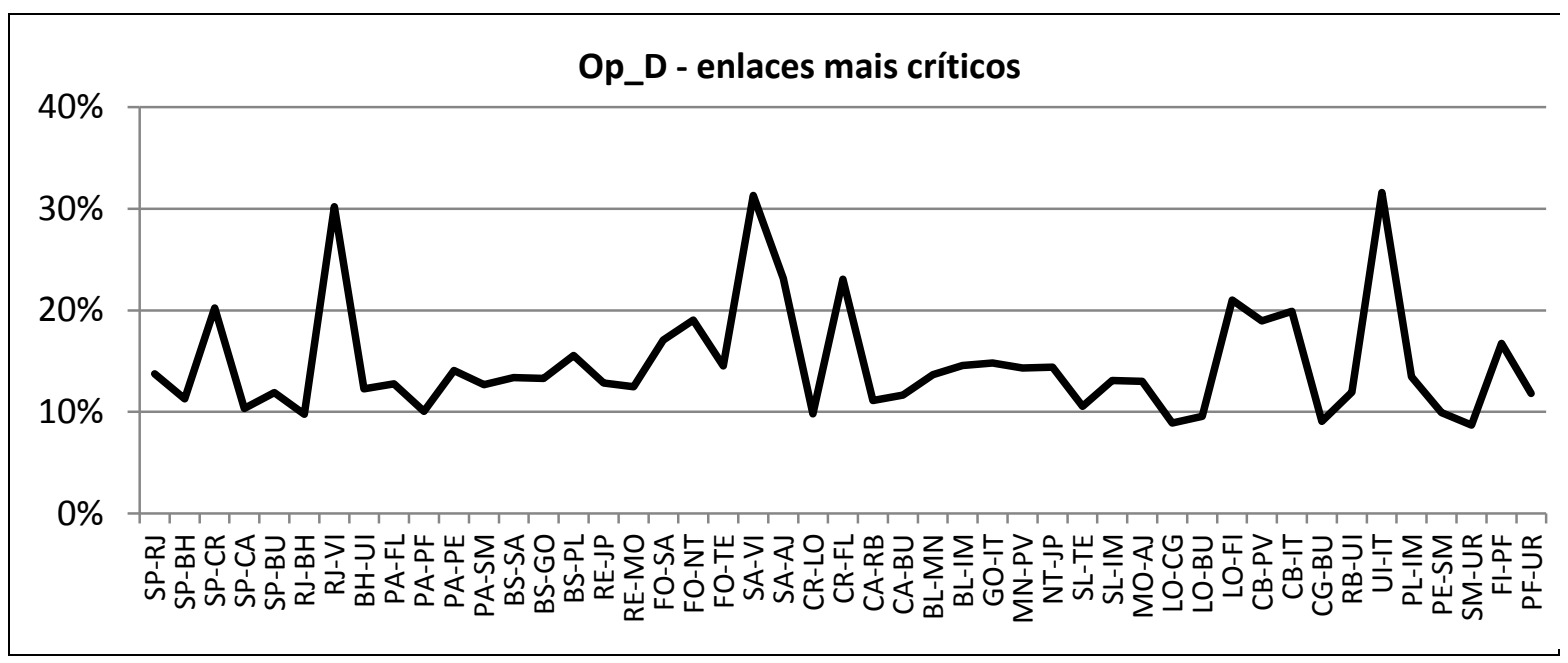

Figura 4.14 - Op_D - Resultados obtidos com retirada de enlaces

Desta forma, pode-se concluir que o único "conector global" identificado para a rede Op_D seria o nó SA. Os demais nós e enlaces têm impactos bem mais modestos e os picos secundários observáveis nos gráficos das Figuras 4.13 e 4.14 estão razoavelmente distribuídos, não se concentrando em nenhuma região geográfica em particular. 


\subsection{Experimentos Op_E}

O modelo utilizado para a rede Op_E está representado na Figura 4.15, na qual estão indicados o nó e os dois enlaces mais críticos, conforme se analisa mais abaixo.

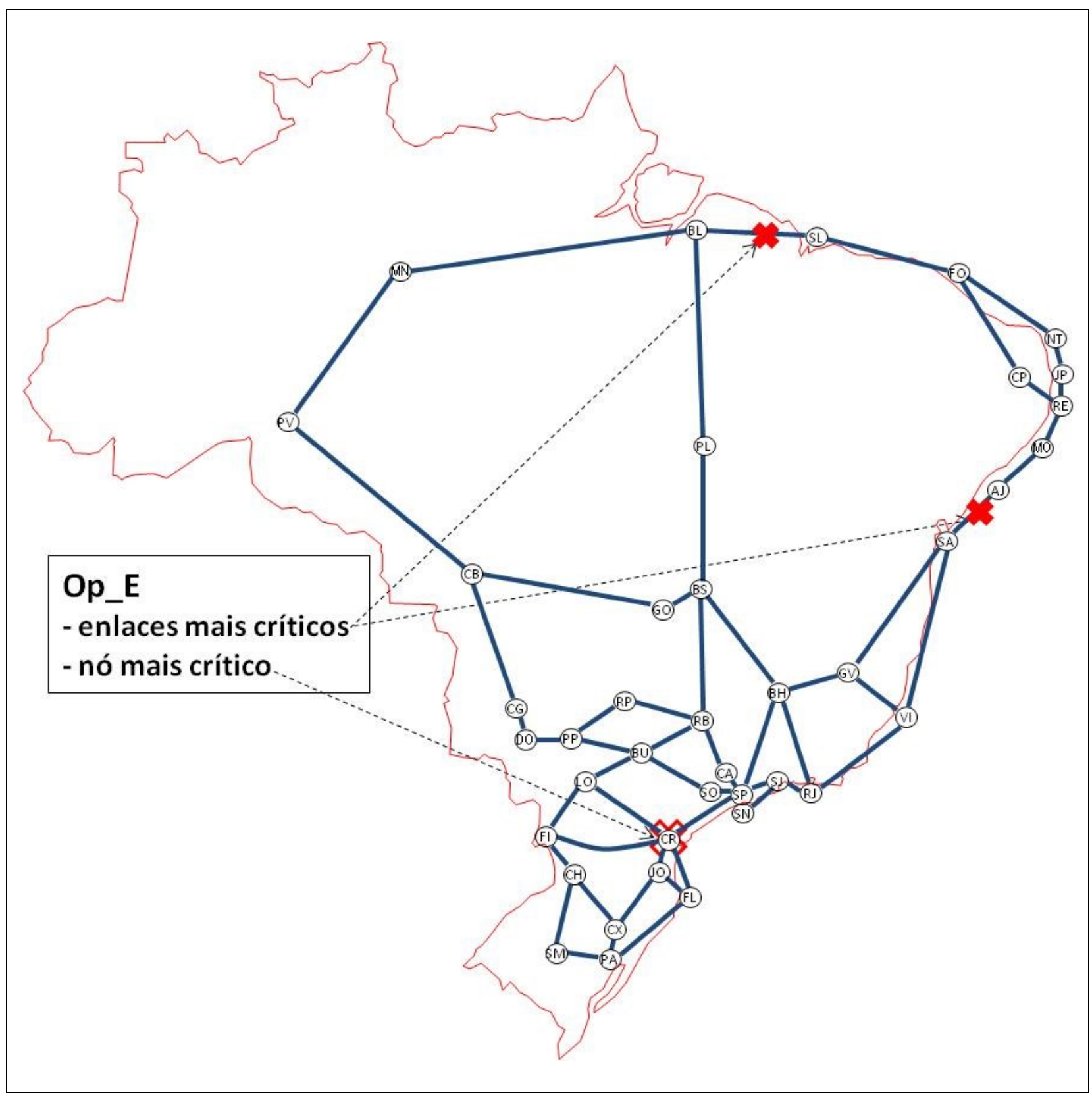

Figura 4.15 - Nós e enlaces mais críticos da rede Op_E

No caso da rede Op_E, o nó mais crítico é CR (Curitiba-PR) de acordo com a indicação da métrica composta $C$. Por simples inspeção visual do mapa da Figura 4.15 não seria fácil determinar quais são os nós mais vulneráveis dessa rede, nem tampouco que CR seria o nó mais crítico. De fato, observa-se que no gráfico da Figura 4.16 há vários picos secundários, que decrescem em intensidade de forma bem progressiva, indicando que há vários outros nós que estariam apenas ligeiramente abaixo de $\mathrm{CR}$ em termos de criticidade. 


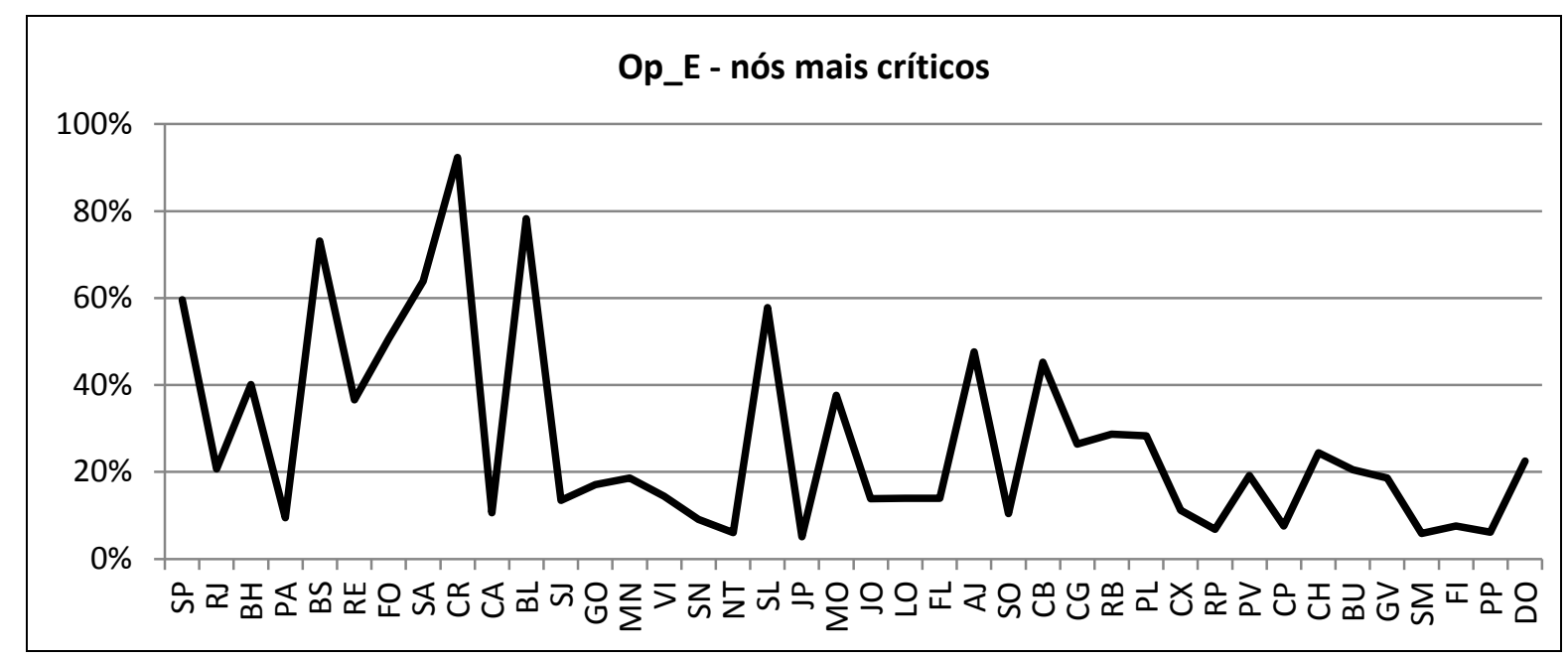

Figura 4.16 - Op_E - Resultados obtidos com retirada de nós

De maneira similar, não é fácil reconhecer visualmente o enlace mais crítico para a conectividade da rede Op_E. A métrica composta C, representada no gráfico da Figura 4.17, indica que é o enlace BL-SL (Belém-PR a São Luís-MA) seguido de perto pelo enlace SA-AJ (Salvador-BA a Aracaju-SE). Nesse gráfico aparecem igualmente vários outros picos secundários, indicando outros há enlaces apenas um pouco menos críticos que os dois acima mencionados.

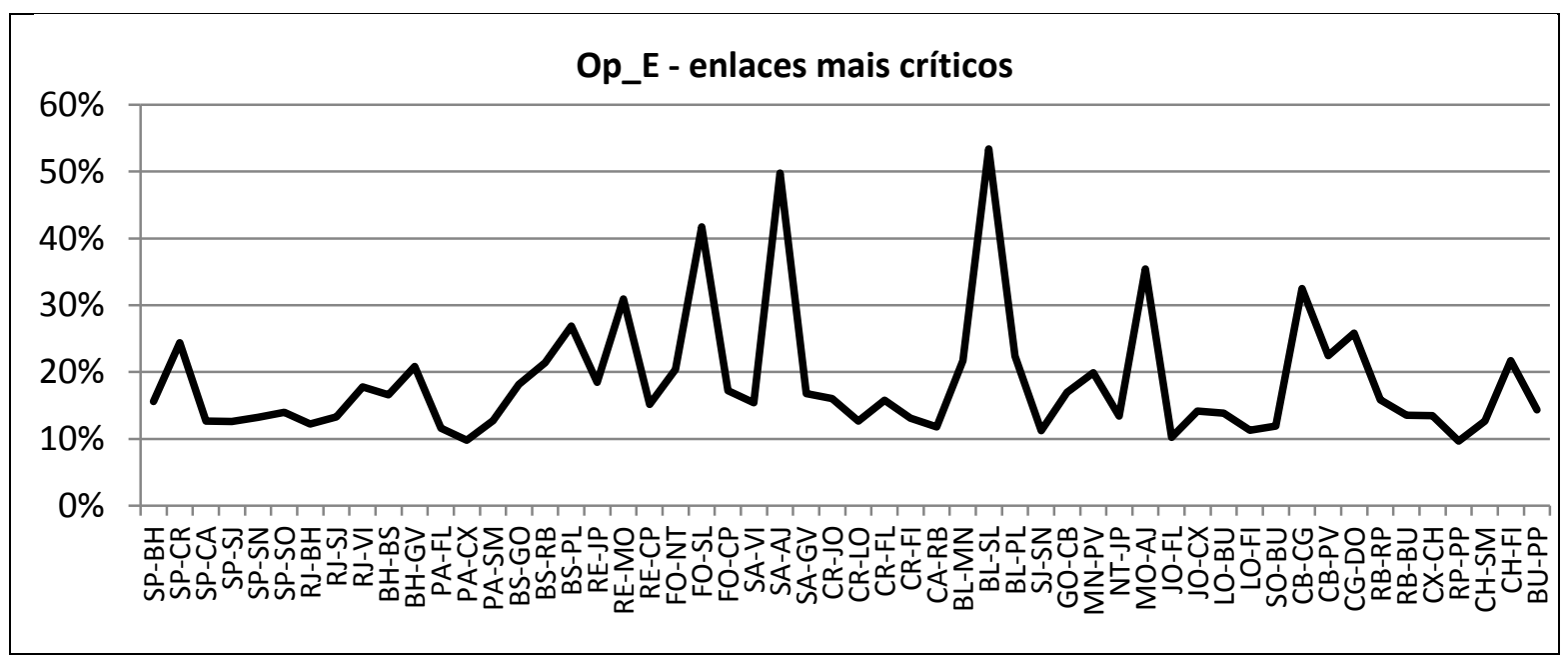

Figura 4.17 - Op_E - Resultados obtidos com retirada de enlaces

Em síntese, pode-se dizer que os conectores globais da rede Op_E seriam o nó CR e os enlaces BL-SL e SA-AJ. Os demais nós e enlaces têm impactos mais reduzidos. Por outro lado, observa-se uma relativa concentração de picos secundários na região Nordeste.

Comparada com as outras redes até aqui analisadas, a rede Op_E, mostra-se mais homogênea, com diferenças menos pronunciadas entre seus nós ou enlaces mais críticos e os demais, tal como ocorre também com a rede Op_C. 


\subsection{Experimentos Cost266}

O modelo utilizado para a rede Cost266 está representado na Figura 4.18, na qual estão indicados o nó e o enlace mais críticos, conforme se discute mais abaixo.

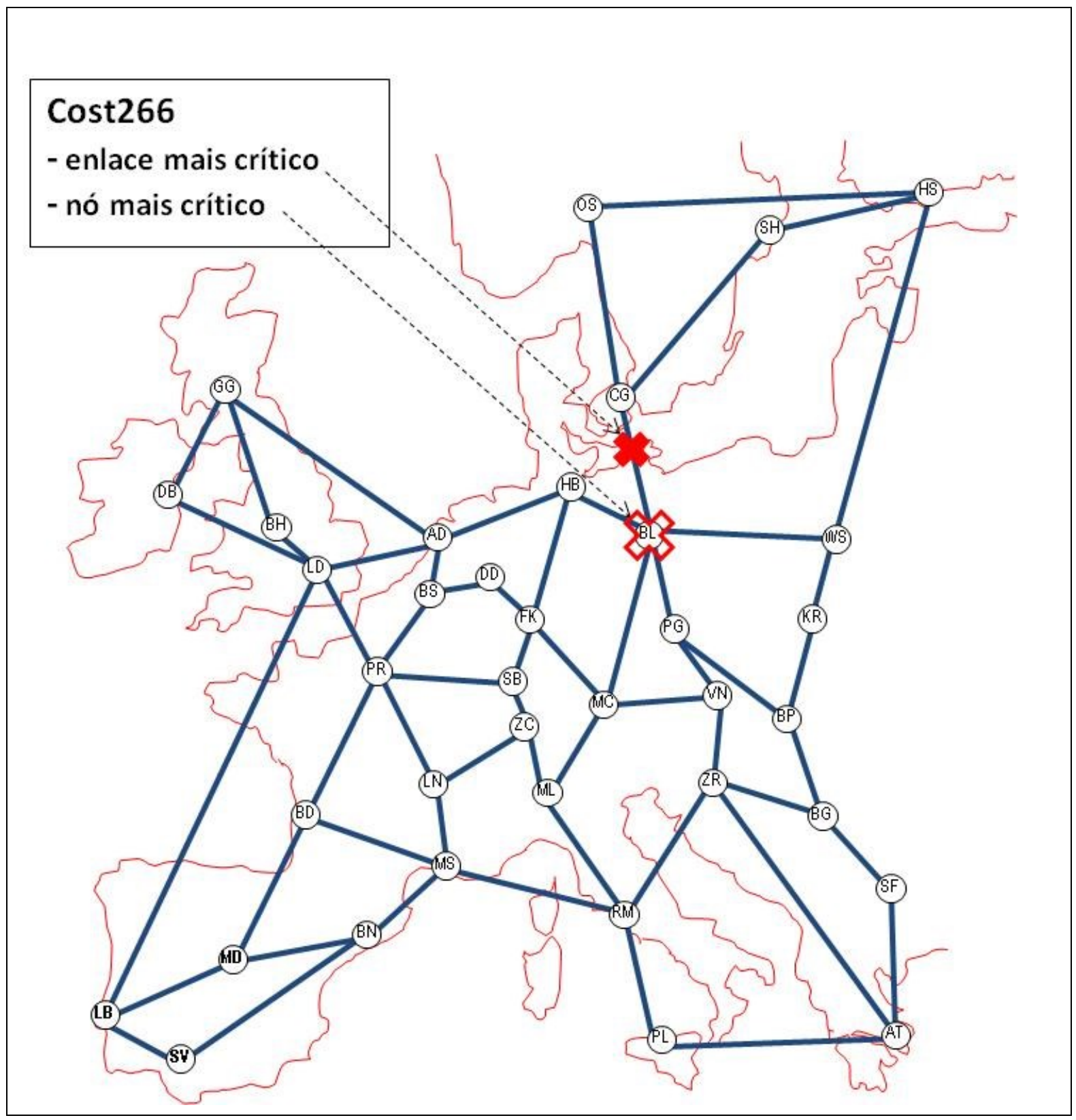

Figura 4.18 - Nós e enlaces mais críticos da rede Cost266

Por simples inspeção visual do mapa da Figura 4.18 não seria fácil determinar quais são os nós mais críticos da rede Cost266. A métrica composta $C$ representada no gráfico da Figura 4.19 deixa claro que o nó mais crítico é BL (Berlim, Alemanha), muito à frente do segundo colocado. Os diversos picos do segundo lugar em adiante se situam muito abaixo, em termos do impacto de sua remoção na conectividade da rede. 


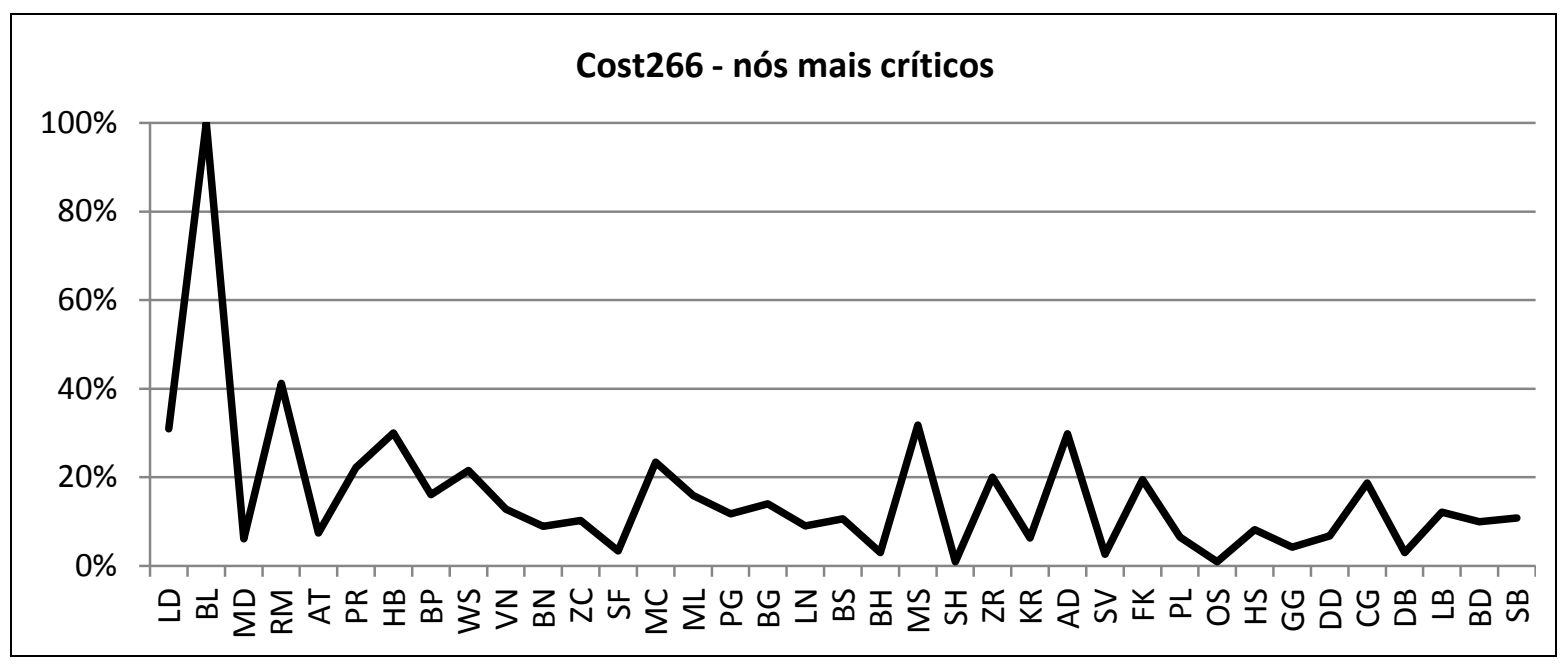

Figura 4.19 - Cost266 - Resultados obtidos com retirada de nós

De maneira similar, não é fácil reconhecer visualmente quais são os enlaces mais críticos para a conectividade da rede Cost266. A métrica $C$ mostrada no gráfico da Figura 4.20 indica que seria o enlace CG-BL (Berlim, Alemanha a Copenhagen, Dinamarca). Do segundo lugar em diante, o mesmo gráfico possui múltiplos picos, indicando que há vários outros enlaces um pouco menos críticos que o acima mencionado.

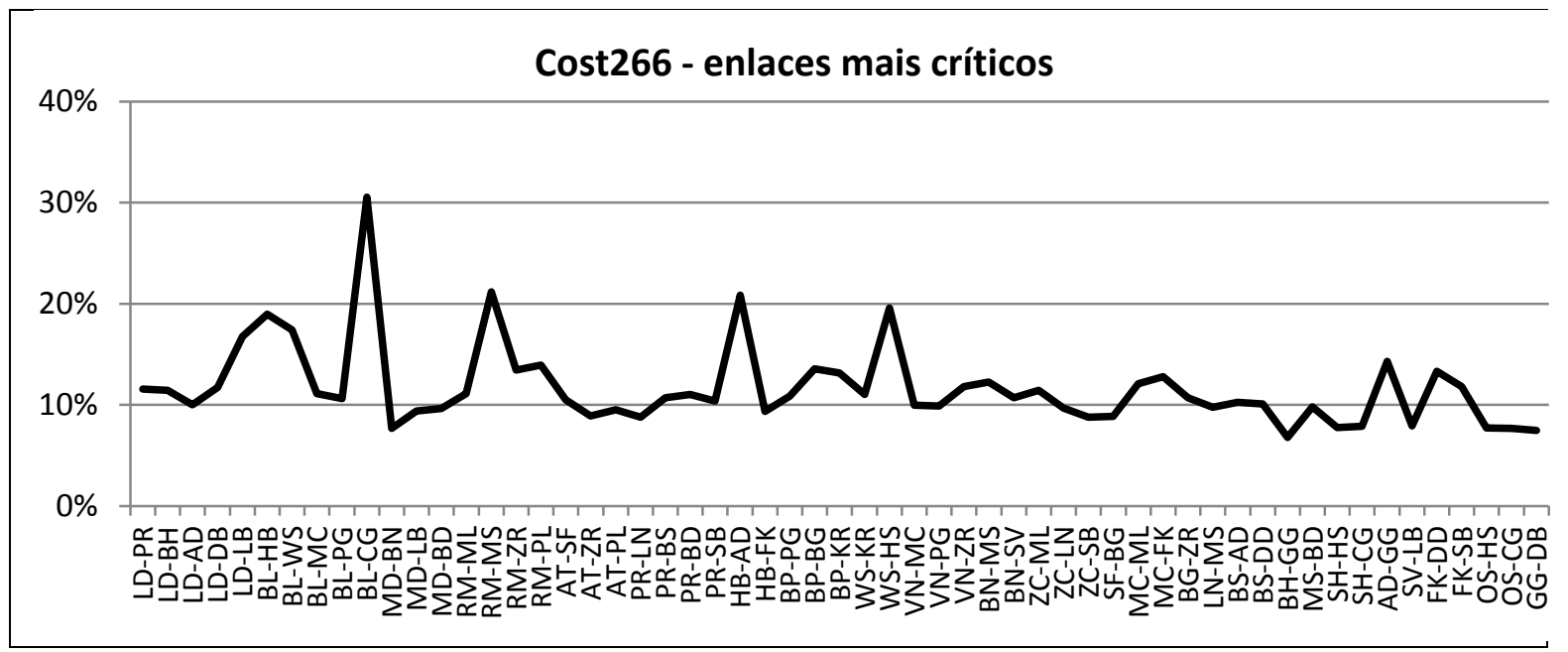

Figura 4.20 - Cost266 - Resultados obtidos com retirada de enlaces

Os resultados obtidos no experimento com a rede Cost266 indicam que o "ponto crítico" desta rede é o nó BL. A perda dos demais nós e todos os enlaces têm impactos bastante mais modestos na conectividade desta rede.

Isto coincide com o que indicam medidas similares, feitas em outros estudos, como $[43,44]$, que também realizaram experimentos com a rede Cost266 e com outras topologias de referência da SNDlib [8]. 


\subsection{Experimentos Janos US-CA}

O modelo utilizado para a rede Cost266 está representado na Figura 4.21, na qual estão indicados os dois nós e os três enlaces mais críticos, conforme se analisa mais abaixo.

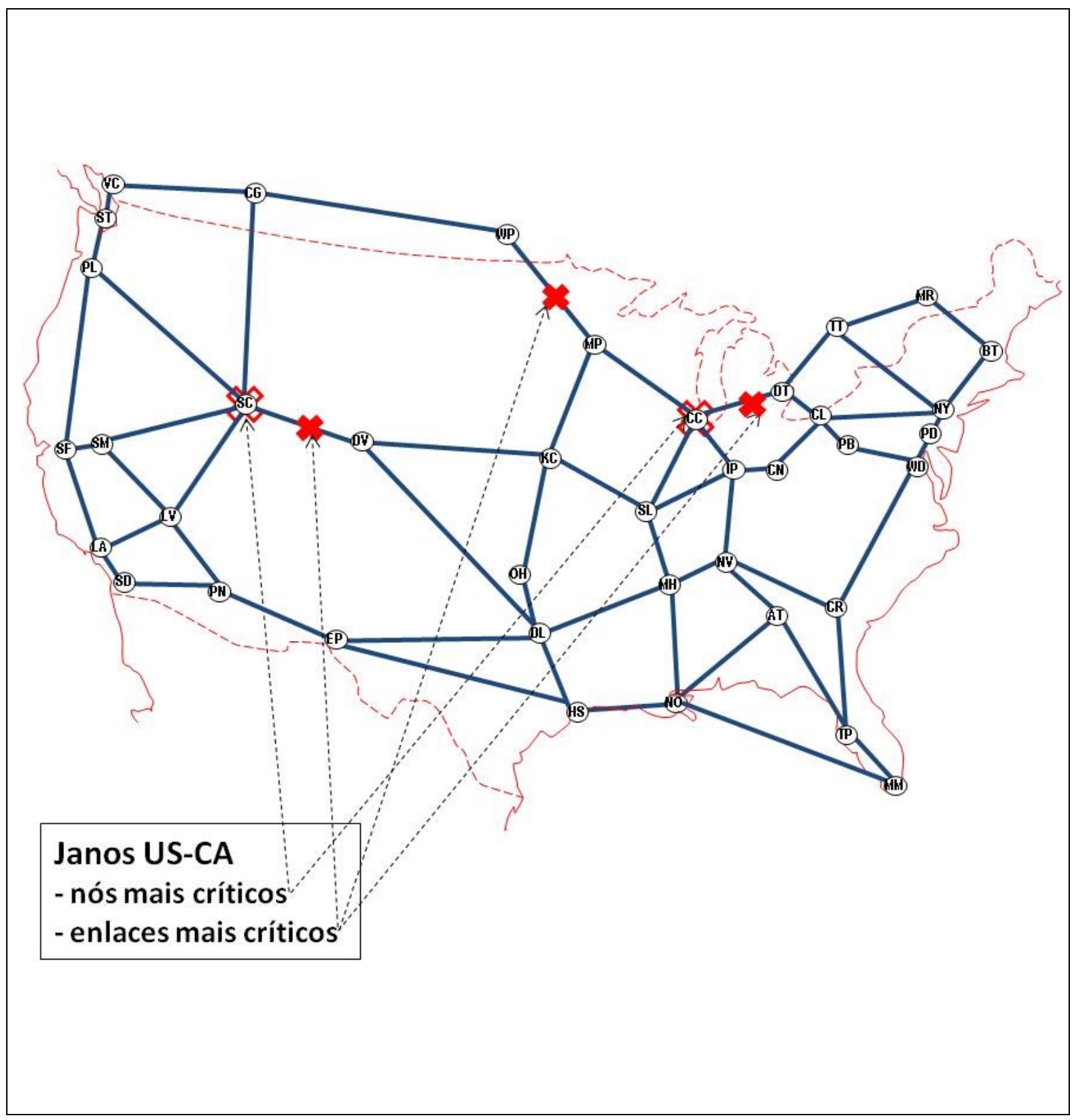

Figura 4.21 - Nós e enlaces mais críticos da rede Janos US-CA

Por simples inspeção visual do mapa da Figura 4.21, não seria fácil determinar quais são os nós mais críticos da rede Janos US-CA. Com recurso à métrica composta $C$ mostrada no gráfico da Figura 4.22, vê-se que o nó mais crítico é CC (Chicago, IL), com o nó SL (Salt Lake City, UT) ocupando o segundo lugar. A partir daí o gráfico tem vários picos, cujas intensidades diminuem progressivamente até chegar ao nó menos crítico. 


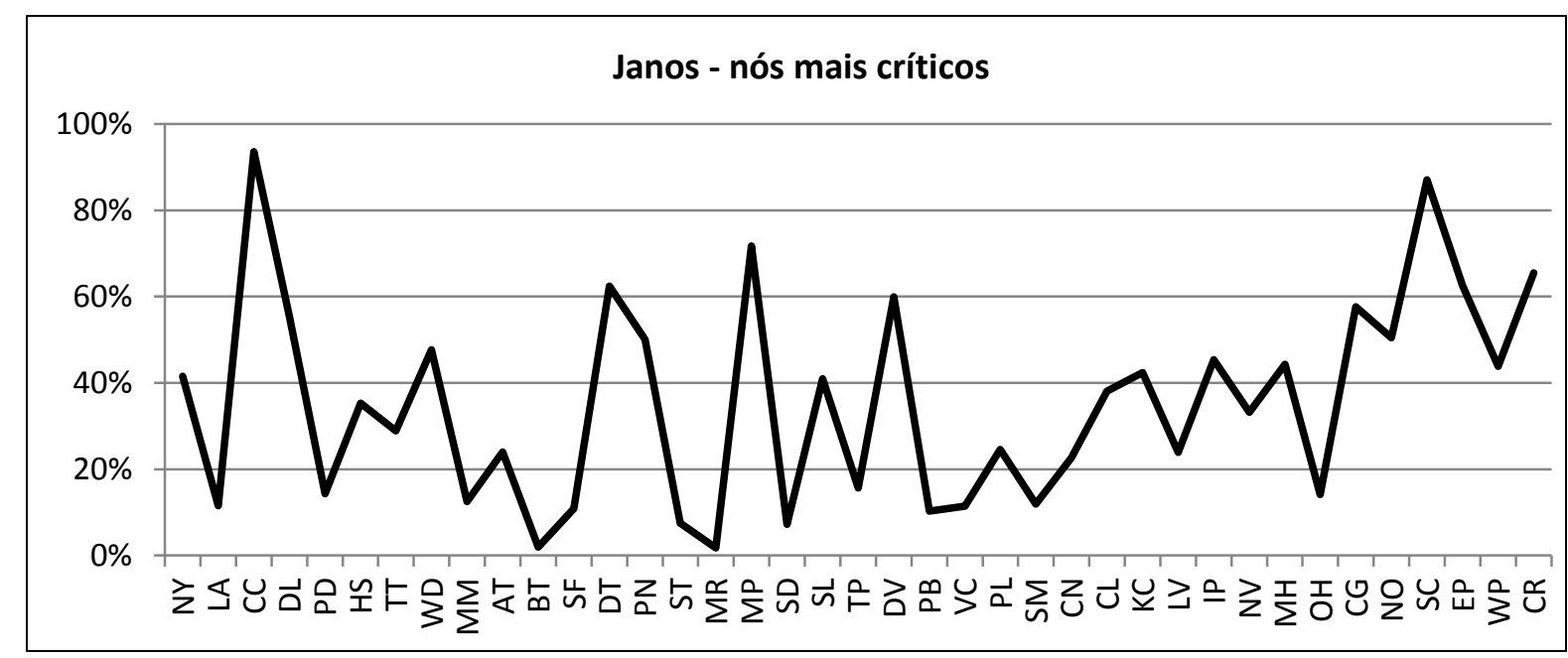

Figura 4.22 - Janos US-CA - Resultados obtidos com retirada de nós

De maneira similar, não é fácil reconhecer visualmente na Figura 4.21 quais são os enlaces mais críticos para a conectividade da rede Janos US-CA. A métrica composta $C$, representada no gráfico da Figura 4.23, indica que é o enlace MP-WP (Minneapolis, MN a Winnipeg, Canadá), seguido pelos enlaces CC-DT (Chicago, IL a Detroit, MI) e SC-DV (Salt Lake City, UT a Denver, CO). O gráfico possui ainda vários outros picos menores, indicando que há outros enlaces cuja perda teria impacto forte na conectividade.

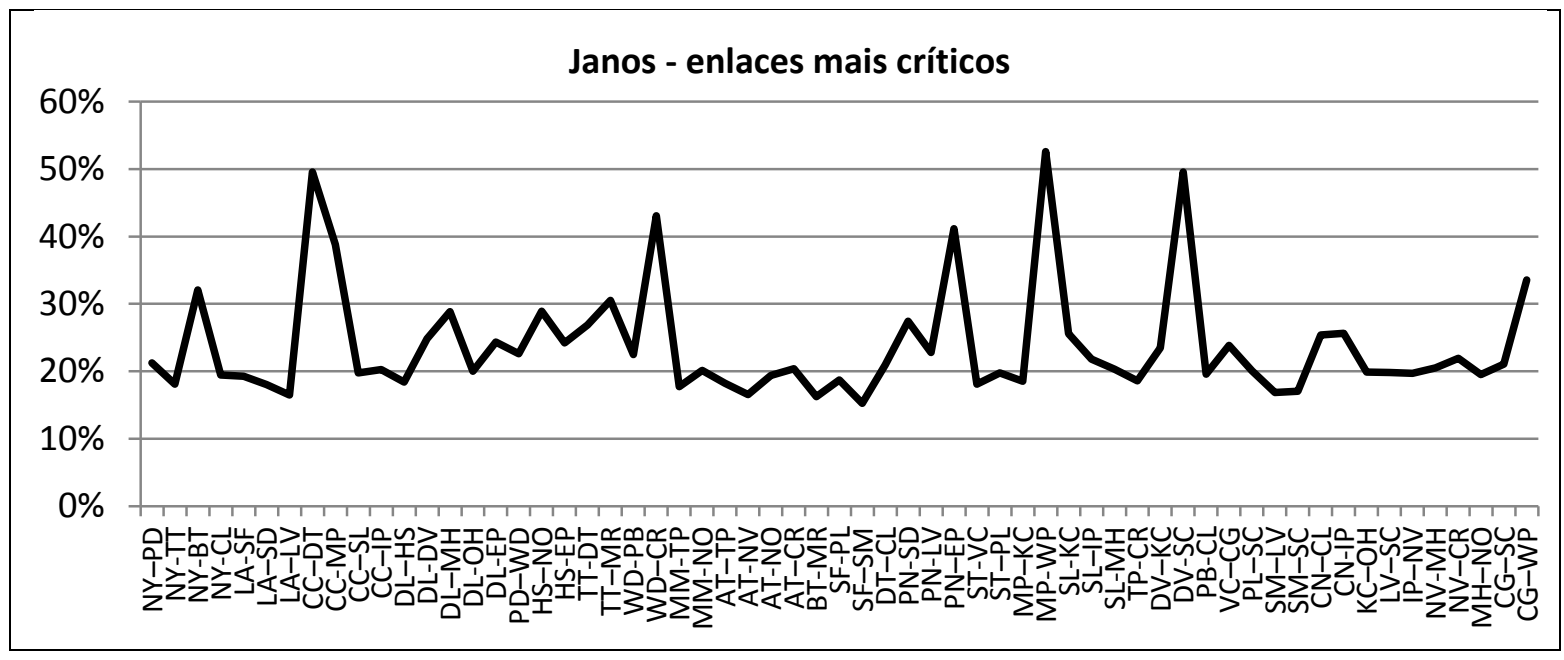

Figura 4.23 - Janos US-CA - Resultados obtidos com retirada de enlaces

Em síntese, o nó CC e o enlace MP-WP podem ser considerados "pontos críticos" da rede Janos US-CA.

Isto coincide com o que indicam medidas similares, feitas em outros estudos, como $[43,44]$, que também realizaram experimentos com a rede Cost266 e com outras topologias de referência da SNDlib [8]. 


\subsection{Comparação entre distintas redes}

As Seções 4.1 a 4.7 apresentaram resultados relativos à supressão de nós ou enlaces dentro de uma mesma rede. Isso já permite tirar algumas conclusões globais e fazer certas comparações entre essas redes.

Por exemplo, no caso das redes brasileiras nota-se que o nó SA (Salvador-BA) e o enlace SP-CR (São Paulo-SP a Curitiba-PR) aparecem como "pontos críticos" com maior frequência que os demais. Mesmo nos casos em que não ocupam o primeiro lugar, esses pontos nunca deixam de aparecer em picos com ranking considerável.

Uma observação válida para todas as redes é a de que os pontos mais críticos encontrados nos experimentos não costumam ser os mais óbvios, nem aqueles que se pode identificar intuitivamente, pele simples inspeção dos grafos. Os "conectores globais" não correspondem necessariamente às metrópoles mais importantes, nem aos nós mais interconectados. Tampouco são sempre os enlaces mais centrais (aqueles que aparecem mais vezes nos trajetos entre nós), nem os enlaces que parecem estar mais isolados. Por exemplo, o nó BH (Belo Horizonte-MG) e seus enlaces associados, que parecem vulneráveis, não figuram entre os mais críticos em nenhuma das redes brasileiras analisadas. Situação similar ocorre com o nó PR (Paris, França) na rede Cost266 e com o nó DL (Dallas, TX), no caso da rede Janos US-CA.

Além de comparações qualitativas, como as acima apresentadas, a partir dos dados dos experimentos pode-se além disso fazer uma comparação quantitativa e direta das vulnerabilidades das distintas redes entre si. Para isso será tomada como base a métrica composta $C$, obtida a partir da variação relativa das métricas individuais ao se retirar o nó ou o enlace mais crítico de cada uma das redes.. O maior valor observado será interpretado como sendo a rede mais vulnerável a falhas ou ataques localizados.

O ponto de partida são as métricas individuais $A S P, N C, W S 4$ e $1 / A C$. A tabela 4.9 mostra as medidas individuais obtidas para cada rede completa.

Tabela 4.9 - Medidas individuais para as redes completas

\begin{tabular}{|c|ccccccc|}
\hline $\begin{array}{c}\text { Rede / } \\
\text { métrica }\end{array}$ & Op_A & Op_B & Op_C & Op_D & Op_E & Cost 266 & $\begin{array}{c}\text { Janos } \\
\text { US-CA }\end{array}$ \\
\hline$A S P$ & 2,03 & 2,12 & 2,63 & 2,49 & 2,30 & 1,87 & 2,10 \\
$N C$ & 1,96 & 1,81 & 2,33 & 2,59 & 2,13 & 1,44 & 1,57 \\
$W S 4$ & 8,08 & 7,56 & 10,75 & 8,79 & 9,30 & 7,60 & 7,56 \\
$1 / A C$ & 9,86 & 12,20 & 15,98 & 17,41 & 11,61 & 6,31 & 9,03 \\
\hline
\end{tabular}


As tabelas 4.10 e 4.11 mostram as medidas obtidas para as mesmas redes após a supressão do nó e do enlace mais crítico de cada rede, respectivamente.

Tabela 4.10 - Medidas individuais para as redes com a remoção do nó mais crítico

\begin{tabular}{|c|ccccccc|}
\hline $\begin{array}{c}\text { Rede } / \\
\text { métrica }\end{array}$ & Op_A & Op_B & Op_C & Op_D & Op_E & Cost 266 & $\begin{array}{c}\text { Janos } \\
\text { US-CA }\end{array}$ \\
\hline$A S P$ & 2,61 & 2,73 & 3,16 & 3,46 & 2,72 & 2,34 & 2,28 \\
$N C$ & 3,38 & 3,32 & 3,57 & 4,74 & 3,33 & 2,35 & 1,88 \\
$W S 4$ & 8,83 & 8,35 & 11,40 & 9,76 & 10,28 & 8,36 & 7,89 \\
$1 / A C$ & 33,17 & 39,69 & 38,73 & 44,49 & 27,34 & 20,04 & 13,70 \\
\hline
\end{tabular}

Tabela 4.11 - Medidas individuais para as redes com a remoção do enlace mais crítico

\begin{tabular}{|c|ccccccc|}
\hline $\begin{array}{c}\text { Rede / } \\
\text { métrica }\end{array}$ & Op_A & Op_B & Op_C & Op_D & Op_E & Cost 266 & $\begin{array}{c}\text { Janos } \\
\text { US-CA }\end{array}$ \\
\hline$A S P$ & 2,17 & 2,47 & 2,85 & 2,72 & 2,42 & 1,96 & 2,17 \\
$N C$ & 2,19 & 1,91 & 2,48 & 2,38 & 2,28 & 1,62 & 1,65 \\
$W S 4$ & 8,35 & 7,80 & 11,07 & 9,07 & 9,77 & 8,08 & 7,78 \\
$1 / A C$ & 17,25 & 33,98 & 27,71 & 31,73 & 25,52 & 9,62 & 12,11 \\
\hline
\end{tabular}

As Tabelas 4.12 e 4.13 mostram as variações relativas das medidas para as mesmas redes após a supressão do nó e do enlace mais crítico, respectivamente. Cada variação absoluta é dividida por seu valor de referência (rede completa). Como requerido para as funções de utilidade individuais, todas essas variações são expressas em uma mesma escala normalizada, com valores entre 0 e $100 \%$.

Tabela 4.12 - Medidas individuais para as redes com a remoção do nó mais crítico

\begin{tabular}{|c|ccccccc|}
\hline $\begin{array}{c}\text { Rede } / \\
\text { métrica }\end{array}$ & Op_A & Op_B & Op_C & Op_D & Op_E & Cost 266 & $\begin{array}{c}\text { Janos } \\
\text { US-CA }\end{array}$ \\
\hline$\Delta A S P$ & $15 \%$ & $15 \%$ & $12 \%$ & $19 \%$ & $11 \%$ & $14 \%$ & $7 \%$ \\
$\Delta N C$ & $33 \%$ & $37 \%$ & $25 \%$ & $37 \%$ & $26 \%$ & $29 \%$ & $11 \%$ \\
$\Delta W S 4$ & $7 \%$ & $8 \%$ & $6 \%$ & $8 \%$ & $8 \%$ & $7 \%$ & $5 \%$ \\
$\Delta(1 / A C)$ & $100 \%$ & $95 \%$ & $62 \%$ & $67 \%$ & $59 \%$ & $92 \%$ & $24 \%$ \\
\hline
\end{tabular}

Tabela 4.13 - Medidas individuais para as redes com a remoção do enlace mais crítico

\begin{tabular}{|c|ccccccc|}
\hline $\begin{array}{c}\text { Rede } / \\
\text { métrica }\end{array}$ & Op_A & Op_B & Op_C & Op_D & Op_E & Cost 266 & $\begin{array}{c}\text { Janos } \\
\text { US-CA }\end{array}$ \\
\hline$\Delta A S P$ & $6 \%$ & $10 \%$ & $7 \%$ & $7 \%$ & $5 \%$ & $5 \%$ & $5 \%$ \\
$\Delta N C$ & $8 \%$ & $6 \%$ & $6 \%$ & $0 \%$ & $6 \%$ & $9 \%$ & $5 \%$ \\
$\Delta W S 4$ & $5 \%$ & $5 \%$ & $5 \%$ & $5 \%$ & $5 \%$ & $6 \%$ & $5 \%$ \\
$\Delta(1 / A C)$ & $34 \%$ & $76 \%$ & $33 \%$ & $37 \%$ & $52 \%$ & $25 \%$ & $17 \%$ \\
\hline
\end{tabular}


A Tabela 4.14 mostra os valores da métrica composta $C$ em cada uma das redes, para os casos de perda do nó mais crítico (C-nó) e do enlace mais crítico (C-enlace). Os valores são obtidos a partir dos dados das Tabelas 4.12 e 4.13 , respectivamente, utilizando a equação 4.1 .

Tabela 4.14 - Medidas individuais para as redes com a remoção do nó mais crítico

\begin{tabular}{|c|ccccccc|}
\hline $\begin{array}{c}\text { Rede } / \\
\text { métrica }\end{array}$ & Op_A & Op_B & Op_C & Op_D & Op_E & Cost 266 & $\begin{array}{c}\text { Janos } \\
\text { US-CA }\end{array}$ \\
\hline C-nó & $49 \%$ & $48 \%$ & $32 \%$ & $40 \%$ & $31 \%$ & $45 \%$ & $14 \%$ \\
C-enlace & $16 \%$ & $31 \%$ & $16 \%$ & $15 \%$ & $22 \%$ & $13 \%$ & $9 \%$ \\
\hline
\end{tabular}

O valores de $C$ obtidos para cada rede são mostrados no gráfico da Figura 4.24, que permite uma boa visualização de suas vulnerabilidades relativas.

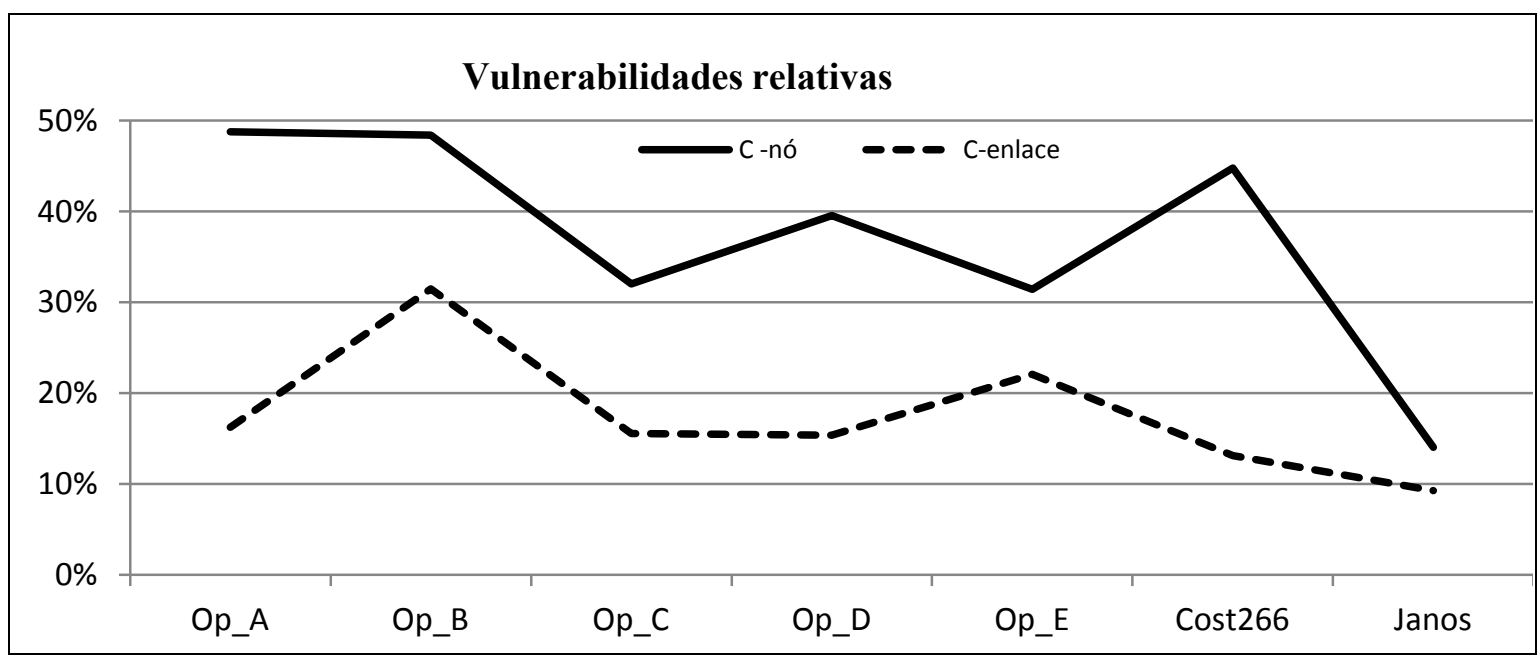

Figura 4.24 - Impactos da perda do nó ou do enlace mais crítico

O gráfico da Fig. 4.24 mostra que, do ponto de vista do impacto do seu nó mais crítico, a rede Op_A seria a mais vulnerável, praticamente empatada com a rede Op_B, seguida pelas redes Cost266, Op_C, Op_D, Op_E e Janos US-CA, nesta ordem. Do ponto de vista do impacto da retirada do enlace mais crítico, a rede Op_B seria a mais vulnerável, seguida pela rede Op_E, pelas redes Op_A, Op_C e Op_D (quase empatadas), Cost266 e Janos US-CA, nesta ordem.

Conforme já foi observado, para uma mesma rede o impacto da retirada do nó mais crítico é sempre maior que a de seu enlace mais crítico. No entanto, é importante identificar os enlaces críticos de forma independente dos nós críticos, posto que os tipos de problemas que ocorrem com enlaces são distintos dos que ocorrem com os nós. Os enlaces 
podem ser mais susceptíveis a falhas correlacionadas a geografia que os nós, devido à sua extensão física e maior exposição ao ambiente. Por exemplo, as fibras óticas podem sofrer danos por escavações, vandalismo ou pela incidência de raios em cabos OPGW.

As redes mais vulneráveis não são sempre as mais óbvias, da mesma forma do que ocorre com a retirada de nós ou enlaces dentro de uma mesma rede. No caso da comparação entre distintas redes é ainda mais difícil reconhecer aquelas que são mais vulneráveis por simples inspeção de seus grafos. Não corresponde necessariamente às redes menos densas, as menos simétricas ou as que possuem mais aglomerações (clusters). Por exemplo, por simples inspeção, a rede Cost266 parece muito menos vulnerável do que indicam suas medidas. O contrário ocorre com a rede Op_A, que visualmente parece muito mais vulnerável à perda de um enlace crítico do que indica sua medida correspondente.

A Figura 4.25 permite visualizar em conjunto todos os conectores globais de todas as redes ensaiadas, tal como foram identificados nas Seções 4.2 a 4.8.

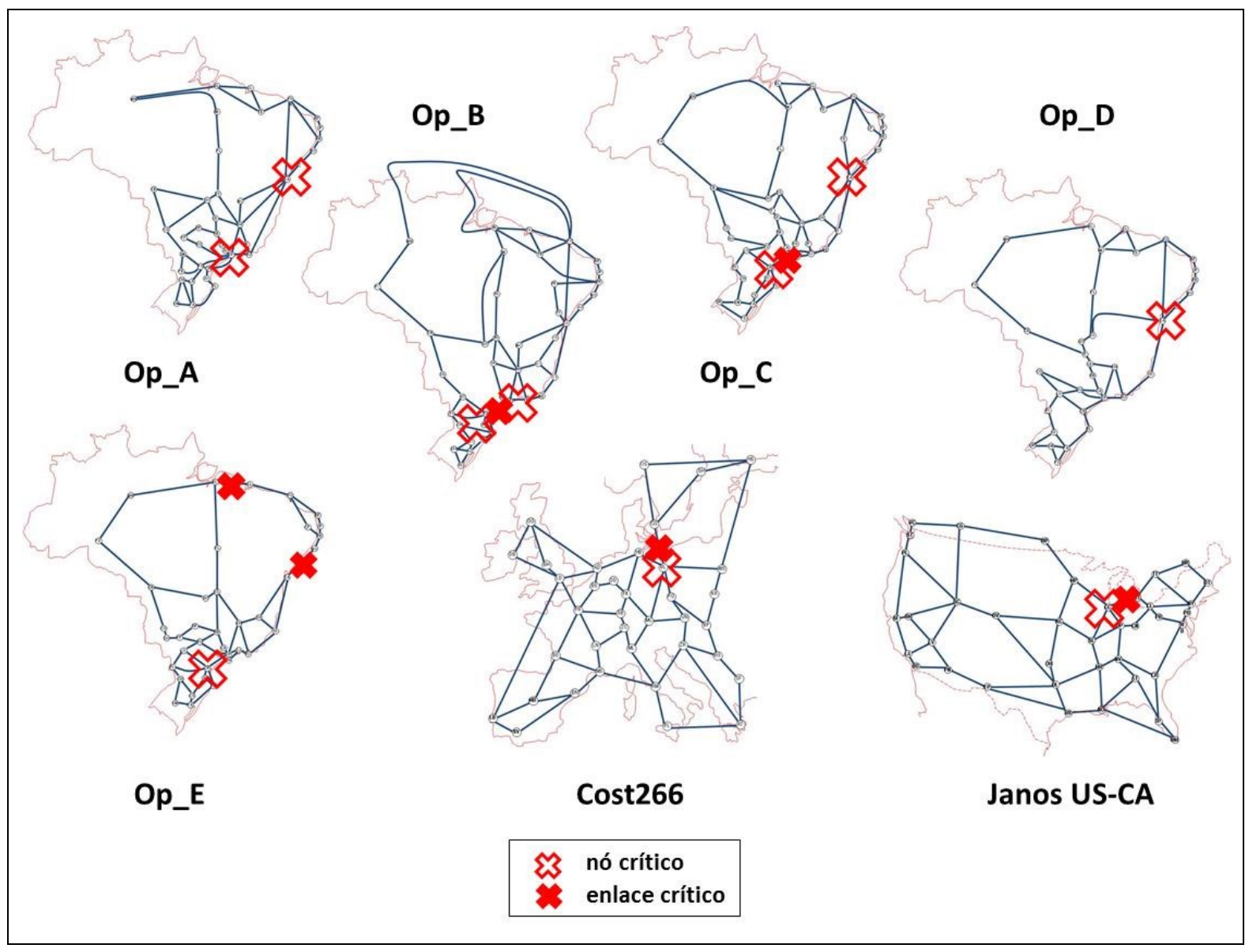

Figura 4.25 - Comparação visual entre todas as redes

A comparação visual entre todas as redes reforça as observações da presente seção. 


\section{CONCLUSÕES}

Este estudo gera informações quantitativas sobre a vulnerabilidade das redes de telecomunicações brasileiras que têm cobertura nacional, vistos como backbones operados de forma independente. Permite ver onde estão seus nós ou enlaces mais críticos, seus "conectores globais", cuja perda causaria a maior disrupção na rede como um todo. Foram analisadas mais de 5.000 medidas (tabuladas no Apêndice B), resultantes de experimentos realizados em cinco modelos de redes brasileiras e duas redes internacionais tomadas como referências. Os resultados obtidos dão suporte à hipótese de que a vulnerabilidade das redes de telecomunicações a falhas ou a ataques localizados pode ser quantificada e comparada a partir de uma composição adequada de métricas de conectividade.

Observa-se que os pontos mais críticos encontrados nas redes não são mais óbvios ou aqueles observáveis à primeira vista. Por exemplo, os pontos mais críticos não correspondem necessariamente às metrópoles mais importantes, aos enlaces mais centrais da rede, nem a seus nós mais conectados. Da mesma forma, tampouco as redes com maior vulnerabilidade à perda de seu nó ou enlace mais crítico costumam ser as mais óbvias. Não são necessariamente mais vulneráveis as redes menos densas, as que parecem menos simétricas, nem as que possuem mais aglomerações (clusters) e enlaces mais isolados.

Trabalhos futuros poderão estender sua aplicação do presente estudo a uma diversidade maior de topologias de redes, por exemplo, não apenas a backbones, mas também a redes metropolitanas. A métrica composta pode ser aperfeiçoada, por exemplo utilizando funções de utilidade não lineares, tal como sugerido ao final da Seção 3.4. Pode ainda recorrer a outras metodologias de auxílio multicritério a decisão (AMD).

Outra possível rota de evolução seria, além da investigação de vulnerabilidades, pensar em outros experimentos sobre as redes brasileiras, utilizando grafos valorados e coordenadas geográficas. Experimentos mais sofisticados poderiam ser realizados, envolvendo capacidade dos enlaces, roteamento ótimo e inibição geográfica, entre outros. Um modelo com uma rede brasileira de referência poderia ser de interesse para a SNDlib [8], a exemplo do que lá existe para vários países ou regiões (EUA, Europa, Índia etc.). Isto facilitaria a extensão dos experimentos a um nível internacional, além de possibilitar o uso de ferramentas disponíveis na SNDlib. Seria possível realizar, por exemplo, experimentos de tráfego dinâmico muito interessantes, comparando seus resultados com outras redes internacionais. Tais experimentos poderiam incluir estimativas de demanda, 
previsões de variação tráfego e estatísticas de população, entre outros, com grande riqueza de informações a analisar.

Desta forma, demonstra-se a utilidade do recurso a métricas estruturais e espectrais aplicadas a grafos que modelam as redes. Os métodos e ferramentas aqui utilizados se mostraram empiricamente válidos para os modelos de redes brasileiras estudados. Esses mesmos métodos e ferramentas podem, em princípio, ser aplicados a quaisquer redes de telecomunicações. Os modelos utilizados podem ser tão próximos à realidade quanto se queira. Os resultados podem ser úteis para se analisar possíveis aperfeiçoamentos na topologia das redes existentes, incluindo suas interconexões. Além disso, dão subsídios a estudos sobre a mitigação de efeitos em cascata resultantes de desastres naturais ou provocados pelo homem, bem como de ameaças físicas ou cibernéticas, com miras à proteção das infraestruturas críticas de telecomunicações. 


\section{REFERÊNCIAS BIBLIOGRÁFICAS}

[1] NAÇÕES UNIDAS - "Creation of a global culture of cybersecurity and the protection of critical information infrastructures". Res. 58/199. 2004. Disponível em: http://www.itu.int/ITU-D/cyb/cybersecurity/docs/UN_resolution_58_199.pdf

[2] HELLSTRÖM, T. - "Critical infrastructure and systemic vulnerability: Towards a planning framework," Safety Science, v. 45, n. 3, p. 415-430, Mar. 2007. Disponível em: http://linkinghub.elsevier.com/retrieve/pii/S0925753506000993

[3] GORMAN, S.; SCHINTLER, L.; KULKARNI, R.; STOUGH, R. - "The revenge of distance: vulnerability analysis of critical information infrastructure" School of Public Policy, George Mason University, 2004. Disponível em: http://arxiv.org/ftp/condmat/papers/0310/0310427.pdf

[4] MATISZIW, T.; MURRAY, A.; GRUBESIC, T. - "Exploring the vulnerability of network infrastructure to disruption," Annals of Regional Science, v. 43, n. 2, p. 307321, Abr. 2008. Disponível em: http://link.springer.com/10.1007/s00168-008-0235-X

[5] STROGATZ, S. - "Exploring complex networks" Nature-Insight Review Articles, Macmillan Publishers Ltd. V.410, p. 268-276, 2001. Disponível em: http://www.nature.com/nature/journal/v410/n6825/pdf/410268a0.pdf

[6] AMMANN, P.; WIJESEKERA, D.; KAUSHIK, S. - "Scalable, graph-based network vulnerability analysis," Proceedings of the 9th ACM conference on Computer and communications security - CCS '02, p. 217-234, 2002. Disponível em: http://d1.acm.org/citation.cfm?id=586110.586140

[7] ABREU, N.; DEL-VECCHIO, R.; TREVISAN, V.; VINAGRE, C. - "Teoria Espectral de Grafos - Uma Introdução". Minicurso apresentado no III Colóquio de Matemática da Região Sul. Florianópolis, Brasil, 2014. Disponível em: http://www.sbm.org.br/docs/coloquios/SU3-06.pdf

[8] SNDlib - "Library of test instances for Survivable fixed telecommunication Network Design". Zuse-Institute Berlin (ZIB). 2006. Disponível em: http://sndlib.zib.de/home.action

[9] STERBENZ, J.; CETINKAYA, K. ; HAMEED, A. ; JABBAR, A. ; ROHRER, P. "Modelling and Analysis of Network Resilience Communication Systems and 
Networks". Proceedings of the 3rd International Conference on Communication Systems and Networks - COMSNETS 2011, p.1-10, 2011.

[10] LIU, Y.; MENDIRATTA, V.; TRIVEDI, K. - "Survivability analysis of telephone access network" Proceedings of the 15th International Symposium on Software Reliability Engineering, IEEE Computer Society Washington. p. 367-378, 2004.

[11] NICOL, D.; SANDERS, W.; TRIVEDI, K. - "Model-based evaluation: From dependability to security" IEEE Transactions on Dependable and Secure Computing, v. 1, n. 1, p. 48-65, Jan. 2004.

[12] STERBENZ, J.; HUTCHISON, D.; ÇETINKAYA, K.; JABBAR, A., ROHRER, J.; SCHÖLLER, M.; SMITH, P. - "Resilience and survivability in communication networks: Strategies, principles, and survey of disciplines" Computer Networks, v. 54, n. $8, \quad$ p. 1245-1265, Jun. 2010 . Disponível em: http://linkinghub.elsevier.com/retrieve/pii/S1389128610000824

[13] PELLE, S. - "Géomatique: la théorie des graphes". Cours IT1 2002. École Nationale des Sciences Géographiques, Institut Géographique National, França, 2002. p. 12-65 Disponível em: http://pelle.stephane.free.fr/TheorieGraphes.pdf

[14] FREEMAN, L. - "Centrality in Social Networks Conceptual Clarification" Social Networks, Elsevier Sequoia S.A. v. 1, n. 3, p. 215-239, 1978.

[15] NEWMAN, M. - "A measure of betweenness centrality based on random walks," Social Networks, v. 27, n. 1, p. 39-54, 2005. Disponível em: http://arxiv.org/abs/cond$\underline{\text { mat } / 0309045}$

[16] TIZGHADAM, A.; LI, W.; LEON-GARCIA, A. - "Network criticality in vehicular networks," ACM SIGMETRICS Performance Evaluation Review, v. 40, n. 3, p. 107, 2012. Disponível em: http://dl.acm.org/citation.cfm?doid=2425248.2425278

[17] JOLLIFFE, I. - "Principal Component Analysis", Second Edition. Springer. 2002.

[18] SPIELMAN, D. - "Spectral Graph Theory", in Combinatorial Scientific Computing. CRC Press. Cap. 18, p. 495-524, 2012. Disponível em: http://www.cs.yale.edu/homes/spielman/PAPERS/SGTChapter.pdf

[19] SEARY A.; RICHARDS, W. - "Spectral methods for analyzing and visualizing networks: an introduction" Dynamic Social Networks Modeling and Analysis: Workshop Summary and Papers, National Academies Press, p. 1-20, 2003. Disponível em: http://www.sfu.ca/personal/archives/richards/Pages/NAS.AJS-WDR.pdf 
[20] SEARY A.; RICHARDS, W. - "Partitioning Networks by Eigenvectors". Proceedings of the International Conference on Social Networks, v., p. 47-58, 1995. Disponível em: http://www.sfu.ca/personal/archives/richards/Pages/london98.pdf

[21] ERDÖS, P.; RÉNYI, A. - “On Random Graphs I'. Publicationes Mathematicae 6, 1959, p. 290-297 Disponível em: http://snap.stanford.edu/class/cs224wreadings/erdos59random.pdf

[22] WATTS, D.; STROGATZ, S. - "Collective dynamics of small-world networks". Nature. Macmillan Publishers Ltd. v. 393, p. 440-442. 1998

[23] BARABÁSI, A.; ALBERT, R. - "Emergence of scaling in random networks". Science $\quad$ n. 286, p. 509-512, 1999. Disponível em: http://arxiv.org/pdf/condmat/9910332.pdf

[24] FAloutsos, M.; FAlOUTSOS, P.; FAlOUTSOS, C. - "On Power-Law Relationships of the Internet Topology". ACM SIGCOMM Computer Communication Review, v. 29, n. 5, p.251, out. 1999. Disponível em: http://snap.stanford.edu/class/cs224w-readings/faloutsos99powerlaw.pdf

[25] ZHAO, L; PARK, K.; LAI,Y. - "Attack vulnerability of scale-free networks due to cascading breakdown”. Physical Review E, v. 70, n. 3, p. 1-4, Sep. 2004.

[26] HALAPPANAVAR; CHOUDHURY, S.; HOGAN, E.; HUI, P.; JOHNSON, J., RAY, I.; HOLDER, L. - "Towards a Networks-of-Networks Framework for Cyber Security". Proceedings of the IEEE Intelligence and Security Informatics (ISI) 2013. Disponível em: http://arxiv.org/abs/1304.6761

[27] ALBERT, R.; JEONG, J.; BARABÁSI, A. - "Error and attack tolerance of complex networks". Nature. Macmillan Publishers Ltd. v. 406, p.378-382, Jul. 2000. Disponível em: http://www.barabasilab.com/pubs/CCNR-ALB_Publications/20000727 Nature-ErrorAttack/200007-27 Nature-ErrorAttack.pdf

[28] HOLME, P.; KIM, B.; YOON, C.; HAN, S. - "Attack vulnerability of complex networks", Physical Review E, v. 65, n. 5, p. 056109, May 2002.

[29] GRUBESIC T.; MURRAY, A. - "Vital nodes, interconnected infrastructures, and the geographies of network survivability". Annals of The Association of American Geographers, v. 96, n. 1, p. 64-83, 2006.

[30] TOME, S.; TRINDADE, M.; RIBEIRO, S.; CUCULO, C.; LAGE, L.; MARTINO, E.; SOUZA, R. - "Identificação de Infraestrutura Crítica de Telecomunicações com a Metodologia MI2C”. XXVII Simpósio Brasileiro de Telecomunicações - SBrT2009, Blumenau, Brasil, $2009 . \quad$ Set. Disponível em: 
http://www.eletrica.ufpr.br/anais/sbrt/SBrT27/Sess $\% \mathrm{C} 3 \% \mathrm{~B} 5 \mathrm{es} \% 20 \mathrm{~T} \% \mathrm{C} 3 \% \mathrm{~A} 9 \mathrm{cnicas}$ Artigos/Sess $\%$ C3\%A3o\%20T\%C3\%A9cnica 23/1 55899.pdf

[31] ANATEL - "Solução de suporte às atividades de gestão de risco relacionadas à segurança das infraestruturas críticas de telecomunicações e de monitoramento de redes, treinamento e garantia”. Contrato $n^{o}$. 002/2013 (SIEC). Disponível em: http://www.portaldatransparencia.gov.br/copa2014/gestor/download?idAnexo=31763

[32] MOTTA, M. - “Topologia dos backbones de internet no Brasil”, Revista Sociedade \& Natureza, Uberlândia, ano 24, n.1., p. 21-36, 2012. Disponível em: http://www.scielo.br/pdf/sn/v24n1/v24n1a03

[33] AlVES, N.; ALBUQUERQUE, M.; ALBUQUERQUE, M.; ASSIS, J. “Topologia e Modelagem Relacional da Internet Brasileira”. XXVI Iberian Latin American Congress on Computational Methods in Engineering - CILAMCE'05, Guarapari, Brasil, Out. 2005. Disponível em: http://mesonpi.cat.cbpf.br/redes/modelagem.pdf

[34] NEUMAYER, S.; ZUSSMAN, G.; COHEN, R.; MODIANO, E. - "Assessing the impact of geographically correlated network failures". Proceedings - IEEE Military Communication Conference-MILCOM, 2008.

[35] TRAJANOVSKI, S.; KUIPERS, F.; VAN MIEGHEM, P. - "Finding critical regions in a network". 2013 IEEE Conference on Computer Communications Workshops. (INFOCOM WKSHPS), p. 223-228, Apr. 2013.

[36] AGARWAL, P.; EFRAT, A.; GANJUGUNTE, S.; HAY, D. - "Network vulnerability to single, multiple, and probabilistic physical attacks". Proceedings IEEE Military Communications Conference MILCOM, p. 1824-1829, 2010.

[37] BEGUIN, H.; THOMAS, I. - "Morphologie du réseau de communication et localisations optimales d'activités. Quelle mesure pour exprimer la forme d'un réseau ?”. Cybergeo: Révue Européenne de Géographie, Systèmes, Modélisation, Géostatistiques, doc. 26, 1997. Disponível em: http://cybergeo.revues.org/2189

[38] FIEDLER, M. - "Algebraic connectivity of graphs". Czechoslovak Mathematical Journal. v.23, n. 2, p. 298-305, 1973. Disponível em: http://dml.cz/bitstream/handle/10338.dmlcz/101168/CzechMathJ_23-1973-2_11.pdf

[39] JAMAKOVIC, A.; UHLIG, S. - "On the relationship between the algebraic connectivity and graph's robustness to node and link failures". 2007 Next Generation Internet Networks, p. 96-102, May 2007. 
[40] TIZGHADAM, A; LEON-GARCIA, A. - "On Robust Traffic Engineering in Core Networks". IEEE “GLOBECOM” 2008 proceedings, p. 1-6. 2008

[41] TIZGHADAM A.; LEON-GARCIA, A. - "Autonomic traffic engineering for network robustness". IEEE Journal on Selected Areas in Communications, v. 28, n. 1, p. 39-50, 2010.

[42] FAY, D.; HADDADI, H.; THOMASON, A.; MOORE, A.; MORTIER, R.; JAMAKOVIC, A.; UHLIG, S.; RIO, M. - "Weighted spectral distribution for internet topology analysis: theory and applications”. IEEE/ACM Transactions on Networking, v.18, n. 1, p. 164-176, 2010.

[43] LONG, X.; TIPPER, D.; GOMES, T. - "Measuring the survivability of networks to geographic correlated failures”. Optical Switching and Networking, v.14, p. 117-133, 2014. Disponível em: http://linkinghub.elsevier.com/retrieve/pii/S1573427714000381

[44] LIU, W.; PAWLIKOWSKI, K.; SIRISENA, H. - "Algebraic connectivity metric for spare capacity allocation problem in survivable networks". Computer Communications. v.34, n.12, p. 1425-1435. 2011 Disponível em: http://linkinghub.elsevier.com/retrieve/pii/S014036641100003X

[45] BIGDELI, A.; TIZGHADAM, A.; LEON-GARCIA, A. - "Comparison of network criticality, algebraic connectivity, and other graph metrics". Proceedings of 1st Annual Workshop on Simplifying Complex Network for Practitioners, Veneza, Italia, 2009. Disponível em: http://individual.utoronto.ca/ali tizghadam/simplex.pdf

[46] KUROSE, J.; ROSS, K. - "Computer Networking - A Top-Down Approach”. 6a. edição. Pearson Education, 2012.

[47] GOMES, C. - "Principais Características da Teoria da Utilidade Multiatributo e Análise Comparativa com a Teoria da Modelagem de Preferências e Teoria das Expectativas". XVIII Encontro Nacional de Engenharia de Produção - Enegep 1998, Niterói, Brasil, $1998 . \quad$ Disponível em: http://www.abepro.org.br/biblioteca/enegep1998_art042.pdf

[48] SCHÄFER, R. - "Rules for Using Multi-Attribute Utility Theory for Estimating a User's Interests". Proceedings of the 9th German Society for Informatics (GI) Workshop: ABIS-Adaptivität und Benutzermodellierung in interaktiven Softwaresystemen, Dortmund, Germany. 2001. Disponível em: http://citeseerx.ist.psu.edu/viewdoc/download?doi=10.1.1.120.4425\&rep=rep1\&type $=p$ $\underline{\mathrm{df}}$ 
[49] IBGE - "Municípios com população residente superior a 50000 pessoas, em ordem decrescente de população residente - 2010". Sinopse do Censo Demográfico 2010, Instituto Brasileiro de Geografia e Estatística, 2014. Disponível em: http://www.ibge.gov.br/home/estatistica/populacao/censo2010/tabelas pdf/Brasil tab 1 1_. $14 . \mathrm{pdf}$

[50] CONVERGE COMUNICACOES - “Atlas Brasileiro de Telecomunicações”. São Paulo, 2014. Disponível em: http://issuu.com/telaviva/docs/atlas2014 site baix2

[51] ORLOWSKI, S.; WESSÄLY, R.; PIÓRO, M.; TOMASZEWSKI, A. . - "SNDlib 1.0-Survivable Network Design Library”. Networks, v. 55, n. 3, p. 276-286, 2010. Disponível em: https://opus4.kobv.de/opus4-zib/files/958/ZR-07-15.pdf

[52] MAESSCHALCK, S.; COLLE, D.; LIEVENS, I.; PICKAVET, M.; DEMEESTER P.; MAUZ, C.; JAEGER, M.; INKRET, R.; MIKAC, B.; DERKACZ, J. - "PanEuropean optical transport networks: An availability-based comparison". Photonic Network Communications, Springer, v. 5, n. 3, p. 203-225, Maio 2003. Disponível em: http://hdl.handle.net/1854/LU-287620

[53] TAPOLCAI, J. - "Routing Algorithms In Survivable Telecommunication Networks". PhD thesis, Department of Computer Science and Information Theory, University of Technology and Economics, Budapest, Hungria, 2005. Disponível em: http://www.omikk.bme.hu/collections/phd/Villamosmernoki_es_Informatikai_Kar/200 5/Tapolcai_Janos/tezis eng.pdf 


\section{APÊNDICES}




\section{Apêndice A - Dados}

\section{A.1 - Dados da rede Op_A}

\begin{tabular}{|c|c|c|}
\hline Nó & Cidade ou RM & Sigla \\
\hline 1 & São Paulo & SP \\
\hline 2 & Rio de Janeiro & RJ \\
\hline 3 & Belo Horizonte & $\mathrm{BH}$ \\
\hline 4 & Porto Alegre & PA \\
\hline 5 & Brasilia & BS \\
\hline 6 & Recife & $\mathrm{RE}$ \\
\hline 7 & Fortaleza & FO \\
\hline 8 & Salvador & SA \\
\hline 9 & Curitiba & CR \\
\hline 10 & Campinas & CA \\
\hline 11 & Belém & $\mathrm{BL}$ \\
\hline 12 & Goiânia & GO \\
\hline 13 & Manaus & $\mathrm{MN}$ \\
\hline 14 & Natal & NT \\
\hline 15 & São Luís & SL \\
\hline 16 & João Pessoa & $J P$ \\
\hline 17 & Maceió & $\mathrm{MO}$ \\
\hline 18 & Teresina & TE \\
\hline 19 & Joinville & JO \\
\hline 20 & Florianópolis & $\mathrm{FL}$ \\
\hline 21 & Aracaju & AJ \\
\hline 22 & Cuiabá & $\mathrm{CB}$ \\
\hline 23 & Juiz de Fora & $J F$ \\
\hline 24 & Campo Grande & CG \\
\hline 25 & Ribeirão Preto & RB \\
\hline 26 & Uberlândia & UI \\
\hline 27 & Palmas & $\mathrm{PL}$ \\
\hline 28 & Montes Claros & $\mathrm{MC}$ \\
\hline 29 & Umuarana & UM \\
\hline 30 & Ponta Grossa & PG \\
\hline 31 & Jundiaí & JU \\
\hline 32 & S.J.Rio Preto & $\mathrm{RP}$ \\
\hline 33 & Chapecó & $\mathrm{CH}$ \\
\hline 34 & Imperatriz & IM \\
\hline 35 & Bauru & $\mathrm{BU}$ \\
\hline 36 & Santa Maria & SM \\
\hline 37 & Pres. Prudente & PP \\
\hline 38 & Lages & LA \\
\hline 39 & Pato Branco & PB \\
\hline
\end{tabular}

\begin{tabular}{|c|c|c|}
\hline \multicolumn{2}{|c|}{ Enlace } & Sigla \\
\hline 1 & 2 & SP-RJ \\
\hline 1 & 3 & SP-BH \\
\hline 1 & 9 & SP-CR \\
\hline 1 & 30 & SP-PG \\
\hline 1 & 31 & SP-JU \\
\hline 1 & 35 & SP-BU \\
\hline 2 & 8 & RJ-SA \\
\hline 2 & 23 & RJ-JF \\
\hline 3 & 5 & BH-BS \\
\hline 3 & 8 & BH-SA \\
\hline 3 & 26 & BH-UI \\
\hline 3 & 23 & BH-JF \\
\hline 3 & 28 & BH-MC \\
\hline 4 & 20 & PA-FL \\
\hline 4 & 33 & PA-CH \\
\hline 4 & 36 & PA-SM \\
\hline 4 & 38 & PA-LA \\
\hline 17 & 21 & NT-JP \\
\hline 19 & 20 & SL-TE \\
\hline 22 & 24 & CB-AJ \\
\hline 24 & 29 & CG-UM \\
\hline 25 & 26 & RB-UI \\
\hline 27 & 32 & RB-RP \\
\hline 27 & 34 & PL-IM \\
\hline 29 & 30 & UM-PG \\
\hline 29 & 39 & UM-PB \\
\hline 30 & 38 & PG-LA \\
\hline 30 & 39 & PG-PB \\
\hline 32 & 37 & RP-PP \\
\hline 33 & 36 & CH-SM \\
\hline 33 & 38 & CH-LA \\
\hline 35 & 37 & BU-PP \\
\hline
\end{tabular}

\begin{tabular}{|c|c|c|}
\hline 5 & 12 & BS-GO \\
\hline 5 & 27 & BS-PL \\
\hline 6 & 7 & RE-FO \\
\hline 6 & 16 & RE-JP \\
\hline 6 & 17 & RE-MO \\
\hline 7 & 8 & FO-SA \\
\hline 7 & 14 & FO-NT \\
\hline 7 & 18 & FO-TE \\
\hline 8 & 21 & SA-AJ \\
\hline 8 & 28 & SA-MC \\
\hline 9 & 19 & CR-JO \\
\hline 9 & 30 & CR-PG \\
\hline 10 & 25 & CA-RB \\
\hline 10 & 31 & CA-JU \\
\hline 11 & 13 & BL-MN \\
\hline 11 & 15 & BL-SL \\
\hline 11 & 18 & BL-TE \\
\hline 11 & 34 & BL-IM \\
\hline 12 & 22 & GO-CB \\
\hline 12 & 24 & GO-CG \\
\hline 12 & 26 & GO-UI \\
\hline 13 & 34 & MN-IM \\
\hline
\end{tabular}


A.2 - Dados da rede Op_B

\begin{tabular}{|c|c|c|}
\hline Nó & Cidade ou RM & Sigla \\
\hline 1 & São Paulo & SP \\
\hline 2 & Rio de Janeiro & RJ \\
\hline 3 & Belo Horizonte & $\mathrm{BH}$ \\
\hline 4 & Porto Alegre & PA \\
\hline 5 & Brasília & BS \\
\hline 6 & Recife & $\mathrm{RE}$ \\
\hline 7 & Fortaleza & FO \\
\hline 8 & Salvador & SA \\
\hline 9 & Curitiba & $\mathrm{CR}$ \\
\hline 10 & Campinas & $\mathrm{CA}$ \\
\hline 11 & Belém & $\mathrm{BL}$ \\
\hline 12 & Goiânia & GO \\
\hline 13 & Manaus & $\mathrm{MN}$ \\
\hline 14 & Vitória & VI \\
\hline 15 & Natal & NT \\
\hline 16 & São Luís & SL \\
\hline 17 & João Pessoa & $J P$ \\
\hline 18 & Maceió & $\mathrm{MO}$ \\
\hline 19 & Teresina & $\mathrm{TE}$ \\
\hline 20 & Joinville & JO \\
\hline 21 & Londrina & LO \\
\hline 22 & Florianópolis & $\mathrm{FL}$ \\
\hline 23 & Aracaju & AJ \\
\hline 24 & Cuiabá & $\mathrm{CB}$ \\
\hline 25 & Campo Grande & CG \\
\hline 26 & Petrolina & PE \\
\hline 27 & Feira de Santana & FS \\
\hline 28 & Uberlândia & UI \\
\hline 29 & Palmas & $\mathrm{PL}$ \\
\hline 30 & Caxias do Sul & $C X$ \\
\hline 31 & Porto Velho & PV \\
\hline 32 & Chapecó & $\mathrm{CH}$ \\
\hline 33 & Crisciúma & $\mathrm{CS}$ \\
\hline 34 & Montes Claros & $\mathrm{MC}$ \\
\hline 35 & Imperatriz & IM \\
\hline 36 & Pelotas & PS \\
\hline 37 & Vit. da Conquista & VC \\
\hline 38 & Gov. Valadares & GV \\
\hline 39 & Santa Maria & SM \\
\hline 40 & Foz do Iguaçu & $\mathrm{FI}$ \\
\hline 41 & Macaé & $\mathrm{ME}$ \\
\hline
\end{tabular}

\begin{tabular}{|c|c|c|c|c|c|}
\hline \multicolumn{2}{|c|}{ Enlace } & Sigla & \multicolumn{2}{|c|}{ Enlace } & Sigla \\
\hline 1 & 2 & SP-RJ & 11 & 35 & BL-IM \\
\hline 1 & 3 & SP-BH & 12 & 24 & GO-CB \\
\hline 1 & 9 & SP-CR & 12 & 35 & GO-IM \\
\hline 1 & 10 & SP-CA & 13 & 31 & MN-PV \\
\hline 2 & 3 & RJ-BH & 14 & 38 & VI-GV \\
\hline 2 & 41 & RJ-ME & 14 & 41 & VI-ME \\
\hline 3 & 5 & $\mathrm{BH}-\mathrm{BS}$ & 15 & 17 & NT-JP \\
\hline 3 & 28 & $\mathrm{BH}-\mathrm{UI}$ & 16 & 19 & SL-TE \\
\hline 3 & 34 & BH-MC & 18 & 23 & MO-AJ \\
\hline 3 & 38 & BH-GV & 20 & 22 & JO-FL \\
\hline 4 & 30 & PA-CX & 21 & 25 & LO-CG \\
\hline 4 & 33 & PA-CS & 21 & 40 & LO-FI \\
\hline 4 & 36 & PA-PS & 22 & 32 & FL-CS \\
\hline 4 & 39 & PA-SM & 22 & 33 & $\mathrm{FL}-\mathrm{CH}$ \\
\hline 5 & 17 & BS-GO & 24 & 25 & CB-CG \\
\hline 5 & 28 & BS-UI & 24 & 31 & CB-PV \\
\hline 5 & 29 & BS-PL & 26 & 27 & PE-FS \\
\hline 6 & 17 & RE-JP & 29 & 35 & PA-IM \\
\hline 6 & 18 & RE-MO & 30 & 32 & $\mathrm{CX}-\mathrm{CH}$ \\
\hline 6 & 19 & RE-TE & 30 & 39 & CX-SM \\
\hline 6 & 26 & RE-PE & 32 & 40 & $\mathrm{CH}-\mathrm{FI}$ \\
\hline 7 & 8 & FO-SA & 36 & 39 & PS-SM \\
\hline 7 & 11 & FO-BL & 37 & 38 & VC-GV \\
\hline
\end{tabular}

\begin{tabular}{|c|c|c|}
\hline 7 & 13 & FO-MN \\
\hline 7 & 15 & FO-NT \\
\hline 7 & 16 & FO-SL \\
\hline 7 & 19 & FO-TE \\
\hline 8 & 14 & SA-AJ \\
\hline 8 & 23 & SA-VI \\
\hline 8 & 27 & SA-FS \\
\hline 8 & 34 & SA-MC \\
\hline 8 & 37 & SA-VC \\
\hline 9 & 20 & CR-JO \\
\hline 9 & 21 & CR-LO \\
\hline 9 & 22 & CR-FL \\
\hline 9 & 40 & CR-FI \\
\hline 10 & 28 & CA-UI \\
\hline 11 & 16 & BL-SL \\
\hline 11 & 19 & BL-TE \\
\hline
\end{tabular}


A.3 - Dados da rede Op_C

\begin{tabular}{|c|c|c|}
\hline Nó & Cidade ou RM & Sigla \\
\hline 1 & São Paulo & $\mathrm{SP}$ \\
\hline 2 & Rio de Janeiro & $\mathrm{RJ}$ \\
\hline 3 & Belo Horizonte & $\mathrm{BH}$ \\
\hline 4 & Porto Alegre & $\mathrm{PA}$ \\
\hline 5 & Brasilia & $\mathrm{BS}$ \\
\hline 6 & Recife & $\mathrm{RE}$ \\
\hline 7 & Salvador & $\mathrm{SA}$ \\
\hline 8 & Fortaleza & $\mathrm{FO}$ \\
\hline 9 & Curitiba & $\mathrm{CR}$ \\
\hline 10 & Campinas & $\mathrm{CA}$ \\
\hline 11 & Belém & $\mathrm{BL}$ \\
\hline 12 & Goiânia & $\mathrm{GO}$ \\
\hline 13 & Manaus & $\mathrm{MN}$ \\
\hline 14 & Vitória & $\mathrm{VI}$ \\
\hline 15 & Baixada Santista & $\mathrm{SN}$ \\
\hline 16 & Natal & $\mathrm{NT}$ \\
\hline 17 & São Luís & $\mathrm{SL}$ \\
\hline 18 & João Pessoa & $\mathrm{JP}$ \\
\hline 19 & Maceí́ & $\mathrm{MO}$ \\
\hline 20 & Teresina & $\mathrm{TE}$ \\
\hline 21 & Joinville & $\mathrm{JO}$ \\
\hline 22 & Londrina & $\mathrm{LO}$ \\
\hline 23 & Florianópolis & $\mathrm{FL}$ \\
\hline 24 & Aracaju & $\mathrm{AJ}$ \\
\hline 25 & Cuiabá & $\mathrm{CB}$ \\
\hline 26 & Juiz de Fiora & $\mathrm{JF}$ \\
\hline 27 & Campo Grande & $\mathrm{CG}$ \\
\hline 28 & Petrolina & $\mathrm{PE}$ \\
\hline 29 & Ribeirão Preto & $\mathrm{RB}$ \\
\hline 30 & Uberlândia & $\mathrm{UI}$ \\
\hline 31 & Palmas & $\mathrm{PL}$ \\
\hline 32 & Caxias do Sul & $\mathrm{CX}$ \\
\hline 33 & Porto Velho & $\mathrm{PV}$ \\
\hline 34 & Campina Grande & $\mathrm{CP}$ \\
\hline 35 & Chapecó & $\mathrm{CH}$ \\
\hline 36 & Imperatriz & $\mathrm{IM}$ \\
\hline 37 & Bauru & $\mathrm{BU}$ \\
\hline 38 & Pelotas & $\mathrm{PE}$ \\
\hline 39 & Vit. da Conquista & $\mathrm{VC}$ \\
\hline 40 & Gov. Valadares & $\mathrm{GV}$ \\
\hline 41 & Santa Maria & $\mathrm{SM}$ \\
\hline 42 & Foz do Iguaçu & $\mathrm{FI}$ \\
\hline 43 & Macaé & $\mathrm{ME}$ \\
\hline 44 & Dourados & $\mathrm{DO}$ \\
\hline 45 & Uruguaiana & $\mathrm{UR}$ \\
\hline 47 & Varginha & $\mathrm{VA}$ \\
\hline & Itumbiara & $\mathrm{IT}$ \\
\hline
\end{tabular}

\begin{tabular}{|c|c|c|}
\hline \multicolumn{2}{|c|}{ Enlace } & Sigla \\
\hline 1 & 2 & SP-RJ \\
\hline 1 & 10 & SP-CA \\
\hline 1 & 9 & SP-CR \\
\hline 1 & 15 & SP-SN \\
\hline 1 & 46 & SP-VA \\
\hline 2 & 15 & RJ-SN \\
\hline 2 & 26 & RJ-JF \\
\hline 2 & 43 & RJ-ME \\
\hline 3 & 5 & BH-BS \\
\hline 3 & 26 & BH-JF \\
\hline 3 & 40 & BH-GV \\
\hline 3 & 46 & BH-VA \\
\hline 3 & 47 & BH-UI \\
\hline 4 & 23 & PA-FL \\
\hline 4 & 32 & PA-CX \\
\hline 4 & 38 & PA-PL \\
\hline 4 & 41 & PA-SM \\
\hline 5 & 12 & BS-GO \\
\hline 5 & 47 & BS-IT \\
\hline 6 & 18 & RE-JP \\
\hline 6 & 19 & RE-MO \\
\hline 6 & 34 & RE-CP \\
\hline 7 & 14 & SA-VI \\
\hline 7 & 24 & SA-AJ \\
\hline 7 & 28 & SA-PE \\
\hline 7 & 39 & SA-VC \\
\hline 8 & 16 & FO-NT \\
\hline 8 & 20 & FO-TE \\
\hline 8 & 28 & FO-PE \\
\hline 8 & 34 & FO-CP \\
\hline 9 & 21 & CR-JO \\
\hline 9 & 22 & CR-LO \\
\hline 9 & 23 & CR-FL \\
\hline 10 & 29 & CA-RB \\
\hline
\end{tabular}

\begin{tabular}{|c|c|c|}
\hline \multicolumn{2}{|c|}{ Enlace } & Sigla \\
\hline 11 & 17 & BL-SL \\
\hline 11 & 20 & BL-TE \\
\hline 11 & 36 & BL-IM \\
\hline 11 & 17 & BL-SL \\
\hline 11 & 20 & BL-TE \\
\hline 12 & 25 & GO-CB \\
\hline 12 & 31 & GO-PL \\
\hline 13 & 33 & MN-PV \\
\hline 13 & 36 & MN-IM \\
\hline 14 & 40 & VI-GV \\
\hline 14 & 43 & VI-ME \\
\hline 16 & 18 & NT-JP \\
\hline 17 & 36 & SL-IM \\
\hline 19 & 24 & MO-AJ \\
\hline 21 & 32 & JO-CX \\
\hline 22 & 42 & LO-FI \\
\hline 22 & 37 & LO-BU \\
\hline 25 & 27 & CB-CG \\
\hline 25 & 33 & CB-PV \\
\hline 27 & 44 & CG-DO \\
\hline 29 & 30 & RB-UI \\
\hline 29 & 37 & RB-BU \\
\hline 30 & 47 & UI-IT \\
\hline 31 & 36 & PL-IM \\
\hline 35 & 41 & CH-SM \\
\hline 35 & 42 & CH-FI \\
\hline 37 & 44 & BU-DO \\
\hline 38 & 45 & PL-UR \\
\hline 39 & 40 & VC-GV \\
\hline 41 & 45 & SM-UR \\
\hline & & \\
\hline
\end{tabular}




\section{A.4 - Dados da rede Op_D}

\begin{tabular}{|c|c|c|}
\hline Nó & Cidade ou RM & Sigla \\
\hline 1 & São Paulo & SP \\
\hline 2 & Rio de Janeiro & RJ \\
\hline 3 & Belo Horizonte & BH \\
\hline 4 & Porto Alegre & PA \\
\hline 5 & Brasilia & BS \\
\hline 6 & Recife & RE \\
\hline 7 & Fortaleza & FO \\
\hline 8 & Salvador & SA \\
\hline 9 & Curitiba & CR \\
\hline 10 & Campinas & CA \\
\hline 11 & Belém & BL \\
\hline 12 & Goiânia & GO \\
\hline 13 & Manaus & MN \\
\hline 14 & Vitória & VI \\
\hline 15 & Natal & NT \\
\hline 16 & São Luís & SL \\
\hline 17 & João Pessoa & JP \\
\hline 18 & Maceió & MO \\
\hline 19 & Teresina & TE \\
\hline 20 & Londrina & LO \\
\hline 21 & Florianópolis & FL \\
\hline 22 & Aracaju & AJ \\
\hline 23 & Cuiabá & CB \\
\hline 24 & Campo Grande & CG \\
\hline 25 & Ribeirão Preto & RB \\
\hline 26 & Uberlândia & UI \\
\hline 27 & Palmas & PL \\
\hline 28 & Porto Velho & PV \\
\hline 29 & Imperatriz & IM \\
\hline 30 & Bauru & BU \\
\hline 31 & Pelotas & PS \\
\hline 32 & Santa Maria & SM \\
\hline 33 & Foz do Iguaçu & FI \\
\hline 34 & Passo Fundo & PF \\
\hline 35 & Uruguaiana & UR \\
\hline 36 & Itumbiara & IT \\
\hline
\end{tabular}

\begin{tabular}{|c|c|c|}
\hline \multicolumn{2}{|c|}{ Enlace } & Sigla \\
\hline 1 & 2 & SP-RJ \\
\hline 1 & 3 & SP-BH \\
\hline 1 & 9 & SP-CR \\
\hline 1 & 10 & SP-CA \\
\hline 1 & 30 & SP-BU \\
\hline 2 & 3 & RJ-BH \\
\hline 2 & 14 & RJ-VI \\
\hline 3 & 26 & BH-UI \\
\hline 4 & 21 & PA-FL \\
\hline 4 & 34 & PA-PF \\
\hline 4 & 31 & PA-PS \\
\hline 4 & 32 & PA-SM \\
\hline 5 & 8 & BS-SA \\
\hline 5 & 12 & BS-GO \\
\hline 5 & 27 & BS-PL \\
\hline 6 & 17 & RE-JP \\
\hline 6 & 18 & RE-MO \\
\hline 7 & 8 & FO-SA \\
\hline 7 & 15 & FO-NT \\
\hline 7 & 19 & FO-TE \\
\hline 8 & 14 & SA-VI \\
\hline 8 & 22 & SA-AJ \\
\hline 9 & 20 & CR-LO \\
\hline 9 & 21 & CR-FL \\
\hline 10 & 25 & CA-RB \\
\hline 10 & 30 & CA-BU \\
\hline 11 & 13 & BL-MN \\
\hline 11 & 29 & BL-IM \\
\hline 12 & 36 & GO-IT \\
\hline 13 & 28 & MN-PV \\
\hline & & \\
\hline
\end{tabular}

\begin{tabular}{|l|l|c|}
\hline \multicolumn{2}{|c|}{ Enlace } & Sigla \\
\hline 15 & 17 & NT-JP \\
\hline 16 & 19 & SL-TE \\
\hline 16 & 29 & SL-IM \\
\hline 18 & 22 & MO-AJ \\
\hline 20 & 24 & LO-CG \\
\hline 20 & 30 & LO-BU \\
\hline 20 & 33 & LO-FI \\
\hline 23 & 28 & CB-PV \\
\hline 23 & 36 & CB-IT \\
\hline 24 & 30 & CG-BU \\
\hline 25 & 26 & RB-UI \\
\hline 26 & 36 & UI-IT \\
\hline 27 & 29 & PL-IM \\
\hline 31 & 32 & PS-SM \\
\hline 32 & 35 & SM-UR \\
\hline 33 & 34 & FI-PF \\
\hline 34 & 35 & PF-UR \\
\hline
\end{tabular}


A.5 - Dados da rede Op_E

\begin{tabular}{|c|c|c|}
\hline Nó & Cidade ou RM & Sigla \\
\hline 1 & São Paulo & SP \\
\hline 2 & Rio de Janeiro & RJ \\
\hline 3 & Belo Horizonte & $\mathrm{BH}$ \\
\hline 4 & Porto Alegre & PA \\
\hline 5 & Brasília & BS \\
\hline 6 & Recife & RE \\
\hline 7 & Fortaleza & FO \\
\hline 8 & Salvador & SA \\
\hline 9 & Curitiba & $\mathrm{CR}$ \\
\hline 10 & Campinas & CA \\
\hline 11 & Belém & $\mathrm{BL}$ \\
\hline 12 & Vale do Paraiba & SJ \\
\hline 13 & Goiânia & GO \\
\hline 14 & Manaus & $\mathrm{MN}$ \\
\hline 15 & Vitória & VI \\
\hline 16 & Baixada Santista & SN \\
\hline 17 & Natal & NT \\
\hline 18 & São Luís & SL \\
\hline 19 & João Pessoa & JP \\
\hline 20 & Maceió & $\mathrm{MO}$ \\
\hline 21 & Joinville & JO \\
\hline 22 & Londrina & LO \\
\hline 23 & Florianópolis & $\mathrm{FL}$ \\
\hline 24 & Aracaju & AJ \\
\hline 25 & Sorocaba & SO \\
\hline 26 & Cuiabá & $\mathrm{CB}$ \\
\hline 27 & Campo Grande & CG \\
\hline 28 & Ribeirão Preto & RB \\
\hline 29 & Palmas & $\mathrm{PL}$ \\
\hline 30 & Caxias do Sul & $C X$ \\
\hline 31 & S.J.Rio Preto & $\mathrm{RP}$ \\
\hline 32 & Porto Velho & PV \\
\hline 33 & Campina Grande & $\mathrm{CP}$ \\
\hline 34 & Chapecó & $\mathrm{CH}$ \\
\hline 35 & Bauru & $\mathrm{BU}$ \\
\hline 36 & Gov. Valadares & GV \\
\hline 37 & Santa Maria & SM \\
\hline 38 & Foz do Iguaçu & $\mathrm{FI}$ \\
\hline 39 & Presidente Prudente & PP \\
\hline 40 & Dourados & DO \\
\hline
\end{tabular}

\begin{tabular}{|c|c|c|}
\hline \multicolumn{2}{|c|}{ Enlace } & Sigla \\
\hline 1 & 3 & SP-BH \\
\hline 1 & 9 & SP-CR \\
\hline 1 & 10 & SP-CA \\
\hline 1 & 12 & SP-SJ \\
\hline 1 & 16 & SP-SN \\
\hline 1 & 25 & SP-SO \\
\hline 2 & 3 & RJ-BH \\
\hline 2 & 12 & RJ-SJ \\
\hline 2 & 15 & RJ-VI \\
\hline 3 & 5 & BH-BS \\
\hline 3 & 36 & BH-GV \\
\hline 4 & 23 & PA-FL \\
\hline 4 & 30 & PA-CX \\
\hline 4 & 37 & PA-SM \\
\hline 5 & 13 & BS-GO \\
\hline 5 & 28 & BS-RB \\
\hline 22 & 35 & Sigla \\
\hline 25 & 35 & LO-CX \\
\hline 26 & 27 & LO-FI \\
\hline 27 & 32 & CB-CG \\
\hline 28 & 40 & CG-DV \\
\hline 28 & 31 & RB-RP \\
\hline 30 & 34 & RB-BU \\
\hline 31 & 39 & RP-CH \\
\hline 34 & 37 & CH-SM \\
\hline 34 & 40 & CH-FI \\
\hline 35 & 39 & BU-PP \\
\hline
\end{tabular}

\begin{tabular}{|c|c|c|}
\hline 5 & 28 & BS-RB \\
\hline 5 & 29 & BS-PL \\
\hline 6 & 19 & RE-JP \\
\hline 6 & 20 & RE-MO \\
\hline 6 & 33 & RE-CP \\
\hline 7 & 17 & FO-NT \\
\hline 7 & 18 & FO-SL \\
\hline 7 & 33 & FO-CP \\
\hline 8 & 15 & SA-VI \\
\hline 8 & 24 & SA-AJ \\
\hline 8 & 36 & SA-GV \\
\hline 9 & 21 & CR-JO \\
\hline 9 & 22 & CR-LO \\
\hline 9 & 23 & CR-FL \\
\hline 9 & 38 & CR-FI \\
\hline 10 & 28 & CA-RB \\
\hline 11 & 14 & BL-MN \\
\hline 11 & 18 & BL-SL \\
\hline 11 & 29 & BL-PL \\
\hline 12 & 16 & SJ-SN \\
\hline 13 & 26 & GO-CB \\
\hline 14 & 32 & MN-PV \\
\hline 17 & 19 & NT-JP \\
\hline 20 & 24 & MO-AJ \\
\hline & & \\
\hline
\end{tabular}


A.6 - Dados da rede Cost266

\begin{tabular}{|c|c|c|}
\hline Nó & Cidade ou RM & Sigla \\
\hline 1 & Londres & LD \\
\hline 2 & Berlim & BL \\
\hline 3 & Madri & MD \\
\hline 4 & Roma & RM \\
\hline 5 & Atenas & AT \\
\hline 6 & Paris & PR \\
\hline 7 & Hamburgo & HB \\
\hline 8 & Budapeste & BP \\
\hline 9 & Varsóvia & WS \\
\hline 10 & Viena & VN \\
\hline 11 & Barcelona & BN \\
\hline 12 & Zurique & ZC \\
\hline 13 & Sófia & SF \\
\hline 14 & Munique & MC \\
\hline 15 & Mião & ML \\
\hline 16 & Praga & PG \\
\hline 17 & Belgrado & BG \\
\hline 18 & Lyon & LN \\
\hline 19 & Bruxelas & BS \\
\hline 20 & Birmingham & BH \\
\hline 21 & Marselha & MS \\
\hline 22 & Estocolmo & SH \\
\hline 23 & Zagreb & ZR \\
\hline 24 & Cracóvia & KR \\
\hline 25 & Amsterdam & AD \\
\hline 26 & Sevilha & SV \\
\hline 27 & Frankfurt & FK \\
\hline 28 & Palermo & PL \\
\hline 29 & Oslo & OS \\
\hline 30 & Helsinque & HS \\
\hline 31 & Glasgow & GG \\
\hline 32 & Dusseldorf & DD \\
\hline 33 & Copenhagem & CG \\
\hline 34 & Dublin & DB \\
\hline 35 & Lisboa & LB \\
\hline 36 & Bordeaux & BD \\
\hline 37 & Estrasburgo & SB \\
\hline & & \\
\hline
\end{tabular}

\begin{tabular}{|c|c|c|}
\hline \multicolumn{2}{|c|}{ Enlace } & Sigla \\
\hline 1 & 6 & LD-PR \\
\hline 1 & 20 & LD-BH \\
\hline 1 & 25 & LD-AD \\
\hline 1 & 34 & LD-DB \\
\hline 1 & 35 & LD-LB \\
\hline 2 & 7 & BL-HB \\
\hline 2 & 9 & BL-WS \\
\hline 2 & 14 & BL-MC \\
\hline 2 & 16 & BL-PG \\
\hline 2 & 33 & BL-CG \\
\hline 3 & 11 & MD-BN \\
\hline 3 & 35 & MD-LB \\
\hline 3 & 36 & MD-BD \\
\hline 4 & 15 & RM-ML \\
\hline 4 & 21 & RM-MS \\
\hline 4 & 23 & RM-ZR \\
\hline 4 & 28 & RM-PL \\
\hline 5 & 13 & AT-SF \\
\hline 5 & 23 & AT-ZR \\
\hline 5 & 28 & AT-PL \\
\hline 6 & 18 & ZC-LN \\
\hline 14 & ZC-SB \\
\hline 14 & 27 & SF-BG \\
\hline 17 & 23 & MG-IG \\
\hline 18 & 21 & LN-MS \\
\hline 19 & 25 & BS-AD \\
\hline 19 & 32 & BS-DD \\
\hline 20 & 31 & BH-GG \\
\hline 21 & 36 & MS-BD \\
\hline 22 & 30 & SH-HS \\
\hline 22 & 33 & SH-CG \\
\hline 25 & 31 & AD-GG \\
\hline 26 & 35 & SV-LB \\
\hline 27 & 32 & FK-DD \\
\hline 27 & 37 & FK-SB \\
\hline 29 & 30 & OS-HS \\
\hline 29 & 33 & OS-CG \\
\hline 31 & 34 & GG-DB \\
\hline
\end{tabular}


A.7 - Dados da rede Janos US-CA

\begin{tabular}{|c|c|c|}
\hline Nó & Cidade ou RM & Sigla \\
\hline 1 & New York & NY \\
\hline 2 & Los Angeles & LA \\
\hline 3 & Chicago & $\mathrm{CC}$ \\
\hline 4 & Dallas & $\mathrm{DL}$ \\
\hline 5 & Philadelphia & PD \\
\hline 6 & Houston & $\mathrm{HS}$ \\
\hline 7 & Toronto & TT \\
\hline 8 & Washington & WD \\
\hline 9 & Miami & $\mathrm{MM}$ \\
\hline 10 & Atlanta & AT \\
\hline 11 & Boston & BT \\
\hline 12 & San Francisco & SF \\
\hline 13 & Detroit & DT \\
\hline 14 & Phoenix & PN \\
\hline 15 & Seatle & ST \\
\hline 16 & Montreal & $\mathrm{MR}$ \\
\hline 17 & Minneapolis & $\mathrm{MP}$ \\
\hline 18 & San Diego & SD \\
\hline 19 & Saint Louis & SL \\
\hline 20 & Tampa & TP \\
\hline 21 & Denver & DV \\
\hline 22 & Pittsburgh & PB \\
\hline 23 & Vancouver & VC \\
\hline 24 & Portland & $\mathrm{PL}$ \\
\hline 25 & Sacramento & SM \\
\hline 26 & Cincinnati & $\mathrm{CN}$ \\
\hline 27 & Cleveland & $\mathrm{CL}$ \\
\hline 28 & Kansas City & $\mathrm{KC}$ \\
\hline 29 & Las Vegas & LV \\
\hline 30 & Indianapolis & IP \\
\hline 31 & Nashville & NV \\
\hline 32 & Memphis & $\mathrm{MH}$ \\
\hline 33 & Oklahoma City & $\mathrm{OH}$ \\
\hline 34 & Calgary & CG \\
\hline 35 & New Orleans & NO \\
\hline 36 & Salt Lake City & SC \\
\hline 37 & El Paso & $E P$ \\
\hline 38 & Winnipeg & WP \\
\hline 39 & Charleston & CR \\
\hline
\end{tabular}

\begin{tabular}{|c|c|c|}
\hline \multicolumn{2}{|c|}{ Enlace } & Sigla \\
\hline 1 & 5 & NY-PD \\
\hline 1 & 7 & NY-TT \\
\hline 1 & 11 & NY-BT \\
\hline 1 & 27 & NY-CL \\
\hline 2 & 12 & LA-SF \\
\hline 2 & 18 & LA-SD \\
\hline 2 & 29 & LA-LV \\
\hline 3 & 13 & CC-DT \\
\hline 3 & 17 & CC-MP \\
\hline 3 & 19 & CC-SL \\
\hline 3 & 30 & CC-IP \\
\hline 4 & 6 & DL-HS \\
\hline 4 & 21 & DL-DV \\
\hline 4 & 32 & DL-MH \\
\hline 4 & 33 & DL-OH \\
\hline 4 & 37 & DL-EP \\
\hline 5 & 8 & PD-WD \\
\hline 6 & 35 & HS-NO \\
\hline 6 & 37 & HS-EP \\
\hline 7 & 13 & TT-DT \\
\hline 7 & 16 & TT-MR \\
\hline 8 & 22 & WD-PB \\
\hline 8 & 39 & WD-CR \\
\hline 9 & 20 & MM-TP \\
\hline 9 & 35 & MM-NO \\
\hline 10 & 20 & AT-TP \\
\hline 10 & 31 & AT-NV \\
\hline 10 & 35 & AT-NO \\
\hline 10 & 39 & AT-CR \\
\hline 11 & 16 & BT-MR \\
\hline 12 & 24 & SF-PL \\
\hline 12 & 25 & SF-SM \\
\hline 13 & 27 & DT-CL \\
\hline 14 & 18 & PN-SD \\
\hline 14 & 29 & PN-LV \\
\hline 14 & 37 & PN-EP \\
\hline 15 & 23 & ST-VC \\
\hline 15 & 24 & ST-PL \\
\hline 17 & 28 & MP-KC \\
\hline & & \\
\hline
\end{tabular}

\begin{tabular}{|c|c|c|}
\hline \multicolumn{2}{|c|}{ Enlace } & Sigla \\
\hline 17 & 38 & MP-WP \\
\hline 19 & 28 & SL-KC \\
\hline 19 & 30 & SL-IP \\
\hline 19 & 32 & SL-MH \\
\hline 20 & 39 & TP-CR \\
\hline 21 & 28 & DV-KC \\
\hline 21 & 36 & DV-SC \\
\hline 22 & 27 & PB-CL \\
\hline 23 & 34 & VC-CG \\
\hline 24 & 36 & PL-SC \\
\hline 25 & 29 & SM-LV \\
\hline 25 & 36 & SM-SC \\
\hline 26 & 27 & CN-CL \\
\hline 26 & 30 & CN-IP \\
\hline 28 & 33 & KC-OH \\
\hline 29 & 36 & LV-SC \\
\hline 30 & 31 & IP-NV \\
\hline 31 & 32 & NV-MH \\
\hline 31 & 39 & NV-CR \\
\hline 32 & 35 & MH-NO \\
\hline 34 & 36 & CG-SC \\
\hline 34 & 38 & CG-WP \\
\hline
\end{tabular}




\section{Apêndice B - Medidas obtidas}

\section{B.1 - Retirada dos nós da rede Op_A}

\begin{tabular}{|c|c|c|c|c|c|c|c|c|c|c|c|c|c|c|c|c|c|c|c|c|c|c|c|c|c|c|c|c|c|c|c|c|c|c|c|c|c|c|c|}
\hline Métrica & SP & RJ & $3 \mathrm{H}$ & PA & BS & $\mathrm{RE}$ & FO & SA & $\mathrm{CR}$ & $\mathrm{CA}$ & $\mathrm{BL}$ & GO & $\mathrm{MN}$ & NT & $S L$ & $\mathrm{JP}$ & $\mathrm{MO}$ & $\mathrm{TE}$ & JO & $\mathrm{FL}$ & AJ & $\mathrm{CB}$ & $\mathrm{JF}$ & CG & $\mathrm{RB}$ & UI & $P L$ & $M C$ & UM & PG & JU & $\mathrm{RP}$ & $\mathrm{CH}$ & $\mathrm{IM}$ & $\mathrm{BU}$ & SM & PP & LA & PB \\
\hline ASI & & & 2,3 & 2,0 & 2,2 & 2,0 & 2,3 & 2,6 & 2 & 2,0 & 2,1 & 2,1 & 2,0 & 2,0 & 2,0 & 2,0 & 2,0 & 2,1 & 2,0 & 2,0 & 2,1 & , & 2,0 & 2,1 & 2,1 & 2,1 & 2,1 & 2,1 & 2,1 & 2,3 & 2,1 & 2,0 & 2,0 & 2,1 & 2,1 & 2,0 & 2,0 & 2,1 & 2,0 \\
\hline$N$ & 3,4 & 21 & 24 & 2,1 & 25 & 2,1 & 2,8 & 34 & 23 & 2 & -7 & (2) & 19 & 7 & 1,9 & 1,9 & 2 & 2,3 & 2,1 & 2,0 & 20 & 19 & 1,9 & 1 & ,2 & 2,1 & 3 & 1,9 & 2,1 & 2,9 & 0 & 2,0 & 1,9 & ,2 & 2,0 & 1,9 & 2,0 & 3 & 1,9 \\
\hline $1 / A D$ & 0 & 0, & 0,4 & 0,4 & 0,4 & 0,4 & 0,4 & 0 & 0 & 0,4 & 0,4 & 0,4 & 0,4 & 0,4 & 0,4 & 0,4 & 0,4 & 0,4 & 0,4 & 0,4 & 0,4 & 0,4 & 0,4 & 0,4 & 0,4 & 0,4 & 0,4 & 0,4 & 0,4 & 0,4 & 0,4 & 0,4 & 0,4 & 0,4 & 0,4 & 0,4 & 0,4 & 0,4 & 0,4 \\
\hline $1 / A C$ & 25 & 12 & 14 & 9 & 17 & 10 & 16 & 33 & 14 & 10 & 10 & 13 & 9 & 10 & 9 & 9 & 10 & 12 & 11 & 10 & 11 & 10 & 10 & 12 & 10 & 11 & 15 & 10 & 11 & 20 & 10 & 10 & 9 & 13 & 10 & 9 & 10 & 14 & 10 \\
\hline DI & + & 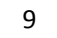 & 1 & 9 & 1 & 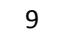 & + & 14 & 10 & 9 & 9 & 9 & 9 & 9 & 9 & 9 & 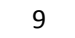 & 10 & 9 & 9 & 9 & 9 & 9 & 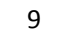 & r & 9 & 10 & 9 & 9 & 11 & 9 & 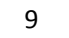 & 0 & 10 & 9 & 8 & 9 & 10 & 9 \\
\hline WS4 & 9 & 8,0 & 8,7 & 8,9 & 8,3 & 8,4 & 8,8 & 8,8 & 8,4 & 7,9 & 9,0 & 8,6 & 8,1 & 7,9 & 8,1 & 7,8 & 8,0 & 8,4 & 7,9 & 8,0 & 8,0 & 8,0 & 7,9 & 8,2 & 8,5 & 8,4 & 8,0 & 8,1 & 8,2 & 9,0 & 8,1 & 8,1 & 8,2 & 8,3 & 8,2 & 8,0 & 7,8 & 8,3 & 8,0 \\
\hline$S \times 100$ & 1,7 & 0 & 0 , & 0 , & 0,3 & 0,3 & 0,3 & 1,4 & 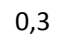 & 0,2 & 0,1 & 0,0 & 0,3 & 0,3 & 0,2 & - & 0,3 & 0 , & 0,2 & 0,3 & 0,2 & 0,2 & 0,1 & 0,1 & 0,2 & 0,0 & 0,3 & 0,1 & 0,0 & 0,1 & 0,1 & 0,3 & ,3 & ,4 & 0,0 & 0,2 & 0,2 & ,4 & ,2 \\
\hline 1 & 0,5 & ,6 & , & 0 & , & 6 & 0,0 & 0,0 & 0,0 & 0,6 & 0,6 & 0,5 & 0,0 & 0,0 & , & ( & 0,6 & 0,7 & 0,6 & 0,6 & 0,0 & 0,0 & 0,0 & 0,6 & 0,6 & 0,0 & 0,0 & 0,6 & 0,6 & 0,6 & 0,6 & 0,6 & 0,6 & 0,7 & 0,6 & 0,5 & 0,6 & 0,6 & 0,6 \\
\hline
\end{tabular}

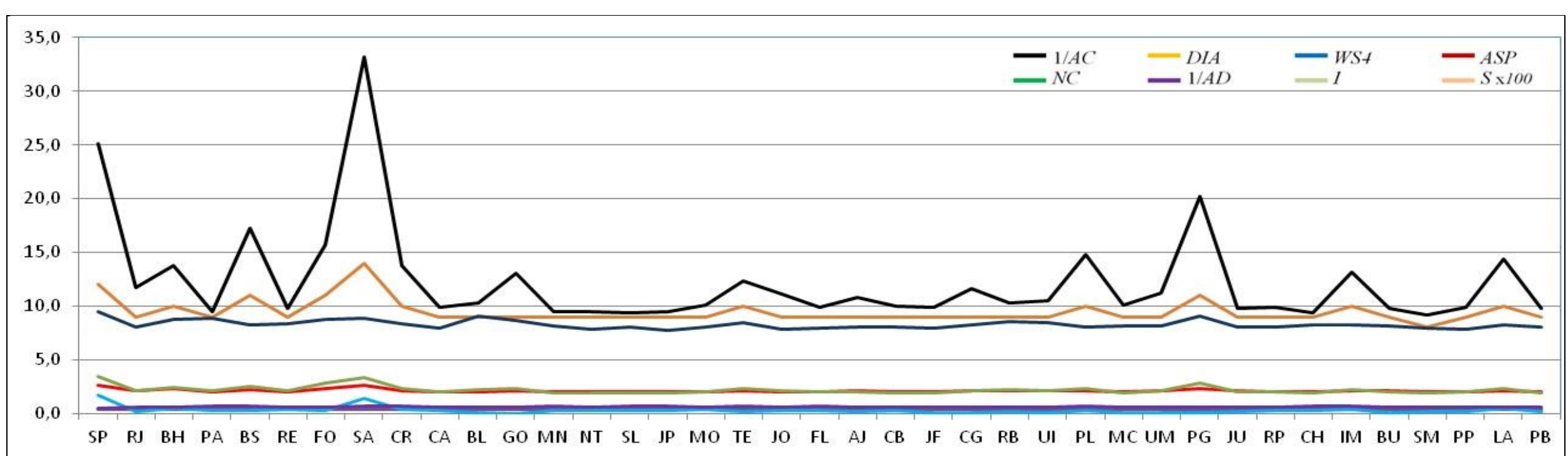

Figura B.1 - medidas após retirada de nós da rede Op_A 


\section{B.2 - Retirada dos nós da rede Op_B}

\begin{tabular}{|c|c|c|c|c|c|c|c|c|c|c|c|c|c|c|c|c|c|c|c|c|c|c|c|c|c|c|c|c|c|c|c|c|c|c|c|c|c|c|c|c|c|}
\hline & & & & & & & & & & & & & & & & & & & & & & & & & & & & & & & & & & & & & & & & & \\
\hline & & & & & & & & & & & & & & & & & & & & & & & & & & & & & & & & & & & & & & & & & \\
\hline & & & & & & & & & & & & & & & & & & & & & & & & & & & & & & & & & & & & & & & & & \\
\hline & & & & & & & & & & & & & & & & & & & & & & & & & & & & & & & & & & & 3 & & & & & & 0 \\
\hline & & 17 & & 17 & & & 12 & & & & & 13 & & & 12 & & 17 & & & 17 & & 18 & & & & & & & & & & & & & & & & & & & 12 \\
\hline & & & & & & & & & & & & & & & & & & & & & & & & & & & & & & & & & & & & & & & & & \\
\hline & & & & & & & & & & & & 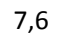 & & 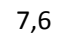 & & & & & & 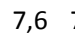 & & & & & & & & & & & & & & & & & & & & & \\
\hline & & & & & & & & & & & & & & & & & & & & & & & & & & & & & & & & & & & & & & & & & \\
\hline & & & & & & & & & & & & & & & & & & & & & & & & & & & & & 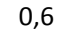 & 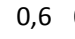 & & 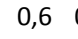 & . & & ,6 & 0,7 & 0,6 & 6 & & 0,6 & , \\
\hline
\end{tabular}

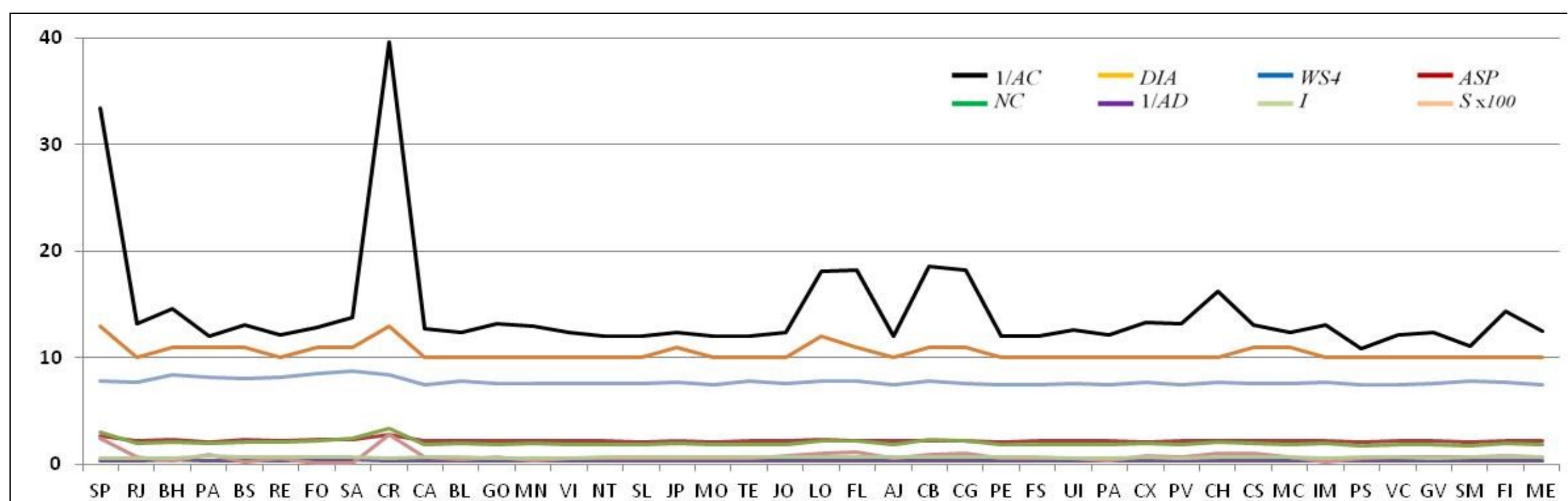

Figura B.2 - medidas após retirada de nós da rede Op_B 


\section{B.3 - Retirada dos nós da rede Op_C}

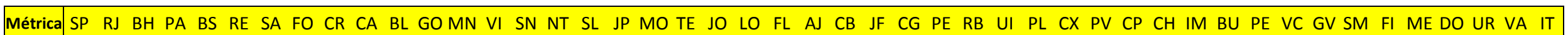

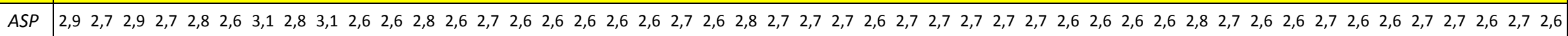

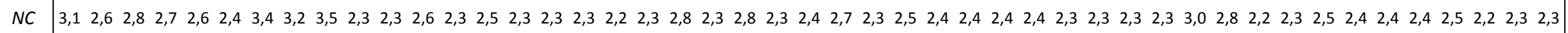
$\begin{array}{lllllllllllllllllllllllllllllllllllllllllllllllllllllllllll}1 / A D & 0,4 & 0,4 & 0,4 & 0,4 & 0,4 & 0,4 & 0,4 & 0,4 & 0,4 & 0,4 & 0,4 & 0,4 & 0,4 & 0,4 & 0,4 & 0,4 & 0,4 & 0,4 & 0,4 & 0,4 & 0,4 & 0,4 & 0,4 & 0,4 & 0,4 & 0,4 & 0,4 & 0,4 & 0,4 & 0,4 & 0,4 & 0,4 & 0,4 & 0,4 & 0,4 & 0,4 & 0,4 & 0,4 & 0,4 & 0,4 & 0,4 & 0,4 & 0,4 & 0,4 & 0,4 & 0,4 & 0,4\end{array}$ $\begin{array}{llllllllllllllllllllllllllllllllllllllllllllllllllllll}1 / A C & 29 & 20 & 20 & 18 & 18 & 15 & 35 & 24 & 38 & 16 & 16 & 19 & 16 & 20 & 16 & 15 & 16 & 15 & 16 & 20 & 16 & 23 & 17 & 17 & 20 & 16 & 19 & 17 & 17 & 17 & 17 & 15 & 17 & 15 & 16 & 25 & 23 & 15 & 17 & 19 & 15 & 17 & 18 & 19 & 15 & 17 & 16\end{array}$ $\begin{array}{lllllllllllllllllllllllllllllllllllllllllllllllllllllllllll}D I A & 15 & 14 & 13 & 15 & 13 & 13 & 18 & 14 & 17 & 13 & 13 & 13 & 13 & 14 & 13 & 13 & 13 & 12 & 13 & 13 & 13 & 14 & 14 & 14 & 13 & 13 & 13 & 14 & 13 & 13 & 13 & 13 & 13 & 13 & 13 & 13 & 13 & 13 & 13 & 13 & 13 & 13 & 14 & 13 & 12 & 13 & 13\end{array}$ WS $\begin{array}{llllllllllllllllllllllllllllllllllllllllllllllllllllllllllll}12 & 11 & 12 & 12 & 11 & 11 & 11 & 12 & 12 & 11 & 11 & 11 & 11 & 11 & 11 & 11 & 11 & 11 & 11 & 11 & 11 & 11 & 11 & 11 & 11 & 11 & 11 & 11 & 11 & 11 & 11 & 11 & 11 & 11 & 11 & 12 & 11 & 11 & 11 & 11 & 11 & 11 & 11 & 11 & 11 & 11 & 11\end{array}$

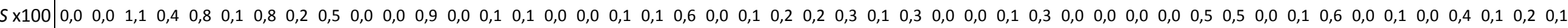

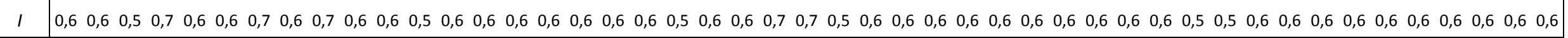

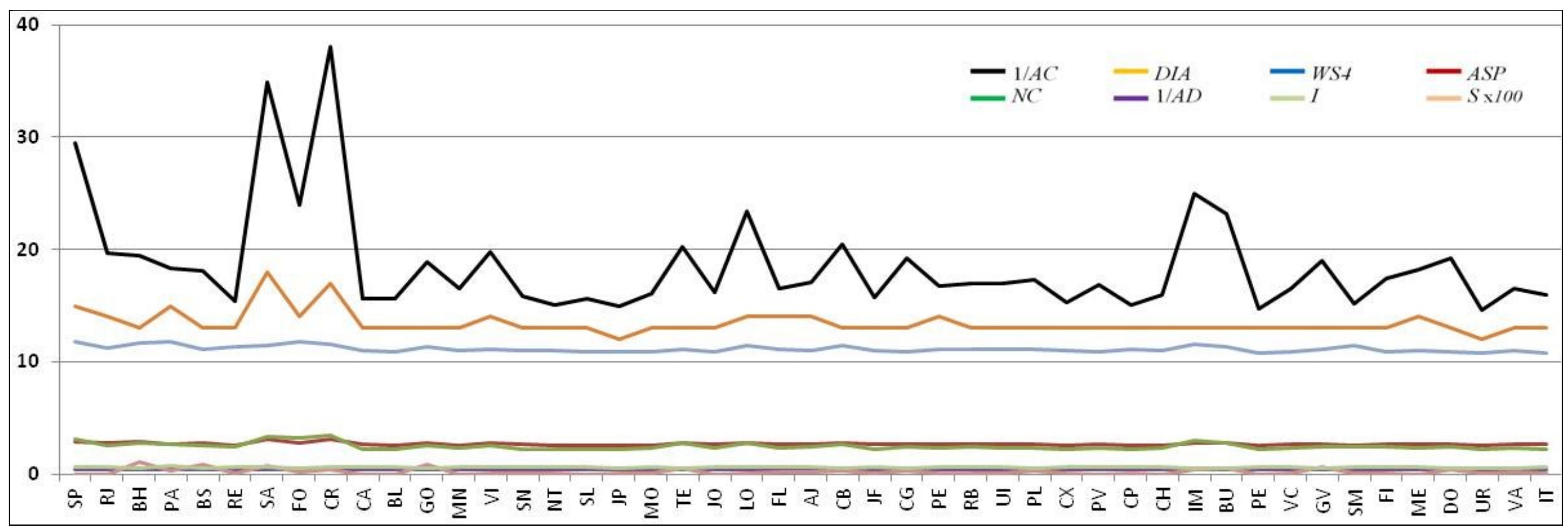

Figura B.3 - medidas após retirada de nós da rede Op_C 


\section{B.4 - Retirada dos nós da rede Op_D}

\begin{tabular}{|c|c|c|c|c|c|c|c|c|c|c|c|c|c|c|c|c|c|c|c|c|c|c|c|c|c|c|c|c|c|c|c|c|c|c|c|c|}
\hline Métrica & $\mathrm{SP}$ & RJ & $\mathrm{BH}$ & PA & BS & RE & FO & SA & $\mathrm{CR}$ & CA & $\mathrm{BL}$ & GO & $\mathrm{MN}$ & $\mathrm{VI}$ & NT & SL & $\mathrm{JP}$ & MO & TE & LO & $\mathrm{FL}$ & AJ & $\mathrm{CB}$ & CG & $\mathrm{RB}$ & UI & PL & PV & IM & $\mathrm{BU}$ & PS & SM & $\mathrm{FI}$ & PF & UR & IT \\
\hline$A S F$ & 3,1 & 2,9 & 2,6 & 2,6 & 2 , & 2,5 & 2,7 & 3,5 & 2,7 & 2,5 & 2,5 & 2,0 & 2,5 & 2,9 & 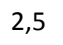 & 2,5 & 5 & 2,5 & 2,5 & 2,6 & 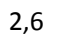 & 2,0 & 2,6 & $2, J$ & 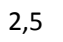 & 2,7 & 2,5 & 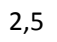 & 2,0 & 2,5 & 2,4 & 2,4 & 2,5 & 5 & 2,4 & 2,8 \\
\hline$N C$ & 3 , & 3,5 & 2,7 & 3,0 & 3,0 & 2,6 & 3,2 & 4,7 & 3,2 & 2,8 & 2,8 & 2,8 & 2,7 & 3,4 & 2,8 & 2,7 & 2,6 & 2,7 & 2,7 & 3,2 & 2,9 & 2,9 & 2,9 & 2,5 & 2,7 & 3,5 & 2,7 & 8 & 3,1 & 2,9 &, 5 & 2,5 & 2,8 & 8 & 2,5 & 3,8 \\
\hline $1 / A D$ & 0 , & 0,4 & 0,4 & 0,4 & 0, & 0,4 & 0,4 & 0,4 & 0,4 & 0,4 & 0,4 & 0,4 & 0,4 & 0,4 & 0,4 & 0,4 & 0,4 & 0,4 & 0,4 & 0,4 & 0,4 & 0,4 & 0,4 & 0,4 & 0,4 & 0,4 & 0,4 & 4 & 0,4 & 0,4 & ,4 &, 4 & 4 & 0.4 & 4 & 0,4 \\
\hline $1 / A C$ & 39 & 32 & 19 & 20 & 19 & 17 & 19 & 44 & 26 & 20 & 17 & 19 & 18 & 31 & 17 & 17 & 17 & 18 & 17 & 24 & 21 & 19 & 20 & 17 & 19 & 31 & 18 & 19 & 18 & 22 & 16 & 16 & 20 & 8 & 16 & 32 \\
\hline$D I A$ & 15 & 14 & 12 & 14 & 13 & 12 & 13 & 20 & 13 & 12 & 11 & 12 & 12 & 14 & 12 & 12 & 12 & 12 & 12 & 12 & 13 & 13 & 14 & 12 & 12 & 13 & 12 & 13 & 12 & 12 & 12 & 12 & 2 & 12 & 11 & 15 \\
\hline WS4 & 9,6 & 8,9 & 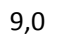 & , & 9,1 & 8,8 & 9,4 & , & W & 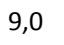 & 9,0 & 8,8 & 8,8 & 8,8 & 8,9 & שונה & 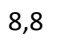 & 8,8 & 8,8 & 9,1 & 0 & 9,0 & D, & 3,8 & $0, r$ &, 0 & (כ, & 8 & 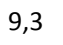 & 3 & 8,9 & 9,0 & 8 & 9 & 6 & 9,2 \\
\hline$S \times 100$ & 0,1 & 2,1 & 1,6 & 0,4 & 1,7 & 0,7 & 1,8 & 0,9 & 0,3 & 1,2 & 1,9 & 1,8 & 1,1 & 1,9 & 1,1 & 1,2 & 0,8 & 0,9 & 1,2 & 0,7 & 0,1 & 1,2 & 0,5 & 0,9 & 1,2 & 2,1 & 1,3 & 0,7 & 1,9 & 1,2 & 0,7 & 0,7 & 0,7 & 0,7 & 1,3 & 1,0 \\
\hline I & 0,5 & 0 , & 0,5 & 6 & 0,5 & 6 & 0,5 & 0,6 & 0,5 & 5 & 0,4 & 0,5 & 5 & 5 & 0,5 & 0,5 & 6 & 0,5 & 0,5 & 0,5 & 0,5 & 0,6 & 0,6 & 0,5 & 0,5 & 0,5 & 0,5 & 0,6 & 0,5 & 0,5 & 0,6 & 0,6 & 0,5 & 0,6 & 0,5 & 0,6 \\
\hline
\end{tabular}

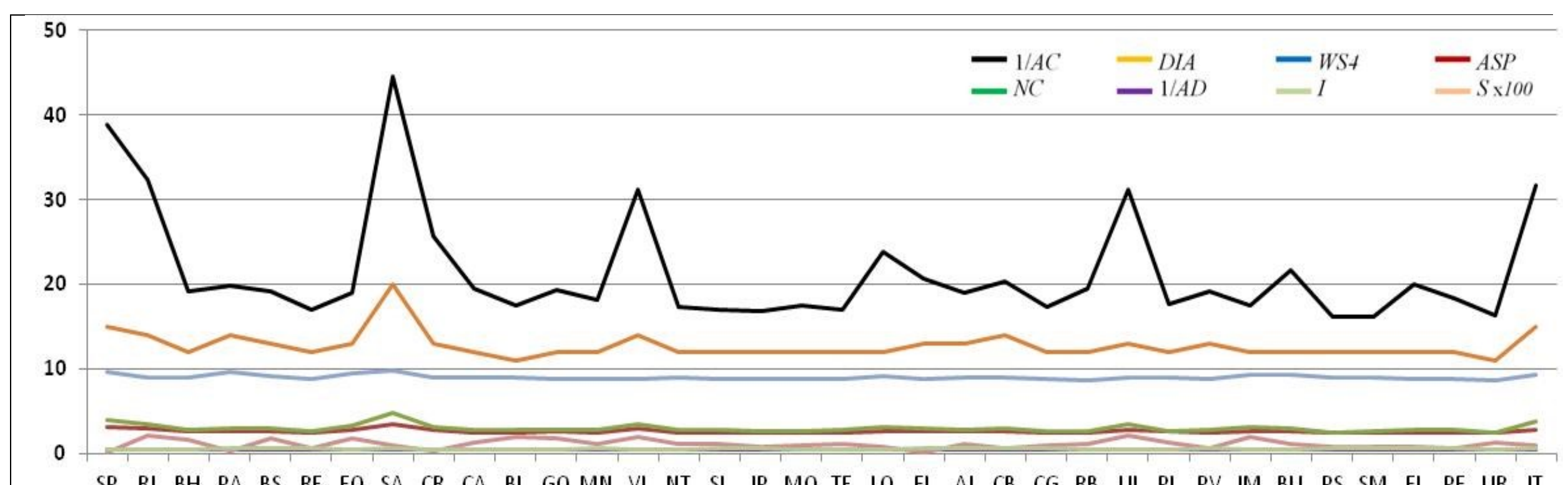

SP RJ BH PA BS RE FO $S A$ CR CA BL GO MN VI NT SL JP MO TE LO

Figura B.4 - medidas após retirada de nós da rede Op_D 


\section{B.5 - Retirada dos nós da rede Op_E}

\begin{tabular}{|c|c|c|c|c|c|c|c|c|c|c|c|c|c|c|c|c|c|c|c|c|c|c|c|c|c|c|c|c|c|c|c|c|c|c|c|c|c|c|c|c|}
\hline Métrica & SP & RJ & $\mathrm{BH}$ & PA & BS & $\mathrm{RE}$ & FO & SA & $\mathrm{CR}$ & CA & $B L$ & SJ & $\mathrm{GO}$ & MN & VI & SN & NT & SL & $\mathrm{JP}$ & $\mathrm{MO}$ & $\mathrm{JO}$ & LO & $\mathrm{FL}$ & AJ & so & $\mathrm{CB}$ & CG & $\mathrm{RB}$ & $\mathrm{PL}$ & $C X$ & $\mathrm{RP}$ & PV & $\mathrm{CP}$ & $\mathrm{CH}$ & BU & GV & SM & $\mathrm{FI}$ & PP & DO \\
\hline$A S F$ & 2, & 2,3 & 2 & 2,3 & 2,9 & 2 & 2,4 & 2,5 & 2,7 & 2,3 & 2,6 & 2,3 & 2,4 & 2,3 & ,3 & 2,3 & 2,3 & 2 & ,3 & ,4 & 2,3 & 2,3 & ,3 & 2,4 & 2,3 & 2,5 & 2,4 & 2,4 & 2,5 & 2,3 & 2,3 & 2,3 & 2,3 & 2,3 & 2,3 & 4 & 2,3 & 2,3 & 2,3 & 2,3 \\
\hline$\Lambda$ & 2,9 & 2,3 & 25 & 27 & 30 & 2,6 & 28 & 29 & 3 & 2,1 & 32 & 2,2 & 2 & 23 & 2,2 & 2,1 & 2 & 2,8 & 21 & 2,6 & 2 & 22 & 27 & 7 & 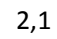 & 2,7 & 2,4 & 4 & 23 & 2 & 2,1 & 2,3 & 21 & ,5 & 4 & 22 & 21 & 21 & 21 & 2,4 \\
\hline $1 / A D$ & 0, & 0,4 & 0 & 0,4 & 0,4 & 0,4 & 0 & 0,4 & 0,4 & 0,4 & 20 & 0,4 & 0,4 & 0,4 & 0,4 & 0,4 & 0,4 & 0,4 & 0,4 & 0,4 & 0,4 & 0,4 & 0,4 & 0,4 & 0,4 & 0,4 & 0,4 & 0,4 & 0,4 & 0,4 & 0,4 & 0,4 & 0,4 & 0,4 & 0,4 & 0,4 & 0,4 & 0,4 & 0, & 0,4 \\
\hline $1 / A C$ & 18 & 14 & 15 & 11 & 20 & 17 & 21 & 25 & 27 & 12 & 28 & 12 & 12 & 14 & 13 & 12 & 11 & 24 & 10 & 19 & 12 & 12 & 12 & 22 & 1 & 18 & 15 & 14 & 15 & 11 & 12 & 4 & 1 & 14 & 12 & 13 & 1 & 11 & 12 & 14 \\
\hline$D$ & 1 & 1 & 1 & 11 & 1 & 11 & 12 & 13 & 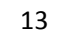 & 11 & 13 & 11 & 1 & 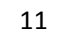 & 11 & 11 & 11 & -1 & 10 & 11 & 11 & 11 & 11 & 12 & 11 & 13 & 12 & 11 & 11 & \pm & 11 & 11 & 1 & + & + & 4 & 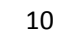 & 11 & 11 & $I+$ \\
\hline WS4 & 10,3 & 9,4 & 9,8 & 9,3 & 10,0 & 9,8 & 9,7 & 9,5 & 10,3 & 9,4 & 9,6 & 9,4 & 9,4 & 9,3 & 9,3 & 9,3 & 9,3 & 9,5 & 9,4 & 9,4 & 9,5 & 9,5 & 9,5 & 9,3 & 9,4 & 9,7 & 9,3 & 9,7 & 9,4 & 9,4 & 9,0 & 9,4 & 9,5 & 9,5 & 9,7 & 4 & 9,2 & 9,2 & 9,0 & 9,4 \\
\hline$S \times 10$ & 0,2 & si & 1,4 & 0,0 & 2 & 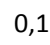 & 0,6 & 0,6 & 0, & 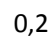 & 0,7 & 0,1 & 0, & 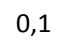 & 0,0 & 0 , & 0 & C & 0,0 & 0,2 & 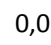 & 0,2 & 0,1 & 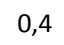 & 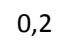 & 0,1 & 0,1 & 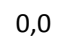 & 00 & 01 & 0,1 & 0,1 & 0,1 & 0,3 &, 2 & 0,2 & 01 & 01 & 01 & 0,2 \\
\hline 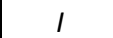 & 0,6 & 0,6 & 0,4 & 0,6 & 0,5 & 0,6 & 0,0 & 0,0 & 0,5 & 0,0 & 0 & 0,6 & 0,5 & 0,0 & 0,6 & 0,6 & 0,6 & 0,6 & 0 & 0,6 & 0,6 & s & , & 0,6 & 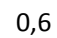 & 0,0 & 0,0 & 0,0 & & 0,0 & 0,0 & 0,0 & 0,0 & 0,0 & 0,6 & 0,6 & , J & & 0,0 & 0,0 \\
\hline
\end{tabular}

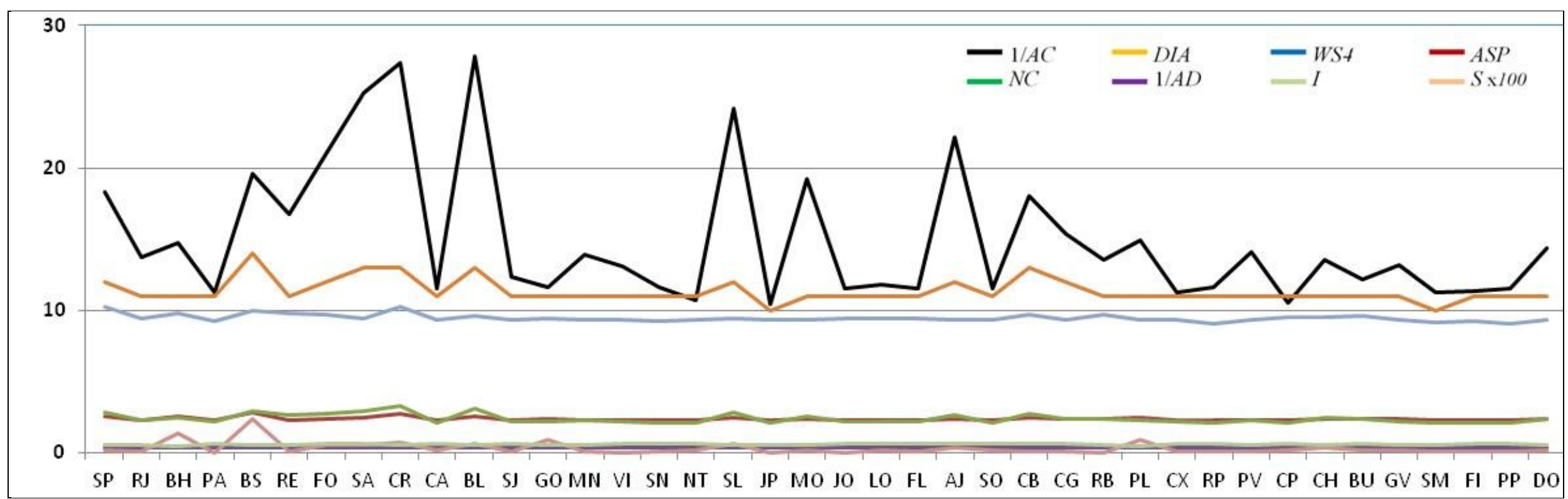

Figura B.5 - medidas após retirada de nós da rede Op_E 


\section{B.6 - Retirada dos nós da rede Cost266}

\begin{tabular}{|c|c|c|c|c|c|c|c|c|c|c|c|c|c|c|c|c|c|c|c|c|c|c|c|c|c|c|c|c|c|c|c|c|c|c|c|c|c|}
\hline Métri & LD & L & MD & RM & AT & $\mathbf{R}$ & B & BP & WS & VN & BN & $\mathrm{ZC}$ & SF & MC & ML & PG & BG & LN & $S$ & H & MS & SH & $\mathbf{R}$ & $\mathbf{R}$ & $A D$ & V & K & 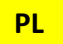 & OS & HS & GG & DD & CG & DB & LB & BD & SB \\
\hline$A S F$ & & 3 & & 2,1 & 1,9 & 1,9 & 2,0 & 1,9 & 1, & 1, & 1, & 1, & 1,8 & 2,0 & 1 & 1,9 & 1,9 & 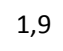 & 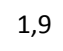 & 1,9 & 2,0 & 8 & 9 & 9 & 2,0 & 9 & 9, & 9 & L,8 & 1,9 & 1,9 & 1,9 & 1,9 & 1,9 & 1,9 & 1,9 & 19 \\
\hline$N C$ & 18 & 24 & 14 & 18 & 15 & 16 & 16 & 1,6 & 16 & 1,5 & 1,5 & 1,5 & 1,4 & 1,6 & 1,5 & 1,5 & 1,5 & 1,5 & 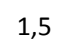 & 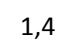 & 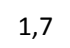 & r & 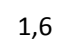 & 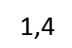 & t & 1,4 & 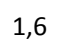 & 1,4 & 1,4 & 1,5 & 5 & 1,4 & 1,6 & 14 & 1,5 & 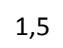 & 1,5 \\
\hline $1 / A D$ & 0 & 0,3 & 0,3 & 0,3 & 0,3 & 0,3 & 0,3 & 0,3 & 0,3 & 0,3 & 0,3 & 0,3 & 0,3 & 0,3 & 0,3 & 0,3 & 0,3 & 3 & P & 0,3 & 0,3 & 3 & 3 & ,3 & 0,3 & 0,3 & 0,3 & 0,3 & 0,3 & 0,3 & 0,3 & 3 & 3 & 3 & ,3 & 0,3 & 0,3 \\
\hline $1 / A$ & 7 & 20 & 6 & 10 & 7 & 7 & 9 & 7 & 7 & 7 & 7 & 7 & 6 & 8 & 7 & 6 & 7 & 6 & 6 & 6 & $c$ & 6 & 7 & 6 & s & & & 7 & 6 & 6 & 6 & 7 & 8 & 6 & 6 & 6 & 7 \\
\hline$D$ & 9 & 1 & 8 & + & 8 & 8 & 9 & 8 & 8 & 8 & 8 & 8 & 7 & 8 & 8 & 8 & 8 & 8 & 8 & 8 & 10 & & 0 & 8 & 9 & & & & & & & 0 & 0 & 0 & 8 & 0 & 8 \\
\hline WS4 & 8 & 8,4 & 7,5 & 8,0 & 7,5 & 8,1 & 7,8 & 7,8 & 8,0 & 7,7 & 7,5 & 7,5 & 7,5 & 7,8 & 7,8 & 7,7 & 7,7 & 7,6 & 7,7 & 7,4 & 8,0 & 7,4 & 8,0 & 7,5 & 8,0 & 7,4 & 7,8 & 7,5 & 7,4 & 7,6 & 7,3 & 7,5 & 7,7 & 7,4 & 7,7 & 7,7 & 7,7 \\
\hline$x 10$ & 0,0 & 1,4 & 2 & 0,1 & 1 & 20 & $0 ?$ & $0=$ & 0. & 0 & 0, & 0 , & 0 , & 0 & 0 & 0 & 0 & 0,2 & 0,3 & 0,2 & 0,1 & 0,1 & 0,5 & 0,2 & 0,2 & 0,2 & 0.5 & ? & 0,1 & 0,1 & 2 & 3 & 0 & 2 & 1 & 2 & 0,2 \\
\hline & & 7 & 0 & 6 & 0 & 6 & כ & 0 & כ) & , &, 0 & 0,6 & 0,5 & 0,5 & 0,5 & 0,0 & 0,0 & 0,0 & 0 & , & , & 0,6 & 0,5 & 0,6 & 0,6 & 0,6 & 0,6 & 0,6 & 0,6 & 0,6 & 0,0 & 0,0 & 0,5 & 0,6 & 0,6 & 0,6 & 0,6 \\
\hline
\end{tabular}

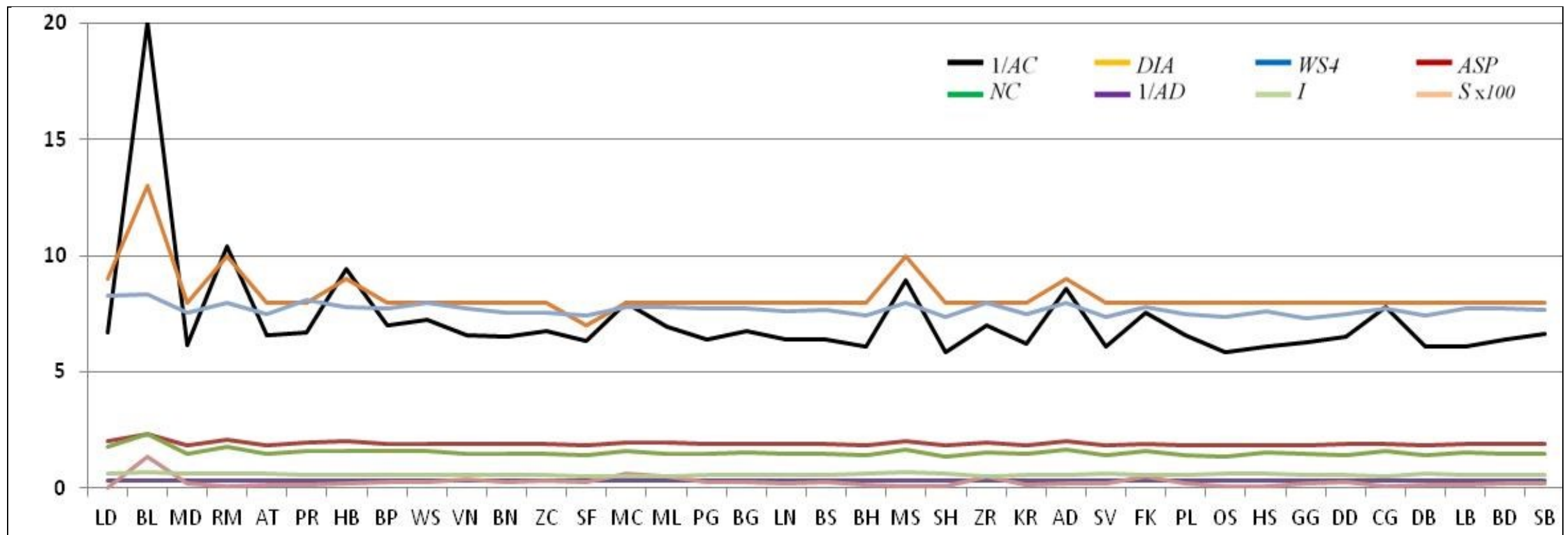

Figura B.6 - medidas após retirada de nós da rede Cost266 


\section{B.7 - Retirada dos nós da rede Janos US-CA}

\begin{tabular}{|c|c|c|c|c|c|c|c|c|c|c|c|c|c|c|c|c|c|c|c|c|c|c|c|c|c|c|c|c|c|c|c|c|c|c|c|c|c|c|c|}
\hline Métric & $Y$ & LA & CC & $\mathrm{DL}$ & PD & IS & $\mathrm{T}$ & & n & AT & B & SF & $\mathrm{T}$ & $\mathrm{N}$ & ST & IR & MP & SD & SL & TP & $\mathrm{JV}$ & PB & IC & $\mathrm{DL}$ & SM & $\mathrm{CN}$ & $\mathrm{CL}$ & C & LV & $P$ & NV & $\mathrm{MH}$ & $\mathrm{OH}$ & CG & 10 & SC & EP & VP & $C R$ \\
\hline$A S F$ & 2 & 2,1 & 2,3 & 2,2 & 2,1 & 2,1 & 2,1 & 2.2 & 2,1 & 2,1 & 2 & 2 & 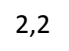 & 2,1 & 2,1 & 1 & 2,2 & 1 & 2 & 2,1 & 2,2 & 2,1 & 2,1 & , & 1 & 1 & 1 & ,2 & 1 & ,2 &, 2 & 2 & 1 & ,2 & 2 & ,3 & ,2 & 1 & 2,2 \\
\hline$\Lambda$ & 1,8 & 16 & 1,9 & 18 & 16 & 1,7 & 1,7 & 1,8 & 1,5 & 1,6 & 1,5 & 1 & 1,8 & 1,0 & 1,6 & 1,5 & 1,8 & 1,5 & 1,7 & 1 & 17 & 15 & 1,6 & 1,7 & 6 & ,6 & 1,7 & 7 & 1,6 & I & 1,6 & 1,7 & 1,5 & 8 & 1,8 & 9, & 8 & ,7 & 1,8 \\
\hline $1 / A D$ & 0,3 & 0,3 & 0,3 & 0,3 & 0,3 & 0,3 & 0,3 & 0,3 & 0,3 & 0,3 & 0,3 & 0,3 & 0,3 & 0,3 & 0,3 & 0,3 & 0,3 & 0,3 & 0,3 & 0,3 & 0,3 & 0,3 & 0,3 & 0,3 & 0,3 & 0,3 & 0,3 & 0,3 & 0,3 & 0,3 & 0,3 & 0,3 & 0,3 & 0,3 & 0,3 & 0,3 & 0,3 & 0,3 & 0,3 \\
\hline $1 / A$ & 9 & 9 & 14 & 10 & 9 & 1 & 9 & 11 & 9 & 9 & 9 & 9 & 1 & 1 & 9 & 9 & 13 & 9 & 10 & 9 & 12 & 9 & 9 & 9 & 9 & 10 & 10 & 10 & 9 & 10 & 9 & 10 & 9 & 11 & 11 & 2 & 12 & 11 & 12 \\
\hline$D$ & 1 & 10 & 11 & 10 & 10 & 10 & 11 & 10 & 10 & 10 & 9 & 10 & 11 & 11 & 10 & 10 & 10 & 10 & 10 & 10 & 10 & 10 & 10 & 10 & 10 & 10 & 10 & 10 & 11 & 10 & 10 & 10 & 10 & 11 & 10 & 1 & 11 & 10 & 10 \\
\hline n & 8 & 7,5 & 7,9 & 7. & 76 & 77 & 7,7 & 7,6 & 7,4 & 7 & 7,3 & 7,6 & 7,8 & 76 & 4 & 3 & 7 & 7,4 & 7,8 & 7,5 & 7,7 & 7,5 & 7,4 & 7,9 & 7,6 & 7,5 & 7,9 & 7,6 & 7,9 & 7,8 & 7,7 & 7,8 & 7,5 & , & 7,7 & 4 & 7,8 & ,5 & 3,1 \\
\hline$S \times 10$ & 0,1 & 0,2 & 0,1 & 0,7 & 6,1 & 0,0 & 0,5 & 0,1 & 0,2 & 0,1 & 0,2 & 0,2 & 0,4 & 0,2 & 0,2 & 0,2 & 0,1 & 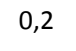 & 0 & 0 & 0,5 & 0,2 & 0,3 & 0,2 & 0,2 & 0,2 & 0,2 & 0,2 & 0,3 & 0,1 & 0 & , 2 & 1 & ,3 & 1 & ,2 & $\perp$ & 0,2 & 0,1 \\
\hline I & 0,6 & 0,6 & 0,5 & 0,5 & 0,6 & 0,6 & 0,7 & 0,5 & 0,6 & 0,6 & 0,5 & 0,6 & 0,6 & 0,7 & 0,6 & 0,6 & 0,5 & 0,6 & 0,5 & 0,6 & 0,5 & 0,6 & 0,6 & 0,6 & 0,6 & 0,6 & 0,6 & 0,6 & 0,7 & 0,6 & 0,6 & 0,5 & 0,6 & 0,6 & 0,5 & 0,6 & 0,6 & 0,5 & 0,5 \\
\hline
\end{tabular}

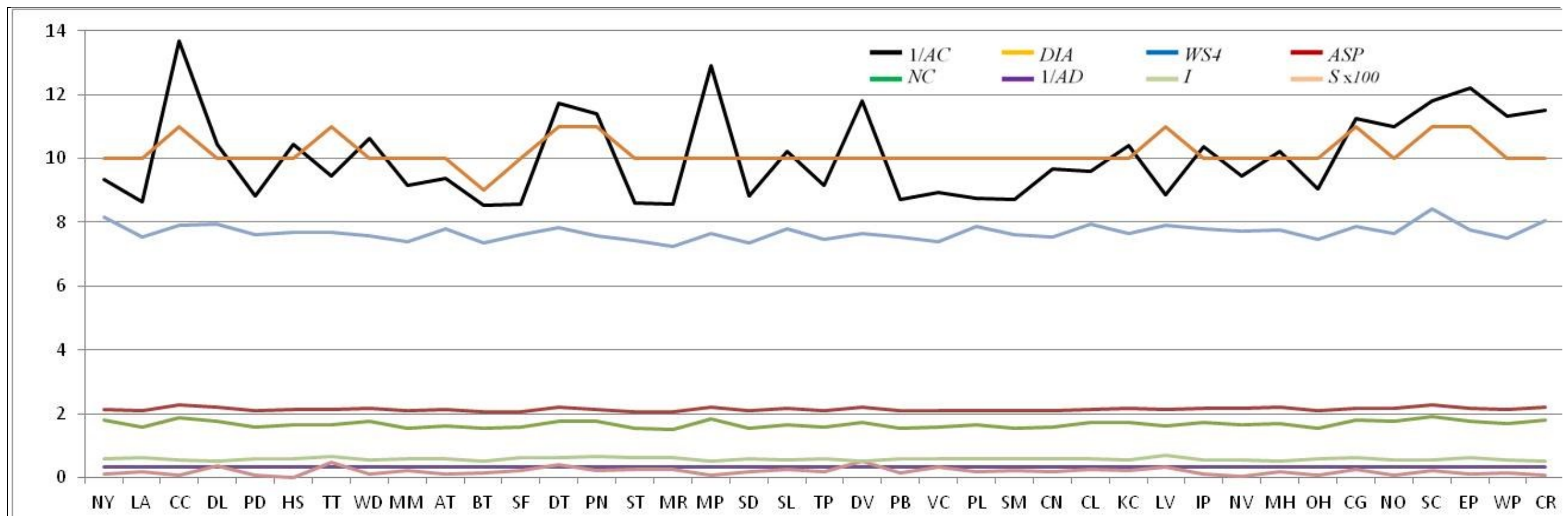

Figura B.7 - medidas após retirada de nós da rede US-CA 


\section{B.8 - Retirada de enlaces da rede Op_A}

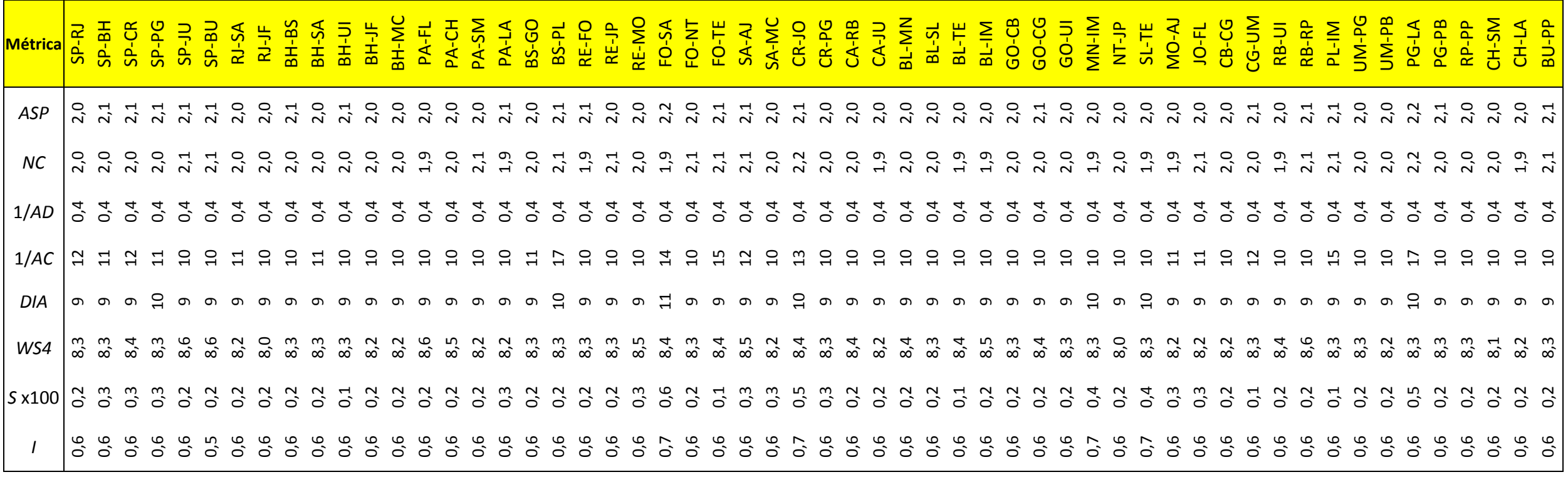

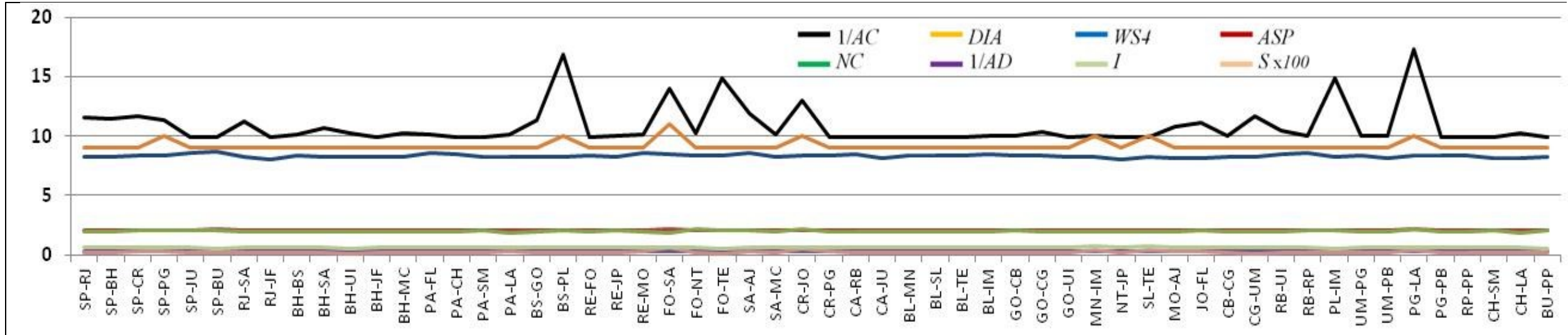

Figura B.8 - medidas após retirada de enlaces da rede Op_A 


\section{B.9 - Retirada de enlaces da rede Op_B}

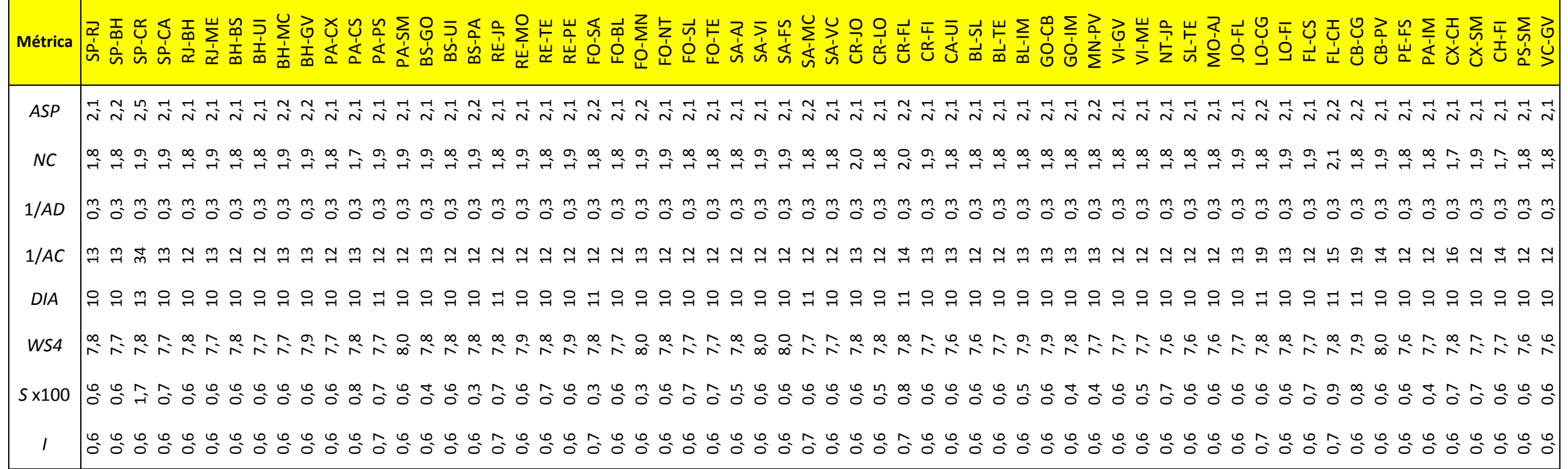

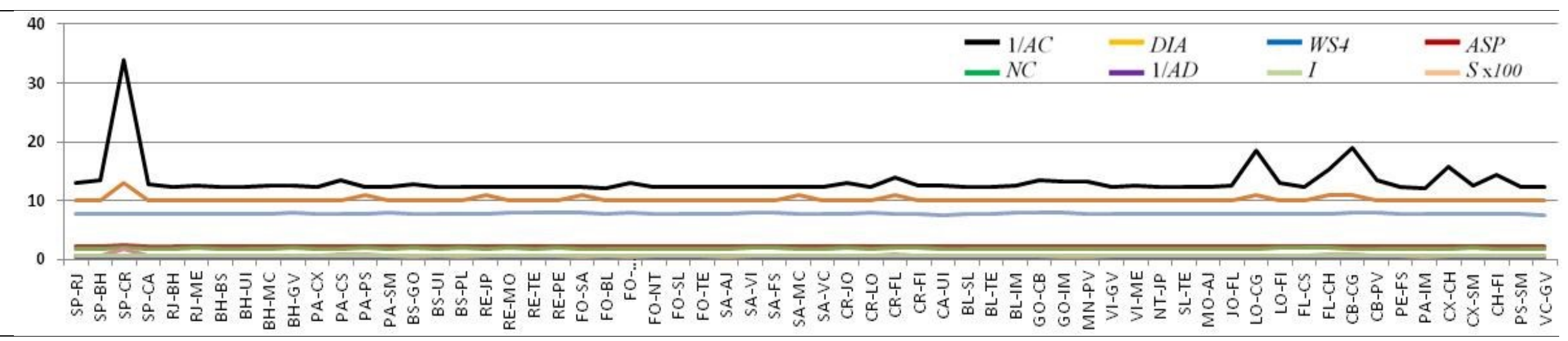

Figura B.9 - medidas após retirada de enlaces da rede Op_B 


\section{B.10 - Retirada de enlaces da rede Op_C}

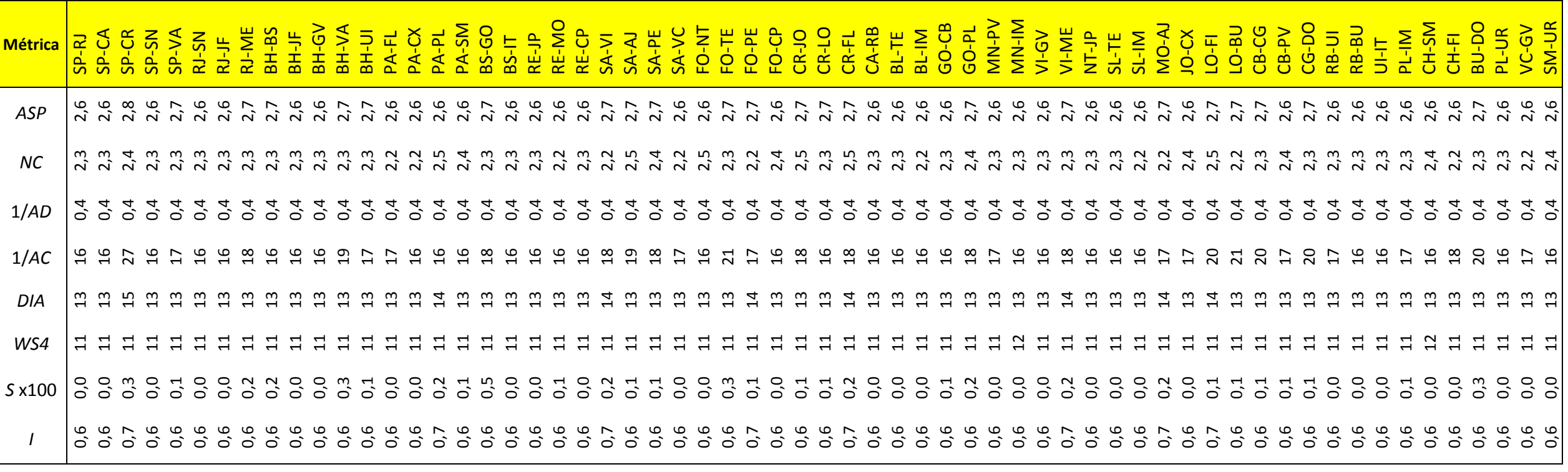

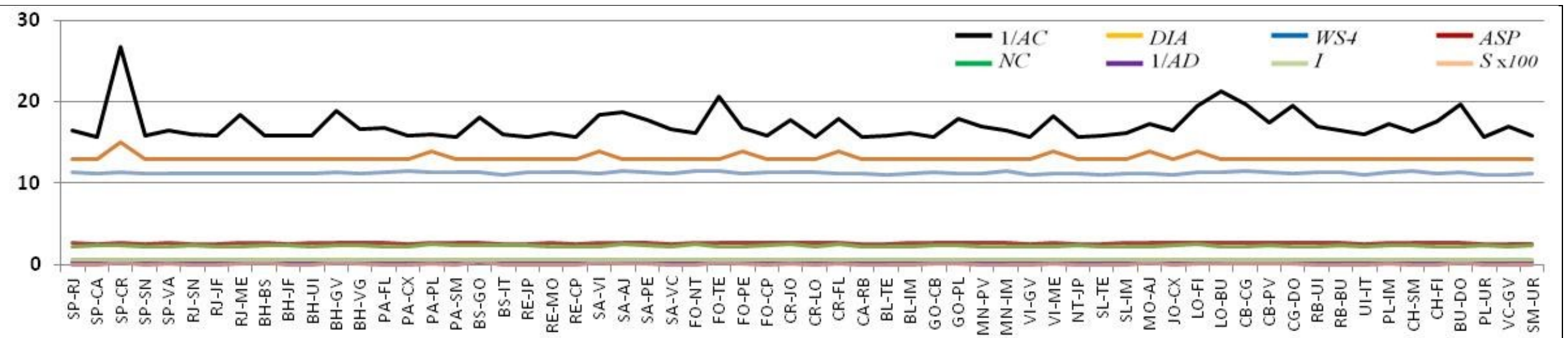

Figura B.10 - medidas após retirada de nós da rede Op_C 


\section{B.11 - Retirada de enlaces da rede Op_D}

\begin{tabular}{|c|c|c|c|c|c|c|c|c|c|c|c|c|c|c|c|c|c|c|c|c|c|c|c|c|c|c|c|c|c|c|c|c|c|c|c|c|c|c|c|c|c|c|c|c|c|c|c|}
\hline étrica & 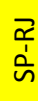 & & $\begin{array}{l}\text { Ù } \\
\vdots \\
\text { ì }\end{array}$ & \begin{tabular}{l} 
\} $\\
{\vdots} \\
{\text { जे }}$ & $\begin{array}{l}\stackrel{\partial}{0} \\
\vdots \\
\text { iे }\end{array}$ & $\begin{array}{l}\frac{I}{0} \\
\frac{1}{\alpha}\end{array}$ & $\underset{\frac{1}{\alpha}}{>}$ & $\begin{array}{l}\overline{1} \\
\frac{1}{1} \\
\infty\end{array}$ & $\frac{\overrightarrow{1}}{\frac{1}{\Delta}}$ & $\frac{\Delta}{\frac{a}{\alpha}}$ & $\frac{\omega}{\frac{\alpha}{\alpha}}$ & $\sum_{\substack{\frac{1}{\alpha} \\
\vdots}}$ & $\begin{array}{l}\overleftarrow{n} \\
\text { ஸे }\end{array}$ & $\begin{array}{l}\text { О } \\
\text { ஸे } \\
\text { ஸे }\end{array}$ & $\begin{array}{l}\overrightarrow{0} \\
\dot{\sim} \\
\ddot{\infty}\end{array}$ & 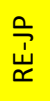 & $\sum_{\substack{\sim \\
\sim}}^{O}$ & $\begin{array}{l}\text { 屯ิ } \\
\text { ồ }\end{array}$ & $\begin{array}{l}\sum_{1} \\
\text { o }\end{array}$ & 혼 & $\underset{\frac{1}{4}}{\frac{1}{5}}$ & 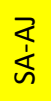 & $\begin{array}{l}\frac{1}{1} \\
\text { c) }\end{array}$ & $\begin{array}{l}\frac{\overrightarrow{1}}{\alpha} \\
\stackrel{\frac{v}{u}}{0}\end{array}$ & $\begin{array}{l}\frac{\infty}{\not c} \\
\frac{1}{j}\end{array}$ & $\begin{array}{l}D \\
0 \\
\dot{\delta}\end{array}$ & 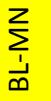 & $\underset{⿱ 亠 凶}{\grave{\Delta}}$ & 卣 & & $\frac{a}{\vec{S}}$ & 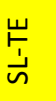 & $\sum_{\substack{\Delta \\
\sim}}$ & 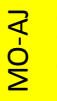 & 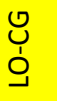 & $\begin{array}{l}\text { o. } \\
\text { ò }\end{array}$ & $\begin{array}{l}\bar{\bigsqcup} \\
\text { @़ }\end{array}$ & ồ & 톻 & $\begin{array}{l}\text { o } \\
\text { 今े } \\
\text { U }\end{array}$ & $\begin{array}{l}\overline{1} \\
\stackrel{1}{\alpha}\end{array}$ & $\stackrel{T}{=}$ & $\frac{\pi}{\frac{1}{2}}$ & 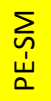 & $\frac{\stackrel{9}{\rho}}{\sum_{n}^{1}}$ & $\frac{\frac{u}{a}}{\frac{1}{4}}$ & 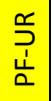 \\
\hline$A S P$ & & & $i$ & $\stackrel{n n}{\sim}$ & $\stackrel{n n}{\sim}$ & $\stackrel{n}{\sim}$ & i & $\stackrel{n}{\sim}$ & i & $N$ & $\stackrel{n}{\sim}$ & $\stackrel{\stackrel{n}{N}}{\sim}$ & 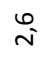 & $\stackrel{\stackrel{n}{N}}{\sim}$ & $\stackrel{0}{i}$ & $\stackrel{\sim}{\sim}$ & $\stackrel{n}{\sim}$ & 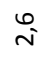 & $\stackrel{n}{\sim}$ & $\stackrel{\sim}{\sim}$ & $\hat{i}$ & 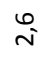 & $\stackrel{\sim}{\sim}$ & 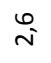 & 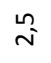 & $\stackrel{n}{\sim}$ & $\stackrel{n}{\sim}$ & $\stackrel{n}{\sim}$ & $\stackrel{\bullet}{\sim}$ & $\stackrel{n}{\sim}$ & $\stackrel{n}{\sim}$ & $\stackrel{n}{\sim}$ & $\stackrel{n}{\sim}$ & $\stackrel{0}{i}$ & $\stackrel{n}{\sim}$ & $\stackrel{n}{\sim}$ & 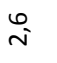 & $\stackrel{0}{i}$ & $\stackrel{0}{i}$ & $\stackrel{n}{\sim}$ & $\stackrel{n}{\sim}$ & 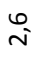 & $\tilde{v}$ & 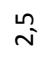 & 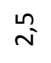 & $\stackrel{n}{\sim}$ & $N$ \\
\hline$N C$ & & $\stackrel{n}{\sim}$ & $\stackrel{\alpha}{n}$ & $\begin{array}{l}0 \\
\sim\end{array}$ & $\hat{i}$ & $\stackrel{b}{\sim}$ & & $N$ & $\sim$ & N & N & $\hat{i}$ & $\stackrel{0}{i}$ & $\stackrel{n}{N}$ & $\stackrel{\infty}{\sim}$ & $\stackrel{0}{i}$ & $\stackrel{n}{N}$ & $\stackrel{n}{N}$ & $\stackrel{\text { à }}{\sim}$ & $\hat{i}$ & $\stackrel{+}{\sim}$ & $\stackrel{\text { ș }}{\sim}$ & $\stackrel{0}{i}$ & $\stackrel{\leftrightarrow}{\sim}$ & $\stackrel{n}{N}$ & $\hat{i}$ & $\stackrel{0}{i}$ & $\stackrel{0}{\sim}$ & $\stackrel{0}{i}$ & $\stackrel{n}{\sim}$ & $\stackrel{\infty}{\sim}$ & $\stackrel{0}{i}$ & $\stackrel{0}{i}$ & $\stackrel{n}{\sim}$ & $\stackrel{0}{i}$ & $\stackrel{n}{\sim}$ & $\stackrel{a}{i}$ & $\stackrel{\infty}{\sim}$ & $\stackrel{n}{\sim}$ & $\stackrel{n}{\sim}$ & $\stackrel{n}{\sim}$ & $\sim$ & $\hat{\sim}$ & $\stackrel{0}{i}$ & $\stackrel{0}{i}$ & $\stackrel{\sigma}{\sim}$ & 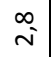 \\
\hline $1 / A D$ & c & & $\stackrel{+}{0}$ & $\stackrel{+}{0}$ & $\stackrel{\square}{0}$ & $\stackrel{\nabla}{0}$ & $\stackrel{\square}{\circ}$ & ఫ্ & $\sigma^{\circ}$ & $\stackrel{\Delta}{\circ}$ & ¿্ & $\stackrel{+}{0}$ & $\stackrel{\nabla}{0}$ & $\stackrel{\nabla}{0}$ & $\stackrel{+}{0}$ & $\stackrel{+}{0}$ & $\stackrel{\nabla}{0}$ & $\stackrel{\nabla}{\circ}$ & $\stackrel{+}{0}$ & $\stackrel{+}{0}$ & $\stackrel{\nabla}{0}$ & $\stackrel{\nabla}{0}$ & $\stackrel{\Delta}{\circ}$ & $\stackrel{+}{\circ}$ & $\stackrel{\Delta}{\circ}$ & ¿ & ণ্ & ণ & t & $\stackrel{\Delta}{\circ}$ & ণ্ & t্ & t) & t) & ণ & t & ¿ & t্ & ণ & t) & ‡ & $\stackrel{+}{\circ}$ & $\stackrel{\Delta}{\circ}$ & $\stackrel{\Delta}{\circ}$ & t & ث্ & s \\
\hline $1 / A C$ & $\pi$ & 국 & $\approx$ & ને & $\stackrel{\infty}{\rightarrow}$ & $\stackrel{\infty}{\rightarrow}$ & $\tilde{m}$ & ન & $\vec{N}$ & ને & 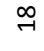 & $\stackrel{\infty}{\rightarrow}$ & ने & ન્ & $\stackrel{\infty}{\rightarrow}$ & $\hat{\neg}$ & $\stackrel{\infty}{\rightarrow}$ & $\stackrel{N}{ }$ & ન્ & $\stackrel{\infty}{\rightarrow}$ & $\tilde{m}$ & $\vec{N}$ & $\hat{\neg}$ & $\stackrel{\sim}{\sim}$ & 가 & $\stackrel{\infty}{\rightarrow}$ & $\stackrel{\infty}{\sim}$ & $\stackrel{\infty}{\rightarrow}$ & $\stackrel{N}{ }$ & 구 & $\alpha$ & $n$ & 0 & 구 & $\stackrel{\infty}{\sim}$ & $\stackrel{\infty}{\sim}$ & 3 & ㄱ & $\approx$ & $\stackrel{\infty}{\sim}$ & $\stackrel{N}{ }$ & $\vec{m}$ & $\infty$ & $\hat{ન}$ & $\curvearrowright$ & i & \\
\hline$D I A$ & 7 & $\approx$ & $\stackrel{m}{\rightarrow}$ & テ & 강 & ㄱ & $\mathcal{I}$ & $\underset{-}{-1}$ & $\because$ & 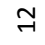 & $\underset{-}{-1}$ & ㄱ & ㄱ & ㄱ & ユ & ㄱ & ㄱ & 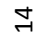 & ユ & ㄱ & 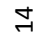 & ㄱ & ㄱ & $\stackrel{m}{\rightarrow}$ & ㄱ & ㄱ & $\approx$ & $\approx$ & フ & $\stackrel{m}{-}$ & $\underset{7}{*}$ & $\approx$ & Ұ & $\ddot{-}$ & $\underset{7}{*}$ & $\approx$ & $\approx$ & $\approx$ & $\stackrel{\sim}{\sim}$ & $\approx$ & ㄱ & 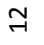 & 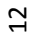 & ㄱ & $y$ & & . \\
\hline WS4 & $\sigma$ & & $\sigma$ & $\sigma$ & $\sigma$ & Oे & $\stackrel{\circ}{\alpha}$ & & & $\sigma$ & $\sigma$ & $\stackrel{-1}{\sigma}$ & $\tilde{\sigma}$ & $\vec{\sigma}$ & $\stackrel{-1}{\sigma}$ & $m_{\sigma}^{m}$ & $m_{\sigma}^{m}$ & $\stackrel{m}{\sigma}$ & $m_{\sigma}^{m}$ & $\tilde{\sigma}$ & $\vec{\sigma}$ & హ̆ & ò & ò & ò & ๙ั & $\stackrel{m}{\sigma}^{2}$ & ڤ̆ & $\vec{\sigma}$ & $\stackrel{m}{\sigma}^{-}$ & $\tilde{\sigma}$ & $\vec{\sigma}$ & $\tilde{m}^{\prime}$ & $\tilde{\sigma}$ & 으 & 음 & $\vec{\sigma}$ & $\tilde{\sigma}$ & $\tilde{m}^{\prime}$ & 으 & $\stackrel{\circ}{\circ}$ & $\sigma$ & $\sigma^{2}$ & $\vec{\sigma}$ & $\infty$ & & $\begin{array}{l}\sigma \\
\infty\end{array}$ \\
\hline$S \times 100$ & -1 & & $\tilde{o}_{0}^{n}$ & $\stackrel{\infty}{\circ}$ & $\neg$ & g̊ & $\sim$ & $\neg$ & $0^{\circ}$ & 0 & $\sigma^{\circ}$ & $\stackrel{\infty}{0^{-}}$ & $\stackrel{N}{\sim}$ & $\stackrel{\text { Ln }}{\rightarrow}$ & $\stackrel{+}{\rightarrow}$ & ğ & $\stackrel{\infty}{\circ}$ & : & $\stackrel{m}{\rightarrow}$ & $\stackrel{m}{\rightarrow}$ & 눙 & $\stackrel{6}{r}$ & $\stackrel{\infty}{\circ}$ & 경 & ฉ̊- & g̊ & $\vec{r}$ & $\stackrel{n}{\rightarrow}$ & $\stackrel{n}{\rightarrow}$ & $\stackrel{0}{\circ}$ & $\stackrel{\circ}{\rightarrow}$ & 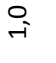 & $\stackrel{\circ}{\rightarrow}$ & $\stackrel{n}{\circ}$ & g్ & 응 & $\stackrel{0}{0}$ & $\stackrel{m}{\rightarrow}$ & $\stackrel{m}{o}$ & 응 & 가 & $\sim$ & -1 & శ̊- & o & & o \\
\hline I & & 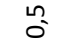 & $\stackrel{0}{0}$ & 0 & 0 & ํㅜㅇ & 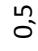 & $0^{\prime}$ & & 0 & & นn & ํㅜㅇ & ํㅜㅇ & Ln & นn & ํㅜㅇ & $\stackrel{6}{0}$ & นn & นn & $\stackrel{6}{0}$ & ํㅜㅇ & นn & ํㅜㅇ & ํㅜㅇ & $\tilde{\text { Ln }}$ & 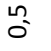 & 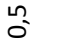 & 吕 & $\ddot{0}_{0}^{0}$ & $\stackrel{n}{o}$ & h & ñ & $\ddot{0}_{0}^{0}$ & 吕 & 吕 & ñ & $\stackrel{n}{o}$ & $\hat{o}$ & $\stackrel{n}{o}$ & $\stackrel{n}{o}$ & 0 & ז̊ & 5 & 5 & & \\
\hline
\end{tabular}
\end{tabular}

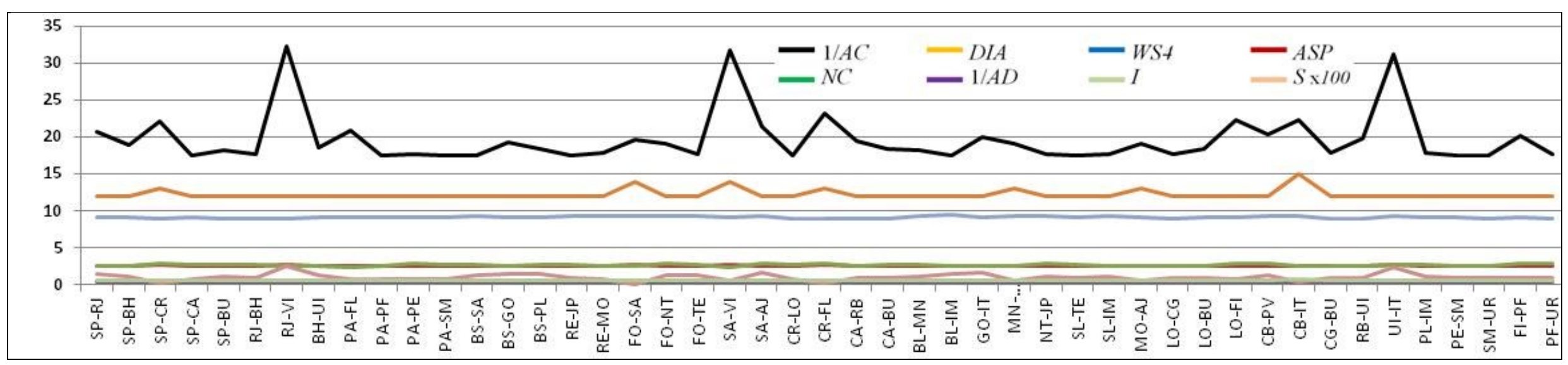

Figura B.11 - medidas após retirada de nós da rede Op_D 


\section{B.12 - Retirada de enlaces da rede Op_E}

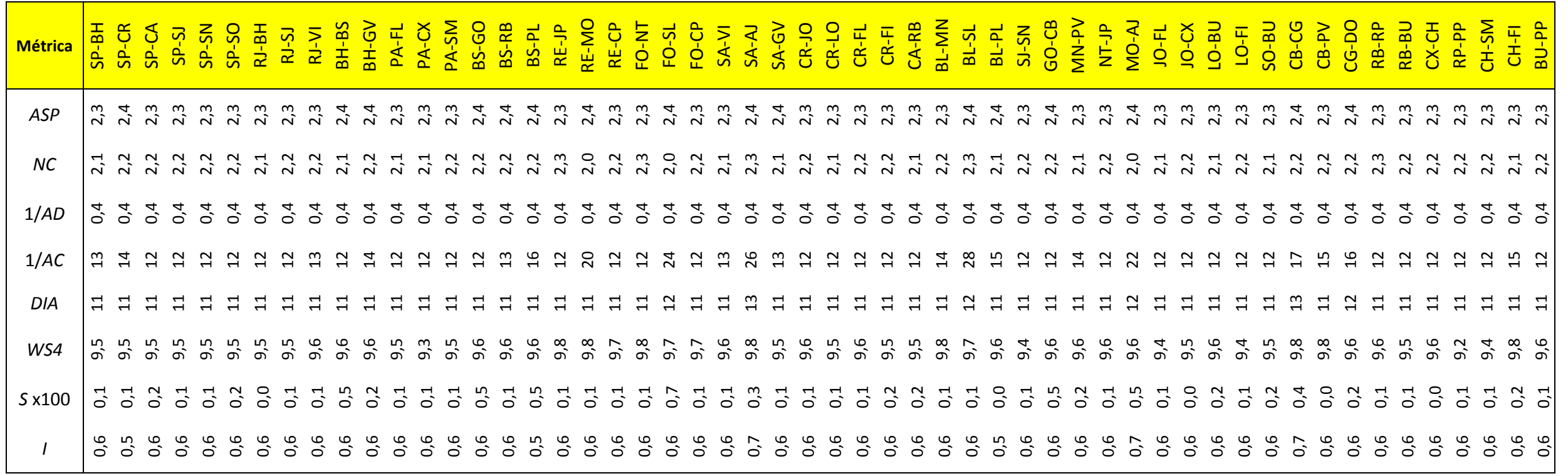

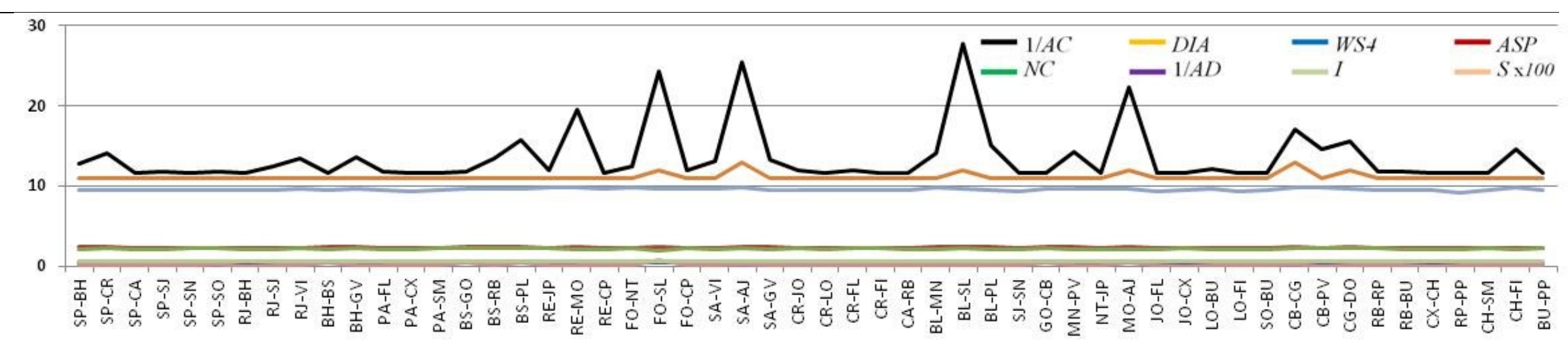

Figura B.12 - medidas após retirada de nós da rede Op_E 


\section{B.13 - Retirada de enlaces da rede Cost266}

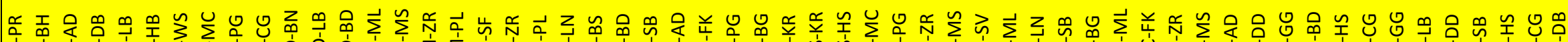

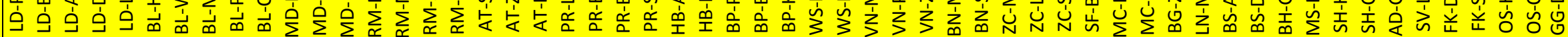

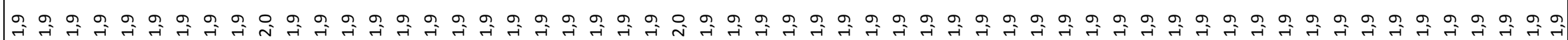

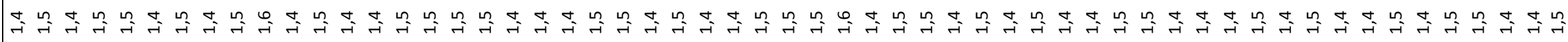

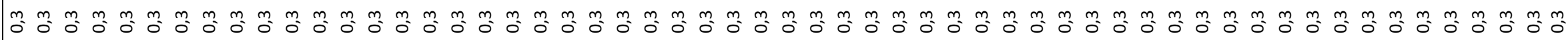

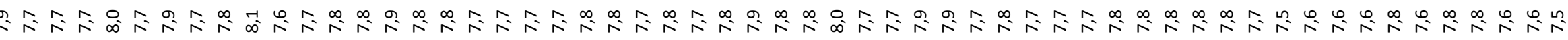

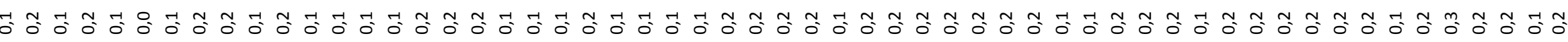

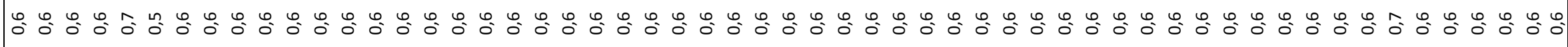

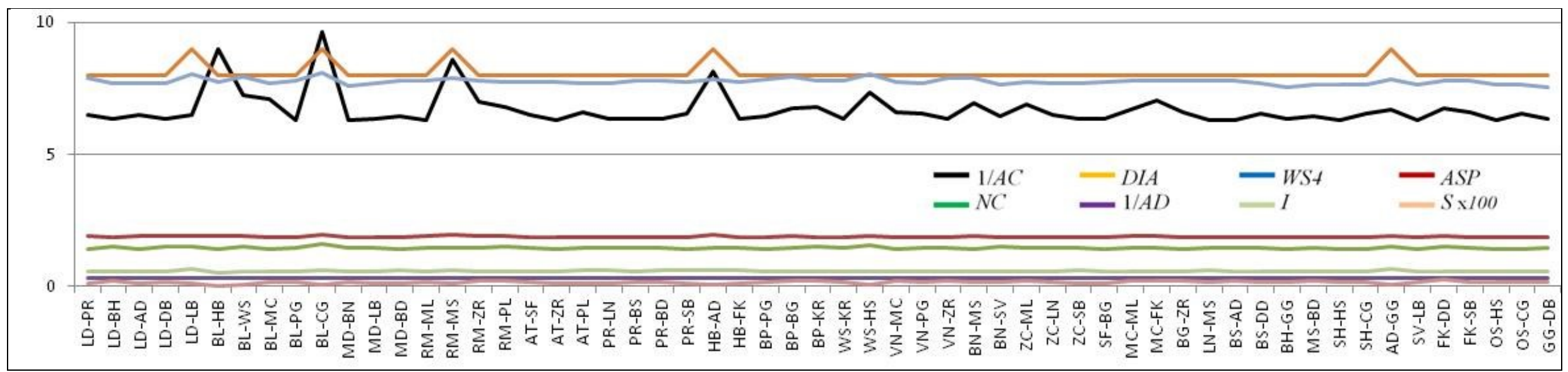

Figura B.13 - medidas após retirada de nós da rede Cost266 


\section{B.14 - Retirada de enlaces da rede Janos US-CA}

\begin{tabular}{|c|c|}
\hline Métrica & 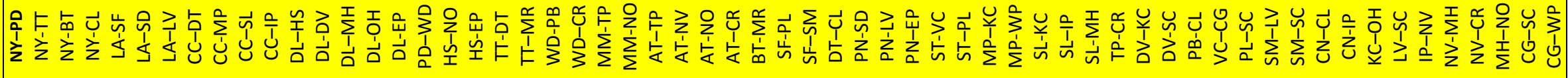 \\
\hline$A S P$ & 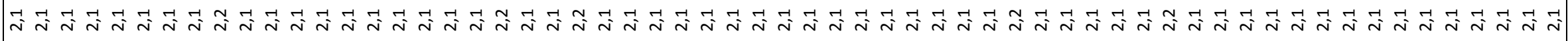 \\
\hline NC & 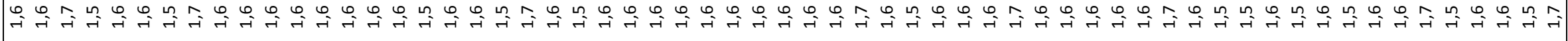 \\
\hline $1 / A D$ & 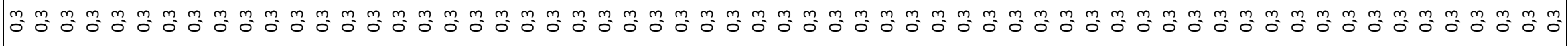 \\
\hline WS4 & 空 \\
\hline$S \times 100$ & 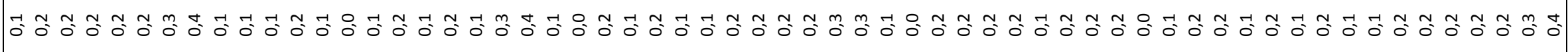 \\
\hline 1 & 00000 \\
\hline
\end{tabular}

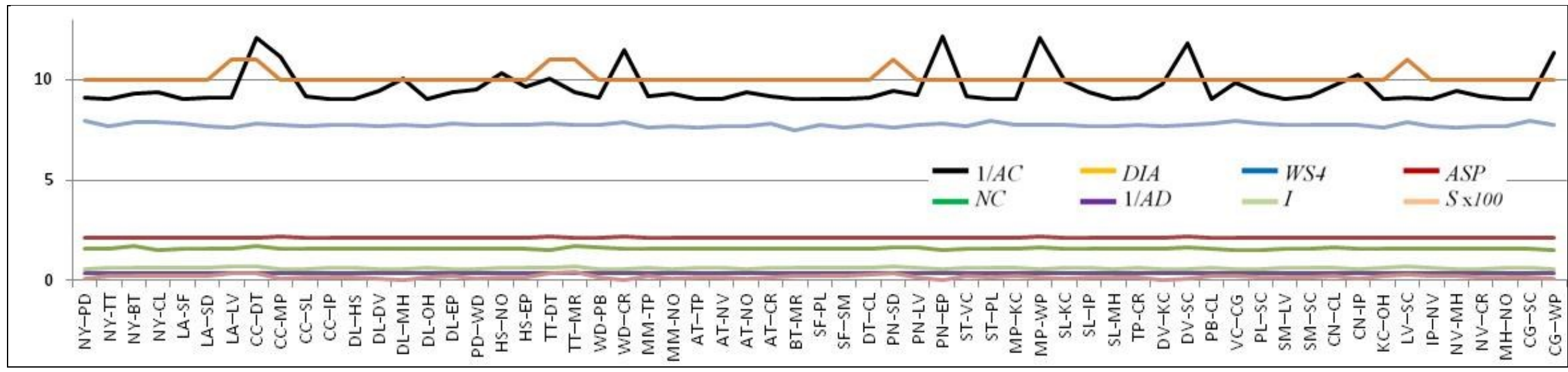

Figura B.14 - medidas após retirada de enlaces da rede Janos US-CA 


\section{Apêndice C - Programas (Scripts) \\ C.1 - Script Op_A}

응 Sig da rede = nome do arquivo Excel a utilizar

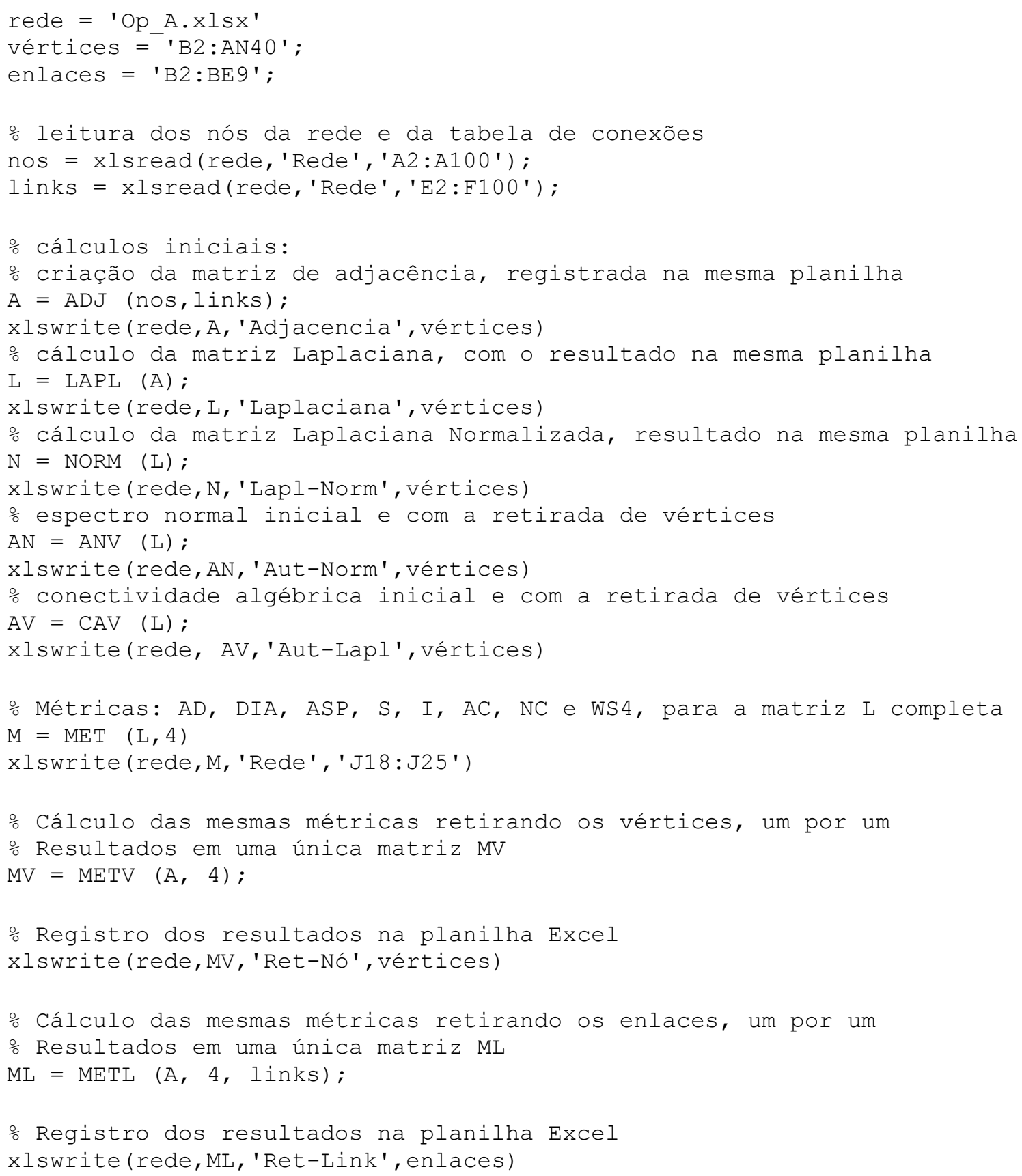




\section{C.2 - Script Op_B}

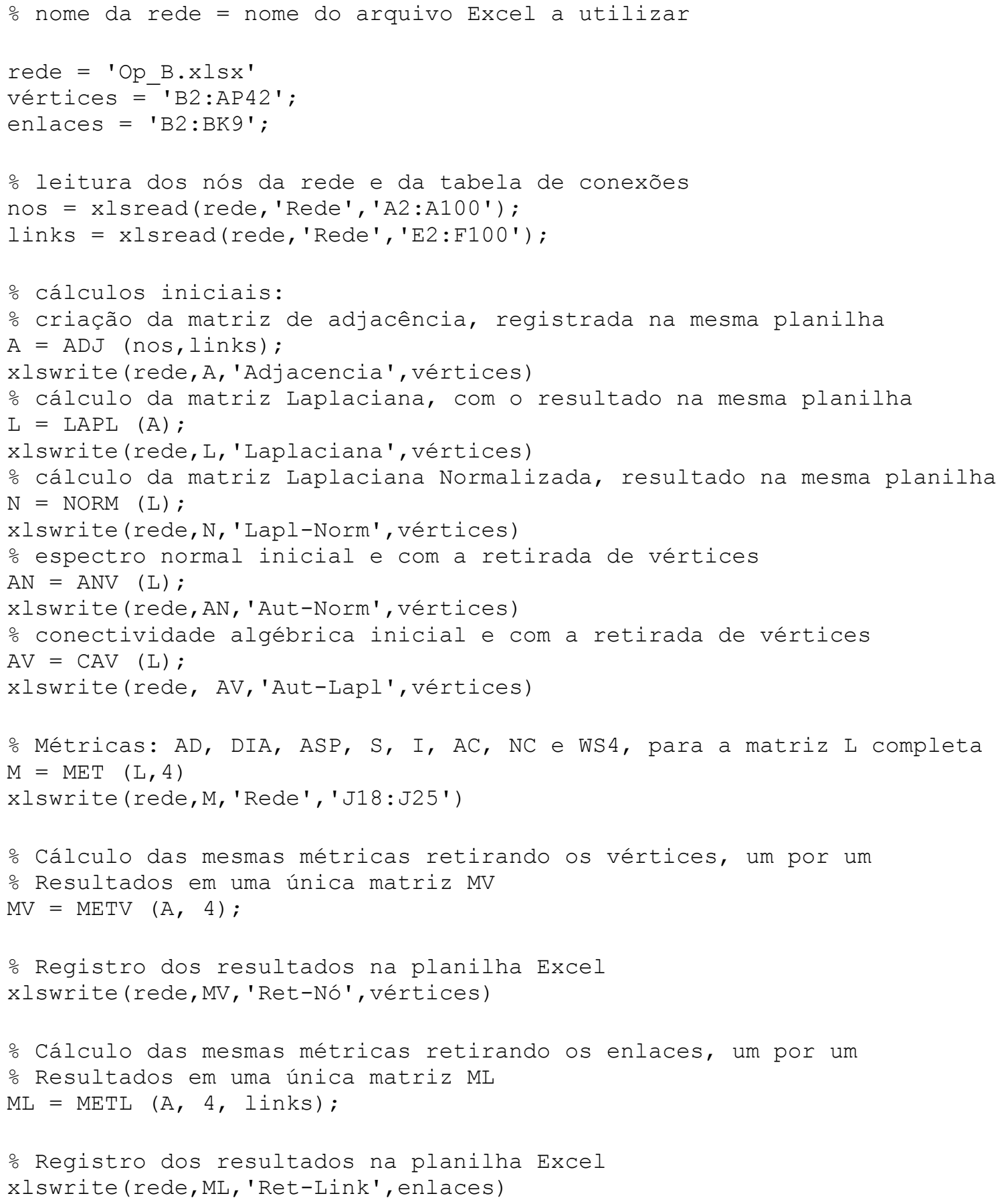




\section{C.3 - Script Op_C}

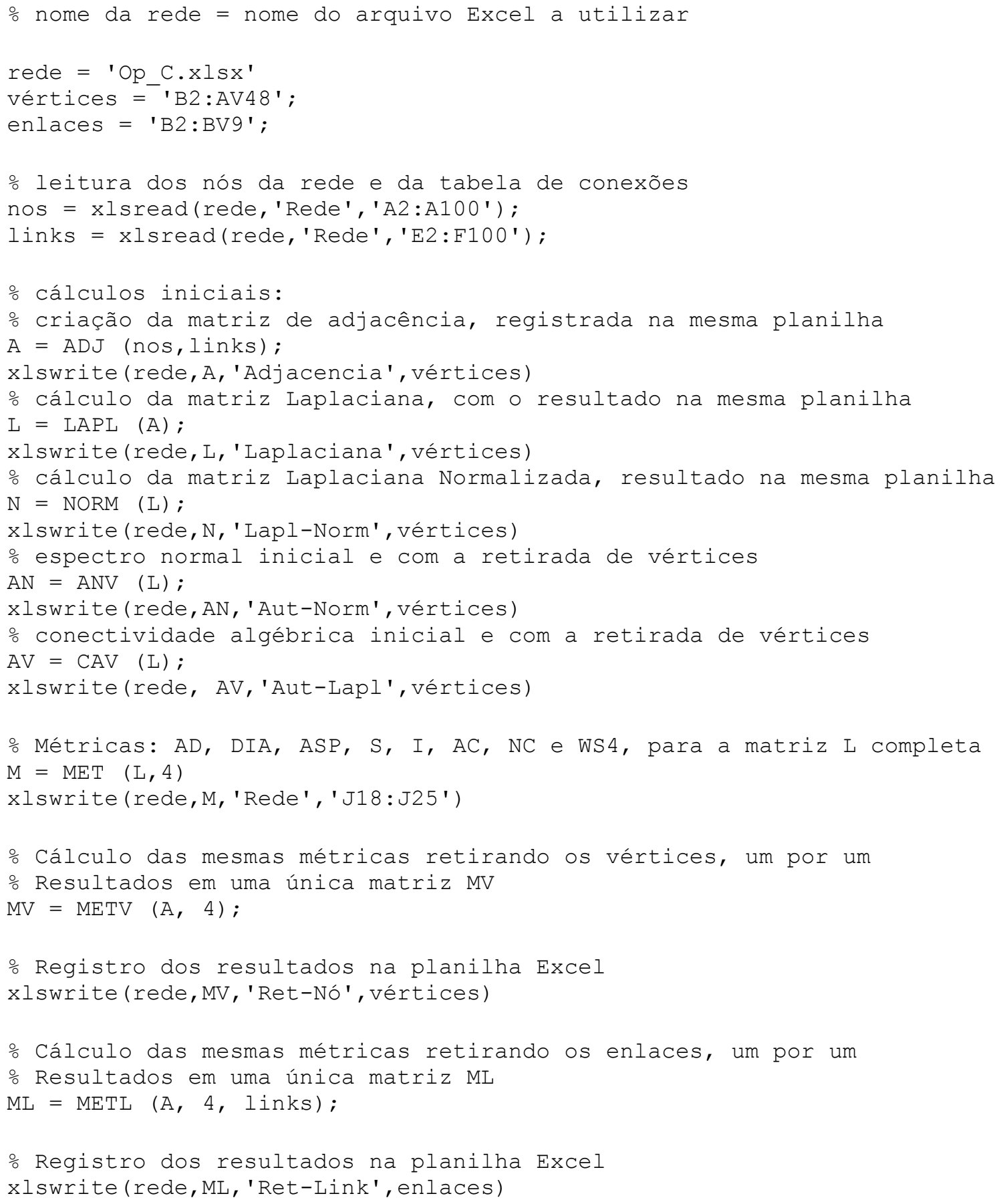




\section{C.4 - Script Op_D}

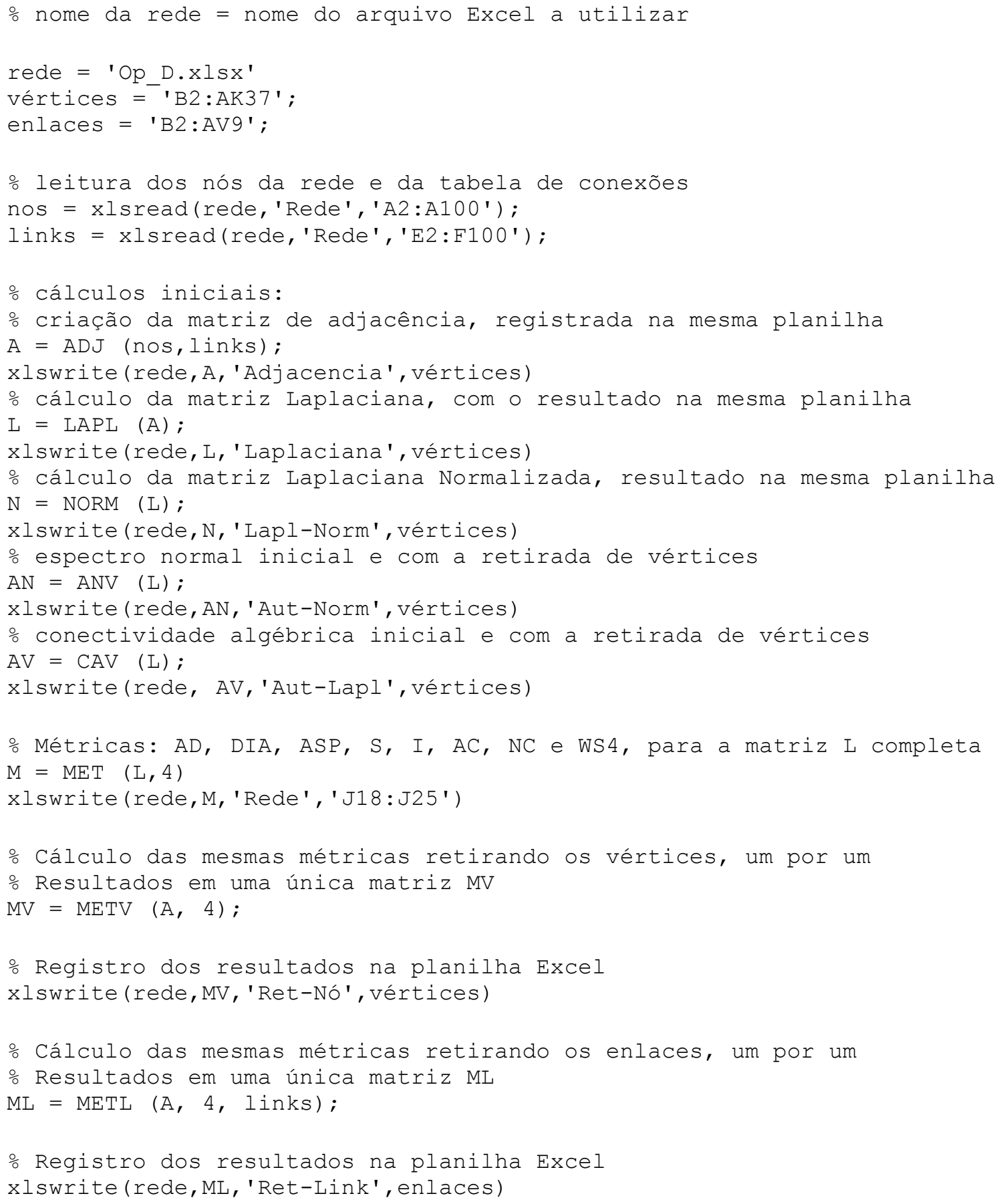




\section{C.5 - Script Op_E}

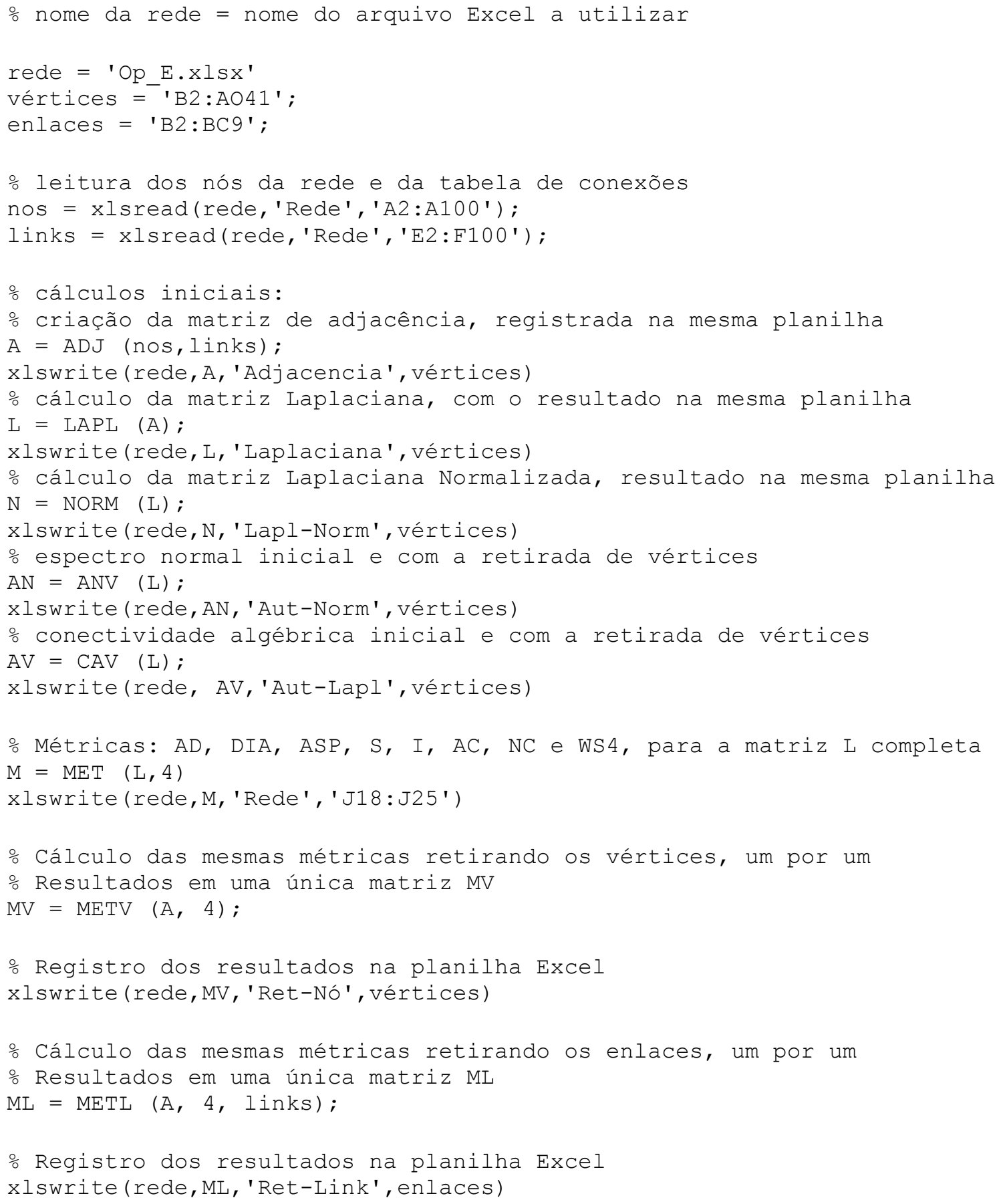




\section{C.6 - Script Cost266}

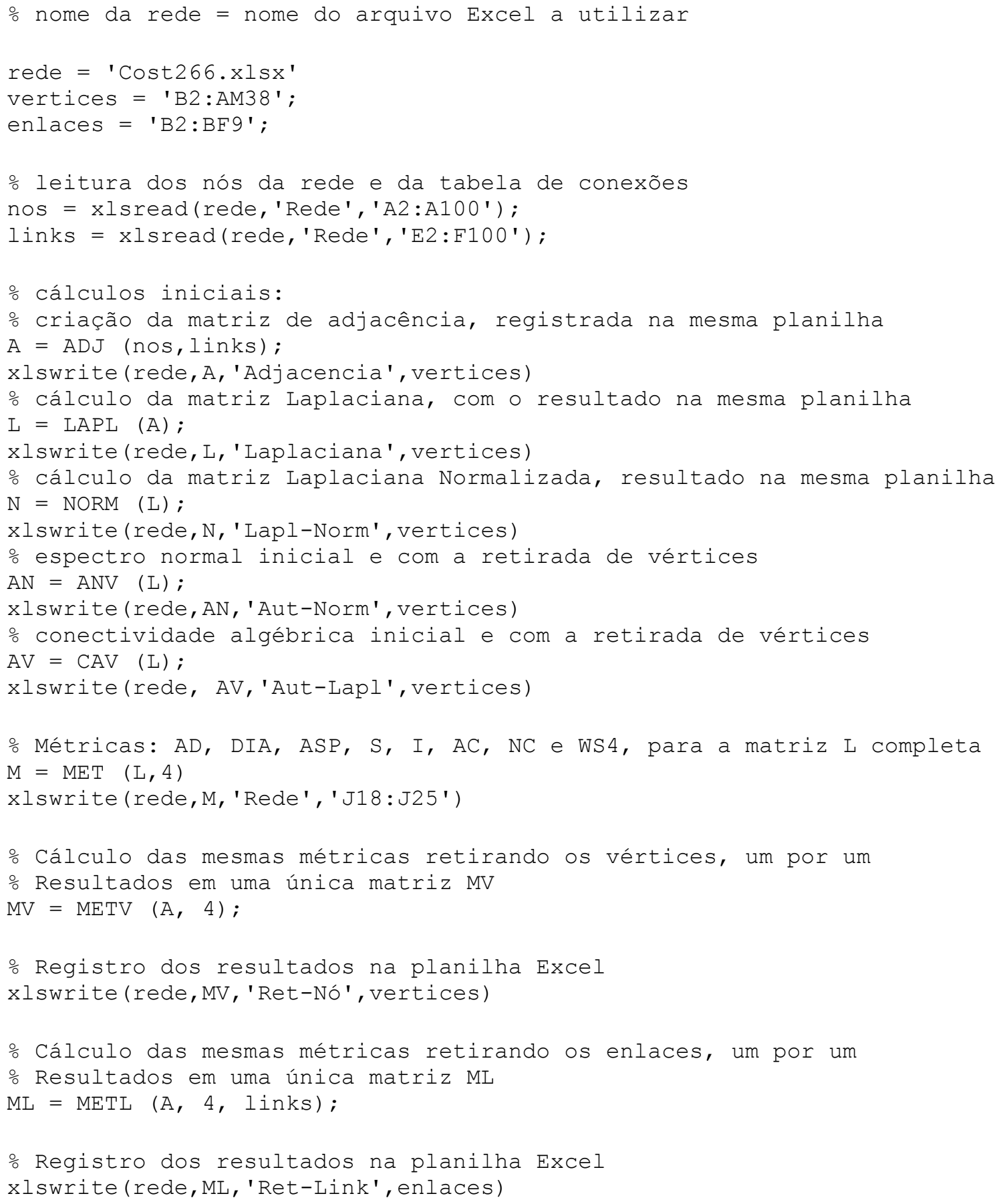




\section{C.7 - Script Janos}

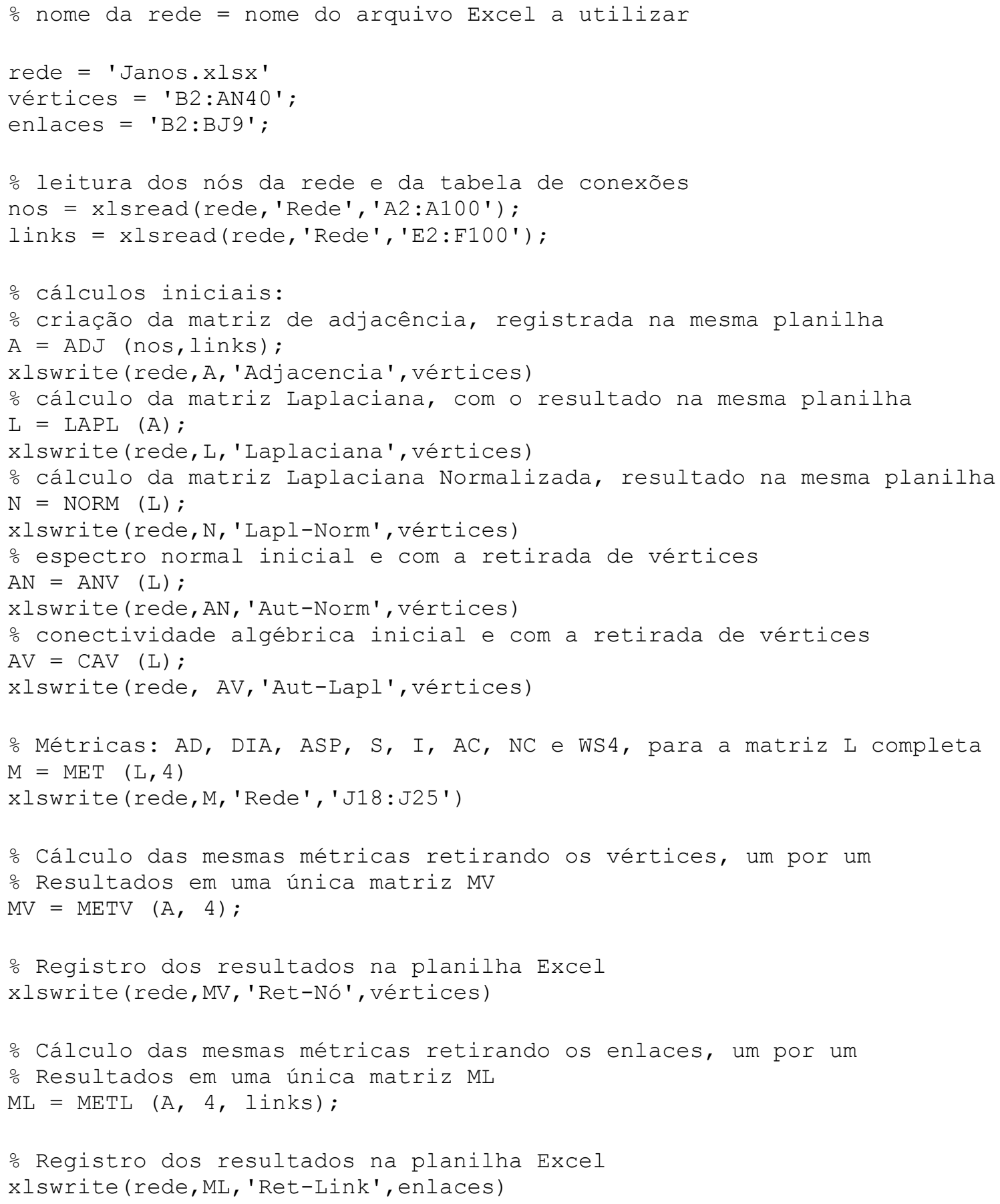




\section{Apêndice D - Funções}

\section{D.1 - Função ADJ}

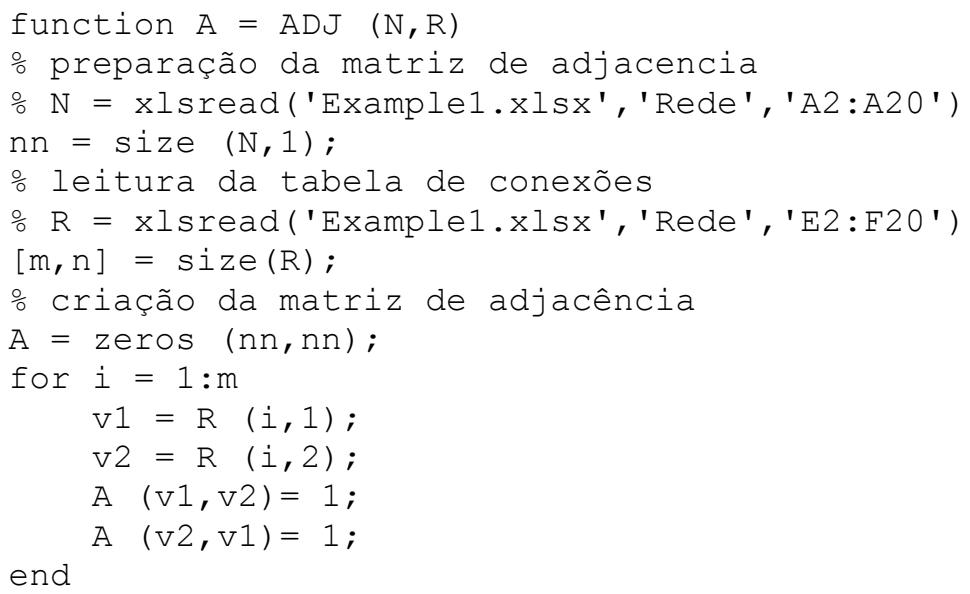

\section{D.2 - Função LAPL}

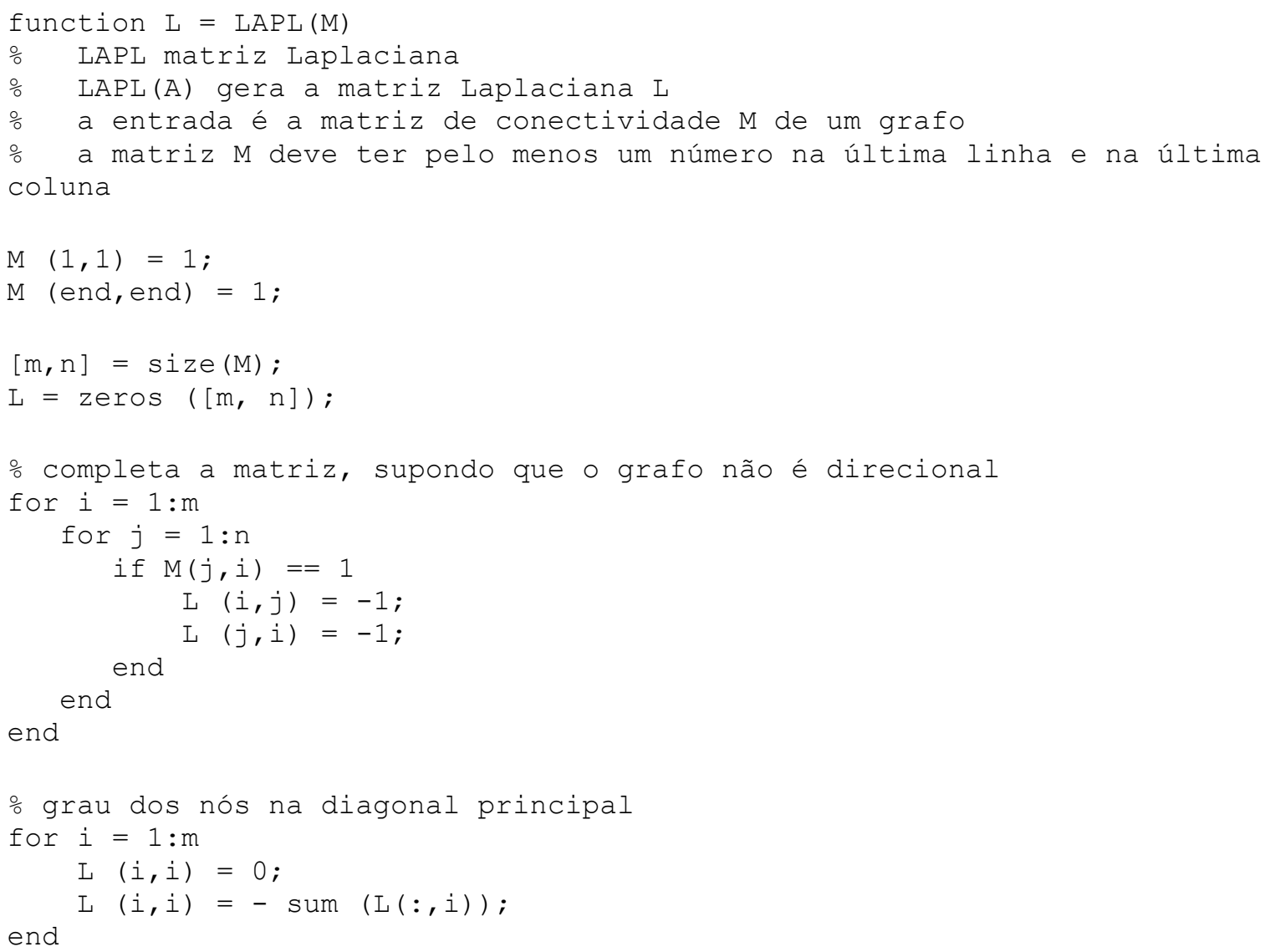




\section{D.3 - Função NORM}

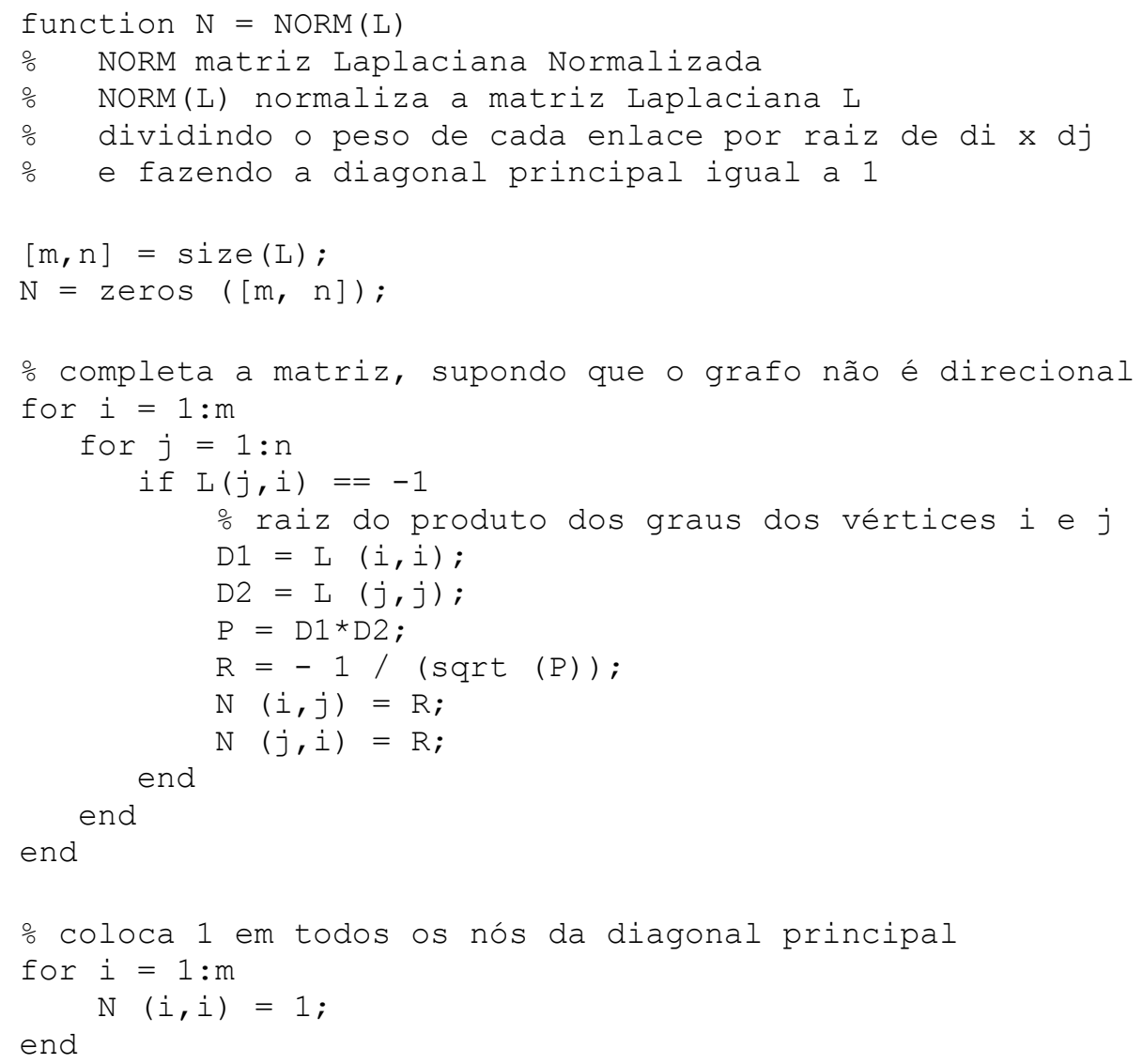

\section{D.4 - Função ANV}

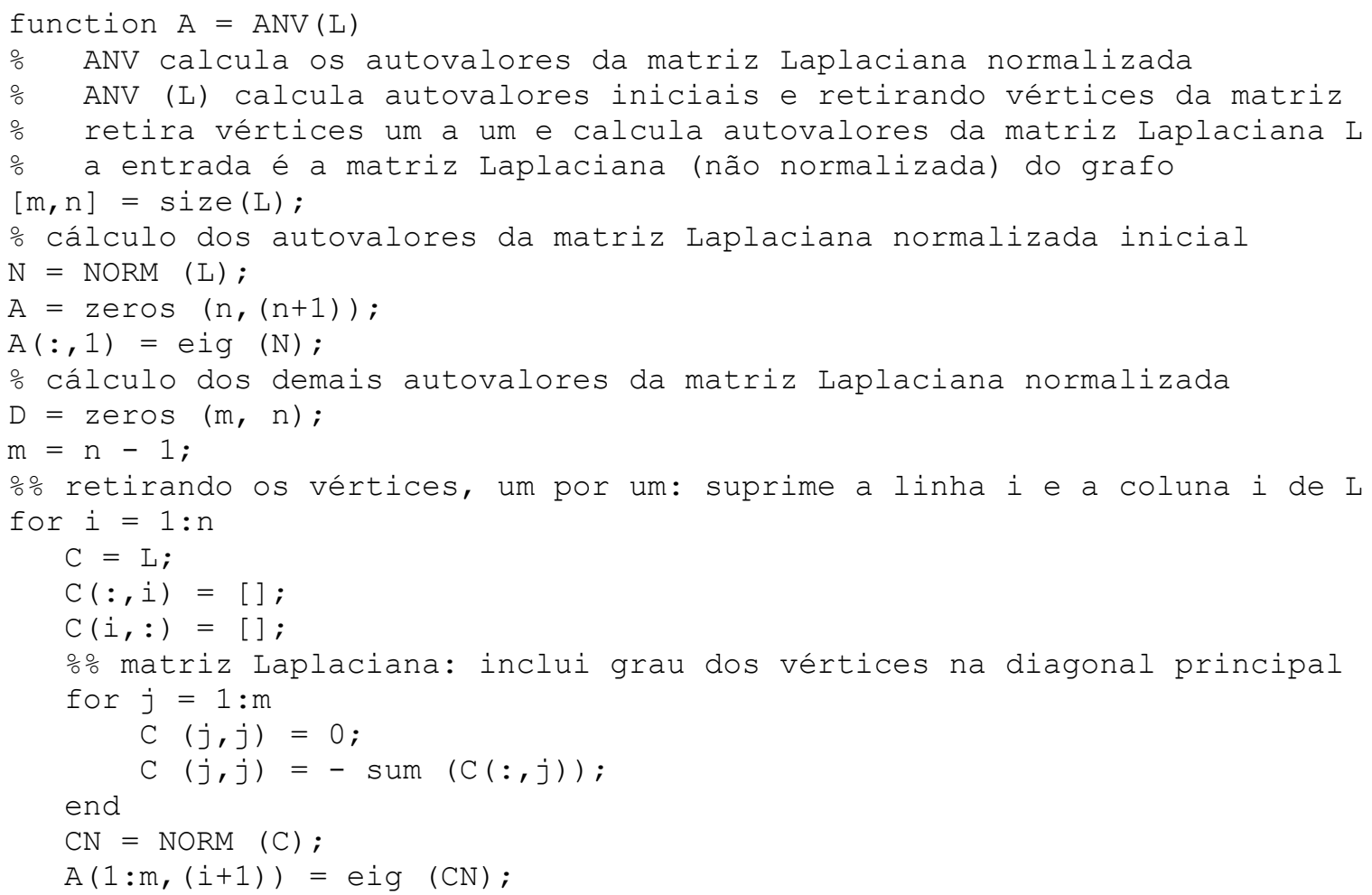




\section{D.5 - Função CAV}

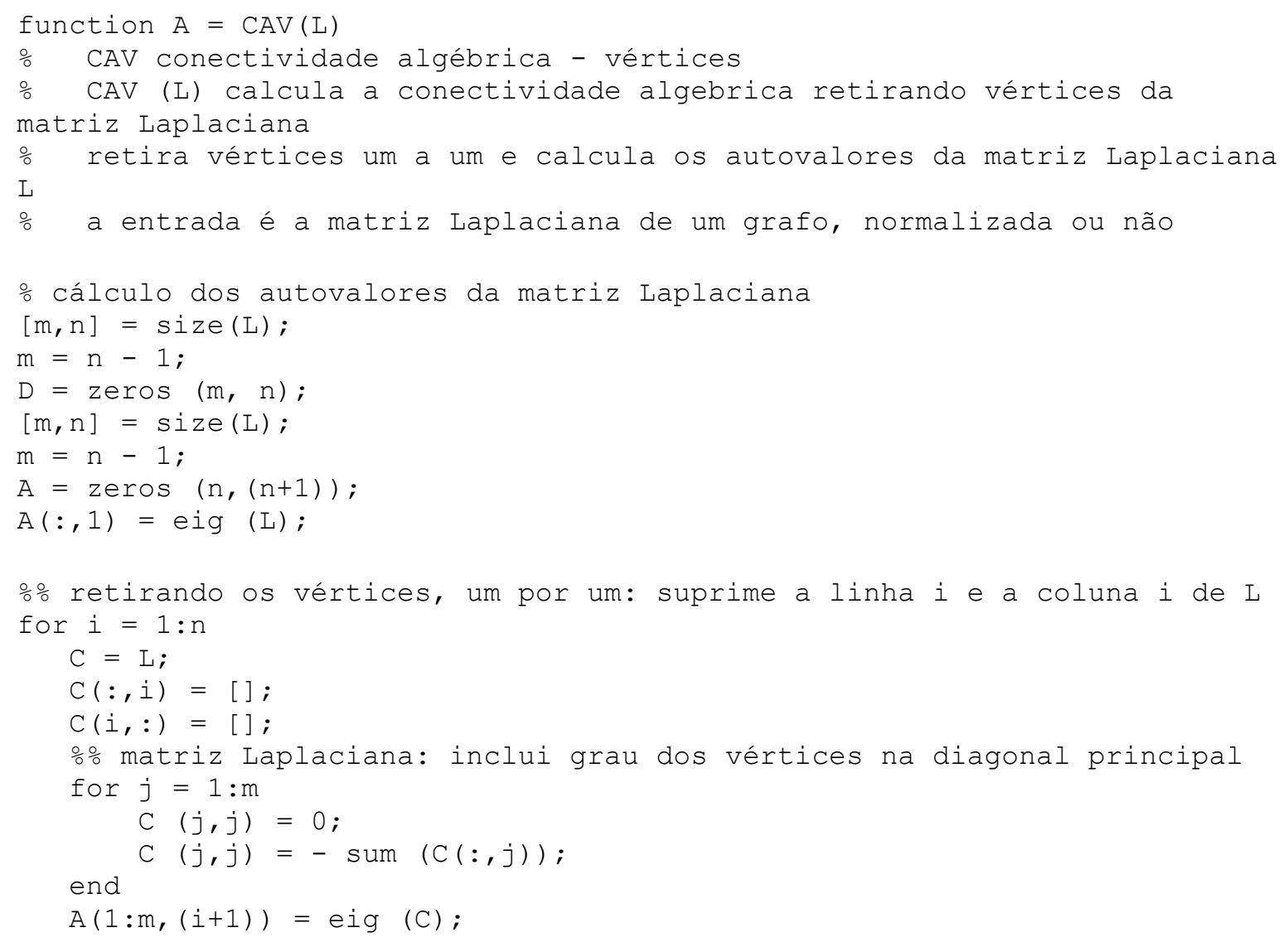




\section{D.6 - Função MET}

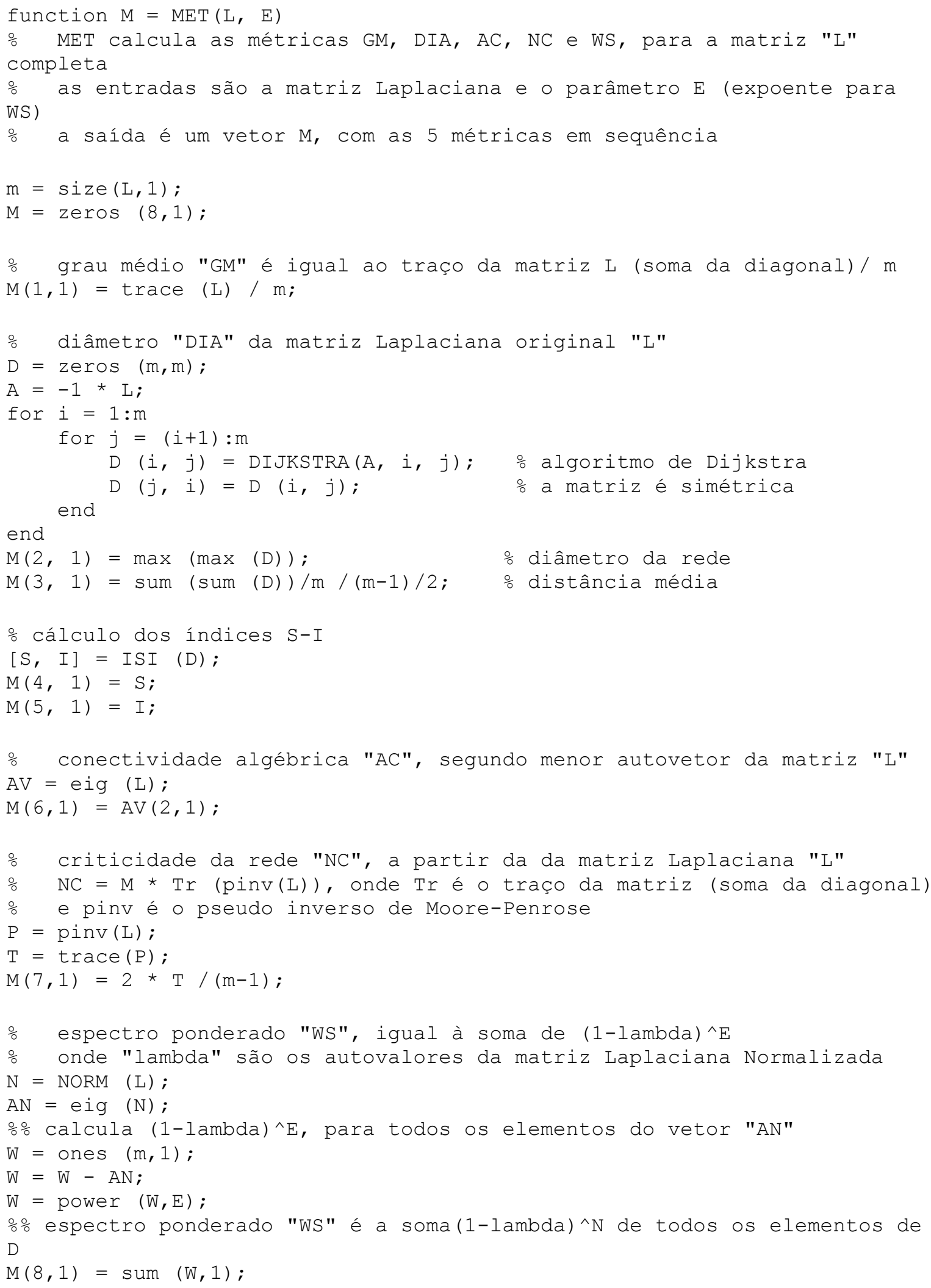




\section{D.7 - Função METV}

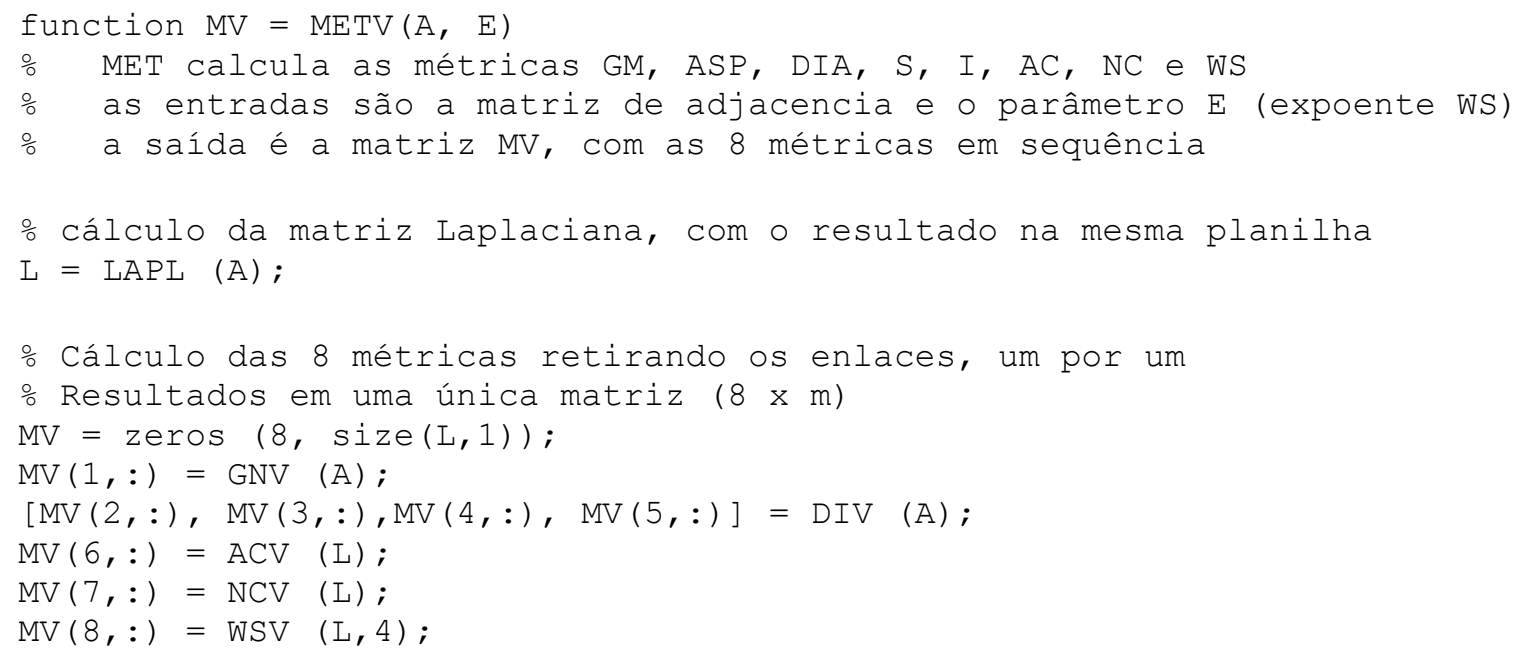

\section{D.8 - Função METL}

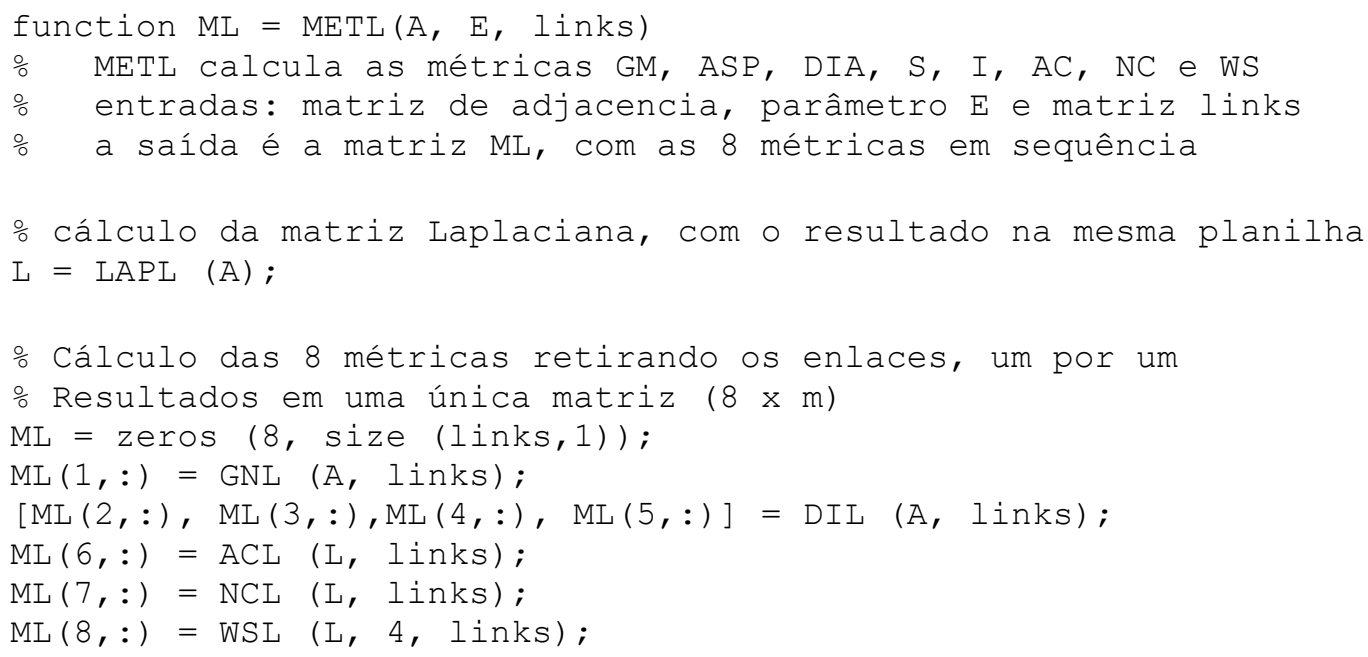




\section{D.9 - Função DJIKSTRA}

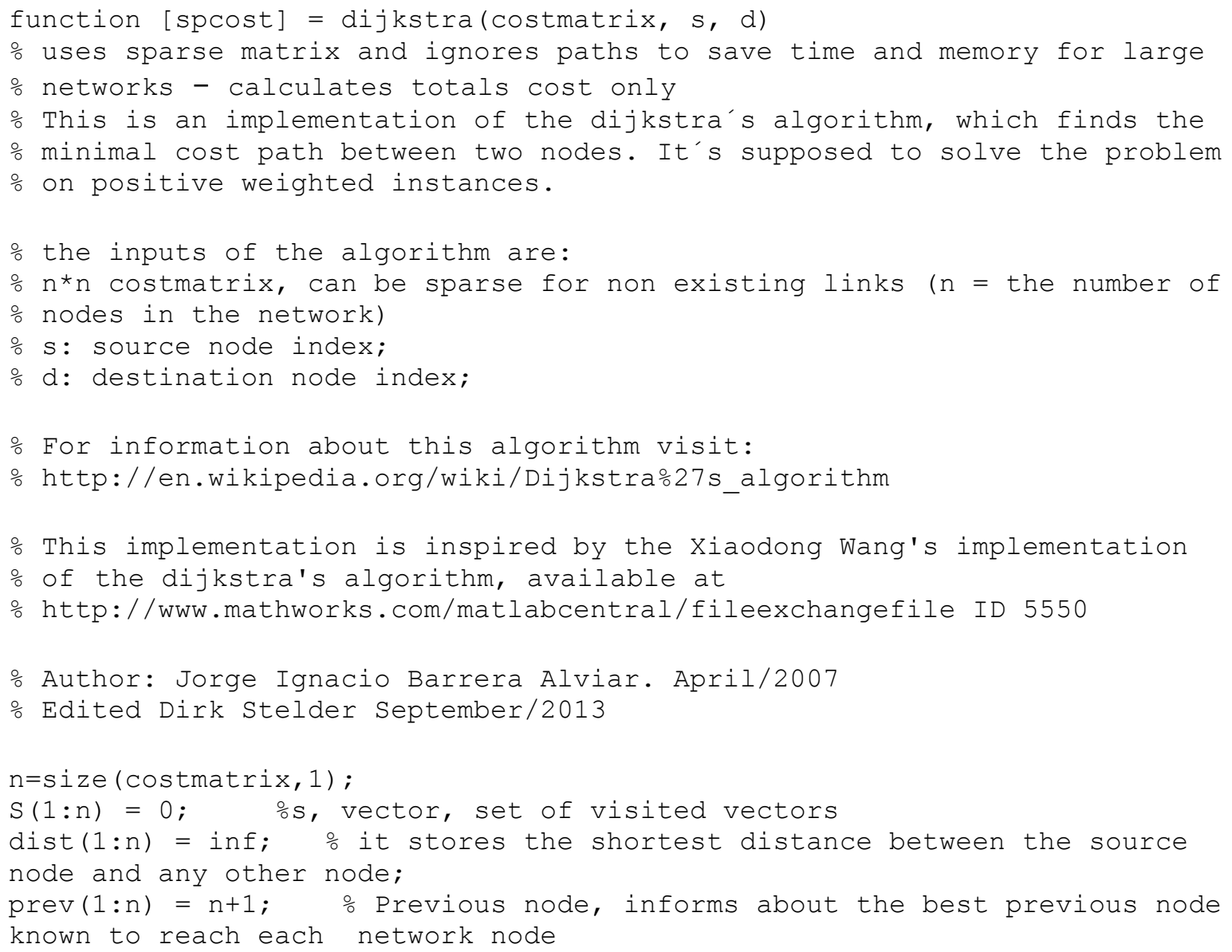




\section{D.10 - Função ISI}

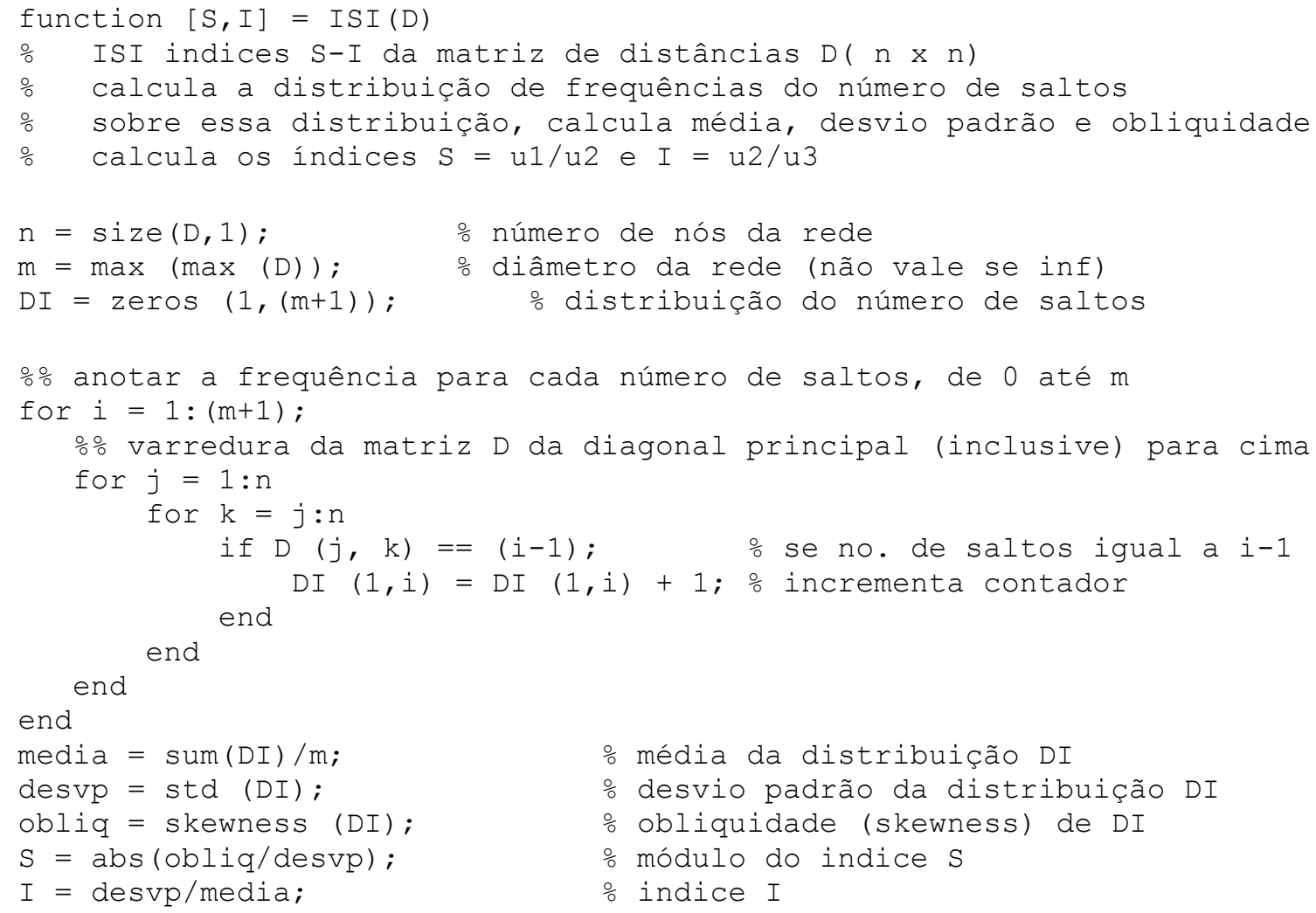

\section{D.11 - Função GNV}

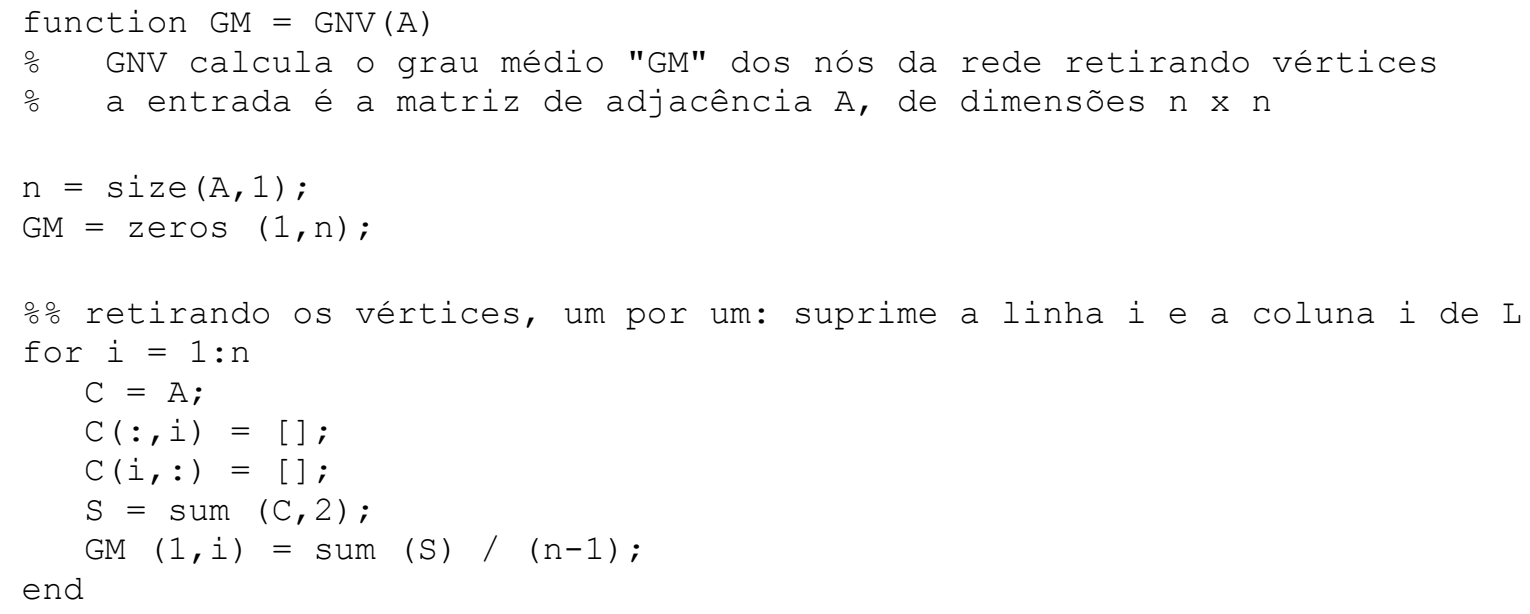




\section{D.12- Função ACV}

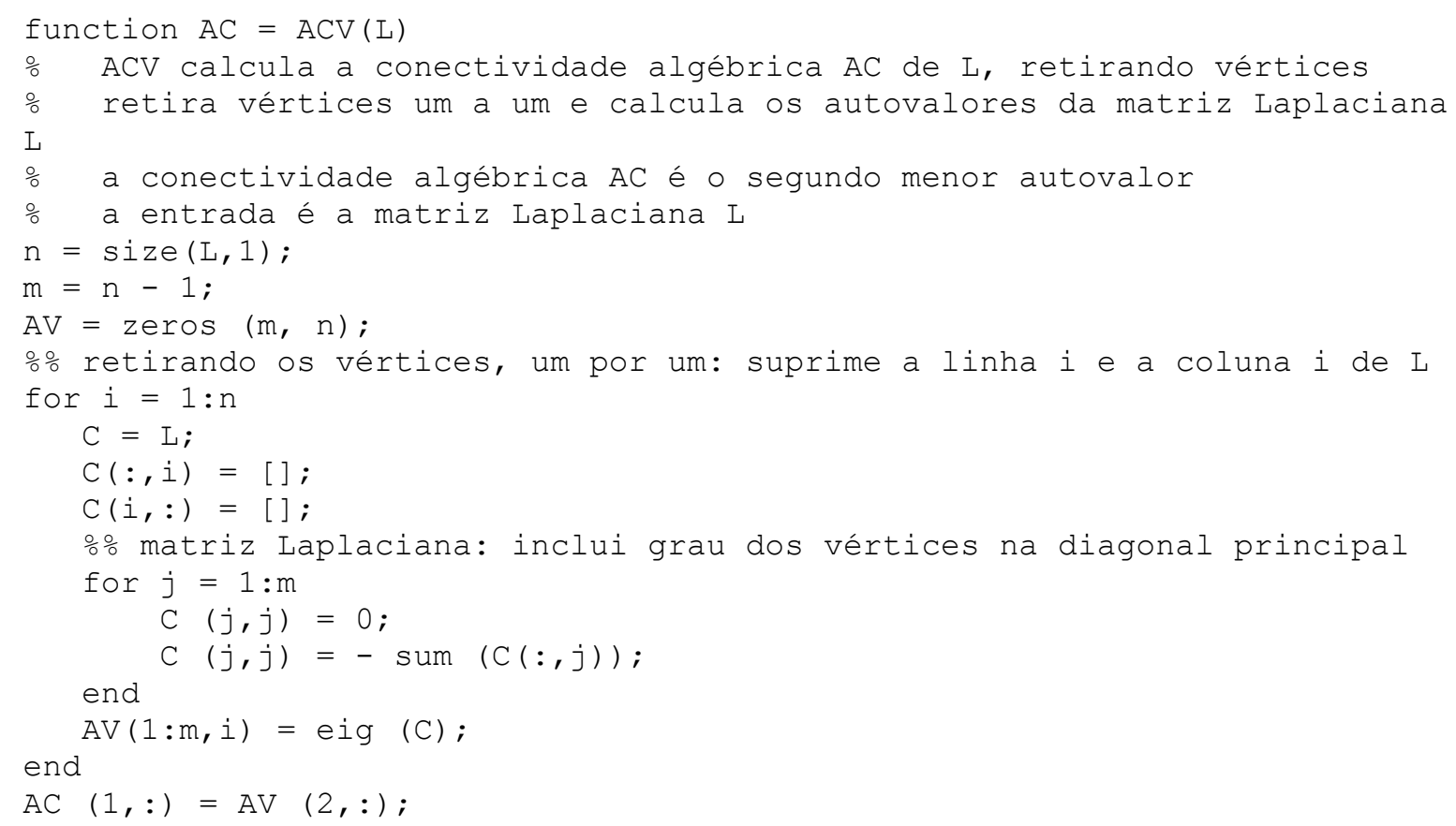

\section{D.13 - Função NCV}

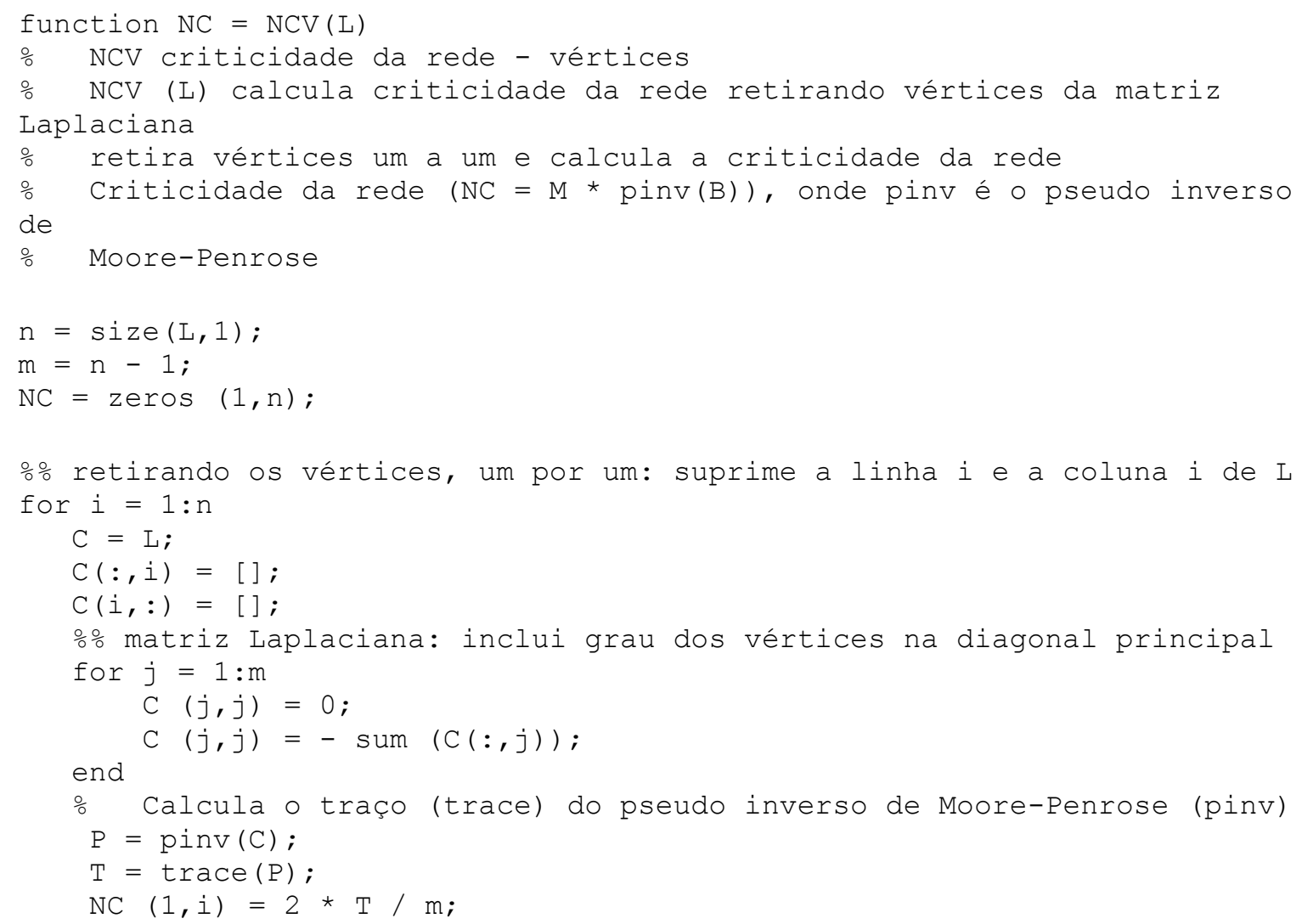




\section{D.14 - Função WSL}

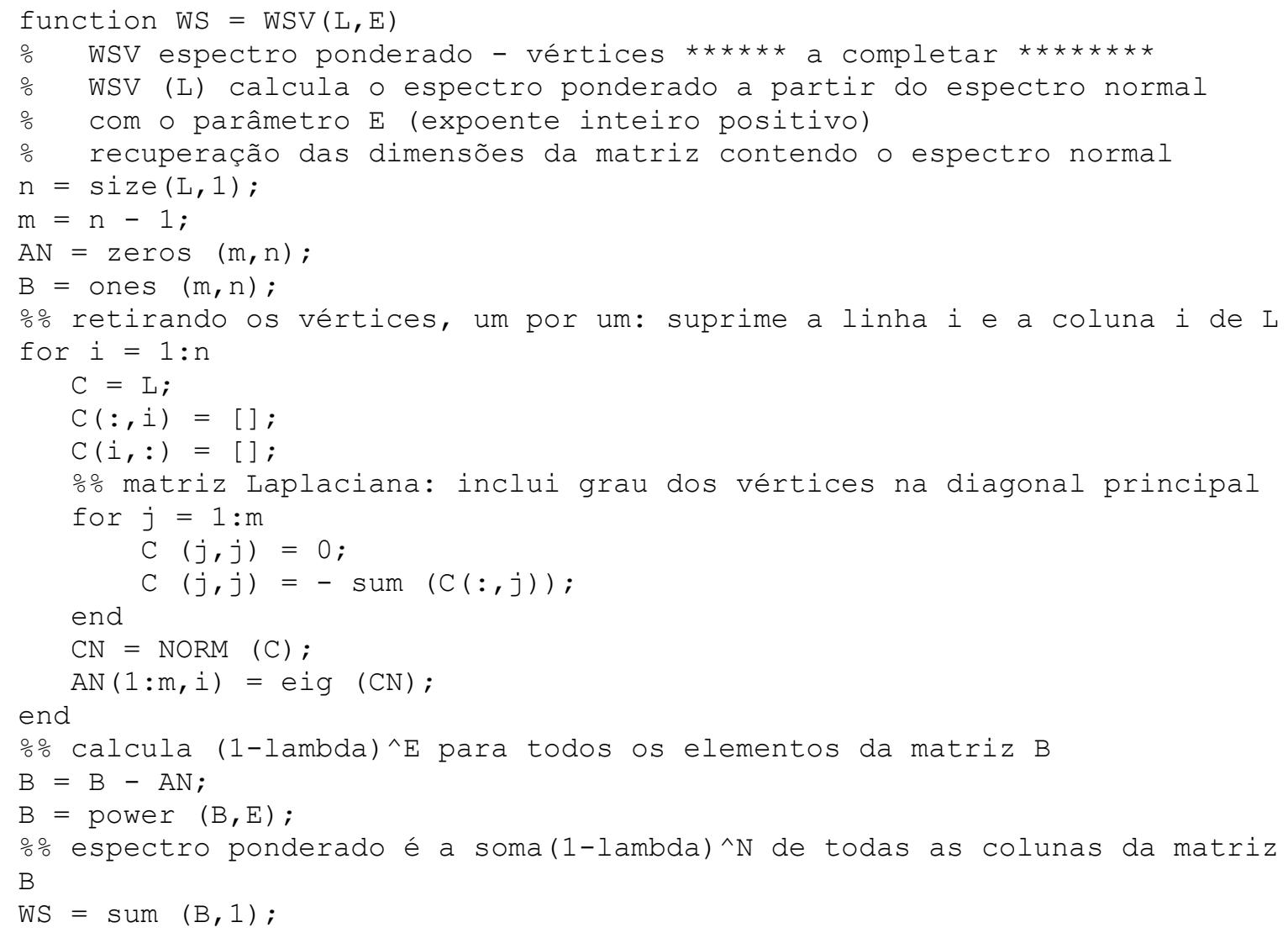

\section{D.15 - Função GNV}

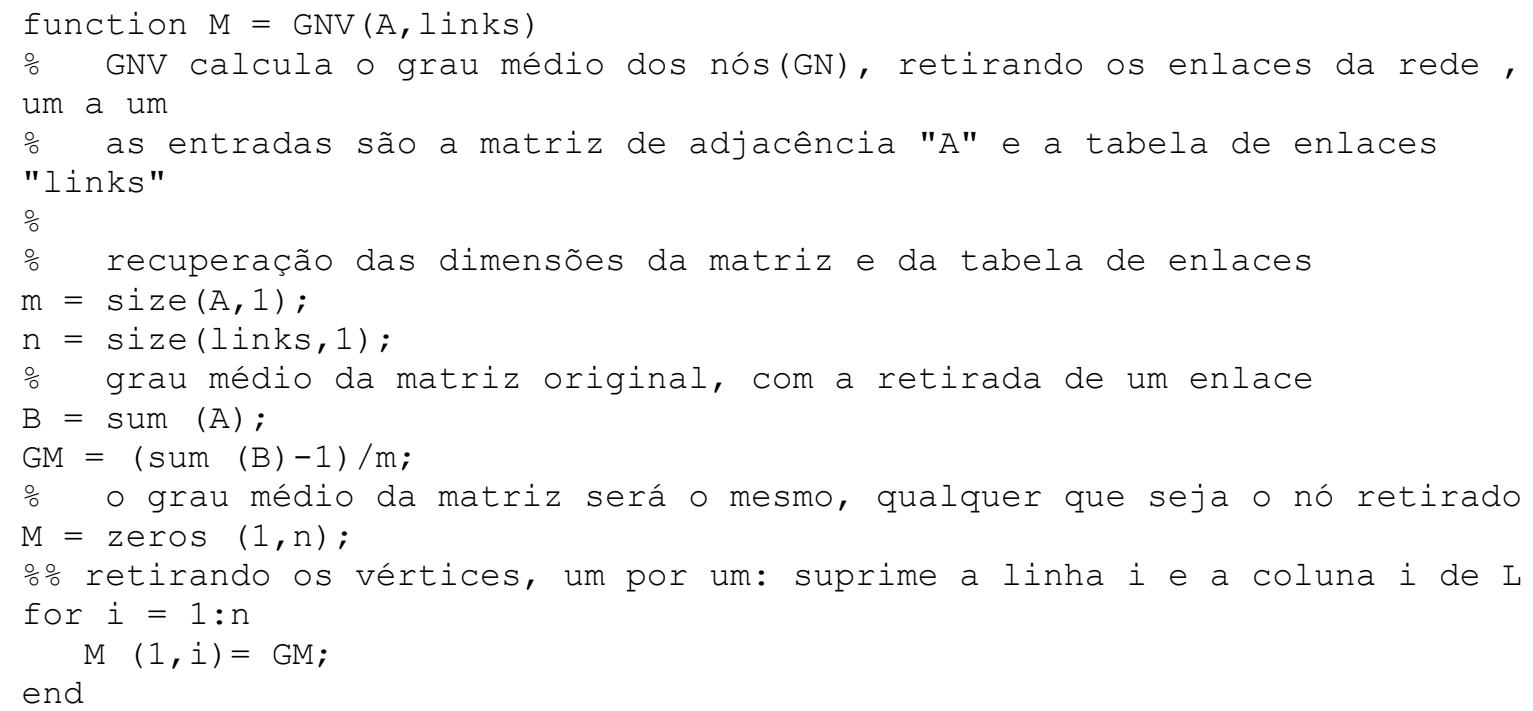




\section{D.16 - Função ACL}

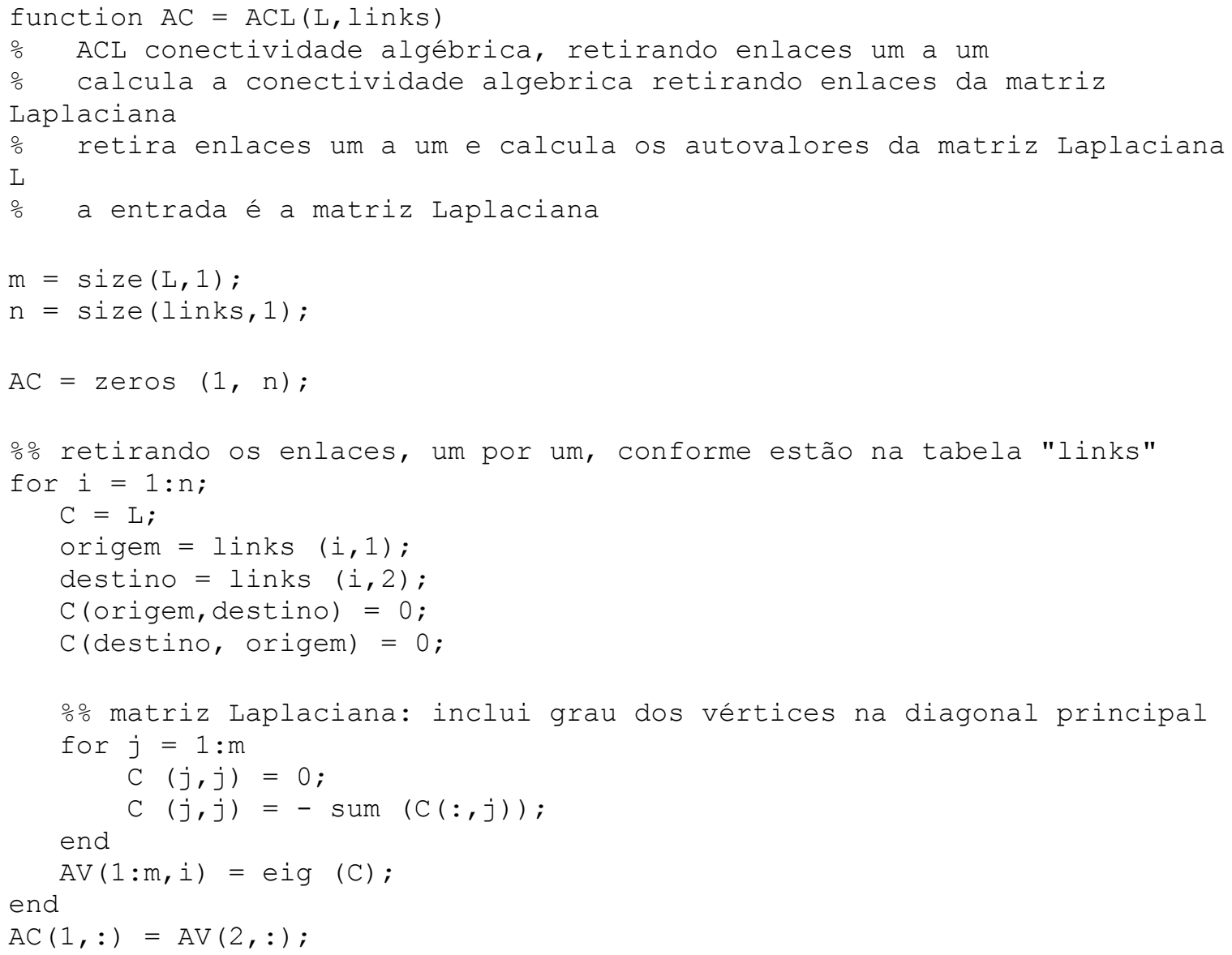




\section{D.17 - Função NCL}

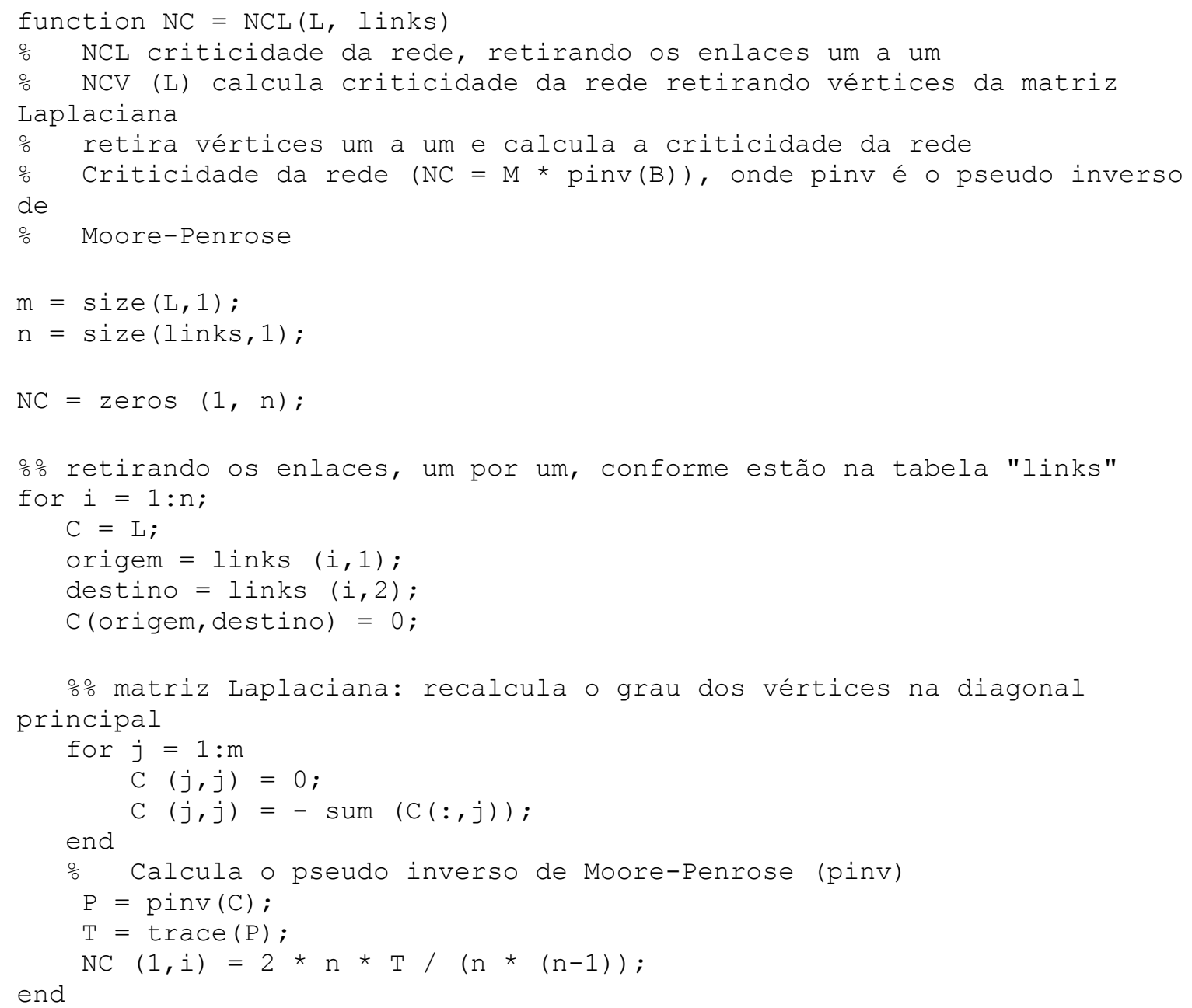




\section{D.18 - Função WSL}

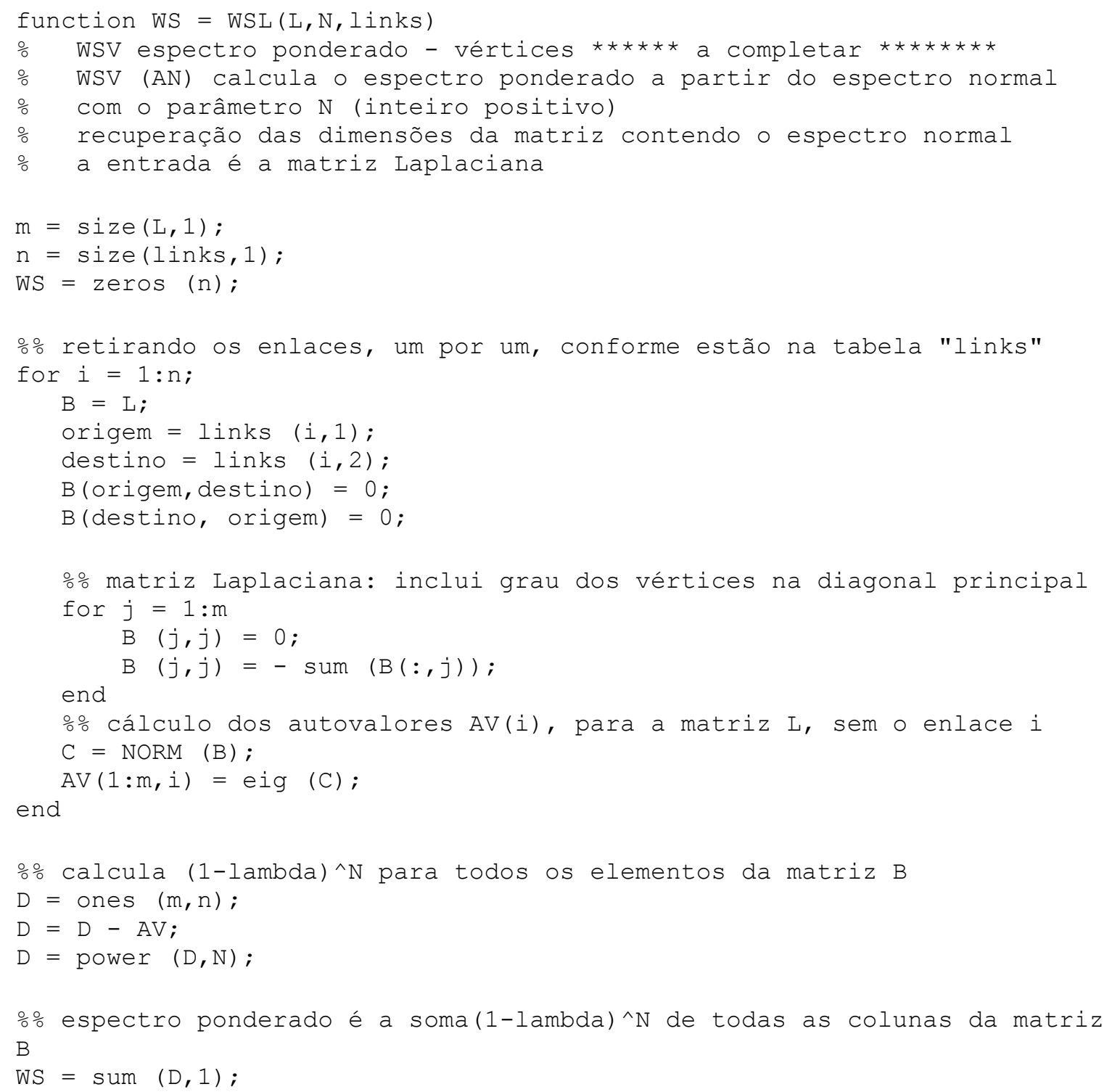


Apêndice E - Estrutura das planilhas utilizadas

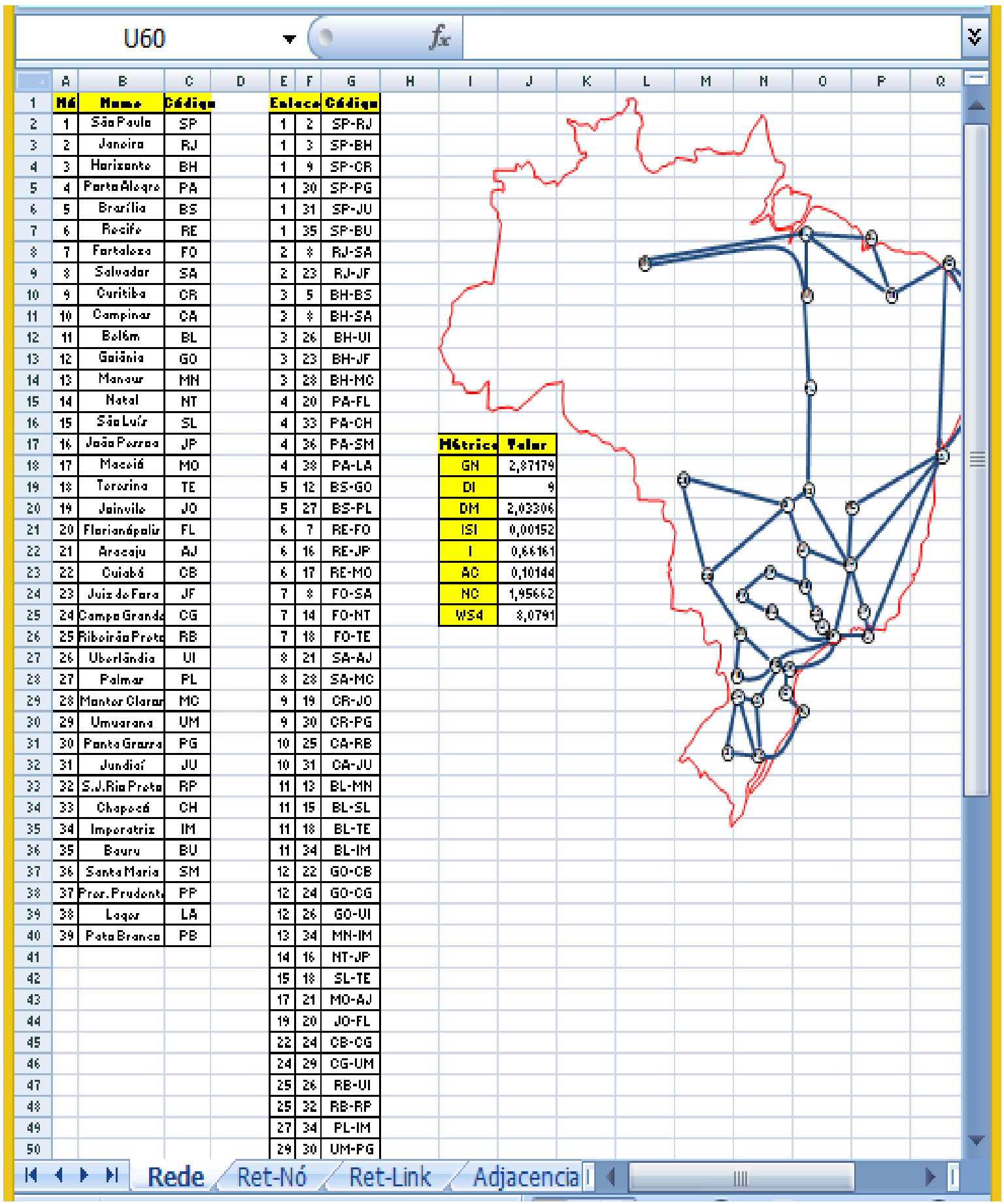

